\title{
A LATTICE-BASED MRF MODEL FOR DYNAMIC NEAR-REGULAR TEXTURE TRACKING AND MANIPULATION
}

\author{
Wen-Chieh Lin
}

CMU-RI-TR-05-58

\author{
Robotics Institute \\ Carnegie Mellon University \\ Pittsburgh, PA 15213
}

December 2005

\author{
Submitted in partial fulfilment of \\ the requirements for the degree of \\ Doctor of Philosophy

\section{Thesis Committee: \\ Yanxi Liu, Chair \\ Alexei A. Efros} \\ Robert T. Collins, Pennsylvania State University \\ Greg Turk, Georgia Institute of Technology
}




\section{ABSTRACT}

A

Near-regular texture (NRT) is a geometric and photometric deformation from its regular origin - a congruent wallpaper pattern formed by 2D translations of a single tile. A dynamic NRT is an NRT under motion. Correspondingly, the basic unit of a dynamic NRT is a well-defined texton, as a geometrically and photometrically deformed tile, moving through a 3D spatiotemporal space. Although NRTs are pervasive in man-made and natural environments, effective computational algorithms for NRTs are few. Through a systematic and quantitative comparison study of multiple texture synthesis algorithms, we are able to show that faithful NRT synthesis has challenged most of the state of the art texture synthesis algorithms. Our recent work on static NRTs analysis and manipulation [Liu et al., 2004] is the first algorithmic treatment aimed specifically to preserve the regularity and randomness in real-world near regular textures.

The theme of this thesis is to address computational issues in modeling, tracking and manipulating dynamic NRTs. One basic observation on dynamic NRT is its topology invariance property: the lattice structure of a dynamic NRT remains invariant despite its drastic geometry or appearance variations. We propose a lattice-based MarkovRandom-Field (MRF) model for dynamic NRT in a 3D spatiotemporal space. Our dynamic NRT model consists of a global lattice structure that characterizes the topological constraint among multiple textons and an image observation model that handles local geometry and appearance variations. Our model behaves like a network of statistically varied springs. Based on our dynamic NRT model, we develop a tracking algorithm that can effectively handle the special challenges of dynamic NRT tracking, including: ambiguous correspondences, occlusions, illumination variations, and appearance variations. Our algorithm does not assume the type of motion that a dynamic NRT may undergo. Furthermore, we implement a dynamic NRT manipulation system that can replace and superimpose images on a dynamic NRT from an unknown environment.

The main contributions of this thesis are:

First, a novel and general quadrilateral lattice-based MRF model is proposed for dynamic NRT.

Second, we implement a dynamic NRT tracking algorithm that can effectively handle real-world dynamic NRT with occlusions.

Third, the proposed dynamic NRT framework makes it possible to accomplish several video editing and manipulation tasks, including real-world dynamic NRT synthesis, replacement, and superimposition. 



\section{ACKNOWLEDGEMENTS}

I would like to thank my advisor Yanxi Liu for her guidance and inspiration throughout these years. She taught me the value of facing challenges with perseverance and optimism. I thank my thesis committee members, Professors Bob Collins, Greg Turk, and Alyosha Efros. Bob gave many insightful suggestions on my tracking approach and timely feedback on my writing and presentations. Greg provided a different perspective on my work, which really helped to improve this thesis. Alyosha always provided valuable comments and suggestions about my work. The underwater texture tracking is inspired by his earlier work at CMU.

I also thank my former advisors, Professors Jessica Hodgins and Gary Fedder for strengthening and broadening my research skills and experience; Professors Doug James, James Kuffner, and Nancy Pollard for their helpful thoughts and advice about my research along the way.

This thesis benefited from many valuable and insightful discussions with my classmates and friends at CMU. I thank my officemate Jiayong Zhang, who suggested that I use belief propagation to solve the spatial inference problem; Jing Xiao for his suggestions on image alignment techniques; Sanjiv Kumar for his comments and helpful discussions on Markov random fields.

I was very lucky to have many great colleagues in my research projects. Yanghai Tsin provided his texture replacement code to me in the early stage of my thesis work. I thank James Hays for his help on our SIGGRAPH paper and proofreading several of my papers. Chenyu Wu implemented an initial version of deformable texture synthesis algorithm and a nice GUI for interactive lattice extraction.

I thank my friends, Liu Ren, Kiran Bhat, Wei-Tech Ang, Jinxiang Chai, Chris Twigg, Scott Jones, Alla Safonova and Paul Reitsma for accompanying me these years. Because of them, I had a wonderful and enjoyable journey at CMU.

Special thanks to my classmate and close friend, Chieh-Chih (Bob) Wang, who kept encouraging me and giving me critical advice during my study.

I thank Janice Brochetti and Suzanne Lyons Muth for their administrative support. Janice also helped to proofread this thesis.

Finally, I want to thank my parents for their endless love and continuing support in my life. They not only allow me great freedom to pursue my dreams but also encourage me to never lose faith in my ability to fulfill my dreams in the face of difficulty. 



\section{TABLE OF CONTENTS}

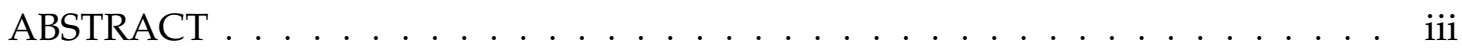

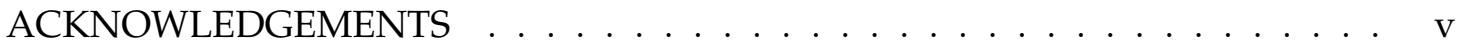

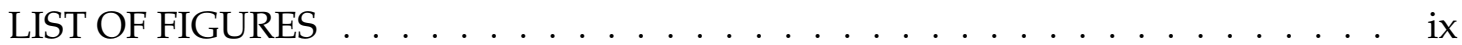

LIST OF TABLES . . . . . . . . . . . . . . . . . . . . . . . . . xvii

CHAPTER 1. Introduction . . . . . . . . . . . . . . . 1

1.1. What is a near-regular texture? . . . . . . . . . . 3

1.2. Static NRT Analysis and Manipulation . . . . . . . . . . . . . 5

1.3. Dynamic NRT Modeling, Tracking and Manipulation . . . . . . . . . . . . 5

1.4. Thesis Overview . . . . . . . . . . . . . . . . . . . . 6

CHAPTER 2. Foundations: Static NRT Analysis and Manipulation . . . . . . . . 9

2.1. Related Work . . . . . . . . . . . . . . . . . . . . . 10

2.2. NRT Analysis . . . . . . . . . . . . . . . . . . . . . . . . . 14

2.2.1. Geometric Deformation Field Extraction . . . . . . . . . . . . . . 15

2.2.2. Lighting Deformation Field Extraction . . . . . . . . . . . . . . . 17

2.2.3. Texture Regularity Measurement . . . . . . . . . . . . . . . . . . . 17

2.3. NRT Synthesis . . . . . . . . . . . . . . . . . . . . . . . . . . . . . . . . . . . . . . . 19

2.3.1. Type I NRT Synthesis . . . . . . . . . . . . . . . . . . . . . . . . . . . . 19

2.3.2. Type II NRT Synthesis . . . . . . . . . . . . . . . . . . . . . . . . . 21

2.3.3. Type III NRT Synthesis . . . . . . . . . . . . . . . . . . . . . 23

2.4. NRT Manipulation . . . . . . . . . . . . . . . . . . . . . . . . 25

2.4.1. Texture Regularity Manipulation . . . . . . . . . . . . . . . . . . . 25

2.4.2. Texture Replacement in Real Photos . . . . . . . . . . . . . . . . . . . 25

2.5. Texture Synthesis Algorithm Comparison for NRT Synthesis . . . . . . . . . 34

2.5.1. A Brief Review of Selected Algorithms . . . . . . . . . . . . . . . . . . 34

2.5.2. Analysis of Comparison Results . . . . . . . . . . . . . . . . . . . 36

2.5.3. Summary of the Comparison Study . . . . . . . . . . . . . . . . 46

CHAPTER 3. Dynamic NRT Modeling, Tracking and Manipulation . . . . . . . . 53

3.1. Related work . . . . . . . . . . . . . . . . . . . . . . 55

3.1.1. Dynamic Textures Analysis and Synthesis . . . . . . . . . . . . . . . 56

3.1.2. Visual Tracking . . . . . . . . . . . . . . . . . . . . . . . . . . 57

3.1.3. Garment Motion Capture . . . . . . . . . . . . . . . . . . . . . . . 59

3.2. Introduction to Dynamic NRTs . . . . . . . . . . . . . . . . . . . . 60

3.3. Challenges of Tracking and Manipulating Dynamic NRTs . . . . . . . . . 61 
3.3.1. Ambiguous Texton Correspondences . . . . . . . . . . . . . . . . 62

3.3.2. Temporal Variations . . . . . . . . . . . . . . . . . . . 63

3.3.3. Occlusions . . . . . . . . . . . . . . . . . . . 63

3.3.4. Temporal Coherence . . . . . . . . . . . . . . . . . . 64

3.3.5. Unknown Camera Parameters . . . . . . . . . . . . . . . . . . . . . . 64

3.4. Mathematical Model of Dynamic NRTs . . . . . . . . . . . . . . . . . . . . 65

3.4.1. Texton Geometry Model . . . . . . . . . . . . . . . . . . . . . . . . 65

3.4.2. Lattice Structure Model of a Dynamic NRT . . . . . . . . . . . . . . . 67

3.4.3. Spatial Tracking-Texton Detection . . . . . . . . . . . . . . 72

3.4.4. Temporal Lattice-based MRF model . . . . . . . . . . . . . . . . . 73

3.5. Dynamic NRT Tracking . . . . . . . . . . . . . . . . . . . . . 74

3.5.1. Tracking Initialization and Texton Detection . . . . . . . . . . . 75

3.5.2. Spatial Inference . . . . . . . . . . . . . . . . . . . . 76

3.5.3. Temporal Tracking . . . . . . . . . . . . . . . . . . 78

3.5.4. Template Update . . . . . . . . . . . . . . . . . . . . . . . . . . . 79

3.5.5. Results . . . . . . . . . . . . . . . . . . . . . . . 79

3.5.6. Validation and Comparison . . . . . . . . . . . . . . . . 84

3.6. Dynamic NRT Manipulation . . . . . . . . . . . . . . . . . . . . . . . . 95

3.6.1. Dynamic NRT replacement . . . . . . . . . . . . . . . . . . . 997

3.6.2. Video Superimposing . . . . . . . . . . . . . . . . . . . . . . . . . . 102

3.6.3. NRT Video Synthesis . . . . . . . . . . . . . . . . . . . 102

CHAPTER 4. Conclusion . . . . . . . . . . . . . . . . . . . . . . . . 109

4.1. Contributions . . . . . . . . . . . . . . . . . . . . . 109

4.2. Limitations . . . . . . . . . . . . . . . . . . . . . . 110

4.2.1. Static NRT analysis and manipulation . . . . . . . . . . . . . 110

4.2.2. Dynamic NRT Modeling, Tracking and Manipulation . . . . . . . . 110

4.3. Potential Applications and Future Extensions . . . . . . . . . . . . . 111

4.4. Summary . . . . . . . . . . . . . . . . . . . . . 112

BIBLIOGRAPHY . . . . . . . . . . . . . . . . . . . . . . 113

APPENDIX A. Piecewise-affine Alignment of Textons . . . . . . . . . . . . . . 121

A.1. Review of Lucas-Kanade Algorithm . . . . . . . . . . . . . . . . . . . 121

A.2. Piecewise Affine Alignment for Tightly Coupled Textons . . . . . . . . . 122

A.3. Affine Alignment for Loosely Coupled Textons . . . . . . . . . . . . . . 123

APPENDIX B. NRT Synthesis Comparison Results . . . . . . . . . . . . . . . . 125 


\section{LIST OF FIGURES}

$1.1 \quad$ A texture spectrum on which textures are arranged according to their geometric regularity. . . . . . . . . . . . . . 2

1.2 There are only five possible types of tiles in 2D regular textures. (figure modified from [Liu et al., 2005]) . . . . . . . . . . . . . . 3

$1.3 \quad$ Examples of near-regular textures. . . . . . . . . . . . . . 4

1.4 Snapshots of three dynamic NRTs. These images show the challenges of dynamic NRT tracking: (a) self and external occlusion; (b) rapid movement and illumination change; (c) varied forms. . . . . . . . . 6 Overview of dynamic NRT replacement. . . . . . . . . . . . 7 Dynamic near-regular texture replacement results of our algorithm.

$2.1 \quad$ Examples of three types of near-regular textures. . . . . . . . . . 15

(a) Regular lattice placed over the input texture based on user input of two translation vectors $\mathbf{t}_{1}, \mathbf{t}_{2}$. (b) Near-regular lattice resulting from user adjustment of lattice points. (c) The straightened lattice [Liu et al., 2004]. . . . . . . . . . . . . . . . . . . . . . . . . 15

2.3 (a) input texture (b) input texture with a user-identified lattice (c) straightened texture $(\mathrm{d})$ extracted geometric deformation field (green mesh) and lighting deformation field [Liu et al., 2004]. . . . . . . . . 18

2.4 Textures plotted using our geometric $(\mathrm{G})$ and appearance (A) regularity measures. The higher the scores, the more irregular the texture. Notional classification regions for Type I,II and III near-regular textures are indicated [Liu et al., 2004]. . . . . . . . . . . . . . . . 19

2.5 (a) Illustration of maximum tiles (yellow rectangles) (b) The maximum tile set extracted based on lattice in (a) [Liu and Tsin, 2002]. . . . . . 20

2.6 An overview of the geometric deformation field synthesis [Liu and Lin, 2003] and manipulation algorithm. Starting with an input near-regular texture (NRT) $\mathcal{P}$, its geometrically regular version $\mathcal{P}_{I}$ is obtained by "straightening out" its underlying lattice $L$ into the nearest regular lattice such that $L=d_{\text {geo }}\left(L_{r}\right)$ and $\mathcal{P}=d_{\text {geo }}\left(\mathcal{P}_{I}\right) . \mathcal{P}_{I}$ is a Type I NRT by definition and $d_{g e o}$, a geometric deformation field treated as a texture, is usually not a regular or near-regular texture. We synthesize $\mathcal{P}_{I}$ to $\widetilde{\mathcal{P}}_{I}$ using a Type I NRT synthesis algorithm developed in [Liu et al., 2005], and synthesize the deformation 
field $d_{g e o}$ to $\widetilde{\mathcal{D}}_{\text {geo }}$ based on the algorithm proposed in [Efros and Leung, 1999]. Finally, the NRT manipulation is achieved by applying different gains to $\widetilde{\mathcal{D}}_{\text {geo }}$. When gain $=1$, the output texture is most similar to the input texture [Liu et al., 2004].

Type II NRT synthesis results. Left: input texture and extracted geometric deformation field. Center: synthesized geometric deformation field. Right: output texture [Liu and Lin, 2003]. . . . . . . . . . . . . 24

One of our texture-synthesis results compared with other methods on a type II NRT. One can observe on close inspection the regular alterations in color (... yellow, green, yellow, green, ...) of the input texture. This regularity is more faithfully preserved by our texture synthesis method (Figure 2.6, when gain =1). In the quantified geometry-appearance regularity space represented by the $G$ - $A$ regularity scores (Section 2.2.3), our output is the closest to the input texture [Liu et al., 2004]. . . . . . . . . . . . . . . . . . . . .

Formulation and result of deformation field analogy, where $A$ and $A^{\prime}$ are the geometry and lighting deformation fields respectively. $B$ is a synthesized geometric deformation field generated by the method in section 2.3.2. $B^{\prime}$ is an analogy result generated by image analogy [Hertzmann et al., 2001]. . . . . . . . . . . . . . . . 28

2.10 Type III near-regular texture synthesis results. Top left is the input texture. The geometric and lighting deformation fields of the input and synthesized textures are shown in Figure 2.9.

2.11 Results of manipulating the geometric regularity, where the magnitude of all vectors in the deformation field is uniformly scaled by the gain parameter, $\mathrm{G}$ is the geometric regularity of the texture. The bottom row shows the corresponding geometric fields. . . . . . . . . . 30

2.12 Texture replacement of a fabric texture on cloth (top left is the input

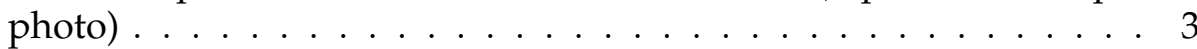

2.13 Texture replacement of a rose patterned cloth (top left is the input photo). The rest are texture replacement results of our method. Our method takes one input image and a target texture sample. If the target texture is near-regular, it is first synthesized using Type I nearregular texture synthesis before the deformation fields are applied. Deformation fields of the input image are shown in Figure 2.3. . . . 31

2.14 Texture replacement on an outdoor photo(top left). The lighting deformation field of the input texture is shown in Figure 2.15(b) . .

(a) Three complete vertical tiles of an NRT, each tile is three-floors high, are identified in an outdoor photo. Internal correspondences are added (each large tile is divided into three portions) for an extra assurance on global as well as local geometric consistency during deformation. Less than 4 minutes are needed for the lattice extraction process, initiated by the user (Section 2.2.1) [Liu et al., 2004]. (b) Lighting deformation field. . . . . . . . . . . . . . . 33 
2.16 The g score errors of synthesized textures, where the $x$-axis is the figure number of the texture, and the $z$-axis is the g score difference between a synthesized texture and its original input texture. The numeric value of $\mathrm{g}$ score difference of each texture is listed in Table 2.3

2.17 The a score errors of synthesized textures, where the $x$-axis is the figure number of the texture, and the z-axis is the a score difference between a synthesized texture and its original input texture. The numeric value of the a score difference of each texture is listed in Table 2.3 . . . . . . . . . . . . . . . . . . . . . .

2.18 Tested textures in our comparison study. Textures 1 to 5 are regular textures and the others are type I NRTs. The numbers in the parentheses are the ga-scores of a texture. The synthesized textures are shown in Figure B.2 - B.14 (Appendix B) . . . . . . . . . . . . . . . 48

2.19 Tested textures in our comparison study (continue). The synthesized textures are shown in Figure B.2 - B.14(Appendix B) . . . . . . . . .

2.20 Pairwise comparison of the near-regular synthesis approach with the graph cut approach (top), the regularized patch-based approach (middle), and the patch-based approach (bottom). In each plot, the center of each vertical line represents the mean of user scores of a texture given by 10 subjects and the lower and upper bound of the line is one standard deviation on each side of the mean. From the graph cut results (top plot) and the patch-based results (bottom plot), one can observe that the variations of user scores across different textures are larger than those across different users. It appears that the performance of the graph cut approach and the patch-based approach are less consistent on different NRTs than the performance of the near-regular synthesis approach and the regularized patchbased approach. . . . . . . . . . . . . . .

$2.21 \quad$ Histogram of the user scores of all synthesized textures, where green bars represent those textures whose global regularity is preserved and blue bars represent those violated. The range of user score, from best to worst, is 4 to 1 . This plot shows that preservation of global regularity is an important factor in user evaluation since most of the green bars are located in the higher score region while most of the blue bars are located in the lower score region. However, this plot also shows that the regularity preservation is not the only factor that a user evaluates a synthesized texture since there are still few regularity-preserved synthesized textures receiving low scores and some regularity-violated synthesized texture receiving high scores. This discrepancy may be because that the regularity violation is not noticeable to some users or some users count on other factors more than regularity preservation. . . . . . . . . . . .

3.1 Examples of dynamic near-regular textures with tightly coupled textons. Textons are located on a deforming surface where there is no gap between neighboring textons. . . . . . . . . . . 54 
3.2 Examples of dynamic near-regular textures with loosely coupled textons. Neighboring textons are moved with loosely connected constraints. The texture at left is a pattern seen through disturbed water. . . . . . . . . . . . . . . . . . 54

3.3 This figure shows the lattices (red lines) and textons (yellow quadrilaterals) of two types of NRTs: tightly and loosely coupled textons. We model the lattice of an NRT as a 2D MRF where each node represents a

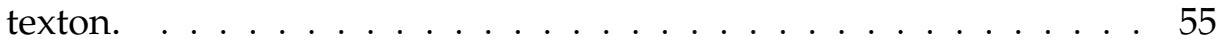

3.4 Garment motion capture system. (a) Camera setup: 8 cameras and 2 HMI lamps with softboxes. (b) A skirt with color-coded patterns used in the motion capture system. (c) Reconstructed surface. (d) Reconstructed surface with texture mapping. Pictures taken from [Scholz et al., 2005]. . . . . . . . . . . . . . . 60

3.5 These images show the challenges of dynamic NRT tracking: ambiguous correspondences (left); temporal variations (top-right); occlusion (bottom-right). . . . . . . . . . . . . . . . 62

3.6 Texton geometric model. Red lines form the quadrilateral lattice structure of a dynamic NRT. $\mathbf{c}^{i 1}, \mathbf{c}^{i 2}, \mathbf{c}^{i 3}, \mathbf{c}^{i 4}$ denote image coordinates of the four vertices of the texton $i$. A texton is divided into two triangles (blue and yellow shaded), and the vertex coordinates of each triangle parameterize an affine transformation. (a) loosely coupled textons: the vertex positions of a texton are determined independently. (b) tightly coupled textons: the vertex positions of a texton are jointly determined by its neighboring textons (e.g., $\mathbf{c}^{i 4}$ is involved in six affine transformations). . . . . . . . . . 66

$3.7 \quad$ (a) The first frame of an dynamic NRT where a yellow quadrilateral represents a texton and red lines form the lattice. (b) Aligned textons based on textons in (a). These aligned textons serve as initial texton templates and are updated in successive frames. . . . . . . . . . . We view the lattice of a dynamic NRT as a network of springs that connect individual textons of a dynamic NRT. Yellow ovals represent textons.

(a) The cloth spring configurations in [Provot, 1995]. The yellow circles and lines represent mass particles and and springs respectively. There are 1) structural springs (red lines) connecting nearest-neighbor particle along vertical and horizontal lines, 2) shear springs (black lines) connecting a particle's nearest-neighbor particles along diagonals, and 3) flexion springs (purple lines) connecting a particle with its second neighbor along vertical and horizontal lines. (b) neighborhood configuration of our MRF model: a node is connected to twelve

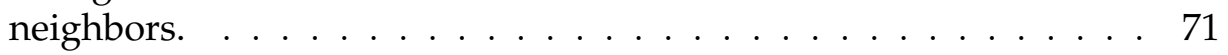

$3.10 \quad$ Illustration of our temporal lattice-based MRF model . . . . . . 74

$3.11 \quad$ Approach overview . . . . . . . . . . . 75

3.12 (a) Initial texton (yellow parallelogram formed by $\mathbf{t}_{\mathbf{1}}$ and $\mathbf{t}_{\mathbf{2}}$ ) and lattice (red lines). The neighboring textons are estimated by translating 
the first texton by $\mathbf{t}_{1},-\mathbf{t}_{1}$, and $\mathbf{t}_{\mathbf{2}}$. (b) Spatial prediction of the position of a new texton. . . . . . . . . . . . . . . . 76

3.13 Texton detection results by spatial tracking. Left: initial lattice.

Center: intermediate result. Right: final result. . . . . . . . . . . 80

3.14 Texton detection results by spatial tracking (a) Initial lattice. (b)(c) Intermediate results. (d) Final result. . . . . . . . . . . . . . . . 81

3.15 Lattice tracking results of a dynamic near-regular texture on waving cloth. One can observe that the tracking error accumulates quickly in the optical flow results.

3.16 Tracking results of an underwater texture at frame 1, 50, 91, and 100 (water_tracked.mov). Note that there are serious motion blurs and large lighting variations due to highlights in the video. Texture replacement results can be seen in water_replaced.mov. . . . . . . . 8 8

3.17 Tracking result of another underwater texture at frame 1,70,100 and 200. There are serious motion blurs and large lighting variations due to highlights in the video. . . . . . . . . . . . . . . . . .

3.18 Tracking results at frame 10, 110, 210, and 310 (crowd.avi). (a) Robust optical flow tracking results. (b) Lucas-Kanade tracking results using affine transformation parameters. (c) Our tracking results. . . . . . 86

3.19 Left column: tracking results of [Guskov, 2002] (Courtesy of Igor Guskov). Right column: our tracking results. This example shows that our tracking algorithm achieves similar performance with Guskov's algorithm that is specially designed for black-white-square patterns. The root mean square error of Guskov's results and ours against hand-labeled ground truth are 2.94 and 2.57 pixels respectively. . . 87

3.20 Tracking results of a fabric pattern under occlusion (dress.avi). The visible lattice, occluded lattice, visible textons, and occluded textons are shown in red, cyan, yellow, and cyan color. The visibility map shows visible aligned textons.

Top row: tracking results of a folding fabric pattern (towel.mov). There are a few textons totally occluded in the middle and two textons are occluded by a finger in the lower-right region. Bottom row: visibility map . . . . . . . . . . . . . . . . 88

3.22 Tracking results of an underwater texture at frame 25,50,75, and 100 (numNbrsExp.avi). . . . . . . . . . . . . . .

3.23 Plot of number of PCA bases (95\% energy) used to represent texton templates in the tracking process. At the first frame, only one basis is used since a single texton template is used. As tracking procee . . .

3.24 Comparison of tracking results using multiple texton templates (left column) and single texton template (right column). One can observe that the tracking results of multiple texton templates are more accurate. . . . . . . . . . . . . . . . . . . . . 90

3.25 Texton detection results starting at different initial positions. (a)(c)(e)(g) are initial textons and $(b)(d)(f)(h)$ are texton detection results correspondingly. Two textons at the top-left corner in (d) are not detected successfully 
because the image intensities of these textons are much darker than those of the initial texton. . . . . . . . . . . . . . . . 92

Texton detection results starting at different initial positions. (a)(c)(e)(g) are initial textons and $(b)(d)(f)(h)$ are texton detection results correspondingly. 93 A short image sequence showing the drifting of lattice in the AAM tracking result. The lattice drifts because the AAM is not able to local nonlinear deformation and tracking can not be recovered once the lattice drifts to other texton locations. . . . . . . . . . . . . . . 95

3.28 Comparison of multi-target tracking result (courtesy of Ting Yu and Ying Wu [2005]) and our tracking result at frame 10, 110, 210, and 310. Our tracking algorithm is able to keep tracking all targets through the entire sequence while $\mathrm{Yu}$ and Wu's algorithm may lose tracking of targets at short periods. . . . . . . . . . . . . . . 96

Comparison of root mean square error against the hand-labeled ground truth. The red dash line and blue line corresponds to the RMSE curve of $Y u$ and $W u$ 's and our tracking result. The total RMSE of $\mathrm{Yu}$ and $\mathrm{Wu}$ 's results and ours are 25.9 and 20.2 pixels respectively. Overview of dynamic NRT replacement. . . . . . . . . . . . 98

3.31 Comparison of lighting DF without and with smoothing. Note that only smoothing a light DF spatially cannot totally remove "holes" in the original DFs. . . . . . . . . . . . . . . . . 100

3.32 Texture replacement result of a pattern seen through disturbed water (water_replaced.mov). (a) Input video at frame 1, 79, 82, 91 and 100. (b) Texture replacement with a brick texture. (c) Texture replacement with a Monalisa's image. . . . . . . . . . . . . . . . 103

3.33 Tracked lattices and extracted lighting DFs from a fabric texture. . . 104 Texture replacement results of a fabric texture on trousers. . . . . 105 Video superimposing result under self-occlusion (superimpoing.avi). One can observe that the top-right corner of the letter ' $U$ ' preserves the occlusion effect in the input video. . . . . . . . . . . . 106 This figure shows an overview of video texture synthesis algorithm. The motion field of an input video is obtained by the dynamic NRT tracking algorithm. A new motion of lattice points is then generated by the 3D DF synthesis algorithm and is used to retrieve images from the input video. The retrieved images are warped and combined to generate the output video. . . . . . . . . . . . . . 107

3.37 Dynamic NRT synthesis example 1: synthesizing from a single motion field. . . . . . . . . . . . . . . . . . . . 108

3.38 Dynamic NRT synthesis example 2: synthesizing from two motion fields by summation. . . . . . . . . . . . . . . 108

4.1 It is possible to apply our tracking algorithm to other types of data that demonstrate lattice property. This figure shows a tagged MRI image of a heart. Those tagged lines in the image form a lattice structure. . . . . . . . . . . . . . . . . . . . 112 
B.1 This figure shows the results due to different settings for patch placement in the graph cut approach. In the synthesis process, an error map is computed using seam cost around each pixel and then the location around which we want to paste the new patch is picked by sampling from this error map. The results shown are obtained respectively by (b)picking the pasting location randomly, (c)computing a local minima for these error values and picking a location from one of these, (d)computing both local minima and maxima and picking a location from one of these. The synthesis result of the same texture by the near-regular synthesis approach is shown in Figure B.12 (Texture 34). . . . . . . . . . . . . . 125

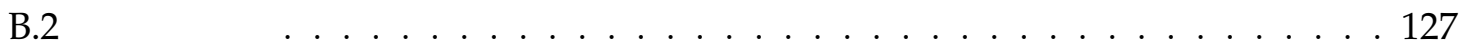

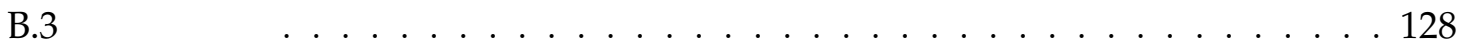

B.4 $\quad \ldots \ldots \ldots \ldots 12 \ldots \ldots \ldots$

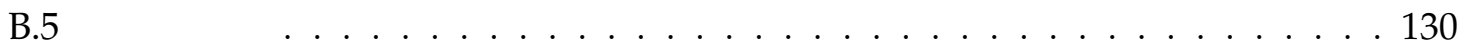

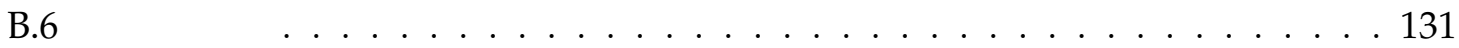

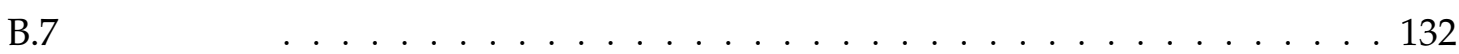

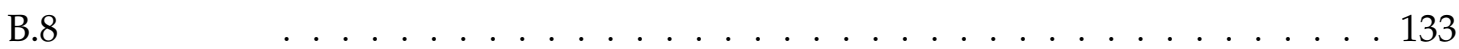

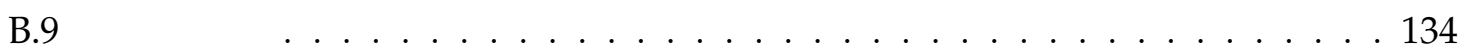

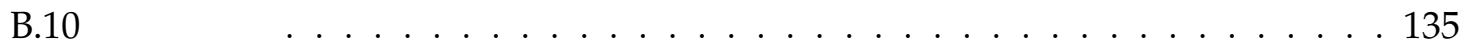

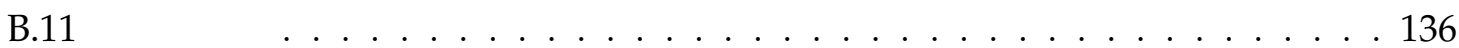

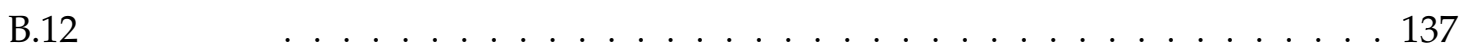

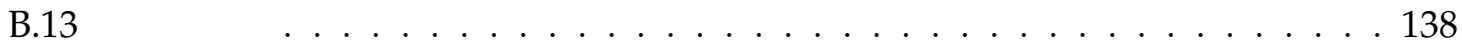

B.14 . . . . . . . . . . . . . . . . . . . . . . . . . . 139

B.15 Texture synthesis results of image quilting [Efros and Freeman, 2001].140

B.16 Texture synthesis results of image quilting [Efros and Freeman, 2001].141 



\section{LIST OF TABLES}

2.2 Summary of four synthesis algorithms. A patch is a 2D sample of neighboring pixels extracted from the input texture image. Patch shape/size and extraction refer to how the shape and size of the region are determined, and where each patch is located in the input texture. Patch placement and stitching refer to how the patches are placed and stitched in the synthesized texture. . . . . . . . . . . . 36

2.3 Results of regularity preservation test on regular textures (top) and near-regular textures (bottom), where $\checkmark$ denotes that regularity is preserved, and $\times$ denotes not. $g$ err. and $a$ err. are the difference of the geometry and appearance regularity between a synthesized texture and its input texture. The g score error with asterisk denotes that no lattice structure exists in the texture and the maximum score error is

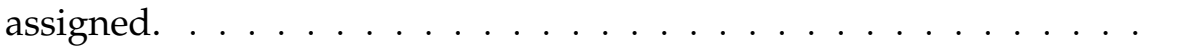

$2.4 \quad$ Results of user evaluation on regular textures (top) and near-regular textures (bottom), where $\checkmark$ denotes that regularity is preserved, and $x$ denotes not. The numbers in the table are the mean and standard deviation of scores given by 10 subjects. The range of score, from best to worst, is 4 to 1 . The standard deviations of the synthesized textures that are greater than 1.15 and less than 0.33 are shown in red texts and blue texts respectively. . . . . . . . . . .

Summary of the user evaluation test on four texture synthesis algorithms, including regular and near-regular textures. The mean and standard variation of each algorithm is computed across different users and textures. ANOVA method [Neter et al., 1996] (section 2.5.2) is used to verify if there is statistically significant difference between the mean of the near-regular synthesis algorithm and the means of the other three. We did pairwise comparisons and the differences of means in all three comparisons are statistically significant. . . . . . . . . . .

2.6 Comparison of user scores of regularity-preserved and regularityviolated textures. ANOVA method [Neter et al., 1996] (section 2.5.2) is used to verify if there is a statistically significant difference between the mean of the regularity-preserved synthesized textures and that of the regularity-violated synthesized textures. This table shows that 
synthesized NRTs that preserves global regularity get higher user scores and the difference is statistically significant. . . . . . . . . . 45 


\section{CHAPTER 1}

\section{Introduction}

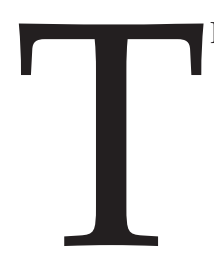

EXTURE originally refers to any natural structure having an appearance or consistence as if woven ${ }^{1}$. The definition of texture in computer vision and computer graphics, however, deviates from its original meaning. Texture can mean an image region that has non-uniform image intensities, images sharing the same statistics that cannot be told apart by visual perception [Julesz, 1962], or the surface appearance changes separated from an object's gross geometry [Turk, 1994]. In this thesis, texture is understood as an image that demonstrates regularity and randomness. The degree of regularity and randomness of different textures varies, ranging from strictly periodic patterns to stochastic noises. We view the variation of texture regularity as a continuous texture spectrum. Figure 1.1 illustrates a texture spectrum where textures are arranged according to their geometric regularity from left (most regular) to right (least regular).

Texture is a common phenomenon in the real-world that it has been studied in computer vision and computer graphics for many years [Julesz, 1962; Zucker, 1976; Yokoyama and Haralick, 1979; Perlin, 1985]. Texture analysis is applied to model textures [Chantler and Gool, 2005], and the acquired texture model can be used for many vision applications, such as object detection, segmentation, motion tracking, image retrieval, and classification. Texture synthesis has also been used widely in computer graphics to enrich the photorealism of a computer-generated virtual world. As the acquisition devices for images and videos become more popular, there are increasing

\footnotetext{
${ }^{1}$ Oxford English Dictionary. http:/ / dictionary.oed.com
} 


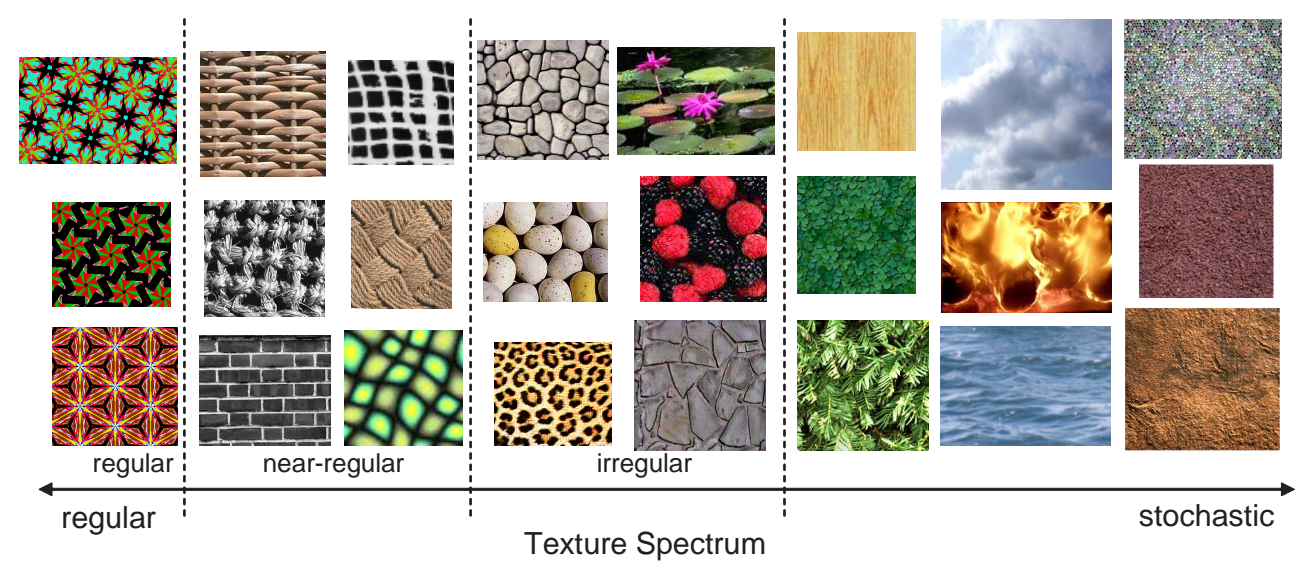

Figure 1.1. A texture spectrum on which textures are arranged according to their geometric regularity.

demands in image editing and video editing applications. Many of these editing techniques are texture-related. Therefore, a thorough study as well as an effective computational framework for textures is important for theoretical research and practical applications.

Realizing that a texture possesses both regularity and randomness is important when we develop algorithms to handle textures. One of the problems of the existing work on texture analysis and synthesis is that texture regularity has rarely been addressed. Although these algorithms work well on a variety of textures, they cannot handle those textures with strong regularity effectively. Liu and Tsin [2002] first pointed out the regularity preservation problem in the state of the art texture synthesis algorithm on what they termed near-regular texture (NRT). They showed that existing synthesis approaches cannot preserve the interlock structure in textures such as brick wall and woven fabric. They proposed an NRT synthesis algorithm [2002; 2005] that utilizes a lattice structure to model and maintain structural regularity in the synthesis process. Our previous work [Liu and Lin, 2003; Liu et al., 2004] further exploits texture regularity to analyze and manipulate NRTs by modeling an NRT as a deformed regular pattern in geometry and photometry from its regular origin.

Using [Liu et al., 2004] as a foundation in this thesis, we study dynamic NRTs. Specifically, we address the following problems: (1) how to model the geometry and appearance of a dynamic NRT; (2) how to track the global structure and individual textons of a dynamic NRT under occlusions and rapid movements; (3) how to replace a 


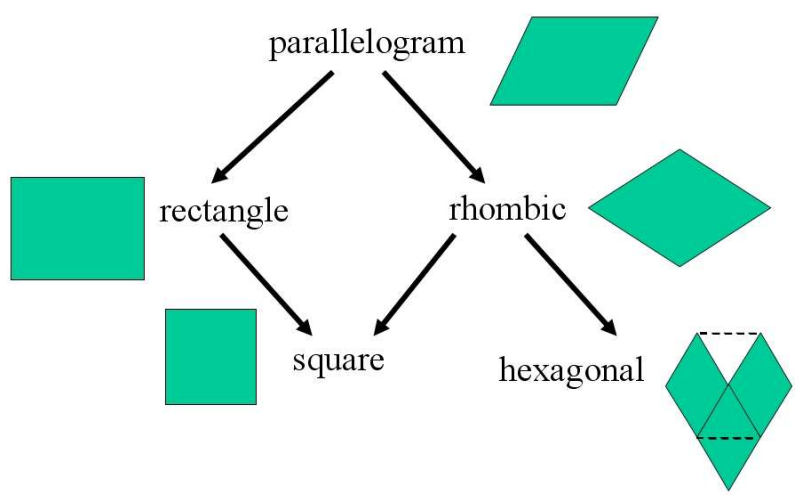

Figure 1.2. There are only five possible types of tiles in 2D regular textures. (figure modified from [Liu et al., 2005])

dynamic NRT with another texture in a video while preserving the original geometric and photometric visual effects as well as maintaining temporal coherence in the generated video.

\subsection{What is a near-regular texture?}

The origin of a near-regular texture is a wallpaper-like regular texture. A regular texture is a $2 \mathrm{D}$ wallpaper pattern generated by translating a parallelogram in two linear independent directions to cover (no gaps) and pack (no overlaps) two-dimensional space [Grünbaum and Shephard, 1987; Schattschneider, 1978; Liu et al., 2005]. We call the smallest such parallelogram the tile of the texture and the orbit of the center point of a tile under the two smallest translations $\mathbf{t}_{\mathbf{1}}$ and $\mathbf{t}_{\mathbf{2}}$ the lattice of the texture. Using $\mathbf{t}_{1}$ and $\mathbf{t}_{2}$, we can construct a parallelogram lattice in which each parallelogram corresponds to a tile. We call this parallelogram lattice the lattice of the regular texture. There has been a mature mathematical theory for wallpaper-like regular textures for over 100 years [Fedorov, 1885; Grünbaum and Shephard, 1987]-namely the wallpaper group theory. It has been shown that despite an infinite variety of regular textures, seventeen wallpaper groups are necessary and sufficient to completely characterize all possible structural symmetry of any 2D periodic pattern. There are only five possible lattice/tile shapes [Coxeter and Moser, 1979] and they form a tile shape hierarchy in which the parallelogram is the most general shape among the five (Figure 1.2)[Liu et al., 2005; Liu et al., 2004 PAMI]

Given the definition of regular textures, a near-regular texture is defined as a texture deformed geometrically and photometrically from its regular origin [Liu et al., 


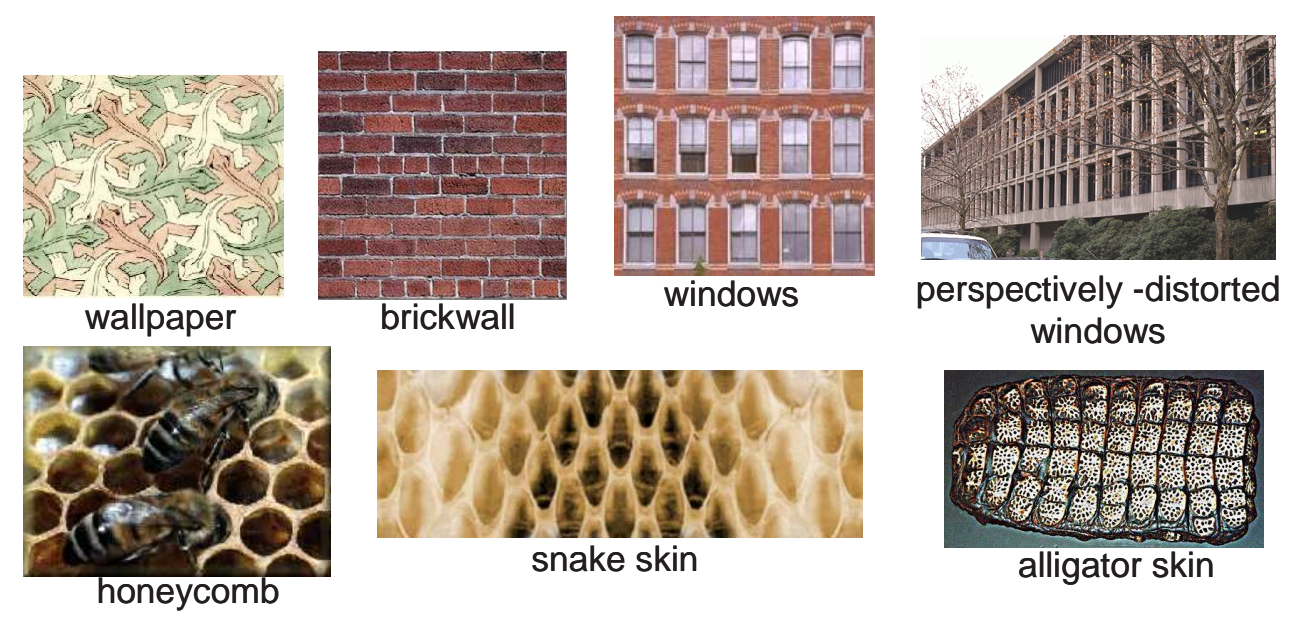

Figure 1.3. Examples of near-regular textures.

2004]. In its regular state, the geometric and topological structure of an NRT can be described by a 2D parallelogram lattice or a pair of translation vectors [Liu et al., 2004 PAMI] . When an NRT deforms geometrically from its origin, the parallelogram lattice may deform into a quadrilateral lattice, but its topology remains invariant. We call a quadrilateral on a lattice of an NRT a texton ${ }^{2}$. A texton can be considered as a tile under geometric and lighting deformation.

An NRT can be a single image, which we call a static NRT, or it can be an image sequence of a moving NRT, which we call a dynamic NRT. An important property of NRTs that distinguishes them from other types of textures is that its topology remains the same despite geometric or photometric deformations. Spatially, at each frame, the lattice structure relates a static NRT to its geometrically regular state such that the geometrical and photometrical properties of the NRT can be computed. Temporally, the lattice structure establishes the correspondences of a dynamic NRT at different frames such that the motion of the texture can be modeled, tracked and analyzed.

Conceptually, we can view the tile of a regular texture moves on a 2D plane under translational symmetry and the appearance of the tile is spatially invariant; a texton of a static NRT moves on a 2D surface but its appearance and geometry varies spatially; a texton of a dynamic NRT moves in 3D spatiotemporal space with varying geometry and appearance.

\footnotetext{
${ }^{2}$ Texton [Julesz, 1981] originally refers to fundamental micro-structures in natural images. It is considered as the atoms of pre-attentive human visual perception.
} 


\subsection{Static NRT Analysis and Manipulation}

The central idea of the NRT analysis and manipulation is that the regularity of an NRT is a continuous quantity that reflects the appearance variations of an NRT. These variations may occur in different subspaces, such as geometry, color, lighting, etc. Thus, an NRT can be considered to be a statistical distortion of a regular, wallpaper-like congruent tiling, and this statistical distortion can be represented by pixel-wise mapping, which we call a deformation field (DF).

In [Liu et al., 2004], we treat static NRTs geometric and lighting deformation fields as both a mapping and a texture on its own right. The functional property is used to deform an NRT, either in geometric or lighting space or both, while the textural property is utilized to synthesize new deformation fields. Based on geometric and lighting deformation fields, we are able to develop several manipulation functions on static NRTs in addition to conventional texture synthesis. These functions include texture regularity manipulation in geometry and color, and texture replacement (section 2.4).

\subsection{Dynamic NRT Modeling, Tracking and Manipulation}

A dynamic NRT exists in a 3D spatiotemporal space. The additional degree of freedom in temporal space introduces new challenges for handling a dynamic NRT. To deal with the problems related to a dynamic NRT, it is crucial to have an appropriate model for dynamic NRT.

Seeking for an effective computational tool and an in-depth understanding of dynamic NRTs, we propose a lattice-based MRF model for dynamic NRTs. The main concept of our model is that we treat textons of an NRT as multiple targets with a topological constraint while allowing individual textons to vary flexibly in geometry and appearance. Inspired by the physics-based cloth simulation [Provot, 1995; House and Breen, 2000; Choi and Ko, 2002], we model the lattice topology as a network of springs and implement it as an MRF. The implementation is based on the intuition that the operation of an MRF resembles the behavior of a network of springs in statistical fashion. We use a Lucas-Kanade-registration-based observation model to handle the appearance and geometry variations of individual textons in the tracking process.

The goal of dynamic NRT tracking is to track the motion of a dynamic NRT, including the variations of the global structure and local individual textons. Dynamic 


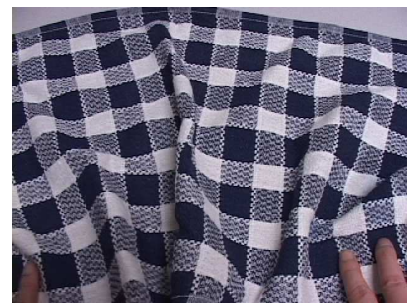

(a) folding towel

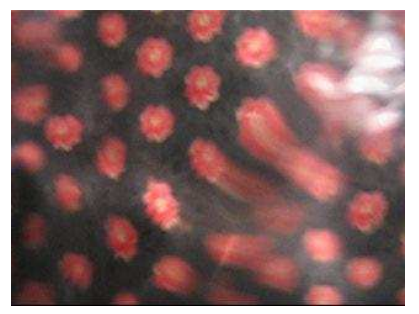

(b) underwater texture

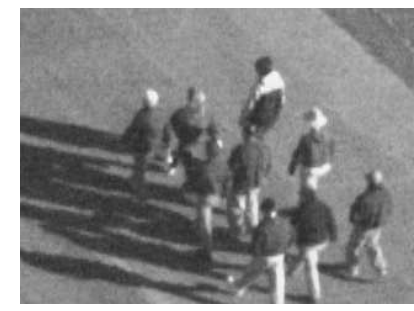

(c) crowd motion

Figure 1.4. Snapshots of three dynamic NRTs. These images show the challenges of dynamic NRT tracking: (a) self and external occlusion; (b) rapid movement and illumination change; (c) varied forms.

NRT tracking has many difficulties. A tracking algorithm can easily mistake one texton for another due to similar appearance of textons. Furthermore, the tracking problem becomes very difficult when the textons of a dynamic NRT move rapidly or occlude each other on a folded surface. Dynamic NRT exists in varied forms; it may be a fabric pattern on moving cloth, a texture being seen through disturbed water, or even the motion of a crowd. Due to these difficulties, tracking a dynamic NRT remains an unsolved problem. Figure 1.4 shows static frames of three different NRTs. This figure demonstrates the challenges of the tracking problem. Despite the challenges of dynamic NRT tracking, we develop a lattice-based tracking algorithm based on the proposed dynamic NRT model. We treat the dynamic NRT as a spatiotemporal inference formulation and solve the problem using the belief propagation and the particle filtering algorithms.

Tracking a dynamic NRT is important for modeling a dynamic NRT because it captures the motion of a dynamic NRT and a sequence of geometric and lighting DFs can be computed based on the tracked lattices. With the assistance of a dynamic NRT tracking algorithm, we are able to model geometry and lighting variations of a dynamic NRT temporally. More importantly, we can utilize the acquired deformation fields to manipulate a dynamic NRT. This creates the possibilities of many novel applications, such as texture replacement in videos, video superimposing, video texture synthesis, and cloth motion capture. Figure 1.5 is an overview of the proposed dynamic NRT replacement algorithm and a texture replacement result of our algorithm is shown in Figure 1.6.

\subsection{Thesis Overview}

The rest of the thesis is organized as follows: 


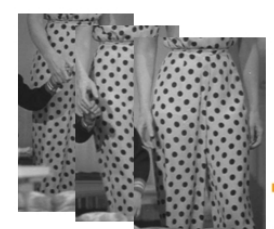

Input video

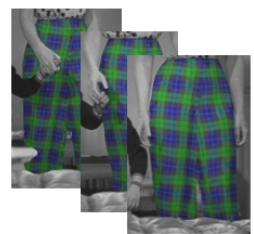

Output video

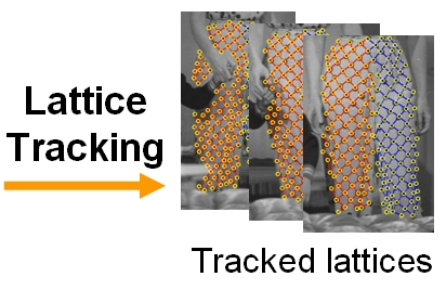

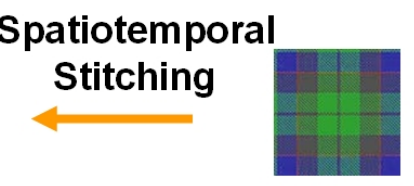

New texture

\section{Deformation \\ Field \\ Extraction}
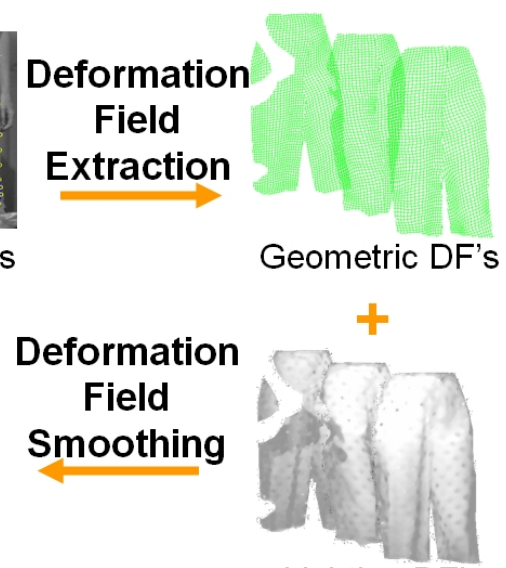

Lighting DF's

Figure 1.5. Overview of dynamic NRT replacement.
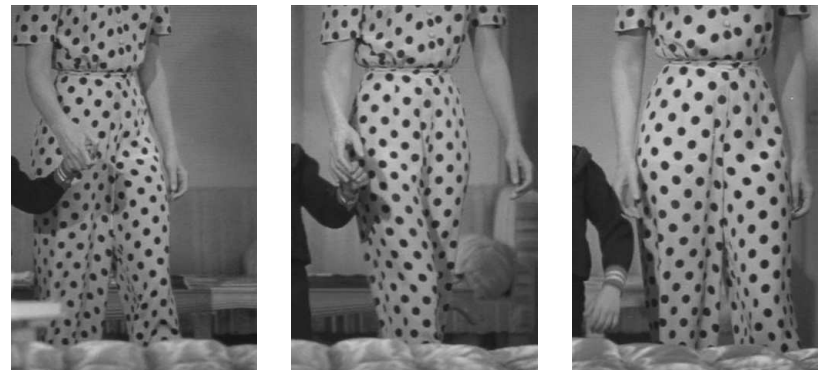

Input video.
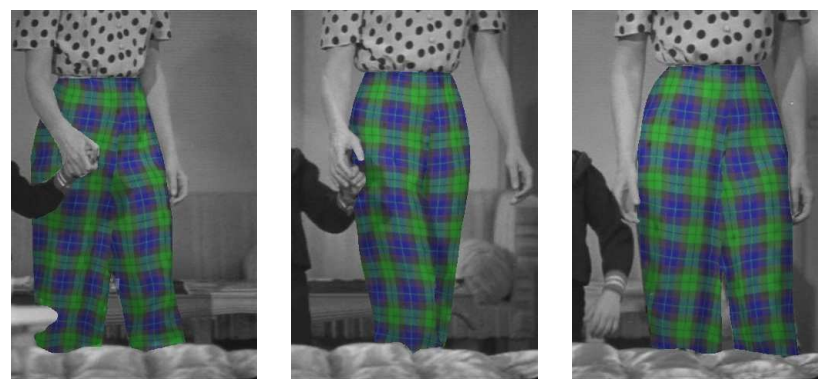

Output video
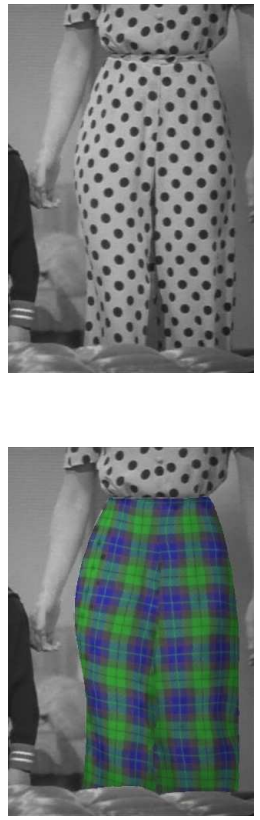

Figure 1.6. Dynamic near-regular texture replacement results of our algorithm.

\section{Chapter 2-Foundations: Static Near-regular Texture Analysis and Manipulation} For completeness, we review our previous work [Liu et al., 2004] on texture synthesis and manipulation of static NRT in this chapter. We introduce a lattice-based computational model for NRT analysis, synthesis, and manipulation. We also show how to quantitatively measure the texture regularity of an NRT and use the metrics to evaluate 
NRT synthesis results. To evaluate the performance of existing texture synthesis algorithms on NRT, we conduct a systematic comparison study where four texture synthesis algorithms are tested on 43 typical NRTs.

\section{Chapter 3-Dynamic Near-regular Texture Modeling, Tracking and Manipulation}

We review previous work on dynamic texture analysis and synthesis and visual tracking in this chapter. We then define the scope of dynamic NRTs and discuss the challenges of the problem. We present a dynamic NRT tracking algorithm that combines belief propagation and particle filtering to solve a spatiotemporal inference problem. Texture regularity is exploited to handle temporal feature correspondence and selfocclusion problems in dynamic NRT. We demonstrate the effectiveness of our algorithm on tracking dynamic NRTs under rapid movements, folding motion or illumination changes through different medium. We also present a dynamic NRT replacement algorithm that combines the spatiotemporal deformation field smoothing and spatiotemporal stitching to handle the temporal coherence problem. We demonstrate applications of our algorithm in NRT-based video editing.

\section{Chapter 4-Conclusion}

We discuss potential applications and future work for NRT. We also summarize the contributions of this thesis work to the field of computer vision and computer graphics in general, and for texture synthesis and video editing in particular. 


\section{CHAPTER 2}

\section{Foundations: Static NRT Analysis and Manipulation}

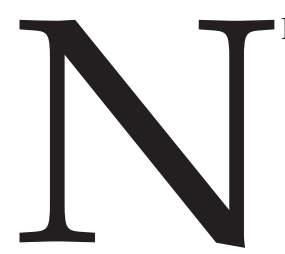

EAR-REGULAR TEXTURES exist in many man-made and natural scenes, e.g., wallpapers, windows, columns, brick wall, fabrics, honeycombs, animal skins, reptile scales, and others. An important feature of nearregular textures is that they exhibit regularity and randomness simultaneously. Examples of regularity include the interlocked structure of a brick wall, the interwoven structure of fabrics, and color alternation on reptile skins. The major challenge of NRT analysis and synthesis is how to faithfully model and reproduce both the global regularity and local randomness of an NRT.

In this chapter, we introduce a lattice-based framework [Liu et al., 2004] for analyzing and manipulating static NRTs. In this framework, a near-regular texture is considered as geometric and photometric departures from a regular congruent tiling. Treating regular tiling as the origin and with user-assisted lattice extraction, the geometry and lighting deviations of a near-regular texture are explicitly modeled as a pair of mapping functions, which we call geometric and lighting deformation fields. An important insight is that a deformation field plays a dual role as both a function that acts on a texture and as a texture that is acted upon. Each deformation field is thus subject to analysis, synthesis and manipulation. Using this formalization, the regularity and randomness of an NRT can be faithfully synthesized and purposefully controlled. To systematically and quantitatively justify that existing synthesis algorithms cannot respect regularity/randomness in NRTs, we compare our texture synthesis algorithm against four algorithms on 43 near-regular textures. Both the quantitative evaluation and user evaluation show that our synthesis algorithm achieves better performance. 


\subsection{Related Work}

We review related work in texture synthesis and texture replacement in this section.

Procedure-based Approaches. Procedure-based approaches synthesize textures by directly producing complex, interesting patterns using a program executed before or during the rendering process [Ebert et al., 2002]. The procedure can be based on a mathematical description. For instance, basis functions of band-limited noise [Perlin, 1985] were used to generate a variety of textures, such as fire, marble, and wrinkles. The procedure can also be based on a physical simulation, e.g., reaction diffusion [Turk, 1991; Witkin and Kass, 1991] was used to model biological patterns, such as fur, skin, and scales. Furthermore, detailed physical and chemical simulation [Dorsey et al., 1999] was used to reproduce some weathering and mineral phenomena. Although many near-regular textures can be synthesized by the procedure-based approach, it can be difficult to tune the parameters to cover a large set of near-regular textures.

Statistical-model-based Approaches. Statistical-model-based approaches [BarJoseph et al., 2001; Bonet, 1997; Heeger and Bergen, 1995; Portilla and Simoncelli, 2000; Zhu et al., 1998] are closely related to texture analysis and classification in that a statistical model is constructed based on an input texture and this model is then used for texture analysis or synthesis. For texture synthesis, a synthesized image is iteratively adjusted by matching statistical measurements of the synthesized image with those of the texture sample. Zhu et al. [Zhu et al., 1998] modeled texture as a Markov Random Field (MRF) and used Gibbs sampling for synthesis. The application of their approach is limited because Gibbs sampling is notoriously slow and it is difficult to assess when the sampling process has converged. Heeger and Bergen [Heeger and Bergen, 1995] gradually modified a random noise image to imitate the appearance of a texture sample by matching filter response histograms of the two images at different spatial scales. Similarly, Portilla and Simoncelli [Portilla and Simoncelli, 2000] also started from a noise image but their matching functions were based on the first and second order statistics of joint wavelet coefficients at adjacent spatial locations, orientations and scales. Instead of starting from a random noise image, De Bonet [Bonet, 1997] jumbled an input image in a coarse-to-fine fashion and randomizes it such that only the statistics to be matched were preserved. 
In general, statistics-model-based approaches perform much better on stochastic textures than on structural textures [Heeger and Bergen, 1995]. One of the reasons is that although the statistical measurements can model the characteristics of stochastic textures very well, they cannot capture the structural information of structural textures, especially the high frequency details of highly structured patterns [Efros and Leung, 1999].

Image-based Approaches. Image-based approaches [Ashikhmin, 2001; Efros and Freeman, 2001; Efros and Leung, 1999; Kwatra et al., 2003; Liang et al., 2001a; Wei and Levoy, 2000; Zhang et al., 2003] synthesize textures by directly copying image pixels or patches from an input texture and stitching them together in a synthesized image. Efros and Leung [Efros and Leung, 1999] proposed a nonparametric approach to synthesizing textures by directly sampling pixels from the input textures. They assumed that the formation of a texture is a Markov random field process where the probability density function of a pixel's intensity depends on those of its neighboring pixels. Therefore, a texture can be synthesized by adding pixels to the output image, where each pixel is picked from the input texture by comparing the similarity of its neighboring pixels to those existing in the synthesizing image. Several synthesis algorithms based on Efros and Leung's pixel-based approach have since been proposed. One of the driven forces for developing these variants was to reduce the computational cost of the pixel-based approach. Wei and Levoy [Wei and Levoy, 2000] proposed a fast synthesis algorithm by applying a tree-vector quantization technique to speed the search process and they extended their algorithm to texture synthesis on surfaces [Wei and Levoy, 2001] later. Ashikmin [Ashikhmin, 2001] observed from Wei and Levoy's algorithm [Wei and Levoy, 2000] that the pixels in the input texture sample with neighborhoods are similar to shifted current neighborhoods in the output image. This neighboring similarity property can be used to reduce the search space and significantly improve the synthesis speed.

After Ashikmin's work [Ashikhmin, 2001], the MRF concept of pixel-based approaches was further extended to the patch-based approaches where the texture is synthesized by growing patches instead of pixels [Efros and Freeman, 2001; Liang et al., 2001a; Nealen and Alexa, 2003; Kwatra et al., 2003]. Intuitively, approaches of this class can be considered to synthesize textures by a series of copying and pasting operations 
on image patches. These patches might be a fixed-size rectangular window [Efros and Freeman, 2001; Liang et al., 2001a], an adaptive square window [Nealen and Alexa, 2003] or an irregular window [Kwatra et al., 2003]. In addition, different stitching techniques were applied to remove the seams at the overlapped patches, including dynamic programming [Efros and Freeman, 2001], graph cuts [Kwatra et al., 2003], imagefeathering [Liang et al., 2001a], and pixel-based overlap re-synthesis [Nealen and Alexa, 2003]. To better preserve structural features in textures, $\mathrm{Wu}$ and $\mathrm{Yu}$ [2004] proposed a patched-based synthesis algorithm guided by image feature maps extracted from the input texture. In synthesis process, neighboring patches are warped so that features in neighboring patches are aligned. This reduces the presence of broken features and related artifacts in synthesized textures.

Image-based approaches produce very appealing synthesis results on many structural textures. In particular, image details can be well preserved in synthesized textures because the image-based approach tries to keep the image pixels untouched as much as possible.

There are also increasing research interests to allow more user controls involved in texture synthesis process. Matusik et al. [2005] used a simplicial complex model to represent the space of a texture database. The user can generate new textures by navigating the texture space with a morphable interpolation model. Lefebvre and Hoppe [2005] presented a real-time parallel synthesis algorithm that enables intuitive user controls such as multiscale randomness, spatial modulation, feature drag-and-drop, and periodicity constraints. Kwatra et al. [2005] proposed a synthesis algorithm that can be guided by a vector field. These synthesis approaches extend the applications of texture synthesis.

Near-regular Texture Synthesis. Although existing texture synthesis algorithms can faithfully synthesize many types of textures, there is a portion of the texture spectrum (Figure 1.1) from regular to stochastic that has not been well tackled. Liu and Tsin [Liu and Tsin, 2002] suggest that there is a need to develop a texture synthesis algorithm for this type of texture: near-regular texture. They had pointed to some results in [Efros and Freeman, 2001] where the regularity of the input texture was not faithfully preserved, then developed a new algorithm for faithful synthesis of near-regular 
textures. They analyzed an input texture and found its underlying lattice, either automatically or by providing two lattice generating vectors that provide the shape, size and orientation information of a tile. The lattice information was then used to extract a set of tiles that are of the same shape and size, but statistically varied in color and intensity. Their synthesis process is similar to the image quilting approach [Efros and Freeman, 2001] except that patches are picked from the analysis of the tile set and pasted around the lattice points to stitch into the existing synthesized image. In our recent study [Lin et al., 2004], we systematically compared four texture synthesis algorithms on 43 regular and near-regular textures. The results convincingly demonstrated that near-regular texture remains a challenge for several state-of-the-art algorithms (section 2.5).

Texture Replacement. Tsin et al. [2001] proposed a texture replacement algorithm that can replace near-regular patterns on a plane in real images. They assumed that the surface is planar and the texture is close to regular and a color/intensity model of the texture can be constructed by sampling multiple texture elements from a "canoni$\mathrm{cal}^{\prime \prime}$ lighting region. This model is used to detect the lighting variation and non-texture regions (segmentation) across the image. We have used a similar lighting and color model in our work; however, we relax their planar constraint to achieve texture replacement on non-planar surfaces [Liu et al., 2004].

The texture transfer effects shown in Image Analogy [Hertzmann et al., 2001] and Image Quilting [Efros and Freeman, 2001] bear some resemblance to NRT replacement. The difference is: (1)we explicitly treat both distorted geometry and the lighting effects as deformation fields so that we can synthesize them separately or jointly, while they model the association of data in intensity only; and (2)our method preserves the implied underlying (distorted) regular tiling of a near-regular texture, rather than its local appearance alone.

Besides the approaches that mainly operate on image space, there are approaches that recover the surface geometry in an image and map a new texture on the recovered surface. Lobay and Forsyth [2004] applied a shape-from-texture algorithm [Forsyth, 2002] to recover the shape and irradiance map from the textured surface of cloth. The shape and irradiance map are used for texture replacement. They did not handle the occlusion problem nor acknowledge the topological structure of an NRT in their work. 
Oh et al. [2001] also dealt with texture replacement in their photo editing system. They recovered the depth information in the image with user assistance. Depth information is used to generate foreshortening distortions and to extract the lighting variation in a textured region. They assumed large-scale variations are due to the lighting and small scale details are due to the texture, limiting them from capturing detailed shadows or large-scale NRTs, e.g., building windows (Figure 2.14).

Fang and Hart [2004] recovered a surface normal map of a non-textured surface in an image using a shape from shading algorithm. Their photo editing system allows the user to refine the normal map and adjust the texture orientation. They demonstrated texture synthesis and replacement results on complex and highly folded surfaces using their editing system; however, they do not deal with textured surfaces.

\subsection{NRT Analysis}

We view a near-regular texture as a statistical distortion of a regular, wallpaper-like congruent tiling, possibly with individual variations in tile shape, size, color and lighting. Near-regular textures can depart from regular tiling in different spaces. According to the space in which the regularity varies, we categorize near-regular textures into three types. Type I NRTs are those in which geometric structure is regular but color appearance of individual tiles are irregular (GRCI). Type II NRTs are those in which geometric structure is irregular while the topological structure of the color appearance is regular (GICR). Type III NRTs are those in which the color, geometry and lighting appearance of individual tiles are irregular (GICI). We summarize the categorization in Table 2.1. Figure 2.1 and 2.4 show examples of each type.

Table 2.1. A Categorization of Near-regular Textures

\begin{tabular}{|c|c|c|c|}
\hline Type & Geometry & Color & Symbols \\
\hline 0 & Regular & Regular & GRCR \\
\hline I & Regular & Irregular & GRCI \\
\hline II & Irregular & Regular & GICR \\
\hline III & Irregular & Irregular & GICI \\
\hline
\end{tabular}

For a 2D regular texture, there exists a lattice that divides the texture into several identical parallelograms. This lattice is constructed by two linearly independent vectors, $t_{1}$ and $t_{2}$, which define the size of the parallelogram. There exists a smallest parallelogram such that a covering (no gaps) and a packing (no overlaps) of a 2D plane 


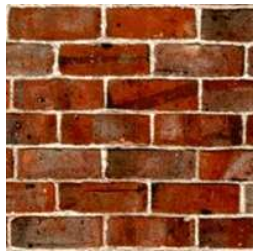

(a) Type I

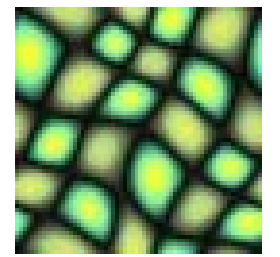

(b) Type II

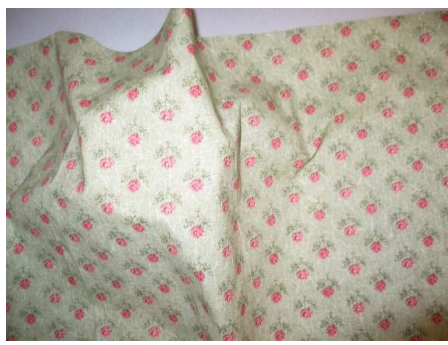

(c) Type III

Figure 2.1. Examples of three types of near-regular textures.

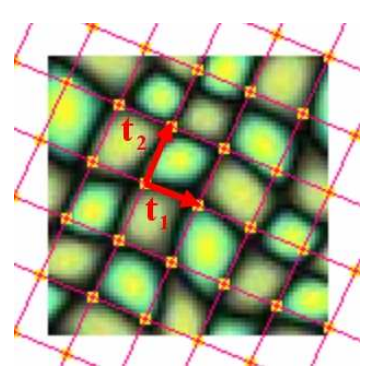

(a)

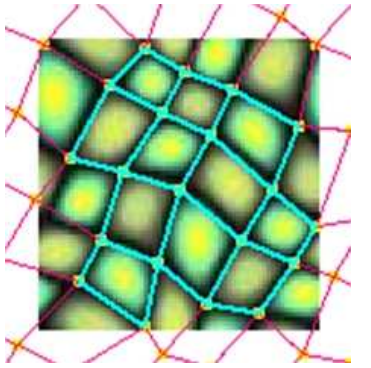

(b)

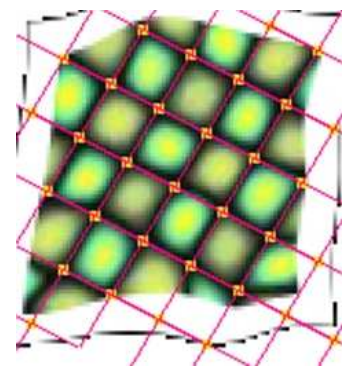

(c)

Figure 2.2. (a) Regular lattice placed over the input texture based on user input of two translation vectors $\mathbf{t}_{1}, \mathbf{t}_{2}$. (b) Near-regular lattice resulting from user adjustment of lattice points. (c) The straightened lattice [Liu et al., 2004].

can be constructed by translating the parallelogram on the plane based on $\mathbf{t}_{\mathbf{1}}$ and $\mathbf{t}_{\mathbf{2}}$. This parallelogram is called a tile. A near-regular texture is then defined as $\mathcal{P}=d\left(\mathcal{P}_{I}\right)$ where $\mathcal{P}_{I}$ is a Type I NRT and $d=d_{\text {geo }} \times d_{\text {light }}$ is a pixel-wise mapping representing the composition of geometric transformations and lighting changes respectively. The relation between type I, II, and III NRTs can be further described in terms of deformation fields: $\mathcal{P}_{I}=d_{\text {color }}\left(\mathcal{P}_{0}\right), \mathcal{P}_{I I}=d_{\text {geo }}\left(\mathcal{P}_{I}\right)$ and $\mathcal{P}_{I I I}=d_{\text {geo }}\left(d_{\text {light }}\left(\mathcal{P}_{I}\right)\right)$, where $\mathcal{P}_{0}, \mathcal{P}_{I}, \mathcal{P}_{I I}$ and $\mathcal{P}_{I I I}$ represent a type 0 (regular), I, II and III NRT respectively (Table 2.1).

\subsubsection{Geometric Deformation Field Extraction}

According to the definition of a NRT, every NRT $\mathcal{P}_{i}$ has an underlying lattice $L$, which is geometrically deformed from a regular lattice $L_{r}$ of a geometrically regular texture $\mathcal{P}_{I}$ (Figure 2.2(a)). Although there may be many potential regular lattices that a near-regular lattice $L$ can deform to, there is a well-defined regular lattice $L_{r}$ that has a minimum distance from $L$ [Liu et al., 2004 PAMI] . The geometric deformation field $d_{\text {geo }}$ is defined as a function that maps $L$ to $L_{r}$ and warps all the input texture pixels to a 
geometrically regular texture. The process of finding the geometric deformation field $d_{\text {geo }}$ is as follows:

Step 1 The user specifies two generating vectors $t_{1}$ and $t_{2}$ and the computer automatically constructs a regular lattice based on $\mathbf{t}_{\mathbf{1}}$ and $\mathbf{t}_{2}$ (Figure 2.2(a)).

Step 2 The user then adjusts the positions of some lattice points so that each tile is bounded by a quadrilateral on the deformed lattice (Figure 2.2(b)).

Step 3 The computer takes the user specified $L$ and automatically finds $L_{r}$, and establishes the correspondences between $L$ and $L_{r}$. Using the corresponding lattice points in $L$ and $L_{r}$ as control points, a pixel-wise warping function $d_{\text {geo }}$ between $\mathcal{P}$ and $\mathcal{P}_{I}$ is computed.

To determine the regular lattice $L_{r}$, we formulate the process as a minimization problem where the objective function is defined as the sum of the length variations of all corresponding link pairs between lattices $L$ and $L_{r}$.

$$
\min _{\left\|\mathbf{t}_{1}\right\|,\left\|\mathbf{t}_{\mathbf{2}}\right\|, \theta} E=\sum_{i=1}^{N_{i}}\left(l_{i}-\left\|\mathbf{t}_{\mathbf{1}}\right\|\right)^{2}+\sum_{j=1}^{N_{j}}\left(l_{j}-\left\|\mathbf{t}_{\mathbf{2}}\right\|\right)^{2}+\sum_{k=1}^{N_{k}}\left(l_{k}-\left\|\mathbf{t}_{\mathbf{1}}+\mathbf{t}_{\mathbf{2}}\right\|\right)^{2}+\sum_{m=1}^{N_{m}}\left(l_{m}-\left\|\mathbf{t}_{\mathbf{1}}-\mathbf{t}_{\mathbf{2}}\right\|\right)^{2}
$$

$l_{i}, l_{j}, l_{k}$, and $l_{m}$, are the lengths of the links in lattice $L$ corresponding to links in $L_{r}$ along the directions of $\mathbf{t}_{\mathbf{1}}, \mathbf{t}_{\mathbf{2}}, \mathbf{t}_{\mathbf{1}}+\mathbf{t}_{\mathbf{2}}$, and $\mathbf{t}_{\mathbf{1}}-\mathbf{t}_{\mathbf{2}}$, respectively. $N_{i}, N_{j}, N_{k}$ and $N_{m}$ are the total number of links in $L$ corresponding to each direction. $\theta$ is the angle between $\mathbf{t}_{\mathbf{1}}$ and $\mathbf{t}_{\mathbf{2}}$ which can be deduced from the lengths of $\mathbf{t}_{1}, \mathbf{t}_{\mathbf{2}}$ and $\mathbf{t}_{\mathbf{1}}+\mathbf{t}_{\mathbf{2}}$.

Once the optimal regular lattice $L_{r}$ is obtained, we can compute an image warping function between the near-regular texture and its geometrically regular version (Type I near-regular texture). We use the multilevel free-form deformation (MFFD) algorithm proposed by Lee et al. [Lee et al., 1995] to compute the warping function. Using the corresponding lattice points between $L$ and $L_{r}$ as control points, this algorithm generates a warping function $\mathbf{W}: \mathbb{R}^{2} \longmapsto \mathbb{R}^{2}$, which is a bijective pixel-wise mapping from the input texture $\mathcal{P}$ to the straightened out texture $\mathcal{P}_{I}$. A geometric deformation field $d_{g e o}(x, y)$ is then computed from the warping function by converting the global position mapping to a local displacement vector, i.e., $d_{g e o}(x, y)=\mathbf{W}(x, y)-[x, y]$. 


\subsubsection{Lighting Deformation Field Extraction}

To model a type III NRT, we need to extract the lighting effects on the texture. The lighting deformation field extraction algorithm works as follows: (1) straighten a Type III near-regular texture using the geometric deformation field extraction described in the previous section; (2) apply Tsin et al. [Tsin et al., 2001] algorithm to extract the lighting map on the plane; (3) apply the inverse geometric deformation field to map the lighting map back to the original texture.

The method described above works well for textures placed on surfaces with moderate bends; however, without knowing the surface geometry, straightening the lattice alone does not truly flatten a sharply folded textured surface. The shape and size of some tiles may differ significantly at the regions where geometric distortions are severe, e.g., sharply folded cloth ridges. These residual geometric distortions need to be further removed, otherwise they will be mistakenly identified as part of the lighting effects. We use an affine local-registration algorithm to correct the residual distortions between each tile and a reference tile. We also apply a bilateral filtering [Tomasi and Manduchi, 1998] to the light deformation field to further reduce falsely detected lighting effects. Figure 2.3(d) shows the result of a lighting deformation field extracted from a piece of cloth with sufficient amount of folding.

\subsubsection{Texture Regularity Measurement}

Based on the concept of departures from regularity, we can further quantitatively measure the degrees of regularity of an NRT. Using the same symbolic and geometric convention for a lattice structure as in Equation (2.1) of Section 2.2.1, we define a pair of quantitative measurements for near-regular textures. This pair of statistical measures characterizes the regularity of a near-regular texture from two different perspectives, $G$ for geometric deformation and $A$ for appearance of color intensity variation, with the understanding that the color variation can be caused by both inherent tile color differences and external lighting changes.

Geometric regularity- $\mathrm{G}$ score

$G=\sum_{i=1}^{N_{i}} \frac{\left(l_{i}-\left\|\mathbf{t}_{\mathbf{1}}\right\|\right)^{2}}{\left\|\mathbf{t}_{\mathbf{1}}\right\|^{2}}+\sum_{j=1}^{N_{j}} \frac{\left(l_{j}-\left\|\mathbf{t}_{\mathbf{2}}\right\|\right)^{2}}{\left\|\mathbf{t}_{\mathbf{2}}\right\|^{2}}+\sum_{k=1}^{N_{k}} \frac{\left(l_{k}-\left\|\mathbf{t}_{\mathbf{1}}+\mathbf{t}_{\mathbf{2}}\right\|\right)^{2}}{\left\|\mathbf{t}_{\mathbf{1}}+\mathbf{t}_{\mathbf{2}}\right\|^{2}}+\sum_{m=1}^{N_{m}} \frac{\left(l_{m}-\left\|\mathbf{t}_{\mathbf{1}}-\mathbf{t}_{\mathbf{2}}\right\|\right)^{2}}{\left\|\mathbf{t}_{\mathbf{1}}-\mathbf{t}_{\mathbf{2}}\right\|^{2}}$ 


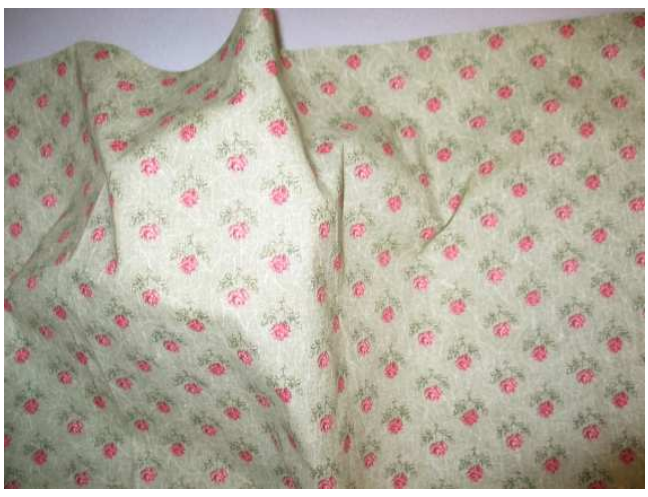

(a)

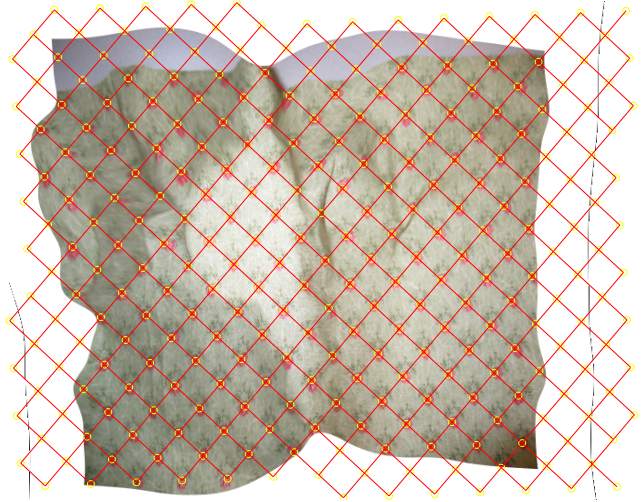

(c)

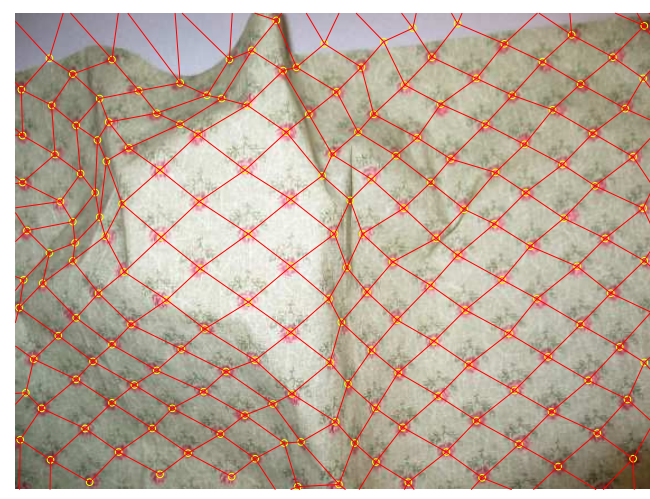

(b)

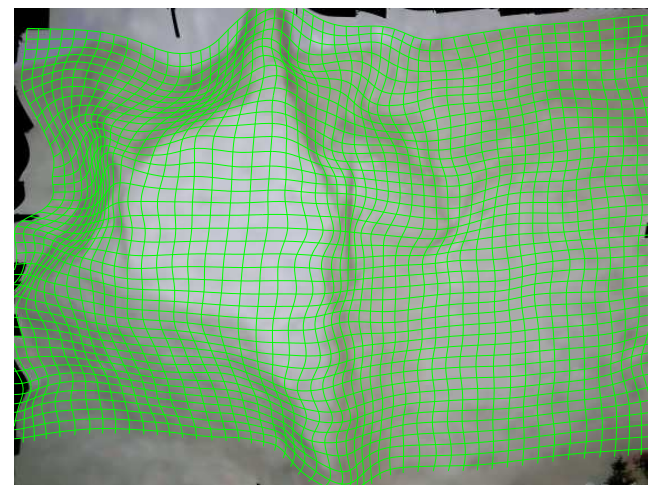

(d)

Figure 2.3. (a) input texture (b) input texture with a user-identified lattice (c) straightened texture $(\mathrm{d})$ extracted geometric deformation field (green mesh) and lighting deformation field [Liu et al., 2004].

\section{Appearance regularity-A score}

$$
A=\frac{1}{m} \sum_{i=1}^{m} \operatorname{std}\left(\left[T_{1}(i), T_{2}(i), \ldots, T_{n}(i)\right]\right)
$$

where $T_{j}$ is a column vector consisting of all pixels of tile $t$ of a regular texture; $m$ is the number of pixels within a tile. The standard deviation is computed over all tiles. We also apply a gaussian filtering to all tiles so that the color/intensity variation due to the misaligned pixels would not bias the measurement.

Using the GA-scores, we can more precisely define different types of textures (Table 2.1). Strictly speaking, a texture is regular or Type 0 if $G=A=0$; Type I near-regular if $G=0$ and $A>0$; Type II if $G>0$ and $A=0$; and Type III if $G>0$ and $A>0$. In reality, it is difficult to find clear boundaries between different types of textures as these textures form a continuous texture spectrum (Figure 1.1). However, these measurements 


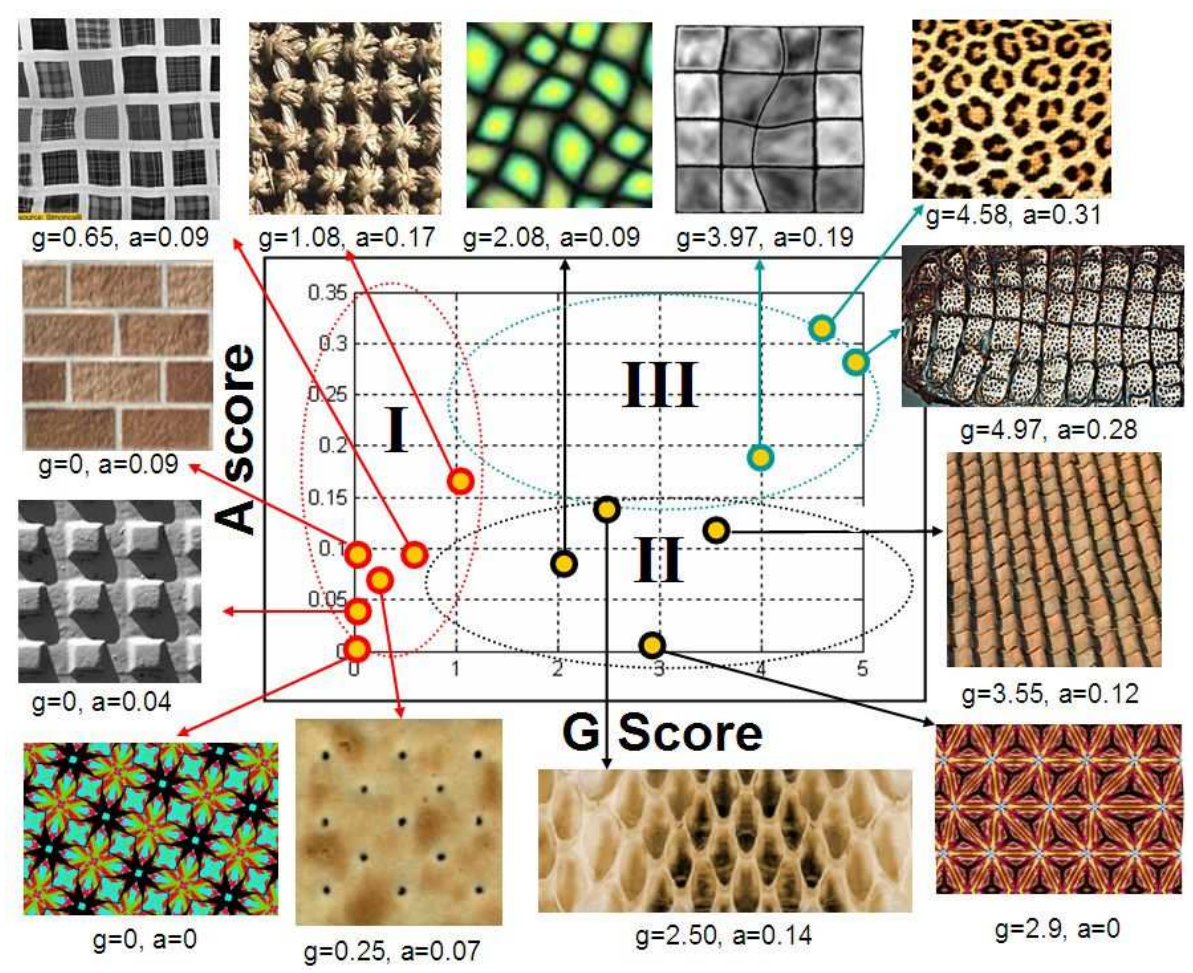

Figure 2.4. Textures plotted using our geometric $(\mathrm{G})$ and appearance (A) regularity measures. The higher the scores, the more irregular the texture. Notional classification regions for Type I,II and III near-regular textures are indicated [Liu et al., 2004].

provide a quantitative guideline for texture classification by regularity (Figure 2.4) and for texture synthesis results evaluation (section 2.5, Figure B.2-B.14).

\subsection{NRT Synthesis}

After NRT analysis, an NRT is characterized by a Type I texture and a pair of geometric and lighting deformation fields. We can synthesize a near-regular texture by synthesizing these three components, then apply the synthesized deformation fields back to the synthesized Type I texture to generate the output texture. Our texture synthesis algorithm consists of three modules: Type I texture synthesis, geometric deformation field synthesis and lighting deformation field synthesis. By combining these three modules, we can synthesize all types of NRTs.

\subsubsection{Type I NRT Synthesis}

The type I NRT synthesis algorithm [Liu and Tsin, 2002; Liu et al., 2005] synthesizes textures by pasting tiles based on the lattice structure of an input NRT. These tiles are 

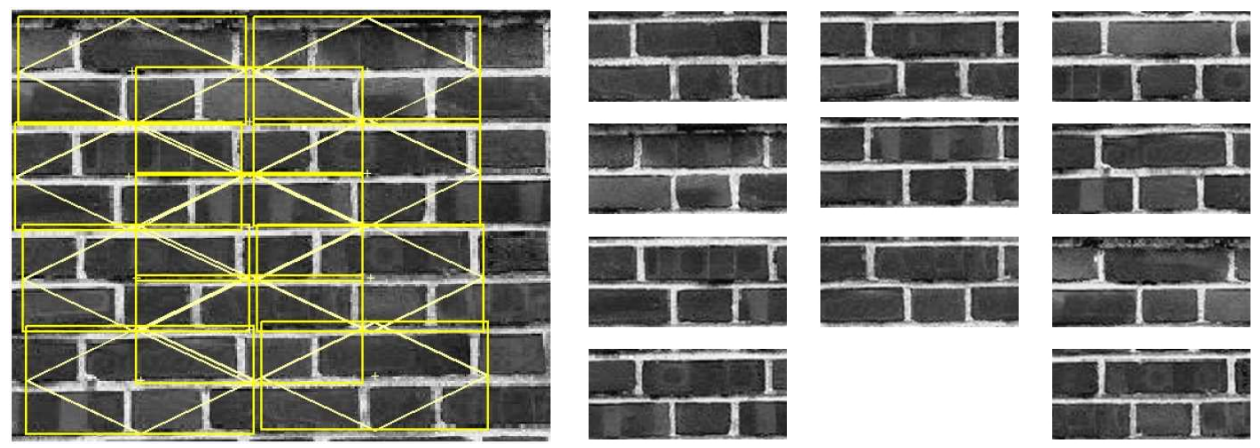

Figure 2.5. (a) Illustration of maximum tiles (yellow rectangles) (b) The maximum tile set extracted based on lattice in (a) [Liu and Tsin, 2002].

defined by the lattice of the input NRT. A tile represents the smallest parallelogramshaped region on a regular texture that can reproduce the texture patterns under the texture's translation subgroup. For regular texture, only one tile is needed for synthesizing the same texture. For near-regular texture, we need to collect a set of sample tiles with roughly the same size and shape but varying color and intensity (Figure 2.5). The output texture is then synthesized by randomly picking a tile from the tile set and pasting the tile to the synthesizing image with overlapping in scan-line order. We summarize the Type I NRT synthesis algorithm below:

1. Tile extraction: Maximum tiles $T_{i}$ 's are extracted from an input NRT where a maximum tile is the rectangular region that circumscribes a parallelogram in the lattice of the input NRT. A maximum tile set $\mathcal{T}$ is the set that contains all maximum tiles of the NRT. Besides $\mathcal{T}$, another maximum tile set $\mathcal{T}_{h}$ is constructed by including those maximum tiles centered on the lattice points. Figure 2.5 shows the definition of maximum tile and maximum tile set.

2. Tile placement: Given the image size of a texture to be synthesized, a new lattice that covers the image region is generated using the generating vectors $\left(\mathbf{t}_{1}\right.$ and $t_{2}$ ) of the lattice found in the input texture. In the synthesis process, tiles are initially placed one by one at the lattice points and half-way lattice points of the new lattice, where a half-way lattice point is the center of a parallelogram in the lattice.

3. Tile selection: At each lattice or half-way lattice points, alternatively select the tile set $\mathcal{T}$ or $\mathcal{T}_{h}$. For each tile in the selected tile set, we compute its color difference to the existing synthesized image in the overlapped region. A candidate 
tile set is formed by selecting those tiles that have color difference less than a threshold. A tile is then randomly picked from the candidate tile set. If the candidate tile set is empty, the tile with minimum difference is picked. The size of the candidate tile set varies at each lattice point.

4. Local adjustment: The selected candidate tile is registered to the existing synthesized image using a correlation-based method. This adjusts the placement location of the tile around a lattice point.

5. Tile Stitching: Dynamic programming is used to "stich" together the overlapping tiles in a similar manner as described in [Efros and Freeman, 2001]. Additionally, blending is applied to smooth seams generated by stitching.

\subsubsection{Type II NRT Synthesis}

Type II NRTs are synthesized by combining the synthesis of the type I NRT and the geometric DF [Liu and Lin, 2003]. From section 2.2.1, we obtain a mesh-like bijective mapping representing the deformation field $d_{\text {geo }}$ of the input near-regular texture $\mathcal{P}$ to its nearest geometrically regular texture $\mathcal{P}_{I}$, where $d_{\text {geo }}\left(\mathcal{P}_{I}\right)=\mathcal{P}$ (Figure 2.6). Utilizing the deformation field duality, we can visualize the geometric deformation field $d_{g e o}$ in the hue, saturation, and value (HSV) color space. The hue and saturation components represent the direction angle and the length of a 2D displacement vector, respectively, and the value component is set to 1 . Under this representation, a pure white color means zero movement, and a red color means a movement in the positive $x$ direction (Figure 2.6).

Geometric deformation fields of near-regular textures are usually non-regular, stochastic textures. Ideally, we can apply any existing texture synthesis algorithm to generate a new deformation field of any size; however, the smoothness of the synthesized field is more demanding because an artifact edge in the synthesized field means a rapid change of the displacements of the pixels, which usually results in a discontinuous warping function. Therefore, the smoothness of the synthesized deformation field is required in the deformation field synthesis process.

Figure 2.6 illustrates our algorithm for Type II texture synthesis, which includes Type I texture synthesis algorithm and the geometric deformation field synthesis algorithm. Since the geometric deformation field is computed by the MFFD algorithm [Lee 


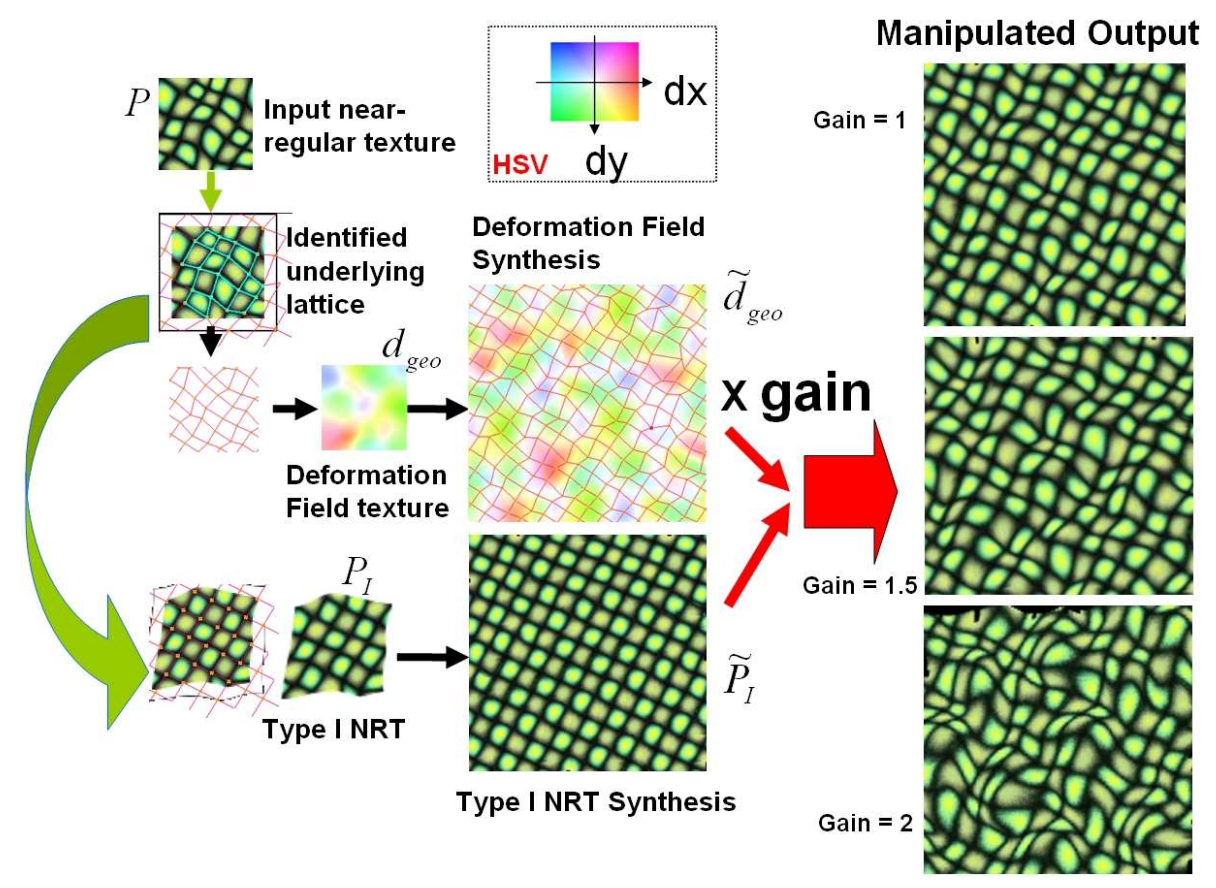

Figure 2.6. An overview of the geometric deformation field synthesis [Liu and Lin, 2003] and manipulation algorithm. Starting with an input near-regular texture (NRT) $\mathcal{P}$, its geometrically regular version $\mathcal{P}_{I}$ is obtained by "straightening out" its underlying lattice $L$ into the nearest regular lattice such that $L=d_{\text {geo }}\left(L_{r}\right)$ and $\mathcal{P}=d_{\text {geo }}\left(\mathcal{P}_{I}\right) . \mathcal{P}_{I}$ is a Type I NRT by definition and $d_{\text {geo }}$ a geometric deformation field treated as a texture, is usually not a regular or near-regular texture. We synthesize $\mathcal{P}_{I}$ to $\widetilde{\mathcal{P}}_{I}$ using a Type I NRT synthesis algorithm developed in [Liu et al., 2005], and synthesize the deformation field $d_{\text {geo }}$ to $\widetilde{\mathcal{D}}_{\text {geo }}$ based on the algorithm proposed in [Efros and Leung, 1999]. Finally, the NRT manipulation is achieved by applying different gains to $\widetilde{\mathcal{D}}_{\text {geo }}$. When gain $=1$, the output texture is most similar to the input texture [Liu et al., 2004].

et al., 1995] based on the correspondence of the lattice $L$ of an input texture and its regular counterpart, $L_{r}$, we just need to synthesize a lattice when synthesizing a geometric deformation field. We use a pixel-based synthesis algorithm [Efros and Leung, 1999] to synthesize the displacements of lattice points. These displacement vectors are defined between the corresponding lattice points of the input (deformed) lattice $L$ and its regular counterpart $L_{r}$. A deformed lattice is then constructed based on the synthesized displacements, and the MFFD algorithm is used to generate a pixel-wise deformation field based on the synthesized deformed lattice. The smoothness of the geometric deformation field is guaranteed by the MFFD algorithm. 
Figure 2.7 shows some of our synthesis results of type II NRTs. The second column show the synthesized geometric DFs using the HSV color space. The red color corresponds to a rightward displacement while cyan and white color represent leftward and zero displacement respectively (see the HSV subplot in Figure 2.6). The geometric DFs in the top row are nice examples for understanding the correspondence between a deformation field and its HSV representation. It also illustrates the textural and functional properties of the deformation field. Figure 2.8 shows a quantitative comparison result of a checkerboard-like yellow-green pattern of our synthesis algorithm against four other texture-synthesis algorithms [Efros and Leung, 1999; Efros and Freeman, 2001; Wei and Levoy, 2000; Kwatra et al., 2003]. One can observe (Figure 2.8(a)-(f)) that only our algorithm preserves the color alterations in the input texture sample. In the $2 \mathrm{D}$ regularity space (geometry versus color variations) defined in Section 2.2.3, our synthesis result is the closest to the input texture (Figure 2.8(g)).

\subsubsection{Type III NRT Synthesis}

The crucial component in a successful type III NRT synthesis is the lighting DF synthesis, which can be combined with type NRT I NRT synthesis and geometric DF synthesis. For an NRT placed in a realistic environment, its geometric DF and lighting DF are strongly associated. To achieve realistic NRT synthesis, we need to synthesize this pair of deformation fields simultaneously. Based on our duality view of the deformation field and the strong relation between the geometric and lighting DFs, the type III NRT synthesis can be formulated as an image analogy problem [Hertzmann et al., 2001]. We call this deformation field analogy.

Figure 2.9 shows this formulation, where $A=d_{\text {geo }}$ and $A^{\prime}=d_{\text {light }}$ are the geometric and lighting deformation fields respectively. $B=\widetilde{d}_{g e o}$ is a synthesized geometric deformation field. With this formulation, the image analogy algorithm [Hertzmann et al., 2001] can be used to infer the lighting deformation field, $B^{\prime}=\widetilde{d}_{\text {light }}$. There are two ways to approach this problem. We can either (1) synthesize $A$ to $B$ then look for $B^{\prime}$ using analogy, or (2) synthesize $A^{\prime}$ to $B^{\prime}$ then look for $B$ using analogy (Figure 2.9). Theoretically, following either route we should be able to achieve a new geometry/lighting deformation field pair as the result of deformation field analogy. Based 

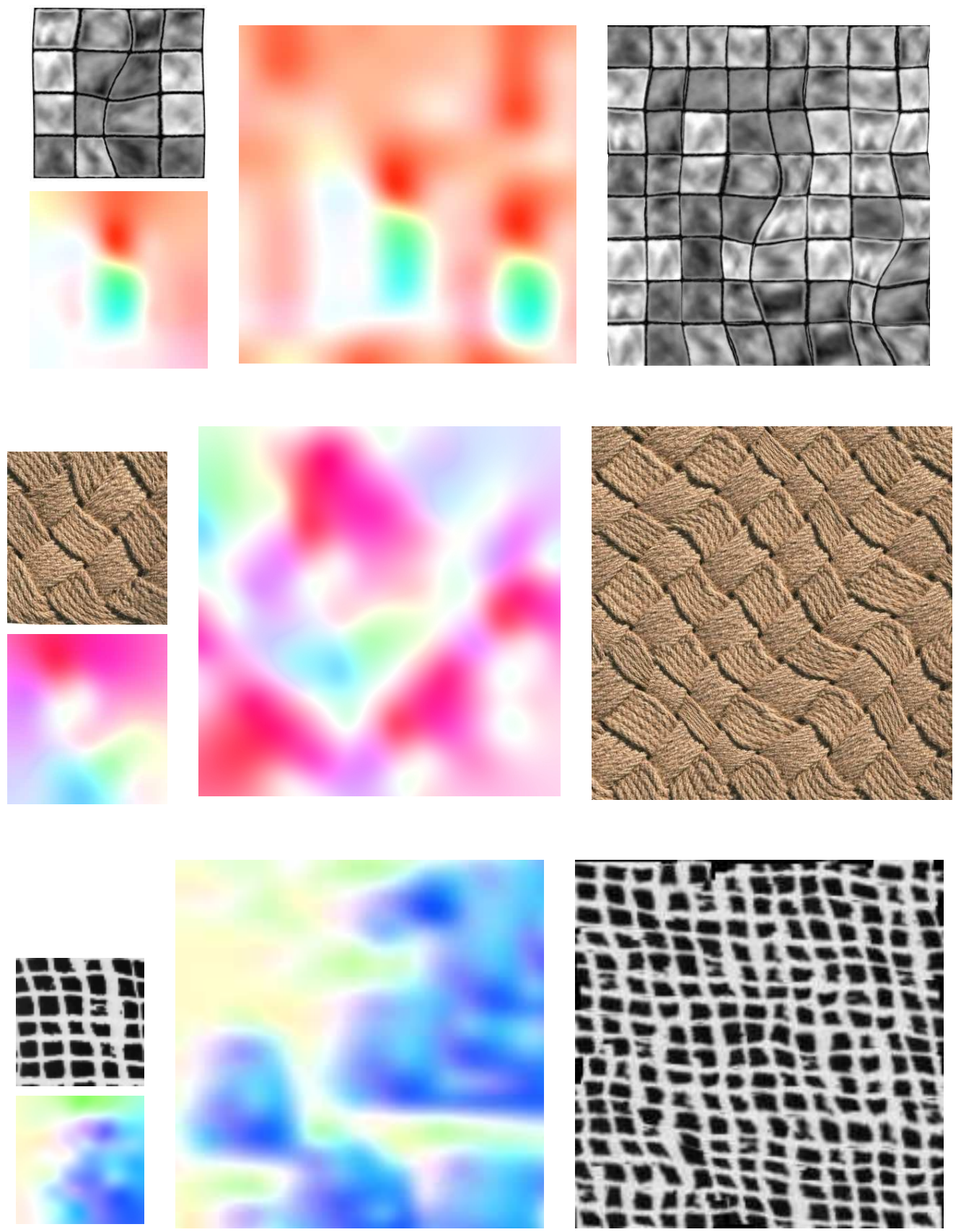

Figure 2.7. Type II NRT synthesis results. Left: input texture and extracted geometric deformation field. Center: synthesized geometric deformation field. Right: output texture [Liu and Lin, 2003].

on our experiments, the first route is more favorable: we apply the geometric deformation field synthesis algorithm to generate a new geometric DF $\widetilde{d}_{g e o}$ first, then apply image analogies to synthesize the lighting deformation field corresponding to $\widetilde{d}_{g e o}$. The 
geometric deformation field provides the necessary causal constraints on the lighting deformation field. See Figure 2.10 for an example of deformation field analogies.

\subsection{NRT Manipulation}

One novel and insightful realization of NRT computational framework is the duality of deformation fields. On the one hand, a deformation field is a multi-modal, multidimensional mapping that warps a regular texture into its near-regular form or vice versa. On the other hand, a deformation field itself is a texture that can be subjected to texture synthesis and manipulation as demonstrated in this thesis. Our approach to manipulation of near-regular textures is achieved through manipulation of their deformation fields.

\subsubsection{Texture Regularity Manipulation}

Beyond faithfully synthesizing an NRT, we can also manipulate the geometric regularity of the synthesized texture. The synthesized deformation field $\widetilde{\mathcal{D}}_{\text {geo }}$ provides a parameterized approximation of geometric regularity similar to the input sample texture. We can multiply the pixel-wise magnitude of the deformation field by a gain factor: when gain $=1$ the deformation field is faithful to the input; when $\mid$ gain $\mid<1$ or $\mid$ gain $\mid>1$ the magnitude of the deformation field departs from the input texture's regularity. Figure 2.11 shows several synthesized texture of which the regularity is manipulated. One can observe the consistency between the gain factor, the regularity measures, and the visual effects in the texture manipulation results.

\subsubsection{Texture Replacement in Real Photos}

The goal of texture replacement in a single real-world photo is to preserve the original visual effects of geometric deformation and photometric variation in an image but with a different texture given only a single image. For a near-regular texture in an image, we can achieve this goal by extracting geometric and lighting deformation fields from the near-regular texture and apply the deformation fields to a new texture $\mathcal{P}$, regardless of whether $\mathcal{P}$ is regular or not. Figures 2.12 and 2.13 show the results from our texture replacement algorithm.

Figure 2.14 shows another texture replacement example from an outdoor photo. Figure 2.15(a) explains how the underlying lattice of the outdoor scene is extracted. 
This building photo, as a texture, has only three large tiles (each is three-floors high). These are tiles with curved boundaries, captured by the user interface using cubic spline interpolation. The control points can be adjusted by the user for a better fit. The lighting deformation field of this texture is shown in Figure 2.15(b).

There many potential applications of this type of manipulation, especially in image editing, e.g., architecture and interior design, fashion design preview. In Chapter 4, we demonstrate novel applications in video texture replacement, such as changing the fabric texture on moving cloth or superimposing text on a dress. 


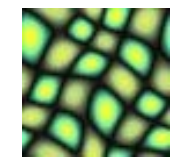

(a) input sample $\mathrm{G}=2.1, \mathrm{~A}=0.09$

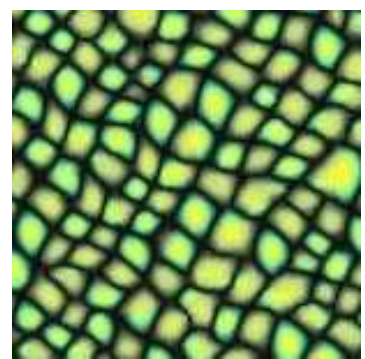

(d) Wei and Levoy [2000] $\mathrm{G}=3.8, \mathrm{~A}=0.22$

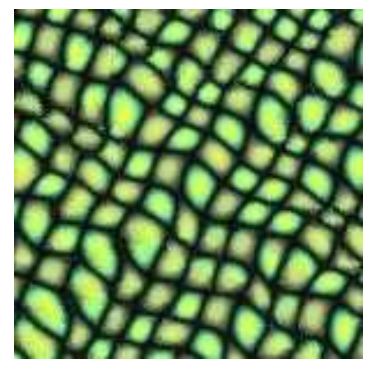

(b) Efros and Leung [1999] $\mathrm{G}=3.9, \mathrm{~A}=0.16$

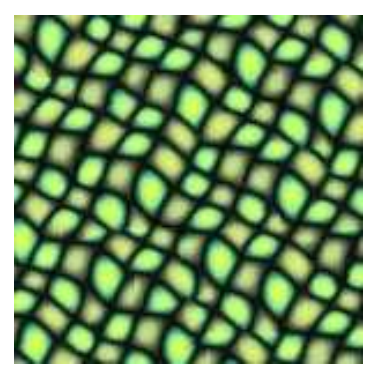

(e) Kwatra et al. [2003] $\mathrm{G}=1.7, \mathrm{~A}=0.15$

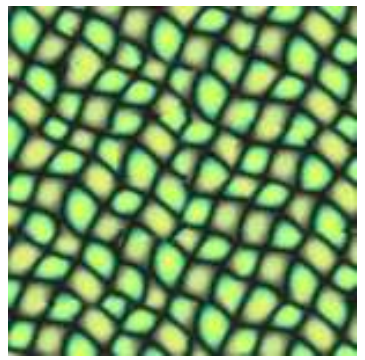

(c) Efros and Freeman [2001] $\mathrm{G}=1.5, \mathrm{~A}=0.14$

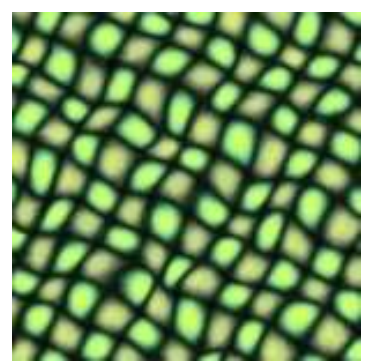

(f) Our method

$\mathrm{G}=1.8, \mathrm{~A}=0.11$

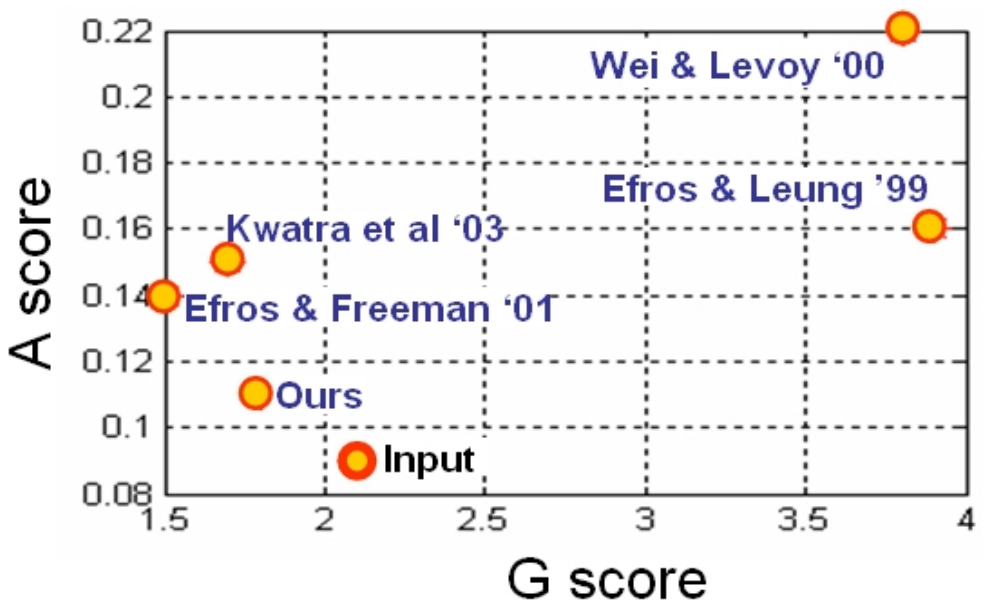

(g) GA-scores plot of synthesized textures

Figure 2.8. One of our texture-synthesis results compared with other methods on a type II NRT. One can observe on close inspection the regular alterations in color (... yellow, green, yellow, green, ...) of the input texture. This regularity is more faithfully preserved by our texture synthesis method (Figure 2.6, when gain $=1$ ). In the quantified geometry-appearance regularity space represented by the $G$ - $A$ regularity scores (Section 2.2 .3 ), our output is the closest to the input texture [Liu et al., 2004]. 


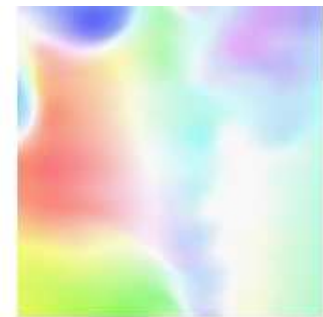

A

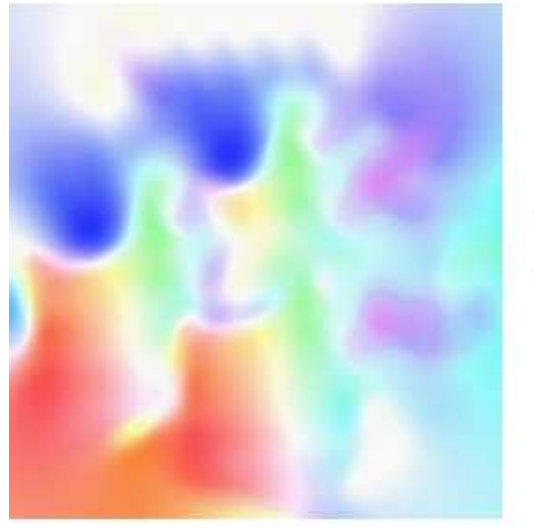

B

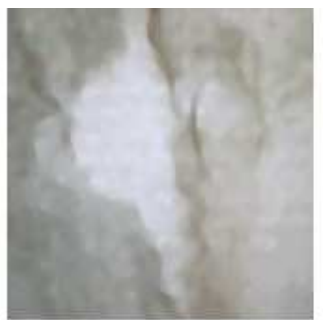

A'

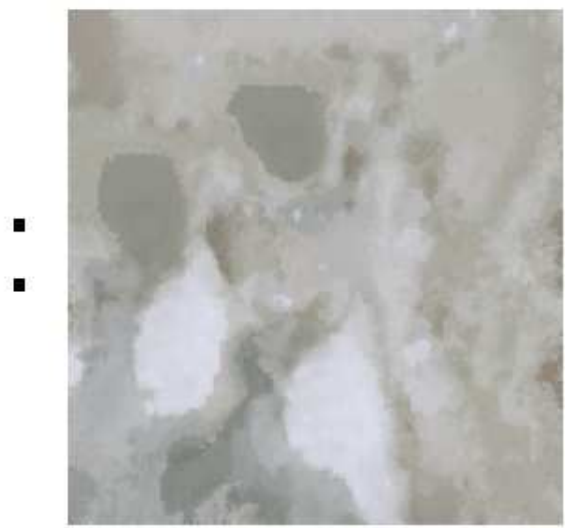

B'

Figure 2.9. Formulation and result of deformation field analogy, where $A$ and $A^{\prime}$ are the geometry and lighting deformation fields respectively. $B$ is a synthesized geometric deformation field generated by the method in section 2.3.2. $B^{\prime}$ is an analogy result generated by image analogy [Hertzmann et al., 2001]. 

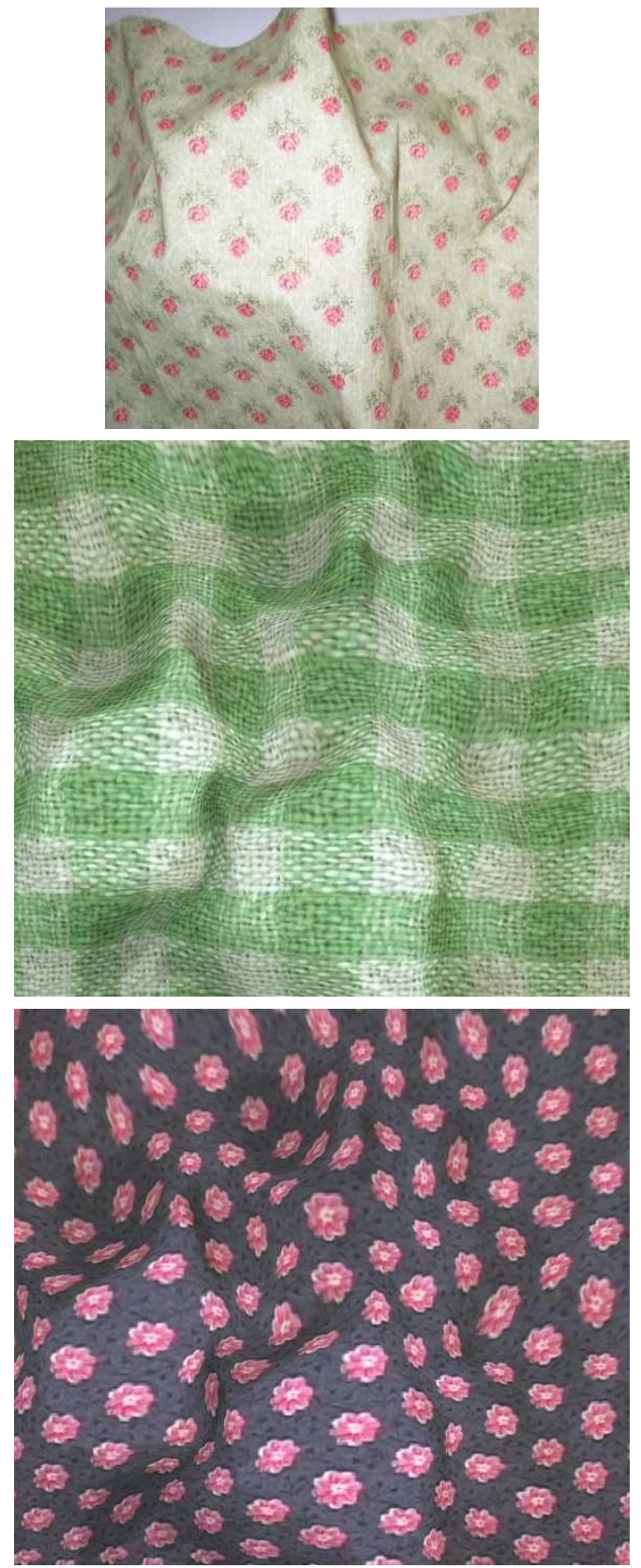
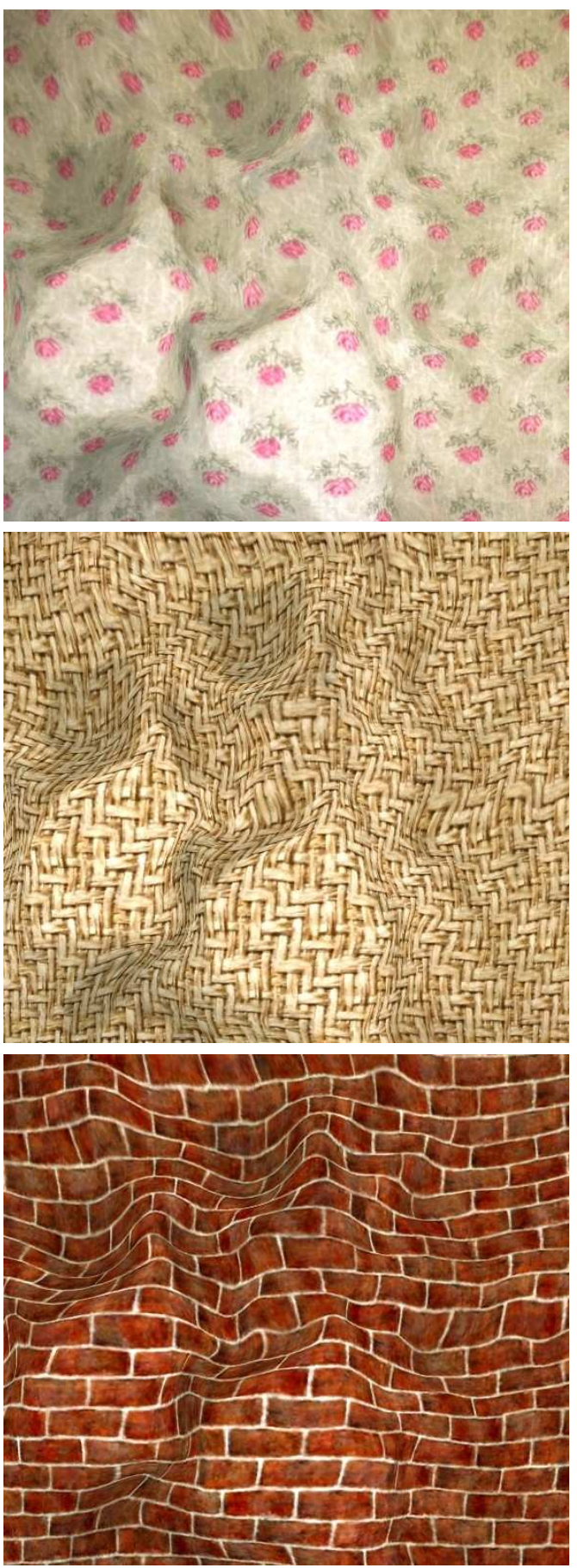

Figure 2.10. Type III near-regular texture synthesis results. Top left is the input texture. The geometric and lighting deformation fields of the input and synthesized textures are shown in Figure 2.9. 


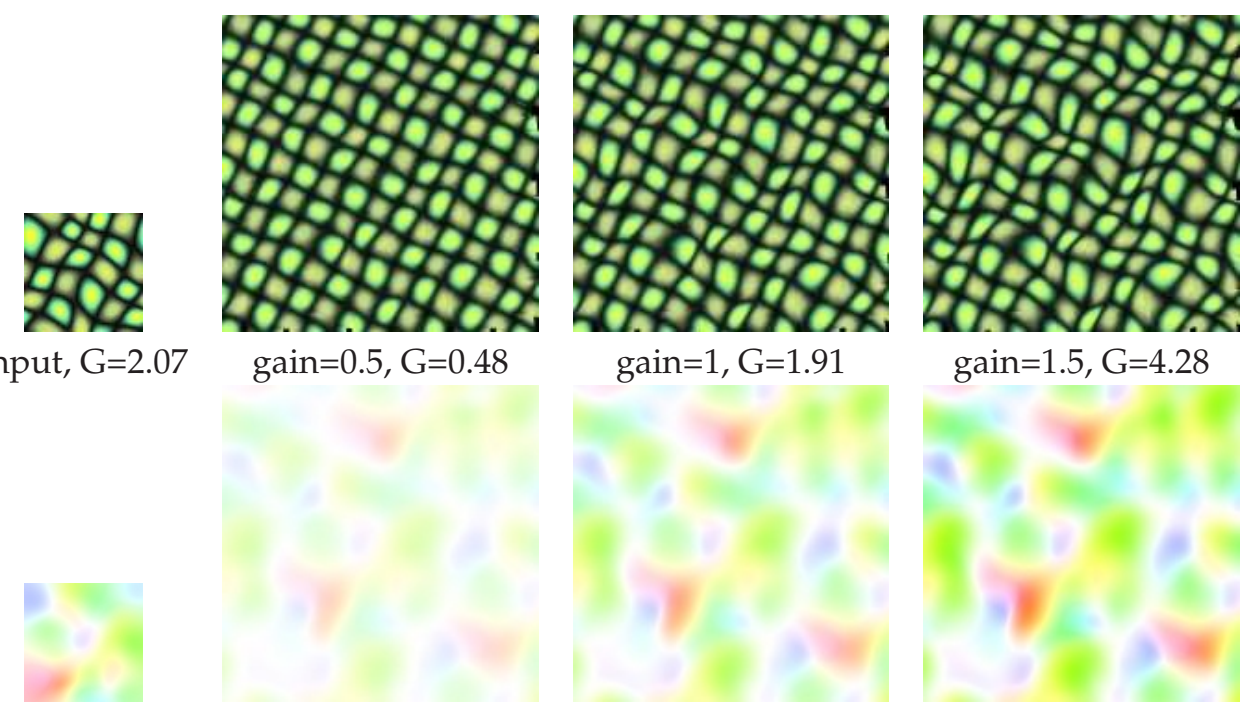

Figure 2.11. Results of manipulating the geometric regularity, where the magnitude of all vectors in the deformation field is uniformly scaled by the gain parameter, $\mathrm{G}$ is the geometric regularity of the texture. The bottom row shows the corresponding geometric fields.
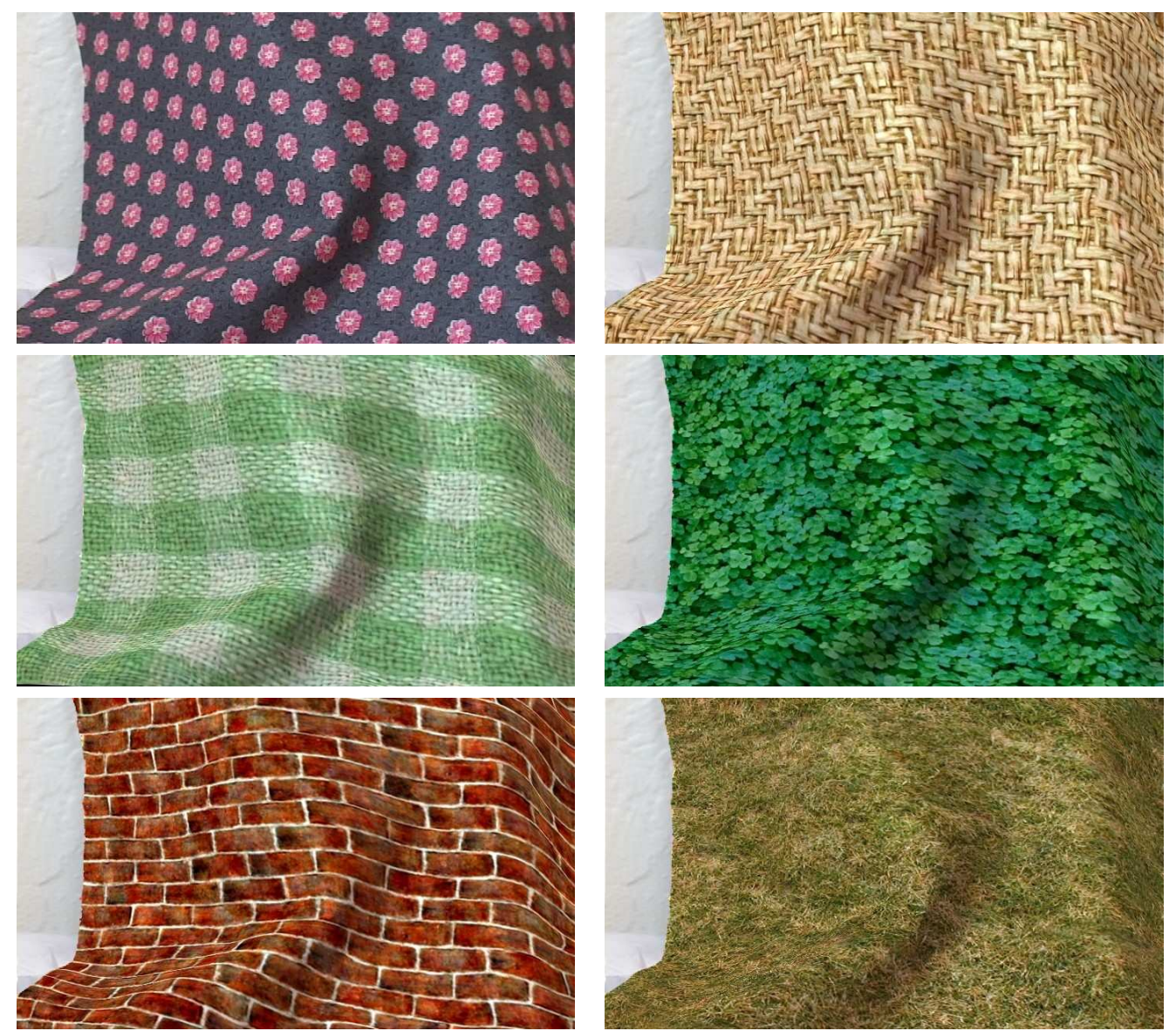

Figure 2.12. Texture replacement of a fabric texture on cloth (top left is the input photo) 

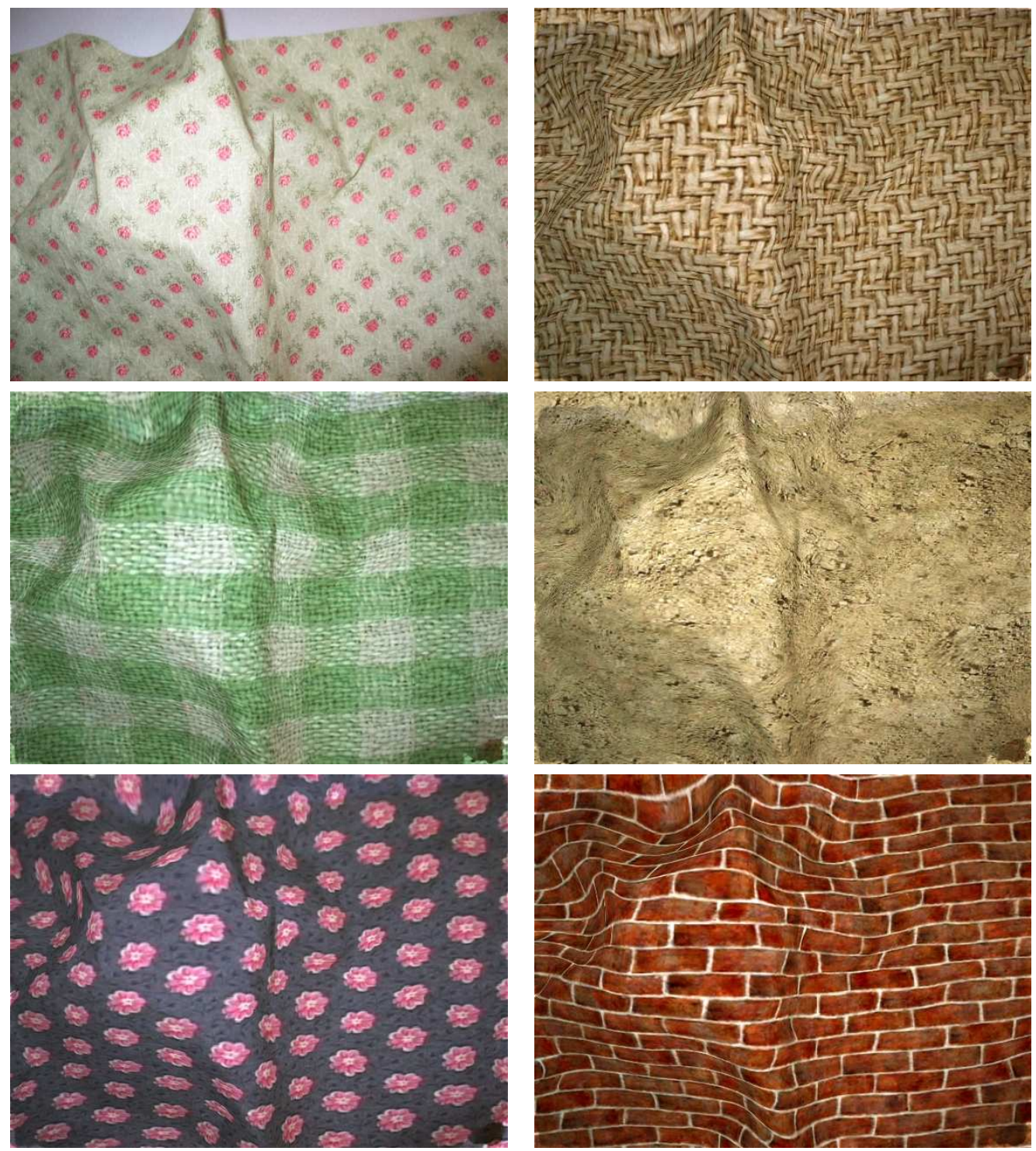

Figure 2.13. Texture replacement of a rose patterned cloth (top left is the input photo). The rest are texture replacement results of our method. Our method takes one input image and a target texture sample. If the target texture is nearregular, it is first synthesized using Type I near-regular texture synthesis before the deformation fields are applied. Deformation fields of the input image are shown in Figure 2.3. 

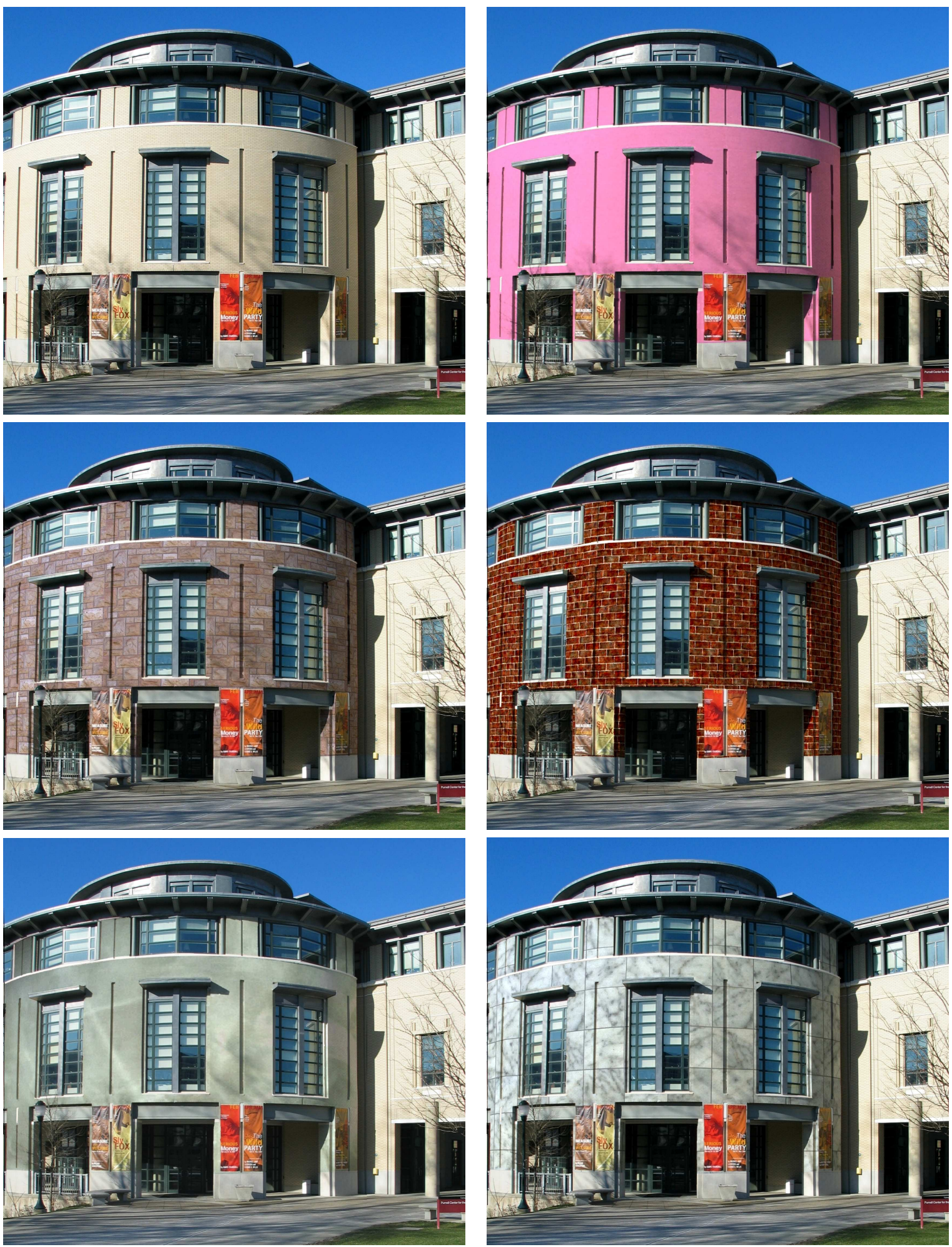

Figure 2.14. Texture replacement on an outdoor photo(top left). The lighting deformation field of the input texture is shown in Figure 2.15(b) 


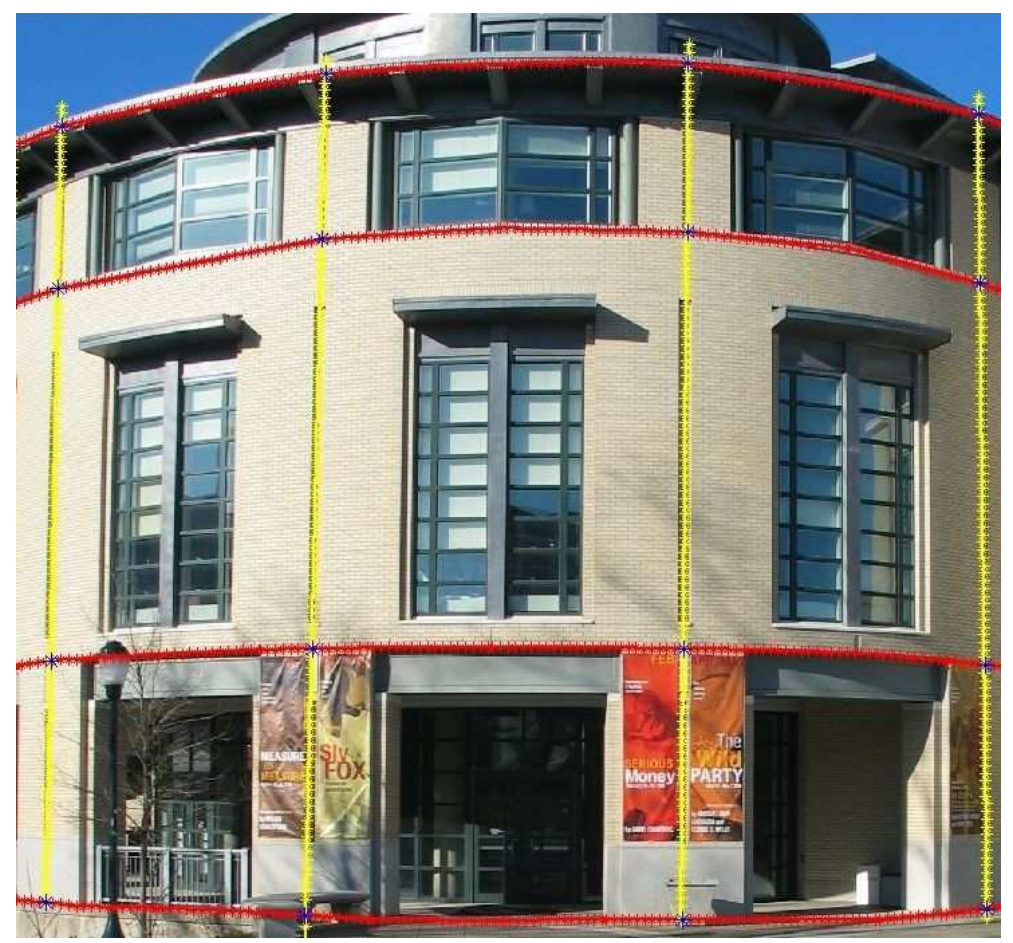

(a)

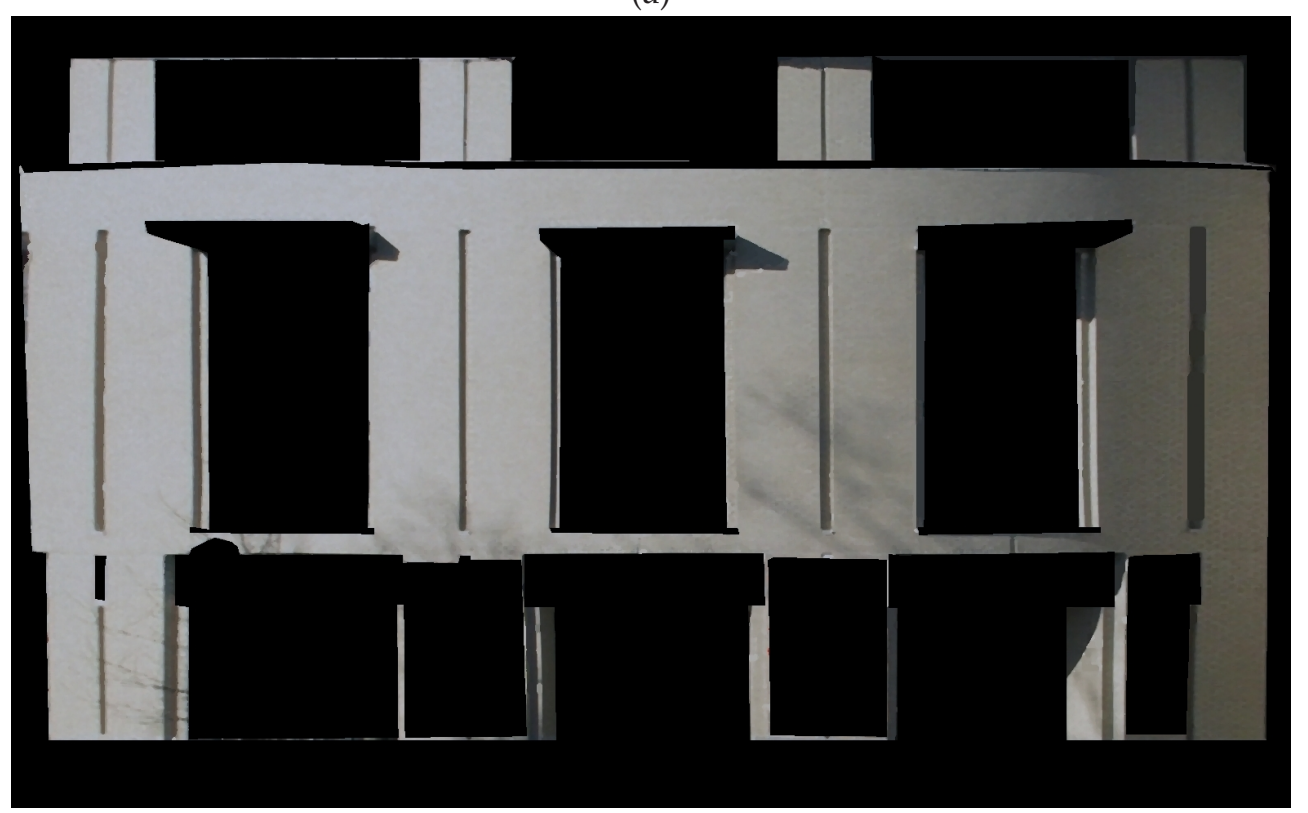

(b)

Figure 2.15. (a) Three complete vertical tiles of an NRT, each tile is three-floors high, are identified in an outdoor photo. Internal correspondences are added (each large tile is divided into three portions) for an extra assurance on global as well as local geometric consistency during deformation. Less than 4 minutes are needed for the lattice extraction process, initiated by the user (Section 2.2.1) [Liu et al., 2004]. (b) Lighting deformation field. 


\subsection{Texture Synthesis Algorithm Comparison for NRT Synthesis}

To evaluate the performance of existing texture synthesis algorithms on NRTs, we compare our algorithm and four other synthesis algorithms on 43 NRTs $^{1}$. Conventionally, the performance of a texture synthesis algorithm is judged by examining the results visually, which could be subjective and inconsistent among different viewers. To better evaluate the synthesis results, we employed both an objective criterion and a user study test.

The regularity preservation test utilizes the texture regularity of NRT defined in section 2.2.3 to evaluate the synthesized textures. A simple screening criterion is used to determine whether an NRT is faithfully synthesized or not by first checking if the lattice structure is faithfully maintained. Furthermore, we can quantitatively evaluate the synthesis results by computing the ga-score difference between the input and synthesized textures; we performed the user evaluation test by asking 10 human subjects to grade the results of different synthesis algorithms based on a set of 4-scale scores predefined.

\subsubsection{A Brief Review of Selected Algorithms}

In this comparison study, we consider the image-based synthesis algorithms because we are interested in regular and near-regular texture synthesis. In particular, we compare our synthesis algorithm with the graph cut approach [Kwatra et al., 2003], the patch-based approach [Liang et al., 2001b,a], and the regularized patch-based approach [Lin et al., 2004]. We briefly describe these algorithms and analyze their treatments on regular and near-regular texture synthesis.

Graph Cut Texture Synthesis. Kwatra et al. [2003] demonstrate a very effective general texture synthesis algorithm. Texture is synthesized by overlaying the entire input texture onto the synthetic texture at various offsets and using a graph cut algorithm to find the optimal region to add to the synthetic texture. The graph cut algorithm avoids the need for a fixed, a-priori patch size and scales well to any dimension (such as video). However, for near regular textures the choice of offsets is as important as finding low-error seams. If the input texture is copied onto the synthesized texture at

\footnotetext{
${ }^{1}$ An NRT databse website, http://graphics.cs.cmu.edu/data/texturedb/gallery/albums.php, has been created at CMU.
} 
an offset that is inconsistent with the periodicity of the texture, any selection of seams will still violate the global regularity of the texture. Kwatra et al. describe patch placement algorithms which do a fair job of finding low error offsets. The error is defined as the sum of squared difference (SSD) between the pixels in the overlapping region of the input texture and the texture being synthesized. They treat the input texture as a template and compute the correlation between the template and the texture being synthesized to find the low error offsets. The maximum correlation offsets often, but not always, correspond to the offsets preserving the periodicity of the input texture.

Regularized Patch-based Texture Synthesis. The regularized patch-based texture synthesis algorithm is modified from the patch-based texture synthesis algorithm in order to deal with NRTs in which each texture element may not be well circumscribed by a parallelogram. The algorithm allows the parallelograms on a regular lattice to be deformed to quadrilaterals so that the texture elements can be separated by the deformed lattice. In other words, a geometrically-irregular NRT is deformed to a geometrically-regular NRT. A modified patch-based approach is then applied to synthesize the geometrically-regular texture. The modification to the patch-based approach allows patches to be pasted along the lattice axis direction and allows the patch shape to be a parallelogram rather than a rectangle. The patch-size and lattice construction vectors are provided by a user who identifies the underlying lattice structure of the input NRT. A synthesized inverse deformation is used to warp the synthesized regular texture to an NRT. The details of the algorithm is described in [Lin et al., 2004].

Table 2.2 is a summary for these algorithms. We compare the patch shape/size determination, patch extraction, patch placement, and patch stitching methods used in these algorithms, where a patch is a 2D sample of neighboring pixels extracted from the input texture. Patch shape/size and extraction refer to how the shape and size of the region are determined, and where each patch is located in the input texture. Patch placement and stitching refer to how the patches are placed and stitched in the synthesized texture.

Patch-based Texture Synthesis. Liang et al. [2001b; 2001a] develop a patchbased synthesis algorithm. The basic idea of the algorithm is to synthesize textures by directly copying image patches from the input texture. The major difference from 
Table 2.2. Summary of four synthesis algorithms. A patch is a $2 \mathrm{D}$ sample of neighboring pixels extracted from the input texture image. Patch shape/size and extraction refer to how the shape and size of the region are determined, and where each patch is located in the input texture. Patch placement and stitching refer to how the patches are placed and stitched in the synthesized texture.

\begin{tabular}{|l|l|l|l|l|}
\hline Algorithms & Graph cut & $\begin{array}{l}\text { Near-regular } \\
\text { synthesis }\end{array}$ & $\begin{array}{l}\text { Regularized } \\
\text { patch-based }\end{array}$ & Patch-based \\
\hline $\begin{array}{l}\text { Patch } \\
\text { shape/size }\end{array}$ & $\begin{array}{l}\text { partial or whole } \\
\text { input texture image }\end{array}$ & $\begin{array}{l}\text { translational symmetry } \\
\text { analysis, user intervention }\end{array}$ & $\begin{array}{l}\text { user identified lattice, } \\
\text { quadrilateral patch } \\
\text { lattice points }\end{array}$ & $\begin{array}{l}\text { user defined, } \\
\text { rectangular patch }\end{array}$ \\
\hline $\begin{array}{l}\text { Patch } \\
\text { extraction }\end{array}$ & random locations & lattice points & lattice points & patch grids \\
\hline $\begin{array}{l}\text { Patch } \\
\text { placement }\end{array}$ & $\begin{array}{l}\text { random or maximal } \\
\text { correlation locations }\end{array}$ & lattice points \\
\hline $\begin{array}{l}\text { Patch } \\
\text { stitching }\end{array}$ & $\begin{array}{l}\text { graph cut \& } \\
\text { blending }\end{array}$ & $\begin{array}{l}\text { dynamic programming } \\
\text { \& blending }\end{array}$ & image-feathering & image-feathering \\
\hline
\end{tabular}

other image-based approaches is that they apply a modified approximate nearest neighbor technique to speed up the search for the best matched patch. With this improved search speed, the algorithm can run in real-time and reach similar image quality as other image-based synthesis algorithms. The image feathering technique is used in the patchbased synthesis approach to blend the overlapping regions of patches. This might blur the overlapping region slightly compared to the dynamic programming technique used in NRT synthesis or the graph cut technique in the graph cut synthesis approach.

Patch placement in the patch-based approach is very different from that in NRT synthesis. In the patch-based approach, the patch is rectangular and the patches are pasted in a scan-line order. Since the patch size and placement offset are arbitrarily defined by a user, they may not match the lattice structure of the input NRT.

\subsubsection{Analysis of Comparison Results}

We compared the synthesis results of these four algorithms on regular textures (texture 1-5 in Figure 2.18) and type I NRTs (texture 6-43 in Figure 2.18 and 2.19). For the results of near-regular synthesis algorithm, the lattices of regular textures are automatically extracted, and those of NRTs are specified manually. The regularity preservation test and the user evaluation test were used to evaluate the synthesis results. These two tests are complementary to each other. The former is more objective since it checks if the global regularity is preserved and quantitatively compares the geometry and appearance regularity of an input texture and its synthesized texture; the latter is subjective, but it examines the overall quality of the synthesized textures, including 
color/intensity, statistical variations, and structures, in terms of human perception. We summarized the evaluation results of these four synthesis algorithms on regular and near-regular textures in Table 2.3 and 2.4.

Regularity Preservation Test. In the regularity preservation test, we first visually determined if the global regularity of an input texture is faithfully preserved in synthesized textures. We found that all four synthesis algorithms preserve the global regularity in the tested regular textures; however, for NRTs, their performance is quite different. The success rates of the graph cut approach, the near-regular synthesis approach, the regularized patch-based approach, and the patch-based approach are 55\%, 95\%, $86 \%$, and $23 \%$, respectively. We also included six synthesis results of the image quilting approach [Efros and Freeman, 2001] ${ }^{2}$ (Figures B.15 and B.16). Among the six tested textures, only two synthesized results preserved the global regularity(Figure B.15(f) and Figure B.16(b)).

In our regularity preservation test, the underlying lattice of an NRT was manually specified. $\mathbf{t}_{1}$ and $\mathbf{t}_{\mathbf{2}}$ were computed from the input texture, and used as the values of $\mathbf{t}_{1}$ and $\mathbf{t}_{\mathbf{2}}$ in Equation (2.2) when evaluating the geometric regularity of a synthesized texture. Note that $\mathbf{t}_{\mathbf{1}}$ and $\mathbf{t}_{\mathbf{2}}$ are only used for computing the geometric regularity, none of the synthesis algorithms in this comparison study optimizes the $g$ score of a synthesized texture to match that of the input texture, i.e., the synthesis process is independent of the regularity measurement.

The $\mathrm{g}$ score and a score of all regular and near-regular textures are listed in Figures B.2-B.14. The regularity difference between the input texture and the synthesized texture of each algorithm are shown in Table 2.3 $3^{3}$. Figures 2.16 and 2.17 show the bar diagram of the $g$ score difference and the a score difference of each texture respectively. For some textures whose geometric regularity is totally broken, their g score error is denoted by the maximum g score error of all textures since there is no lattice structure in these textures (for example, the graph cut, regularized, and patch-based results of Texture 13, the patch-based result of Texture 16, and the graph cut result of Texture

\footnotetext{
${ }^{2}$ We tried to ask the authors to test their algorithm on our testing textures, but they were not able to run the experiment upon the completion of this thesis.

${ }^{3}$ It is possible to compute the error in percentages instead of differences. However, since the ga-scores of some input textures are zero, the error percentages cannot be computed in this case. We therefore adopt the ga-score differences in comparison for consistency.
} 
Table 2.3. Results of regularity preservation test on regular textures (top) and near-regular textures (bottom), where $\checkmark$ denotes that regularity is preserved, and $\times$ denotes not. $g$ err. and $a$ err. are the difference of the geometry and appearance regularity between a synthesized texture and its input texture. The $\mathrm{g}$ score error with asterisk denotes that no lattice structure exists in the texture and the maximum score error is assigned.

\begin{tabular}{|c|c|c|c|c|c|c|c|c|c|c|c|c|c|}
\hline \multicolumn{2}{|c|}{ Regular Textures } & \multicolumn{3}{|c|}{ Graph cut } & \multicolumn{3}{|c|}{$\begin{array}{c}\text { Near-regular } \\
\text { synthesis }\end{array}$} & \multicolumn{3}{|c|}{$\begin{array}{l}\text { Regularized } \\
\text { patch-based }\end{array}$} & \multicolumn{3}{|c|}{ Patch-based } \\
\hline Textures & Description & & g err. & a err. & & g err. & a err. & & g err. & a err. & & g err. & a err. \\
\hline 1 & wallpaper & $\checkmark$ & 0.00 & 0.00 & $\checkmark$ & 0.00 & 0.00 & $\checkmark$ & 0.00 & 0.00 & $\checkmark$ & 0.00 & 0.00 \\
\hline 2 & wallpaper & $\checkmark$ & 0.00 & 0.00 & $\checkmark$ & 0.00 & 0.00 & $\checkmark$ & 0.00 & 0.00 & $\checkmark$ & 0.00 & 0.00 \\
\hline 3 & wallpaper & $\checkmark$ & 0.00 & 0.00 & $\checkmark$ & 0.00 & 0.00 & $\checkmark$ & 0.00 & 0.00 & $\checkmark$ & 0.00 & 0.00 \\
\hline 4 & jigsaw puzzle & $\checkmark$ & 0.29 & 0.03 & $\checkmark$ & 0.21 & 0.02 & $\checkmark$ & 0.00 & 0.05 & $\checkmark$ & 0.24 & 0.04 \\
\hline 5 & pavement tiles & $\checkmark$ & 0.00 & 0.04 & $\checkmark$ & 0.00 & 0.04 & $\checkmark$ & 0.00 & 0.00 & $\checkmark$ & 0.00 & 0.01 \\
\hline \multicolumn{2}{|c|}{ success rate $(\%)$, average } & 100 & 0.06 & 0.014 & 100 & 0.04 & 0.013 & 100 & 0 & 0.010 & 100 & 0.05 & 0.010 \\
\hline
\end{tabular}

\begin{tabular}{|c|c|c|c|c|c|c|c|c|c|c|c|c|c|}
\hline \multicolumn{2}{|c|}{ Near-regular Textures } & \multicolumn{3}{|c|}{ Graph cut } & \multicolumn{3}{|c|}{$\begin{array}{l}\text { Near-regular } \\
\text { synthesis }\end{array}$} & \multicolumn{3}{|c|}{$\begin{array}{l}\text { Regularized } \\
\text { patch-based }\end{array}$} & \multicolumn{3}{|c|}{ Patch-based } \\
\hline Textures & Description & & g err. & a err. & & g err. & a err. & & g err. & a err. & & g err. & a err. \\
\hline 6 & punched card & $\checkmark$ & 0.4 & 0.07 & $\checkmark$ & 0.0 & 0.03 & $\checkmark$ & 0.4 & 0.07 & $x$ & 1.1 & 0.12 \\
\hline 7 & hexagonal net & $\checkmark$ & 0.0 & 0.03 & $\checkmark$ & 0.0 & 0.00 & $\checkmark$ & 0.0 & 0.10 & $\checkmark$ & 0.0 & 0.05 \\
\hline 8 & metal & $x$ & 9.0 & 0.00 & $\checkmark$ & 0.0 & 0.00 & $\checkmark$ & 0.0 & 0.00 & $x$ & 10.9 & 0.00 \\
\hline 9 & ceramic tiles & $\checkmark$ & 0.3 & 0.00 & $\checkmark$ & 0.4 & 0.00 & $\checkmark$ & 1.5 & 0.00 & $x$ & 2.4 & 0.00 \\
\hline 10 & fish tiles & $\checkmark$ & 0.1 & 0.00 & $\checkmark$ & 0.0 & 0.03 & $\checkmark$ & 0.7 & 0.02 & $x$ & 9.1 & 0.07 \\
\hline 11 & wall & $\checkmark$ & 0.0 & 0.06 & $\checkmark$ & 0.0 & 0.07 & $\checkmark$ & 0.0 & 0.08 & $x$ & 4.7 & 0.15 \\
\hline 12 & squares & $x$ & 6.4 & 0.05 & $\checkmark$ & 0.1 & 0.00 & $\checkmark$ & 0.1 & 0.02 & $x$ & 11.1 & 0.04 \\
\hline 13 & pavement tiles & $x$ & $27.1^{*}$ & 0.00 & $\checkmark$ & 0.0 & 0.00 & $x$ & $27.1^{*}$ & 0.00 & $x$ & $27.1^{*}$ & 0.00 \\
\hline 14 & cans & $\checkmark$ & 0.6 & 0.02 & $\checkmark$ & 0.1 & 0.02 & $\checkmark$ & 0.1 & 0.01 & $x$ & 0.7 & 0.02 \\
\hline 15 & swirl & $\checkmark$ & 1.0 & 0.04 & $\checkmark$ & 0.6 & 0.02 & $\checkmark$ & 1.3 & 0.03 & $x$ & 1.8 & 0.03 \\
\hline 16 & basket & $\begin{array}{lll} & \end{array}$ & 0.4 & 0.01 & $\checkmark$ & 0.0 & 0.01 & $\checkmark$ & 0.5 & 0.01 & $x$ & $27.1^{*}$ & 0.02 \\
\hline 17 & fabric & $\checkmark$ & 0.0 & 0.03 & $\checkmark$ & 0.0 & 0.04 & $\checkmark$ & 0.0 & 0.02 & $\checkmark$ & 0.0 & 0.04 \\
\hline 18 & fabric & $\checkmark$ & 0.0 & 0.03 & $\checkmark$ & 0.0 & 0.03 & $\checkmark$ & 0.0 & 0.02 & $\checkmark$ & 0.0 & 0.03 \\
\hline 19 & fabric & $\checkmark$ & 0.0 & 0.02 & $\checkmark$ & 0.0 & 0.02 & 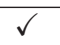 & 0.0 & 0.01 & $\checkmark$ & 0.0 & 0.01 \\
\hline 20 & knotted mat & $\checkmark$ & 3.8 & 0.02 & $\checkmark$ & 0.9 & 0.02 & $\checkmark$ & 5.4 & 0.01 & $x$ & 6.3 & 0.09 \\
\hline 21 & pie & $x$ & 2.9 & 0.00 & $\checkmark$ & 2.3 & 0.00 & $x$ & 6.7 & 0.00 & $x$ & $27.1^{*}$ & 0.00 \\
\hline 22 & fabric & $\checkmark$ & 0.1 & 0.03 & $\checkmark$ & 0.6 & 0.03 & $\checkmark$ & 0.5 & 0.02 & $x$ & 15.8 & 0.12 \\
\hline 23 & toothpastes & $\begin{array}{lll} & \end{array}$ & 0.0 & 0.05 & $\checkmark$ & 0.0 & 0.07 & $\checkmark$ & 0.0 & 0.01 & $\checkmark$ & 0.0 & 0.02 \\
\hline 24 & windows & $\checkmark$ & 0.0 & 0.08 & $\checkmark$ & 0.0 & 0.05 & $\checkmark$ & 0.0 & 0.04 & $x$ & 15.3 & 0.06 \\
\hline 25 & windows & $\begin{array}{lll}v^{\prime} & & \end{array}$ & 0.0 & 0.04 & $\checkmark$ & 0.0 & 0.04 & $\checkmark$ & 0.0 & 0.00 & $x$ & 27.1 & 0.06 \\
\hline 26 & fabric & $x$ & 0.3 & 0.00 & $\checkmark$ & 0.2 & 0.00 & $\checkmark$ & 0.7 & 0.03 & $x$ & 2.9 & 0.01 \\
\hline 27 & basket & $\checkmark$ & 0.5 & 0.03 & $\checkmark$ & 0.3 & 0.02 & $x$ & 2.5 & 0.01 & $x$ & $27.1^{*}$ & 0.07 \\
\hline 28 & fabric & $\begin{array}{lll} & \end{array}$ & 0.2 & 0.00 & $\checkmark$ & 0.2 & 0.00 & \multicolumn{3}{|c|}{$\mathrm{N} / \mathrm{A}$} & \multicolumn{3}{|c|}{$\mathrm{N} / \mathrm{A}$} \\
\hline 29 & squares\&hexagons & $x$ & 13.7 & 0.03 & $x$ & 0.0 & 0.00 & \multicolumn{3}{|c|}{ N/A } & \multicolumn{3}{|c|}{ N/A } \\
\hline 30 & mosaic & $x$ & 0.2 & 0.08 & $\checkmark$ & 0.1 & 0.00 & \multirow{2}{*}{\multicolumn{3}{|c|}{$\begin{array}{l}\text { N/A } \\
\text { N/A }\end{array}$}} & \multirow{2}{*}{\multicolumn{3}{|c|}{ N/A }} \\
\hline 31 & jigsaw puzzle & $\checkmark$ & 0.9 & 0.03 & $x$ & 1.8 & 0.02 & & & & \multirow{2}{*}{\multicolumn{3}{|c|}{$\frac{\mathrm{N} / \mathrm{A}}{\mathrm{N} / \mathrm{A}}$}} \\
\hline 32 & fabric & $x$ & 24.4 & 0.01 & $\checkmark$ & 0.2 & 0.01 & \multicolumn{3}{|c|}{$\mathrm{N} / \mathrm{A}$} & & & \\
\hline 33 & cracker & $x$ & 4.3 & 0.00 & $\checkmark$ & 0.4 & 0.01 & \multicolumn{3}{|c|}{ N/A } & \multicolumn{3}{|c|}{ N/A } \\
\hline 34 & brick wall & $x$ & 0.7 & 0.03 & $\checkmark$ & 0.2 & 0.03 & \multirow{2}{*}{\multicolumn{3}{|c|}{$\mathrm{N} / \mathrm{A}$}} & \multicolumn{3}{|c|}{$\mathrm{N} / \mathrm{A}$} \\
\hline 35 & brick wall & $x$ & 0.6 & 0.00 & $\checkmark$ & 0.0 & 0.01 & & & & \multicolumn{3}{|c|}{ N/A } \\
\hline 36 & brick wall & $x$ & 2.3 & 0.00 & $\checkmark$ & 0.3 & 0.00 & \multicolumn{3}{|c|}{ N/A } & & $\mathrm{N} / \mathrm{A}$ & \\
\hline 37 & brick wall & $\checkmark$ & 0.1 & 0.01 & $\checkmark$ & 0.1 & 0.00 & \multicolumn{3}{|c|}{$\mathrm{N} / \mathrm{A}$} & \multicolumn{3}{|c|}{$\mathrm{N} / \mathrm{A}$} \\
\hline 38 & brick wall & $x$ & $27.1^{*}$ & 0.00 & $\checkmark$ & 0.0 & 0.00 & \multicolumn{3}{|c|}{ N/A } & & $\mathrm{N} / \mathrm{A}$ & \\
\hline 39 & brick wall & $x$ & 1.2 & 0.01 & $\checkmark$ & 0.2 & 0.01 & & $\mathrm{~N} / \mathrm{A}$ & & & $\mathrm{N} / \mathrm{A}$ & \\
\hline 40 & carpet & $\checkmark$ & 0.0 & 0.00 & $\checkmark$ & 0.0 & 0.00 & & $\mathrm{~N} / \mathrm{A}$ & & & $\mathrm{N} / \mathrm{A}$ & \\
\hline 41 & rug & $x$ & 7.6 & 0.02 & $\checkmark$ & 1.1 & 0.01 & & $\mathrm{~N} / \mathrm{A}$ & & & $\mathrm{N} / \mathrm{A}$ & \\
\hline 3842 & rug & $x$ & 7.2 & 0.03 & $\checkmark$ & 0.3 & 0.02 & & $\mathrm{~N} / \mathrm{A}$ & & & $\mathrm{N} / \mathrm{A}$ & \\
\hline 43 & cans & $x$ & 0.2 & 0.01 & $\checkmark$ & 0.2 & 0.01 & & $\mathrm{~N} / \mathrm{A}$ & & & $\mathrm{N} / \mathrm{A}$ & \\
\hline succes & s rate $(\%)$, average & 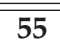 & 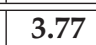 & $\overline{c 0.023}$ & 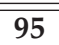 & 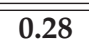 & $\bar{~} 0.017$ & 86 & 2.16 & 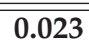 & 23 & $\begin{array}{l}9.88 \\
\end{array}$ & 0.046 \\
\hline
\end{tabular}


38). From Table 2.3, Figures 2.16 and 2.17, one can find that the NRT synthesis approach performs better than the graph cut approach, the regularized patch-based approach and the patch-based approach. In particular, the NRT synthesis approach has much smaller geometric regularity error than the other three approaches on NRTs.

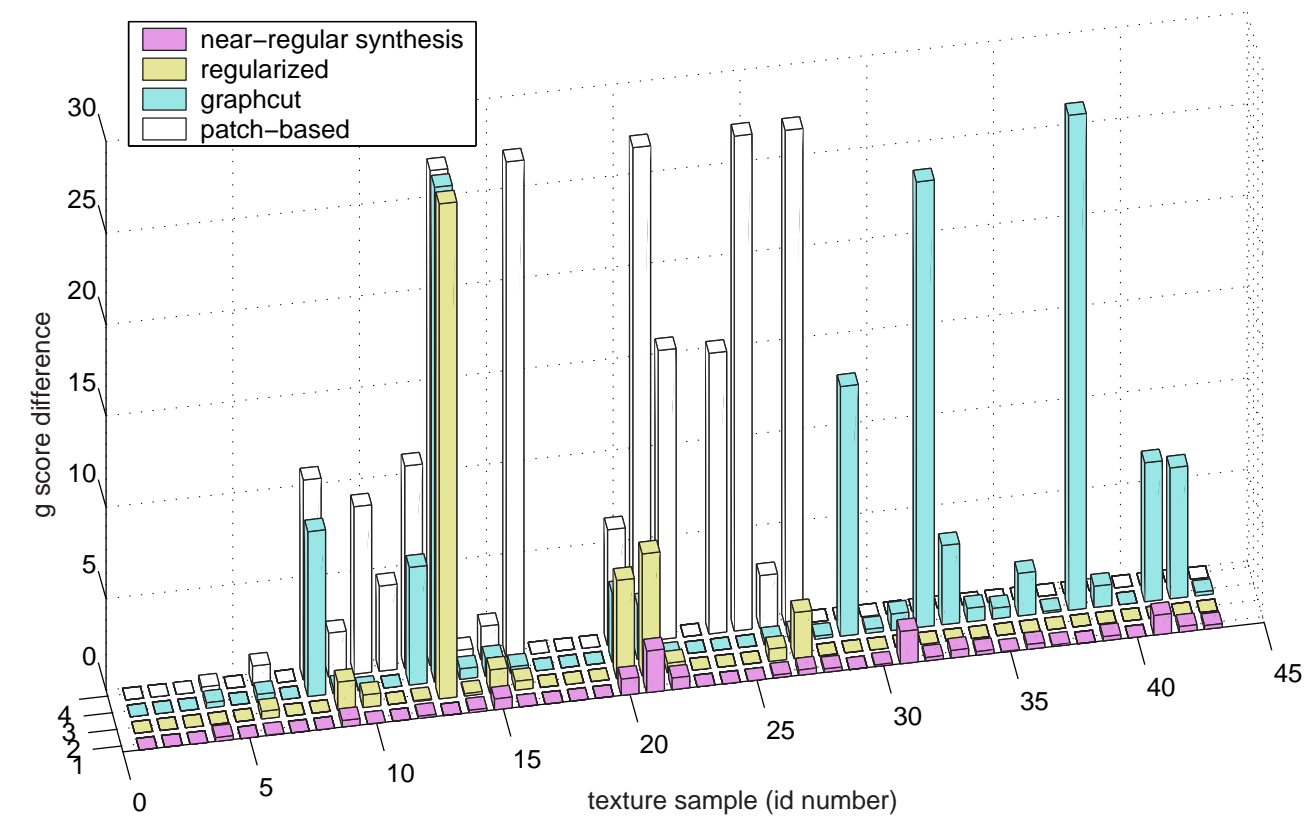

Figure 2.16. The g score errors of synthesized textures, where the $x$-axis is the figure number of the texture, and the z-axis is the $\mathrm{g}$ score difference between a synthesized texture and its original input texture. The numeric value of $g$ score difference of each texture is listed in Table 2.3.

Analysis of Algorithms Based on the Regularity Preservation Test. It is important to respect the global regularity of an NRT during synthesis. The graph cut approach [Kwatra et al., 2003], near-regular synthesis [Liu et al., 2005], the patch-based approach [Liang et al., 2001b,a] and the regularized patch-based approach handle global regularity in different ways and levels. The patch-based approach does not have any explicit mechanism to preserve the regularity (see Table 2.2). Although one can set an appropriate patch size and placement offset for regular textures, the patch-based approach cannot handle NRTs well because its patch extraction algorithm does not utilize global regularity to constrain the sampling position at the lattice points; it increases the chance that the best matched patch may not align with the boundary of a texture element. In the worst situations, this may cause the patch-based approach to totally break 


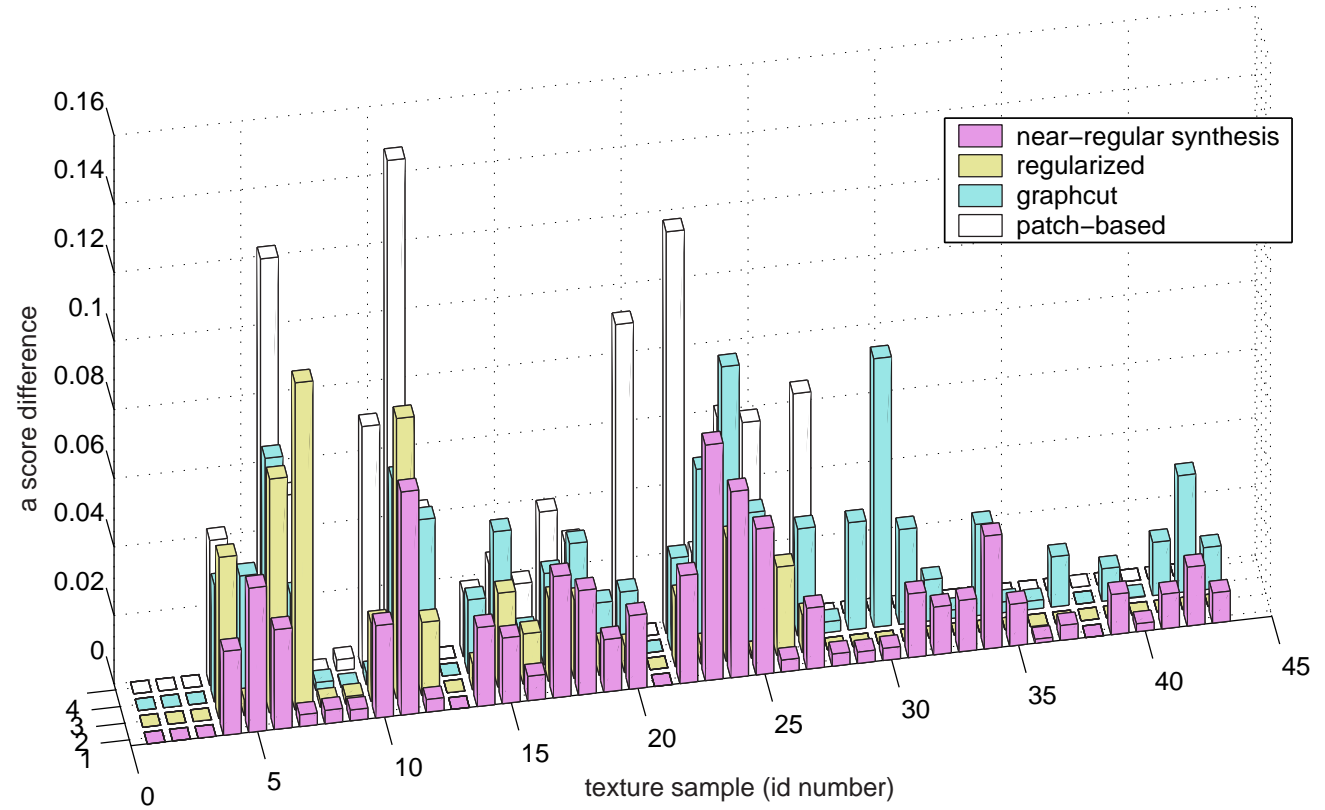

Figure 2.17. The a score errors of synthesized textures, where the $x$-axis is the figure number of the texture, and the z-axis is the a score difference between a synthesized texture and its original input texture. The numeric value of the a score difference of each texture is listed in Table 2.3.

the texture elements and violate global and local regularity in synthesized textures (see textures 8, 10, 11, 12, 13 in Appendix B).

Unlike the patch-based approach, the regularized patch-based approach utilizes a user-specified lattice in the synthesis process (Table 2.2). This lattice information is used to set the patch shape/size, patch extraction and placement locations. This helps to locate the tiles correctly in the input texture and to paste the tiles at right positions in the synthesized texture. Therefore, the regularized patch-based approach can preserve the global regularity much better than the patch-based approach.

The graph cut approach attempts to handle the global regularity by incorporating a local correlation technique to determine the best pasting location so that the underlying periodicity, if it exists, can be preserved (Table 2.2). This is the reason why the graph cut approach performs much better than the patch-based approach (Table 2.3). The correlation-based patch placement works well on regular textures and some nearregular textures; however, for NRTs in which the color/intensity of texture elements is not regular, the correlation technique cannot guarantee the preservation of global regularity. This is especially true when an input texture contains an interlocked structure, 
such as woven fabric or brick walls (see Texture 21, 26, 32, 34, 35, 36, 38, 39 in Appendix B). Figure B.1 shows several synthesis results of a brick wall texture by the graph cut approach with different patch placement settings. This example shows that the correlation technique does not work well on discovering the regularity of an NRT with interlocked structure. Synthesis result of the same texture by the near-regular synthesis approach is shown in Figure B.12.

The NRT synthesis approach utilizes the translational symmetry property to analyze the global regularity of NRTs. This analysis helps to identify the underlying lattice of the input texture and the lattice determines the parameters of patch size/shape, patch extraction and patch placement locations. A difference between the the regularized patch-based approach and NRT synthesis approach is that the former samples tiles without overlapping while the latter samples tiles with overlapping size at half or third quarters of a tile. There are two advantages of sampling tiles with overlapping. First, searching the best matched tile is more robust as there is a larger overlapping between tiles. Second, the overlapping can be used to adjust the pasting location so that the best matched tile is registered to the existing synthesized texture. Through use of overlapped tile sets, the NRT synthesis approach can preserve the global regularity better than the regularized patch-based approach. Moreover, the interlocked structure of an NRT is also better preserved because tiles are extracted and pasted/registered accurately at lattice points. This is the reason that the NRT outperforms the graph cut approach in brick wall textures and woven fabric textures.

User Evaluation Test. 10 subjects participated in the user evaluation test. We showed each subject the input and synthesized textures on a computer screen and the subject was asked the following question: how well is the image characteristics of the input texture faithfully preserved in the synthesized textures? The subject then gave scores to each synthesized texture. The range of score, from best to worst, is 4 to 1 . We computed the mean and standard deviation of the scores from 10 subjects for each synthesized texture. The results are listed in Table 2.4.

The standard deviation of the user scores of each texture in Table 2.4 shows how much agreement there is among different subjects when they score to a synthesized NRT. We use red texts and blue texts to denote the standard deviations of the synthesized textures that are greater than 1.15 and less than 0.33 respectively. The synthesized 
textures whose standard deviation of the user scores are greater than 1.15 are the nearregular synthesis result of Texture $41(s t d=1.25)$, the patch-based result of Texture 19 $(s t d=1.20)$ and Texture $15(s t d=1.17)$, and the graph cut synthesis result of Texture $34(s t d=1.17)$ and Texture $43(s t d=1.16)$. The larger standard variations of these textures might be because that there is no serious flaw, e.g., the regularity violation, in the synthesized textures. Therefore, the evaluation criterion is not obvious and may vary more widely among different subjects. Synthesized textures whose standard deviations of the user scores are small (less than 0.33 ) are mostly generated by the graph cut approach and the patch-based approach. With a closer inspection, we can find that these synthesized textures either receive high scores or low scores. This is not surprising since these synthesized results are either very good or very bad and different subjects tend to give similar scores to these textures.

Analysis on User Evaluation Results. We ran analysis of variance (ANOVA ${ }^{4}$ ) to analyze the user evaluation scores. There are two major findings: (1)NRT synthesis algorithm performs statistically significantly better than the other three texture synthesis algorithms (all three pair-wise comparisons have $p<0.001$ in Table 2.5); (2)the user scores are highly correlated to the degrees of regularity, i.e. the scores for regularity-preserved textures and regularity-violated textures differ statistically significantly $(\mathrm{F}(1,1398)=879.81, p<0.001$ in Table 2.6), with averages 3.4 and 1.9 respectively.

Table 2.5 summarizes the performance of four texture synthesis algorithms in the user evaluation test and Figure 2.20 shows pairwise comparisons of the near-regular synthesis approach with the other three approaches on the user evaluation scores. From Table 2.5 and Figure 2.20, it appears that the performance of the near-regular synthesis approach is statistically significantly better than the other three approaches in the user

\footnotetext{
${ }^{4}$ ANOVA is a common tool in statistical analysis. It can be used to test if the variations among data is caused by some potential factors or just by chance. The result of an ANOVA is represented by the F-ratio and p-value. The F-ratio is defined as a ratio of between-group variation to within-group variation. For example, if we want to know if the user scores of synthesized textures are affected by the respective synthesis approach, we can group the user scores of synthesized textures according to the synthesis approach. We can then compute the F-ratio to determine if the variations of user scores are totally random or there is an effect causing by synthesis approaches. The F-ratio can be thought of as a measure of how different the means of different groups are relative to the variability within each sample. The larger this value, the greater the likelihood that the differences between the means are due to something other than chance, namely real effects. The p-value reports the significance level of the effect. The lower the $\mathrm{p}$-value, the more significant the effect. An effect is usually considered to be statistically significant if $p<0.05$ or $p<0.01$. For more details about ANOVA, please see [Neter et al., 1996].
} 
Table 2.4. Results of user evaluation on regular textures (top) and near-regular textures (bottom), where $\checkmark$ denotes that regularity is preserved, and $\times$ denotes not. The numbers in the table are the mean and standard deviation of scores given by 10 subjects. The range of score, from best to worst, is 4 to 1 . The standard deviations of the synthesized textures that are greater than 1.15 and less than 0.33 are shown in red texts and blue texts respectively.

\begin{tabular}{|c|c|c|c|c|c|c|c|c|c|c|c|c|c|}
\hline \multicolumn{9}{|c|}{ Regular Textures } & \multicolumn{3}{c|}{$\begin{array}{c}\text { Near-regular } \\
\text { synthesis }\end{array}$} & \multicolumn{3}{c|}{$\begin{array}{c}\text { Regularized } \\
\text { patch-based }\end{array}$} & \multicolumn{3}{c|}{ Patch-based } \\
\hline Textures & Description & & mean & std & & mean & std & & mean & std & & mean & std \\
\hline 1 & wallpaper & $\checkmark$ & 3.5 & 0.71 & $\checkmark$ & 3.6 & 0.70 & $\checkmark$ & 3.3 & 0.95 & $\checkmark$ & 3.4 & 0.84 \\
\hline 2 & wallpaper & $\checkmark$ & 3.6 & 0.70 & $\checkmark$ & 3.6 & 0.84 & $\checkmark$ & 3.5 & 0.71 & $\checkmark$ & 3.5 & 0.71 \\
\hline 3 & wallpaper & $\checkmark$ & 3.8 & 0.42 & $\checkmark$ & 3.8 & 0.42 & $\checkmark$ & 3.8 & 0.42 & $\checkmark$ & 3.8 & 0.42 \\
\hline 4 & jigsaw puzzle & $\checkmark$ & 3.9 & 0.32 & $\checkmark$ & 3.8 & 0.42 & $\checkmark$ & 3.6 & 0.70 & $\checkmark$ & 3.6 & 0.70 \\
\hline 5 & pavement tiles & $\checkmark$ & 3.9 & 0.32 & $\checkmark$ & 3.6 & 0.52 & $\checkmark$ & 3.7 & 0.48 & $\checkmark$ & 3.7 & 0.48 \\
\hline \hline
\end{tabular}

\begin{tabular}{|c|c|c|c|c|c|c|c|c|c|c|c|c|c|}
\hline \multicolumn{2}{|c|}{ Near-Regular Textures } & \multicolumn{3}{|c|}{ Graph cut } & \multicolumn{3}{|c|}{$\begin{array}{c}\text { Near-regular } \\
\text { synthesis }\end{array}$} & \multicolumn{3}{|c|}{$\begin{array}{l}\text { Regularized } \\
\text { patch-based }\end{array}$} & \multicolumn{3}{|c|}{ Patch-based } \\
\hline Textures & Description & & mean & std & & mean & std & & mean & std & & mean & std \\
\hline 6 & punched card & $\checkmark$ & 3.8 & 0.63 & $\checkmark$ & 3.1 & 0.88 & $\checkmark$ & 2.6 & 1.07 & $x$ & 1.5 & 0.85 \\
\hline 7 & hexagonal net & $\checkmark$ & 3.7 & 0.48 & $\checkmark$ & 3.6 & 0.70 & $\checkmark$ & 3.4 & 0.84 & $\checkmark$ & 3.5 & 0.71 \\
\hline 8 & metal & $x$ & 1.5 & 0.97 & $\checkmark$ & 3.3 & 0.82 & $\checkmark$ & 3.4 & 0.70 & $x$ & 1.3 & 0.67 \\
\hline 9 & ceramic tiles & $\checkmark$ & 3.5 & 0.71 & $\checkmark$ & 3.1 & 0.88 & $\checkmark$ & 2.8 & 0.79 & $x$ & 2.4 & 0.97 \\
\hline 10 & fish tiles & $\checkmark$ & 3.7 & 0.48 & $\checkmark$ & 3.7 & 0.48 & $\checkmark$ & 2.6 & 0.84 & $x$ & 1.1 & 0.32 \\
\hline 11 & wall & $\checkmark$ & 3.9 & 0.32 & $\checkmark$ & 3.3 & 0.82 & $\checkmark$ & 3.7 & 0.48 & $x$ & 1.0 & 0.00 \\
\hline 12 & squares & $x$ & 1.1 & 0.32 & $\checkmark$ & 3.4 & 0.52 & $\checkmark$ & 3.5 & 0.71 & $x$ & 1.1 & 0.32 \\
\hline 13 & pavement tiles & $x$ & 1.4 & 0.52 & $\checkmark$ & 3.6 & 0.52 & $x$ & 3.2 & 0.63 & $x$ & 1.1 & 0.32 \\
\hline 14 & cans & $\checkmark$ & 3.0 & 0.82 & $\checkmark$ & 3.0 & 1.05 & $\checkmark$ & 3.5 & 0.53 & $x$ & 2.5 & 0.97 \\
\hline 15 & swirl & $\checkmark$ & 3.4 & 0.84 & $\checkmark$ & 3.4 & 0.84 & $\checkmark$ & 2.6 & 1.07 & $x$ & 2.6 & 1.17 \\
\hline 16 & basket & $\checkmark$ & 2.4 & 0.97 & $\checkmark$ & 3.3 & 0.82 & $\checkmark$ & 2.9 & 0.57 & $x$ & 1.6 & 1.07 \\
\hline 17 & fabric & $\checkmark$ & 3.9 & 0.32 & $\checkmark$ & 3.7 & 0.48 & $\checkmark$ & 3.5 & 0.71 & $\checkmark$ & 3.6 & 0.70 \\
\hline 18 & fabric & $\checkmark$ & 3.7 & 0.48 & $\checkmark$ & 3.4 & 0.84 & $\checkmark$ & 2.8 & 0.92 & $\checkmark$ & 2.9 & 0.88 \\
\hline 19 & fabric & $\checkmark$ & 3.8 & 0.42 & $\checkmark$ & 3.4 & 0.97 & $\checkmark$ & 3.0 & 0.94 & $\checkmark$ & 2.9 & 1.20 \\
\hline 20 & knotted mat & $\checkmark$ & 3.8 & 0.42 & $\checkmark$ & 3.5 & 0.71 & $\checkmark$ & 3.4 & 0.84 & $x$ & 1.3 & 0.48 \\
\hline 21 & pie & $x$ & 2.9 & 0.99 & $\checkmark$ & 3.6 & 0.70 & $x$ & 2.3 & 0.48 & $x$ & 1.1 & 0.32 \\
\hline 22 & fabric & $\checkmark$ & 3.0 & 0.94 & $\checkmark$ & 3.7 & 0.48 & $\checkmark$ & 3.2 & 0.63 & $x$ & 1.4 & 0.97 \\
\hline 23 & toothpastes & $\checkmark$ & 3.2 & 0.92 & $\checkmark$ & 3.8 & 0.42 & $\checkmark$ & 3.5 & 0.71 & $\checkmark$ & 2.8 & 1.03 \\
\hline 24 & windows & $\checkmark$ & 3.4 & 0.97 & $\checkmark$ & 2.6 & 0.97 & $\checkmark$ & 2.4 & 1.07 & $x$ & 1.3 & 0.48 \\
\hline 25 & windows & $\checkmark$ & 3.3 & 0.82 & $\checkmark$ & 3.7 & 0.48 & $\checkmark$ & 3.5 & 0.71 & $x$ & 1.2 & 0.63 \\
\hline 26 & fabric & $x$ & 3.4 & 0.70 & $\checkmark$ & 3.2 & 0.79 & $\checkmark$ & 3.0 & 0.94 & $x$ & 1.4 & 0.52 \\
\hline 27 & basket & $\checkmark$ & 3.2 & 0.63 & $\checkmark$ & 3.5 & 0.71 & $x$ & 2.2 & 0.63 & $x$ & 1.1 & 0.32 \\
\hline 28 & fabric & $\checkmark$ & 3.9 & 0.32 & $\checkmark$ & 3.6 & 0.52 & \multicolumn{3}{|c|}{$\mathrm{N} / \mathrm{A}$} & \multicolumn{3}{|c|}{$\mathrm{N} / \mathrm{A}$} \\
\hline 29 & squares\&hexagons & $x$ & 1.5 & 0.97 & $x$ & 3.1 & 0.74 & \multicolumn{3}{|c|}{$\mathrm{N} / \mathrm{A}$} & \multicolumn{3}{|c|}{$\mathrm{N} / \mathrm{A}$} \\
\hline 30 & mosaic & $x$ & 2.2 & 1.03 & $\checkmark$ & 3.6 & 0.70 & \multicolumn{3}{|c|}{$\mathrm{N} / \mathrm{A}$} & \multicolumn{3}{|c|}{$\mathrm{N} / \mathrm{A}$} \\
\hline 31 & jigsaw puzzle & $\checkmark$ & 3.5 & 0.53 & $x$ & 2.4 & 0.97 & \multicolumn{3}{|c|}{$\mathrm{N} / \mathrm{A}$} & \multicolumn{3}{|c|}{$\mathrm{N} / \mathrm{A}$} \\
\hline 32 & fabric & $x$ & 1.3 & 0.48 & $\checkmark$ & 3.8 & 0.42 & \multicolumn{3}{|c|}{$\mathrm{N} / \mathrm{A}$} & \multicolumn{3}{|c|}{$\mathrm{N} / \mathrm{A}$} \\
\hline 33 & cracker & $x$ & 2.2 & 0.92 & $\checkmark$ & 3.7 & 0.48 & \multicolumn{3}{|c|}{$\mathrm{N} / \mathrm{A}$} & \multicolumn{3}{|c|}{$\mathrm{N} / \mathrm{A}$} \\
\hline 34 & brick wall & $x$ & 2.6 & 1.17 & $\checkmark$ & 3.8 & 0.42 & \multicolumn{3}{|c|}{$\mathrm{N} / \mathrm{A}$} & \multicolumn{3}{|c|}{$\mathrm{N} / \mathrm{A}$} \\
\hline 35 & brick wall & $x$ & 3.4 & 0.70 & $\checkmark$ & 3.4 & 0.70 & \multicolumn{3}{|c|}{$\mathrm{N} / \mathrm{A}$} & \multicolumn{3}{|c|}{$\mathrm{N} / \mathrm{A}$} \\
\hline 36 & brick wall & $x$ & 2.9 & 0.57 & $\checkmark$ & 3.3 & 0.48 & \multicolumn{3}{|c|}{$\mathrm{N} / \mathrm{A}$} & & $\mathrm{N} / \mathrm{A}$ & \\
\hline 37 & brick wall & $\checkmark$ & 3.2 & 0.79 & $\checkmark$ & 3.6 & 0.70 & & $\mathrm{~N} / \mathrm{A}$ & & & $\mathrm{N} / \mathrm{A}$ & \\
\hline 38 & brick wall & $x$ & 1.1 & 0.32 & $\checkmark$ & 3.7 & 0.48 & & $\mathrm{~N} / \mathrm{A}$ & & & $\mathrm{N} / \mathrm{A}$ & \\
\hline 39 & brick wall & $x$ & 1.2 & 0.42 & $\checkmark$ & 3.1 & 0.88 & & $\mathrm{~N} / \mathrm{A}$ & & & $\mathrm{N} / \mathrm{A}$ & \\
\hline 40 & carpet & $\checkmark$ & 3.8 & 0.42 & $\checkmark$ & 3.7 & 0.67 & & $\mathrm{~N} / \mathrm{A}$ & & & $\mathrm{N} / \mathrm{A}$ & \\
\hline 41 & rug & $x$ & 2.3 & 0.67 & $\checkmark$ & 2.7 & 1.25 & & $\mathrm{~N} / \mathrm{A}$ & & & $\mathrm{N} / \mathrm{A}$ & \\
\hline 42 & rug & $x$ & 2.4 & 0.84 & $\checkmark$ & 3.3 & 0.48 & & $\mathrm{~N} / \mathrm{A}$ & & & $\mathrm{N} / \mathrm{A}$ & 43 \\
\hline 43 & cans & $x$ & 2.7 & 1.16 & $\checkmark$ & 3.4 & 0.70 & & $\mathrm{~N} / \mathrm{A}$ & & & $\mathrm{N} / \mathrm{A}$ & \\
\hline success & rate $(\%)$, mean, std & $\overline{55}$ & 2.9 & $\overline{\overline{1.13}}$ & 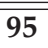 & $\begin{array}{l}3.4 \\
\end{array}$ & $\overline{0.76}$ & 86 & $\begin{array}{l}3.0 \\
\end{array}$ & 0.87 & 23 & $\begin{array}{ll}1.9 \\
\end{array}$ & $\overline{1.10}$ \\
\hline
\end{tabular}


Table 2.5. Summary of the user evaluation test on four texture synthesis algorithms, including regular and near-regular textures. The mean and standard variation of each algorithm is computed across different users and textures. ANOVA method [Neter et al., 1996] (section 2.5.2) is used to verify if there is statistically significant difference between the mean of the near-regular synthesis algorithm and the means of the other three. We did pairwise comparisons and the differences of means in all three comparisons are statistically significant.

\begin{tabular}{|c|c|c|c|c|}
\hline Algorithms & Graph cut & $\begin{array}{c}\text { Near-regular } \\
\text { synthesis }\end{array}$ & $\begin{array}{c}\text { Regularized } \\
\text { patch-based }\end{array}$ & Patch-based \\
\hline mean score & 3.0 & 3.4 & 3.1 & 2.2 \\
\hline standard deviation & 1.11 & 0.75 & 0.86 & 1.24 \\
\hline standard error of the mean & 0.054 & 0.036 & 0.052 & 0.075 \\
\hline ANOVA & $\begin{array}{c}\mathrm{F}(1,858)=54.18 \\
p<0.001\end{array}$ & $\mathrm{~N} / \mathrm{A}$ & $\begin{array}{c}\mathrm{F}(1,698)=27.37 \\
p<0.001\end{array}$ & $\begin{array}{c}\mathrm{F}(1,698)=315.95 \\
p<0.001\end{array}$ \\
\hline
\end{tabular}

evaluation test. The NRT synthesis approach not only gets higher scores but also has smaller standard deviation (including variations across different users and tested textures), which implies that the evaluation scores of the near-regular synthesis approach, from different users on different tested textures, are more consistent than the other three approaches.

Furthermore, it appears that the performance of the graph cut approach and the patch-based approach are less consistent on different NRTs than that of the near-regular synthesis approach and the regularized patch-based approach. We can observe this phenomenon from Figure 2.20, where the user scores of the graph cut approach and the patch-based approach have larger variations across different texture samples than those of the other two approaches.

The second finding in ANONA shows that global regularity is actually an important factor when humans evaluate the quality of the synthesized regular and nearregular textures. We plot the histogram of the user scores of all textures in Figure 2.21. Among the 1400 synthetic textures tested in the user evaluation test ( 10 subjects $\times$ 140 synthesized textures ), 1010 textures are regularity-preserved. The histogram of the user scores of regularity-preserved textures is plotted in green bars, while that of the regularity-violated textures is plotted in blue bars. One can observe that most of the regularity-preserved synthesized textures are located in the higher score region while most of the regularity-violated synthesized textures are located in lower score region. 
Table 2.6. Comparison of user scores of regularity-preserved and regularityviolated textures. ANOVA method [Neter et al., 1996] (section 2.5.2) is used to verify if there is a statistically significant difference between the mean of the regularity-preserved synthesized textures and that of the regularity-violated synthesized textures. This table shows that synthesized NRTs that preserves global regularity get higher user scores and the difference is statistically significant.

\begin{tabular}{|c|c|c|c|}
\hline & Regularity preserved & Regularity violated & Total \\
\hline texture counts & 1010 & 390 & 1400 \\
\hline mean score & 3.41 & 1.91 & 3.00 \\
\hline standard deviation & 0.77 & 1.03 & 1.09 \\
\hline standard error of the mean & 0.024 & 0.052 & 0.029 \\
\hline ANOVA & \multicolumn{2}{|c|}{$\mathrm{F}(1,1398)=879.81, p<0.001$} \\
\hline
\end{tabular}

From Table 2.6 and Figure 2.21, we found that the user evaluation scores have strong correlations with the preservation of global regularity.

Global regularity, however, is not the only criterion the humans use to evaluate the quality of synthetic textures. Colors/intensity, statistical variations, and other factors all affect one's judgement. For example, the graph cut result of Texture 16 and the nearregular synthesis result and the regularized patch-based result of Texture 24 did not get a high score even though their regularity is preserved. Furthermore, the importance of global regularity to visual perception also varies among different people and textures. This causes discrepancy in the user evaluation test and the regularity preservation test. For instance, the violation of regularity may not be noticeable to some users (e.g., the graph cut results of Texture 26 and 35, and the near-regular synthesis result of Texture $29)$, they may still give high scores to these textures where the regularity in the input texture is not preserved in the synthesized texture.

Limitations of the NRT Synthesis Algorithm. Although the NRT synthesis algorithm performs much better than the other three texture synthesis algorithms in regularity preservation test, its major limitation is that it only works on NRTs since it is specially designed for NRT synthesis-the same limitation applicable to the regularized patch-based approach. Furthermore, the lattice extraction process is not fully automatic. Reducing the user interventions in the lattice extraction process would facilitate the application of the NRT synthesis algorithm. A semi-automatic lattice extraction algorithm that can greatly reduce user interventions will be presented in section 3.5.1. 
Another limitation of the NRT synthesis algorithm is that an input texture sample must contain at least two complete tiles so that the underlying lattice and the tile set are well defined. If no complete tile exists in the texture, the algorithm cannot produce good results. This happens in the synthesis result of Texture 29, where the input texture contains two overlapping periodic patterns (squares and hexagons), but the input texture is too small to have a complete tile that can cover a full period of the composed periodic pattern ${ }^{5}$. The NRT synthesis algorithm preserves the square pattern because it demonstrates stronger periodicity, but the hexagon net is discontinuous between squares. The thin line jigsaw puzzle texture 31 is another example of the NRT synthesis algorithm failure. In this case, the input texture does not contain a complete tile either. This can be observed from the upper-right image in which the pattern in the circled region only appears once in the texture.

When the number of tiles is small and the color/intensity of tiles are different greatly, the NRT synthesis algorithm [Liu and Tsin, 2002; Liu et al., 2005] may produce a half-brick artifact as shown in the synthesis result of Texture 39. In this case, there are only 4 tiles in the input texture, and the color/intensity of these tiles are not similar. This increases the difficulty to find a tile that can match well with the existing synthesized texture, and may generate a new brick whose color/intensity is not similar to any of that in the input texture, but a combination of two half bricks. Although stitching techniques may alleviate this problem, these techniques usually cannot find a good seam for stitching when the color/intensity of two tiles are quite different. The half-brick problem, however, can be solved by increasing the number of tiles. One way to do this is to apply Principal Component Analysis (PCA) to analyze the tile set and synthesize infinitely large set of new tiles using the PCA bases [Liu et al., 2004]. The variations of color/intensity of the synthesized tiles can be controlled through the PCA, which effectively increases the probability that there exists a tile matched with the texture being synthesized.

\subsubsection{Summary of the Comparison Study}

Our comparison study shows that NRT synthesis remains a challenge for several state-of-the-art algorithms, such as the graph cut [Kwatra et al., 2003], patch-based [Liang

\footnotetext{
${ }^{5}$ In fact, it is not guaranteed that a two-dimensional pattern composed of two periodic twodimensional patterns will remain a periodic pattern.
} 
et al., 2001a], and image quilting approaches [Efros and Freeman, 2001]. The results from the regularized patch-based approach and the NRT synthesis approach show that the global regularity of the NRTs can be better preserved if the synthesis algorithm analyzes the underlying lattice structure and uses this information in the synthesis process. In fact, the synthesis results by the NRT synthesis algorithm demonstrate that both the global regularity and local randomness of an NRT can be faithfully preserved. This reflects the results of the regularity preservation test and the user evaluation test, where the NRT synthesis approach has higher success rate in regularity preservation (95\%), smaller $g$ score error, and statistically significantly higher user scores than the graph cut approach $(p<0.001)$, the regularized patch-based approach $(p<0.001)$, and the patch-based approach $(p<0.001)$.

The user evaluation results also indicate that global regularity is actually an important factor when humans evaluate the faithfulness of the synthesized regular or nearregular textures. The mean user score of regularity-preserved textures and that of the regularity-violated are statistically significantly different $(p<0.001)$.

The comparison study further confirms that it is essential to develop specialized algorithms for NRTs, which respect the nature of NRTs, to handle NRTs. It is indeed one of the motivations of this thesis work. 


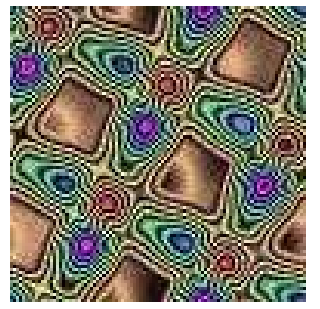

$1(\mathrm{~g}, \mathrm{a})=(0.00,0.00)$

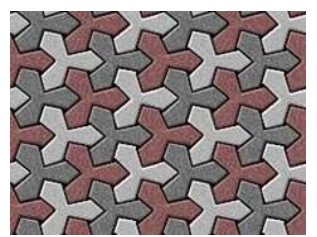

$5(\mathrm{~g}, \mathrm{a})=(0.00,0.10)$

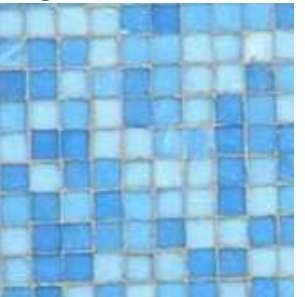

$9(\mathrm{~g}, \mathrm{a})=(0.17,0.09)$

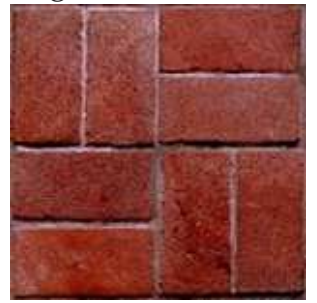

$13(\mathrm{~g}, \mathrm{a})=(0.00,0.00)$ Ko:

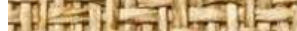
Tim: Tper $7 \rightarrow$ a $\rightarrow$ in

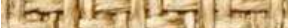

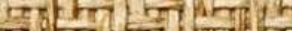

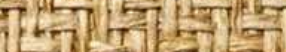

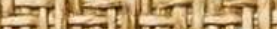

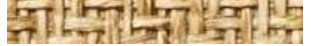

$17(\mathrm{~g}, \mathrm{a})=(0.00,0.13)$

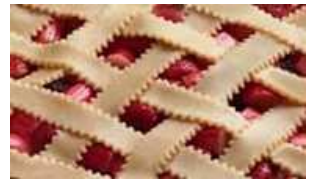

$21(\mathrm{~g}, \mathrm{a})=(0.39,0.00)$

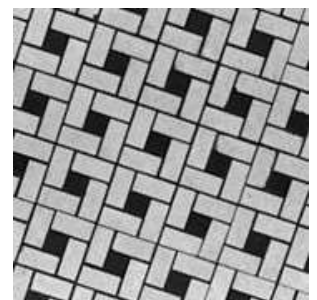

$2(\mathrm{~g}, \mathrm{a})=(0.00,0.00)$

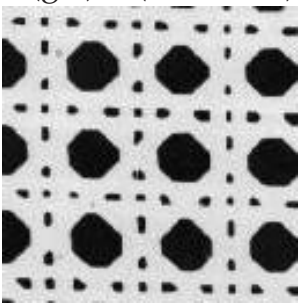

$6(\mathrm{~g}, \mathrm{a})=(0.46,0.11)$

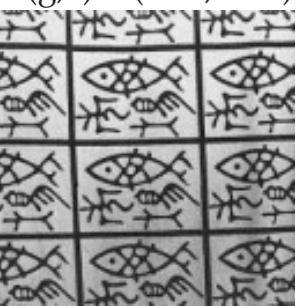

$10(\mathrm{~g}, \mathrm{a})=(0.07,0.11)$

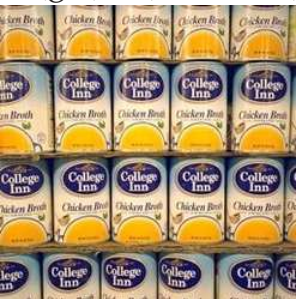

$14(\mathrm{~g}, \mathrm{a})=(0.00,0.14)$

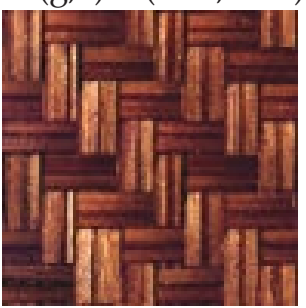

$18(g, a)=(0.00,0.09)$

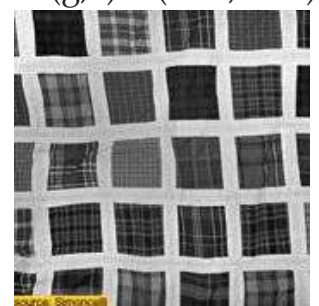

$22(\mathrm{~g}, \mathrm{a})=(0.50,0.09)$

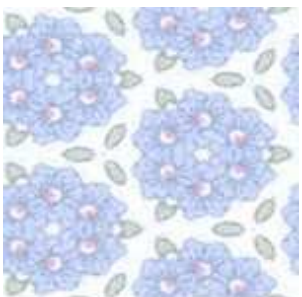

$3(\mathrm{~g}, \mathrm{a})=(0.00,0.00)$
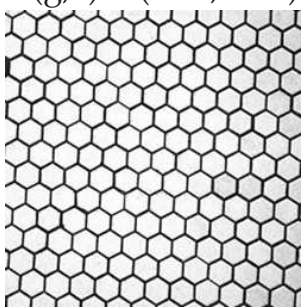

$7(\mathrm{~g}, \mathrm{a})=(0.00,0.16)$

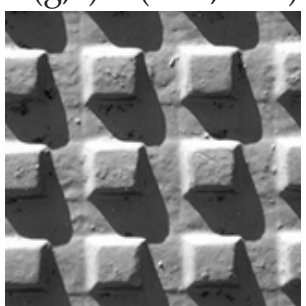

$11(\mathrm{~g}, \mathrm{a})=(0.00,0.04)$
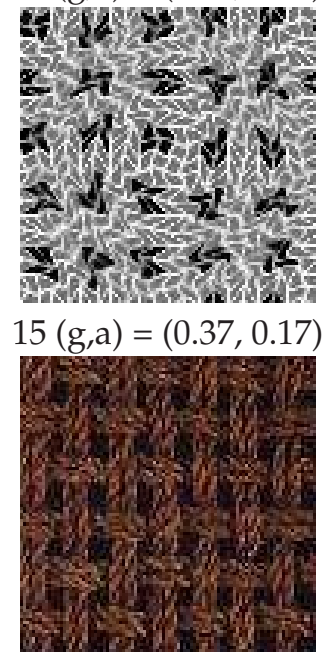

$19(\mathrm{~g}, \mathrm{a})=(0.00,0.08)$

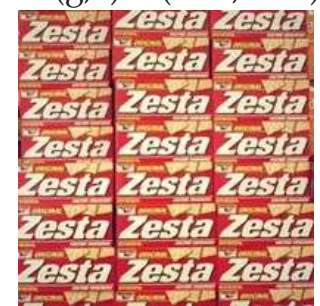

$23(\mathrm{~g}, \mathrm{a})=(0.00,0.17)$

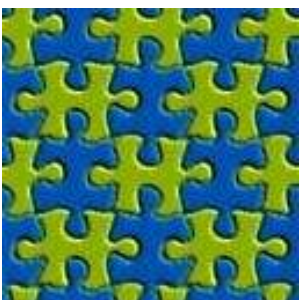

$4(\mathrm{~g}, \mathrm{a})=(0.00,0.06)$

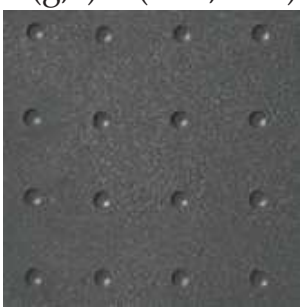

$8(\mathrm{~g}, \mathrm{a})=(0.00,0.03)$

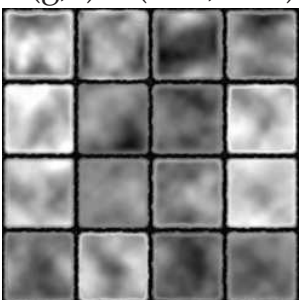

$12(\mathrm{~g}, \mathrm{a})=(0.24,0.15)$

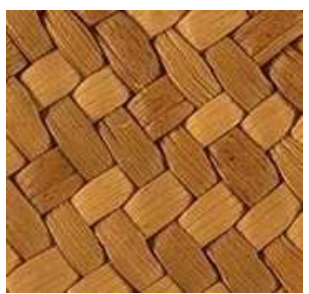

$16(\mathrm{~g}, \mathrm{a})=(0.25,0.07)$

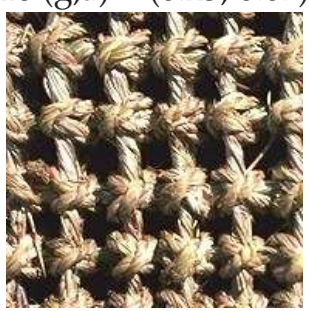

$20(\mathrm{~g}, \mathrm{a})=(0.40,0.17)$

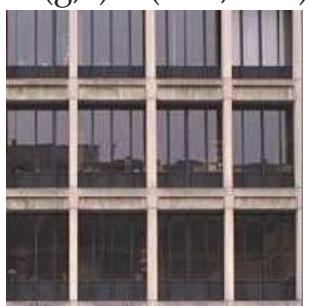

$24(\mathrm{~g}, \mathrm{a})=(0.00,0.05)$

Figure 2.18. Tested textures in our comparison study. Textures 1 to 5 are regular textures and the others are type I NRTs. The numbers in the parentheses are the ga-scores of a texture. The synthesized textures are shown in Figure B.2 - B.14 (Appendix B) 


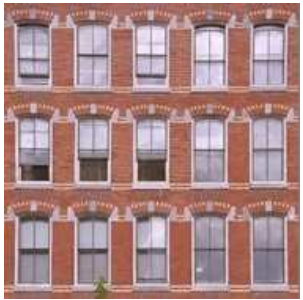

$25(\mathrm{~g}, \mathrm{a})=(0.00,0.08)$

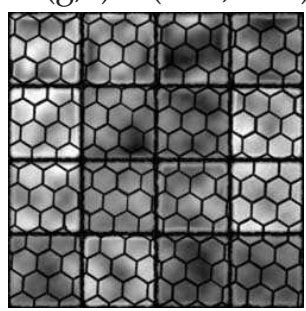

$29(\mathrm{~g}, \mathrm{a})=(0.00,0.17)$

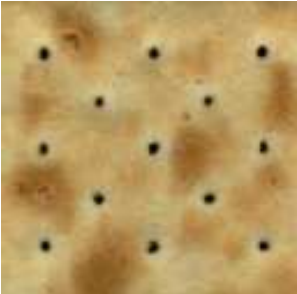

$33(\mathrm{~g}, \mathrm{a})=(0.14,0.06)$

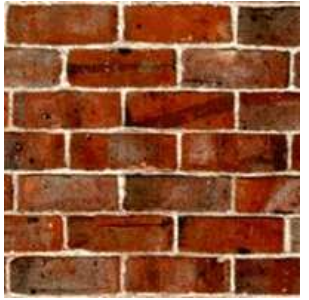

$37(\mathrm{~g}, \mathrm{a})=(0.20,0.12)$

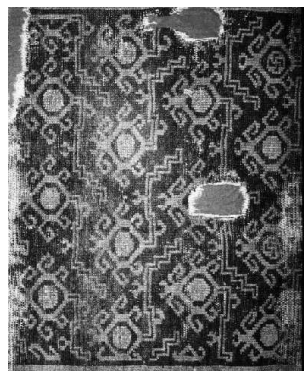

$41(\mathrm{~g}, \mathrm{a})=(2.03,0.17)$

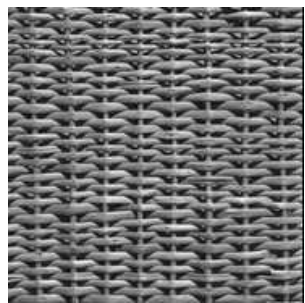

$26(\mathrm{~g}, \mathrm{a})=(0.33,0.16)$

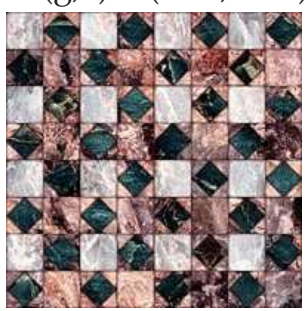

$30(\mathrm{~g}, \mathrm{a})=(0.18,0.14)$

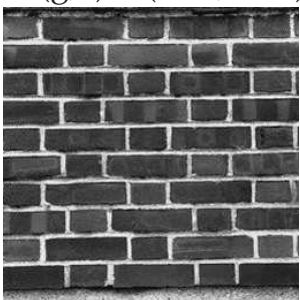

$34(\mathrm{~g}, \mathrm{a})=(0.20,0.12)$

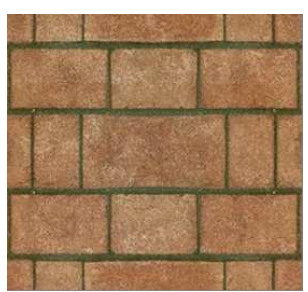

$38(\mathrm{~g}, \mathrm{a})=(0.00,0.00)$

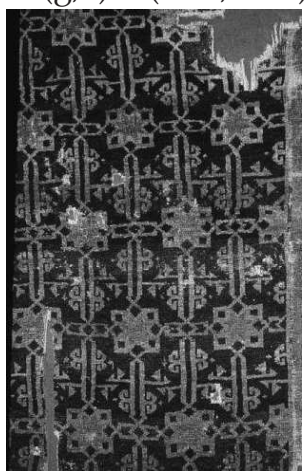

$42(\mathrm{~g}, \mathrm{a})=(0.87,0.11)$
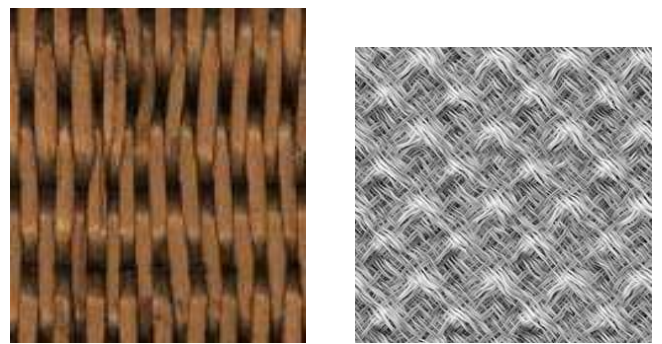

$27(\mathrm{~g}, \mathrm{a})=(0.07,0.06)$

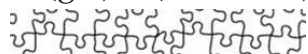

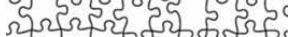

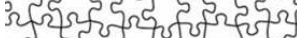

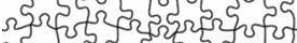

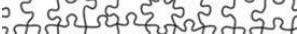

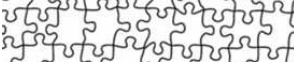

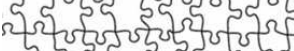

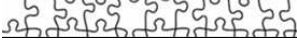

$31(\mathrm{~g}, \mathrm{a})=(1.76,0.14)$

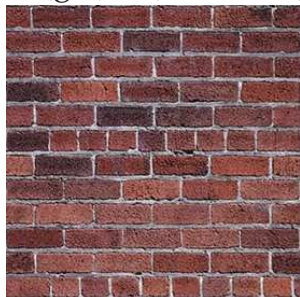

$28(\mathrm{~g}, \mathrm{a})=(0.84,0.12)$

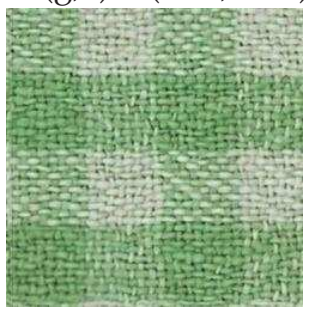

$32(\mathrm{~g}, \mathrm{a})=(0.32,0.08)$

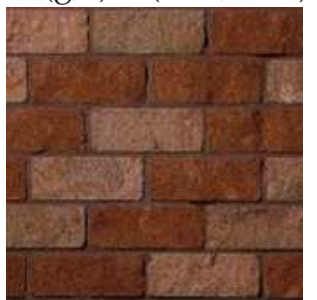

$35(\mathrm{~g}, \mathrm{a})=(0.00,0.10)$

$36(\mathrm{~g}, \mathrm{a})=(0.58,0.07)$

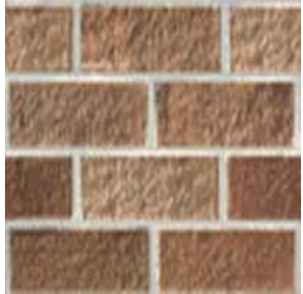

$39(\mathrm{~g}, \mathrm{a})=(0.01,0.09)$

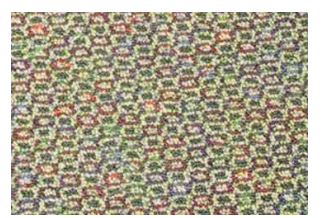

$40(\mathrm{~g}, \mathrm{a})=(0.00,0.18)$

Figure 2.19. Tested textures in our comparison study (continue). The synthesized textures are shown in Figure B.2 - B.14(Appendix B) 


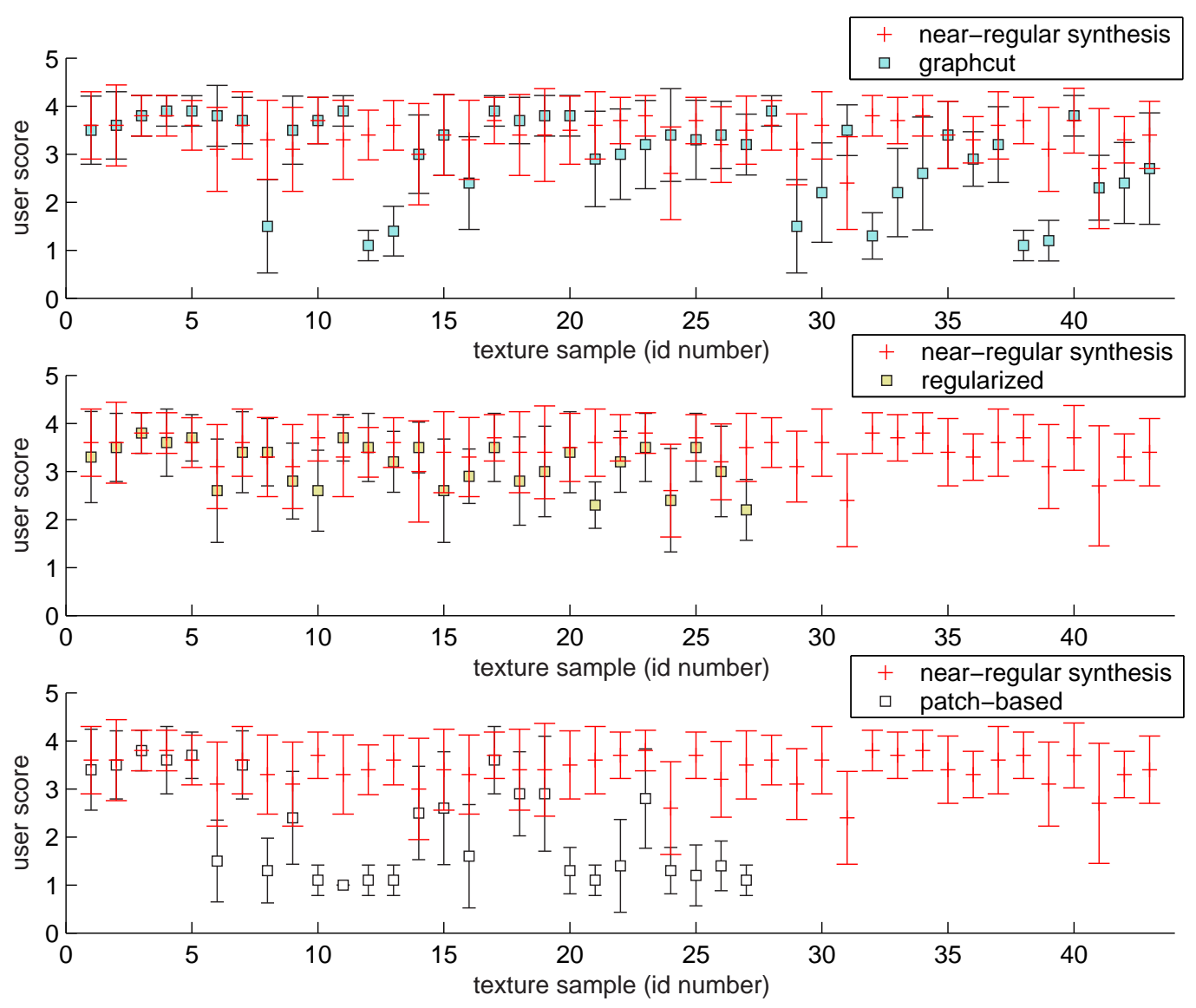

Figure 2.20. Pairwise comparison of the near-regular synthesis approach with the graph cut approach (top), the regularized patch-based approach (middle), and the patch-based approach (bottom). In each plot, the center of each vertical line represents the mean of user scores of a texture given by 10 subjects and the lower and upper bound of the line is one standard deviation on each side of the mean. From the graph cut results (top plot) and the patch-based results (bottom plot), one can observe that the variations of user scores across different textures are larger than those across different users. It appears that the performance of the graph cut approach and the patch-based approach are less consistent on different NRTs than the performance of the near-regular synthesis approach and the regularized patch-based approach. 


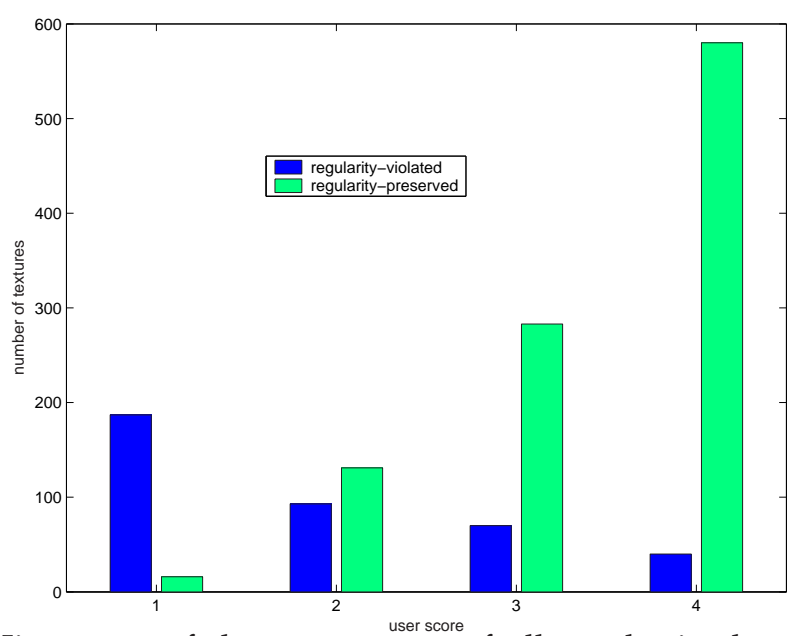

Figure 2.21. Histogram of the user scores of all synthesized textures, where green bars represent those textures whose global regularity is preserved and blue bars represent those violated. The range of user score, from best to worst, is 4 to 1 . This plot shows that preservation of global regularity is an important factor in user evaluation since most of the green bars are located in the higher score region while most of the blue bars are located in the lower score region. However, this plot also shows that the regularity preservation is not the only factor that a user evaluates a synthesized texture since there are still few regularity-preserved synthesized textures receiving low scores and some regularity-violated synthesized texture receiving high scores. This discrepancy may be because that the regularity violation is not noticeable to some users or some users count on other factors more than regularity preservation. 



\section{CHAPTER 3}

\section{Dynamic NRT Modeling, Tracking and Manipulation}

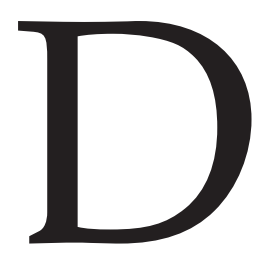

YNAMIC near-regular textures are NRTs under motion. Dynamic NRTs can be viewed as an extension to the conventional dynamic textures, which refer to a sequence of image textures that exhibit certain statistical stationary properties in time ${ }^{1}$ [Szummer and Picard, 1996; Doretto et al., 2003], such as smoke, fire, or moving water. Different from the conventional dynamic textures, dynamic NRTs possess spatial topological regularity, but their motion along the time axis may not exhibit any statistical stationarity. The spatial regularity, including geometry, topology, and appearance, brings new challenges and useful cues for handling dynamic NRTs. Figures 3.1 and 3.2 demonstate several snapshots of dynamic NRTs. While most existing work on dynamic textures addresses analysis, synthesis, or classification problems [Szummer and Picard, 1996; Bar-Joseph et al., 2001; Wei and Levoy, 2000; Kwatra et al., 2003; Doretto et al., 2003; Bhat et al., 2004; Saisan et al., 2001; Vidal and Ravichandran, 2005; Chetverikov and Péteri, 2005], dynamic NRT modeling, tracking and manipulation are new problems that have not been addressed before.

The fundamental contribution of this thesis is based on the observation that when a near-regular texture going through motion, its topological structure remains invariant, therefore, dynamic NRT can be modeled by a novel MRF with a wallpaper-groupbased lattice structure. Conventionally, dynamic texture analysis deals with stochastic textures [Szummer and Picard, 1996; Bar-Joseph et al., 2001; Wei and Levoy, 2000; Kwatra et al., 2003; Doretto et al., 2003; Bhat et al., 2004]. The problem of tracking

${ }^{1} \mathrm{~A}$ dynamic texture is modeled as a stationary process. A stationary process is a random process where all of its statistical properties do not vary with time [Papoulis and Pillai, 2002]. 

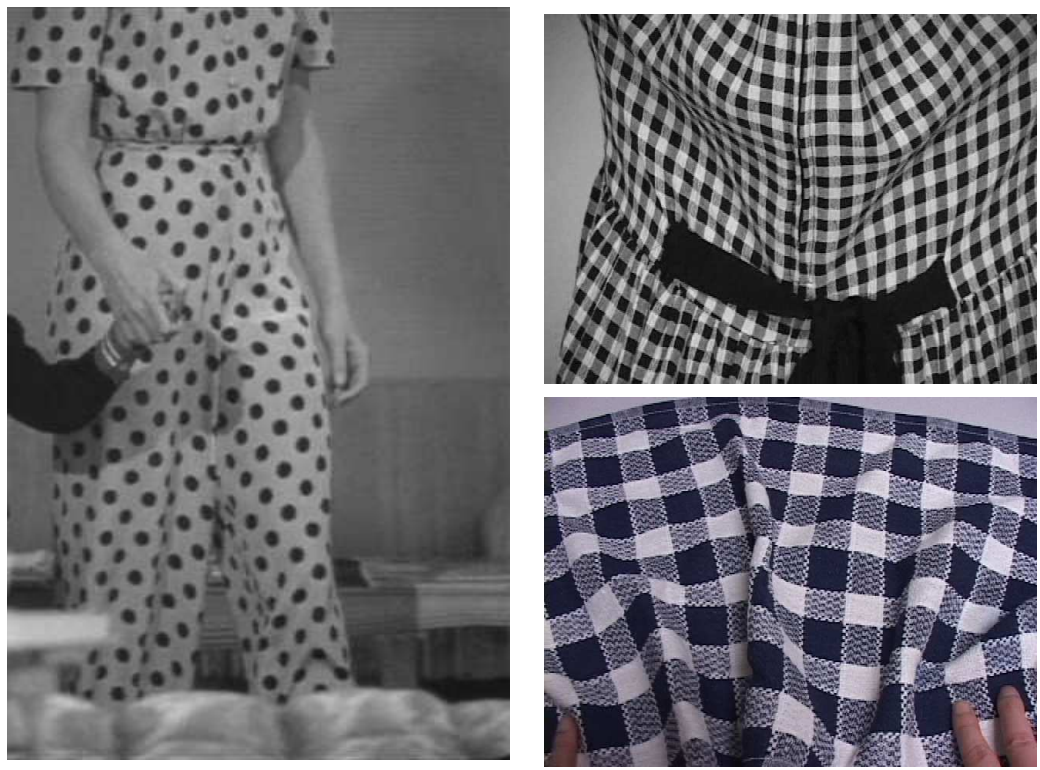

Figure 3.1. Examples of dynamic near-regular textures with tightly coupled textons. Textons are located on a deforming surface where there is no gap between neighboring textons.
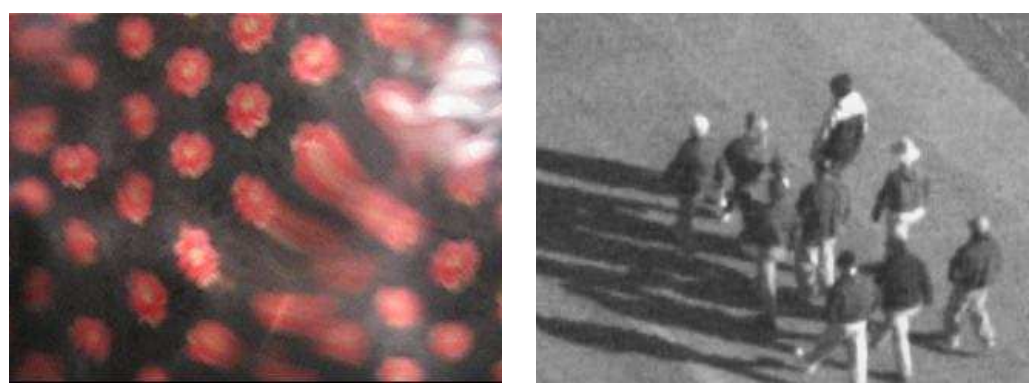

Figure 3.2. Examples of dynamic near-regular textures with loosely coupled textons. Neighboring textons are moved with loosely connected constraints. The texture at left is a pattern seen through disturbed water.

the motion of individual textons of a dynamic texture is undefined because there is no consensus on the definition of a texton of a stochastic texture [Zhu et al., 2005]. In existing work [Doretto et al., 2003; Wang and Zhu, 2004; Chetverikov and Péteri, 2005], a dynamic texture is usually treated as a statistical phenomena and a statistical model describing the collective motion is used in the analysis and synthesis process. The basic texture unit of a dynamic NRT is well defined since textons of an NRT can be characterized precisely based on their topological regularity. Thus, the analysis of a dynamic NRT can be carried out through a computationally feasible process of tracking the motion of individual textons of the dynamic NRT. Dynamic NRT tracking essentially provides a new way to analyze the regularity and randomness of a dynamic texture. 
Tightly coupled textons
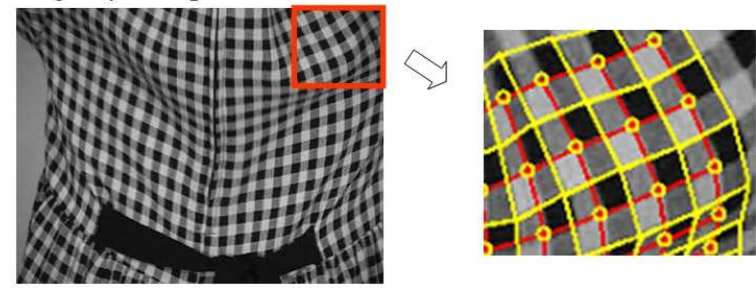

Loosely coupled textons
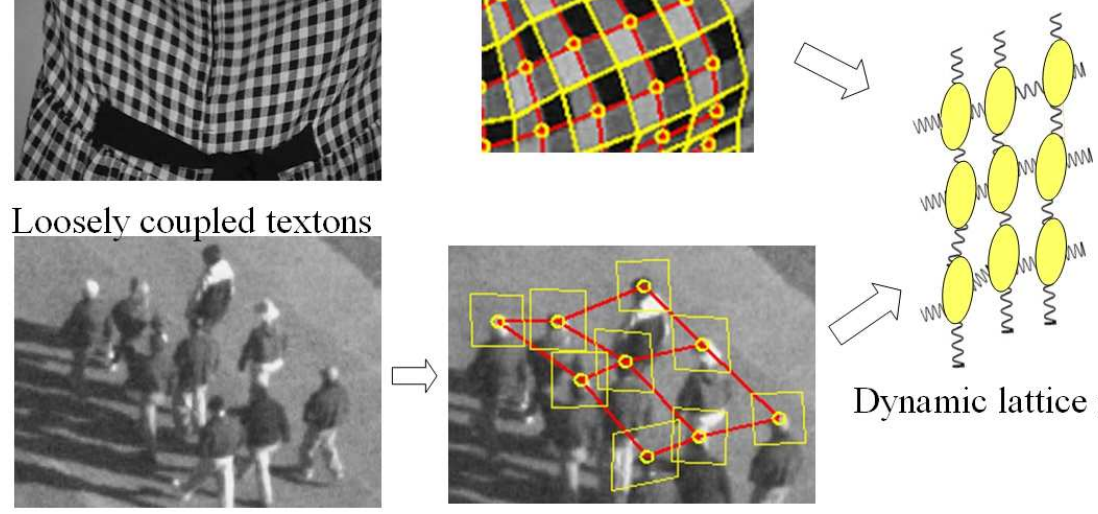

Dynamic lattice model

Figure 3.3. This figure shows the lattices (red lines) and textons (yellow quadrilaterals) of two types of NRTs: tightly and loosely coupled textons. We model the lattice of an NRT as a 2D MRF where each node represents a texton.

Tracking a dynamic NRT, however, poses several new computational challenges: the similar appearance of the textons of an NRT introduces a more severe ambiguous correspondence problem such that a tracking algorithm can easily mistake one texton for another. Furthermore, the tracking problem becomes very difficult when the textons of a dynamic NRT move rapidly or occlude each other on a folded surface. Due to these difficulties, tracking a dynamic NRT remained an unsolved problem.

This chapter is organized as follows: we first review related work on dynamic texture analysis and synthesis and visual tracking (section 3.1). We then define the scope of dynamic near-regular textures (section 3.2) and discuss the challenges of dynamic NRT tracking (section 3.3). We propose a lattice-based MRF model for representing dynamic NRT (section 3.4). Finally, we present our approaches for dynamic NRT tracking (section 3.5) and dynamic NRT manipulation (section 3.6).

\subsection{Related work}

Dynamic NRT tracking and manipulation are related to existing dynamic texture analysis and synthesis as they have similar goals: to model, analyze and reproduce a time-varying texture. Methodology-wise, dynamic NRT tracking is closely related to visual tracking. We review related work in respective fields in this section. 


\subsubsection{Dynamic Textures Analysis and Synthesis}

A dynamic texture or temporal texture is a sequence of image textures with motion [Nelson and Polana, 1992]. It exists in a 3D spatiotemporal volume with two spatial dimensions and one temporal dimension. Temporal textures are first coined by Nelson and Polana [1992]. They categorized visual motion into three classes: temporal textures, activities, and motion events. Temporal textures present statistical stationarity with indeterminate extent in space and time, such as fire, smoke or wavy water. Activities are motion patterns that are periodic in time and localized in space, such as walking or dancing. Motion events are single events that do not show periodicity in space or time, such as closing a door.

Dynamic Stochastic Texture. Since Nelson and Polana introduced the concept of temporal textures, most work in dynamic texture analysis and synthesis has mainly focused on dynamic stochastic textures [Szummer and Picard, 1996; Bar-Joseph et al., 2001; Wei and Levoy, 2000; Kwatra et al., 2003; Doretto et al., 2003; Bhat et al., 2004]. These algorithms assume that the motion is local and statistically stationary both in space and time. Both Szummer and Picard [1996] and Doretto et al. [2003] used an autoregressive model to model the motion in the video. While the former directly operates on the image pixels, the latter represents the input image sequence as a time series of filter response and constructs the model based on this time series. A survey on dynamic texture analysis can be found in [Chetverikov and Péteri, 2005].

In contrast to explicitly modeling the motion of a dynamic stochastic texture, there is another group of approaches [Wei and Levoy, 2000; Kwatra et al., 2003; Bhat et al., 2004; Kwatra et al., 2005] that do not use a motion model but directly synthesize a dynamic texture from the input data. The basic idea is similar to image-based texture synthesis (section 2.1); instead of combining 2D patches in the synthesis process, 3D cubes in the spatiotemporal domain are stitched to synthesize a dynamic texture. Wei and Levoy [2000] extended their 2D pixel-based synthesis algorithm to a 3D voxel-based synthesis algorithm without using any motion model. Kwatra et al. [2003] synthesized dynamic stochastic textures by stitching 3D spatiotemporal patches using the graph cut algorithm. 
One of the problems of dynamic texture synthesis is that the user cannot control synthesis results. This motivates several researchers to develop controllable synthesis algorithms. Bhat et al. [2004] presented an algorithm that allows the user to guide the texture synthesis process by specifying the flow of a dynamic fluid texture, such as fire, gas, smoke, or water. Temporal coherence and user control are addressed in this work. Recently, Kwatra et al. [2005] also proposed a synthesis algorithm that can be controlled by a flow field. They formulate the synthesis problem as an optimization problem that minimizes the texture energy and the controlling error. The texture energy is basically a MRF-based similarity metric that measures the appearance difference between the synthesized texture and the input texture in a statistical sense. The controlling error is measured based on the control criteria, e.g., the desired positions of texture elements set by the flow field. In addition to dynamic stochastic textures, their algorithm also works on some dynamic structural textures, e.g., keyboard texture. However, the regularity of NRT is not necessarily preserved by these algorithms.

Video Textures. Video texture [Schödl et al., 2000] treats image frames of a video as textures. The basic idea is to reshuffle and connect the sequence of video segments to produce an infinite length of video without visual obstruction. The video is generated from a video graph, which determines plausible transitions between different video segments. The synthesis approach of video textures is an extreme case of data-driven approach since it uses whole image frames as the basic unit without using a motion model or any modifications on image pixels. Video texture is good for synthesizing videos with activities but has limited control for synthesis results. The authors of video texture later improve their algorithm to handle the control problem and demonstrated its applications in animation [Schödl and Essa, 2002].

\subsubsection{Visual Tracking}

Our work is related to three types of tracking problems: deformable object tracking, cloth motion capture, and multi-target tracking. Image alignment is adopted in many deformable object tracking algorithms where different models are used to confine the deformation space, such as PCA [Cootes et al., 1998; Matthews and Baker, 2004], finite element mesh [Sclaroff and Isidoro, 1998], or subdivision surface [Guskov, 2004]. These 
models are not appropriate for tracking textons on a folded surface with occlusion because they assume the surface to be tracked is smooth and non-folded. Recently, Pilet et al. [2005] proposed a real-time non-rigid surface detection algorithm which tracks a non-rigid surface by repeatedly detecting and matching features in an image sequence. They combine a deformable mesh with a robust estimator to deal with large number of parameters, occluded features and erroneous matches. Features are detected using a classifier trained on a modal image. Feature matching between the input image and modal image are then performed through an optimization process which minimizes the correspondence error and non-smoothness. They do not handle NRT tracking in which repeated patterns cause a serious feature correspondence problem (section 3.3). Another related work in deformable object tracking is elastic bunch graph matching [Wiskott et al., 1997; Maurer and von der Malsburg, 1996] where a graph is used to model facial images. Each node of the graph corresponds to a facial landmark (e.g., nose, eye, mouth) and each edge is a $2 \mathrm{D}$ distance vector between two connected landmarks. Face tracking is achieved by iteratively matching image frames with a modal graph. They adopted a elastic distance measurement scheme in graph matching, which is similar to the concept of spring-network used in our MRF model. A big difference is that their graph is a deterministic model while ours is a statistical model. Also, the topology and the representation of image features in the elastic bunch graph (graph of irregular degrees, wavelet coefficients) are different from our MRF model (graph of degree 4, registration-based image observation model).

The goal of cloth motion capture is to capture the 3D motion of cloth. Special calibrated multi-camera systems [Scholz and Magnor, 2004; Scholz et al., 2005; Guskov et al., 2003; Pritchard and Heidrich, 2003], color-coded patterns [Scholz et al., 2005; Guskov et al., 2003], or controlled lighting [Scholz et al., 2005] are required. The special requirements on hardware and input patterns are used to reduce the tracking difficulties due to ambiguous feature correspondences or occlusion problems. Scholz et al. [2004] combines optical flow with geometric constraints (distance, curvature, contour) to track a synthetic motion of textured cloth under a calibrated multi-camera setting. Guskov [2002] developed an algorithm that can detect and track a black-white square pattern on cloth. His algorithm does not work on general NRT since only the 
image features of black-white square pattern are used in the detection and image alignment process. Our tracking algorithm can serve as the front end of a cloth motion capture system where no special purpose color-coded cloth pattern or lighting and camera calibration are required.

Tracking textons of a dynamic NRT can also be considered as a special case of multitarget tracking in which there are varying degrees of spatial constraints among different targets. The main difference between NRT tracking and multi-target tracking is that the topology among targets does not change in NRT tracking. Modeling the spatial relation among tracked targets using an MRF has been applied to ant tracking [Khan et al., 2004], sports player tracking [ $\mathrm{Yu}$ and $\mathrm{Wu}, 2005$ ] and hand tracking [Sudderth et al., 2004]. These algorithms may not track a dynamic NRT effectively since topology regularity is not explicitly modeled and utilized (see Figure 3.28 for an example).

Existing algorithms for deformable object tracking, cloth motion capture, or multitarget tracking succeed in their respective domains, but none of them deals with general NRT tracking problem under various types of motion, viewed through different media (water, air, ...) and occlusion conditions as treated in this paper. Our approach combines techniques used in multi-target tracking (MRF; section 3.4.2) and deformable object tracking (image alignment; section 3.4.2). Therefore, we can track various types of dynamic NRTs under different motion and conditions in a unified framework.

\subsubsection{Garment Motion Capture}

The output of the garment motion capture system developed by Scholz et al. [2005] is seemingly to be the most similar work to this thesis. We will briefly introduce their work and compare the difference between their approach and ours.

The garment motion capture system reconstructs the surface geometry of moving garment from multiple calibrated video cameras in a controlled lighting environment. Tracking by feature recognition is the main idea of this paper. They specially designed a cloth that have color-coded patterns, and use the coding information as the features to distinguish different part of cloth. A mesh representing the surface geometry to be captured is constructed using Alias's Maya software. This mesh construction also establishes the correspondence between mesh vertexes and color coded patterns. In the motion capture process, the system recognizes the color-coded features in videos from 


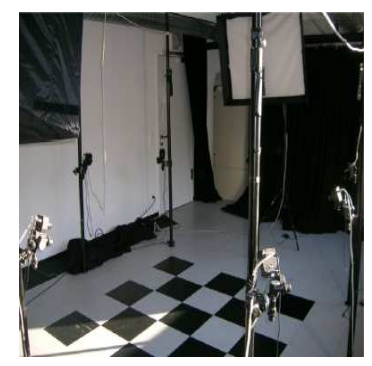

(a)

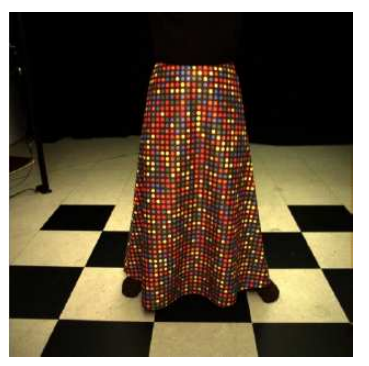

(b)

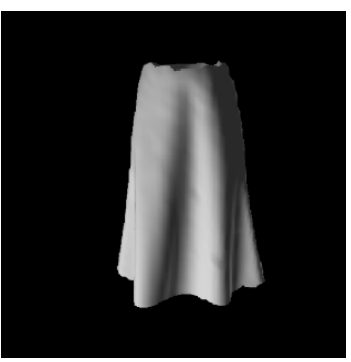

(c)

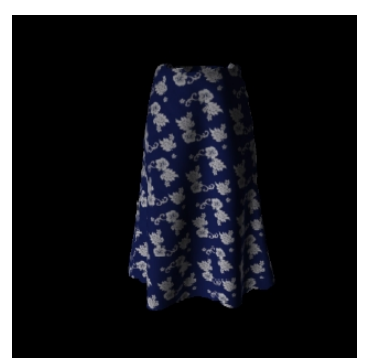

(d)

Figure 3.4. Garment motion capture system. (a) Camera setup: 8 cameras and 2 HMI lamps with softboxes. (b) A skirt with color-coded patterns used in the motion capture system. (c) Reconstructed surface. (d) Reconstructed surface with texture mapping. Pictures taken from [Scholz et al., 2005].

eight synchronized cameras. From color-coded features that are identified, the system reconstructs the $3 \mathrm{D}$ geometry of cloth. In the self-shadow or self-occluded region, the geometry are generated by the thin-plate interpolation. Once the surface geometry is obtained, any texture can be mapped to the reconstructed surface.

The fundamental difference between Scholz et al. approach and ours is that their goal is to capture the motion of cloth in a controlled environment while we focus on handling dynamic NRTs in the real-world. Their system is better suited for the applications in video studios or professional productions where special recording settings are not a major issue. Our approach, on the other hand, works under a more general and challenging conditions. We use monocular videos from an uncalibrated camera in normal environments, either indoors or outdoors. Our approach does not require the scene geometry to be known in advance. Finally, our approach does not use a specially designed color-coded cloth. Our approach can track general dynamic NRTs whether the texture is on a deforming surface or the texture is just a motion of multiple objects with near-regular spatial relation (see Figures 3.1 and 3.2 for examples).

\subsection{Introduction to Dynamic NRTs}

In this thesis, we consider dynamic NRTs whose motion may not be statistically stationary. Dynamic NRTs, originated from some regular textures [Liu and Tsin, 2002; Liu et al., 2004], exhibit topological regularity that may or may not preserve statistical stationarity. Figures 3.1 and 3.2 demonstrate several snapshots of dynamic NRT. One can observe that dynamic NRT exists in varied forms. It can be a fabric texture on 
moving cloth, a pattern seen through disturbed water, or even the motion of a crowd. Despite the varied forms of dynamic NRT, they have the following common properties:

\section{- Statistical appearance regularity}

Even though the gemetry and the appearance of individual textons in a dynamic NRT may vary, they bear strong similarity among themselves that can be considered as statistically similar, especially neighboring textons.

\section{- Topology invariance}

The topological structure of a dynamic near-regular texture remains invariant during motion, even though its geometry and appearance may vary from frame to frame.

A texton is considered to be the basic unit of a dynamic NRT. We can categorize dynamic NRTs into two types according to the spatial connectivity between textons: tightly coupled textons and loosely coupled textons. If the textons of a dynamic NRT are located on a deforming surface where there is no gap between textons, we call this type of texture a dynamic NRT with tightly coupled textons. Figure 3.1 shows three dynamic NRTs with tightly coupled textons. On the other hand, if two neighboring textons are allowed to move with a loosely connected constraint, there might be a gap or overlap between two neighbor textons. We call this type of near-regular texture a dynamic NRT with loosely coupled textons. Examples of dynamic NRT with loosely coupled textons include underwater pattern, or crowd motions, such as people in a crowd, a marching band, or a parade. Figure 3.2 shows two examples of loosely coupled textons. Figure 3.3 illustrates the lattice and textons of two dynamic NRTs where red lines represent the lattice and yellow quadrilaterals correspond to textons.

\subsection{Challenges of Tracking and Manipulating Dynamic NRTs}

Tracking and manipulating dynamic NRTs present new computational challenges due to some special properties of NRTs. These challenges include highly ambiguous texton correspondence, drastic temporal variation, occlusions, temporal coherence, and unknown camera parameters. The first three challenges are directly related to dynamic NRT tracking while the last two are related to dynamic NRT manipulation. 

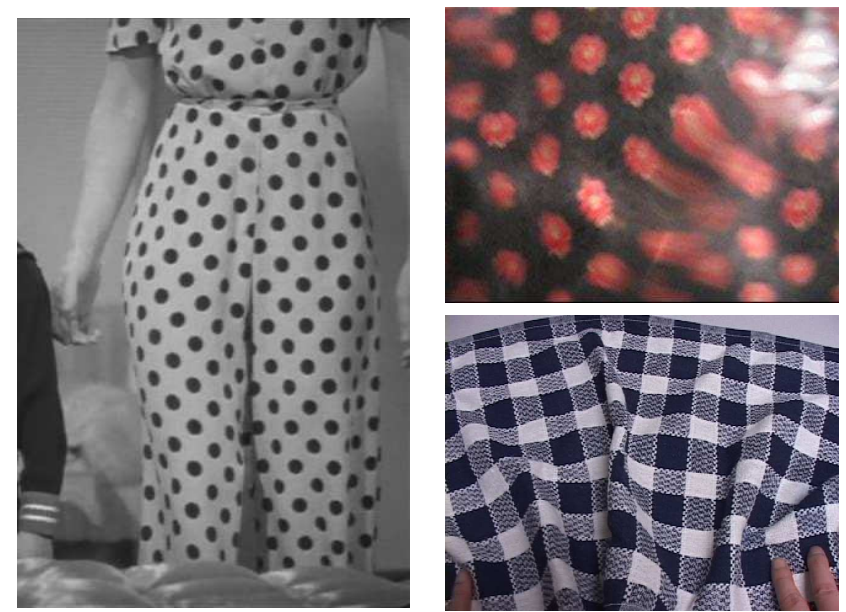

Figure 3.5. These images show the challenges of dynamic NRT tracking: ambiguous correspondences (left); temporal variations (top-right); occlusion (bottom-right).

\subsubsection{Ambiguous Texton Correspondences}

Maintaining the temporal correspondence of individual targets to be tracked is one of the most common tasks in multi-target tracking [Blackman and Popoli, 1999]. Ambiguous correspondences usually happen when two targets overlap or are within a close distance, thus a tracking algorithm can fail to follow their respective trajectories correctly. Ambiguous correspondences can be resolved by using the continuity of motion, or significant appearance difference of the two targets. If neither motion cue nor appearance cue is available, more sophisticated techniques, such as data association [Bar-Shalom, 1987; Cox, 1993], are needed to resolve the ambiguous correspondence problem.

The ambiguous correspondence problem in NRT tracking is caused by the strong appearance and geometry resemblance of NRT textons. The fabric pattern on the trousers in Figure 3.5 illustrates an example. Although textons of an NRT may have different appearance or geometry across a textured region due to variation of the surface geometry, the appearance of textons within a local region are similar. This causes a tracking algorithm to mistake one texton for another easily and lose the temporal correspondence of individual textons during tracking process. Ambiguous correspondences can also happen when an NRT is on a folding surface since textons can overlap in the folded region. Furthermore, spatial aliasing can also introduce ambiguous temporal correspondences. 
This occurs on a dynamic NRT moves rapidly such that the movement of a texton between two frames is larger than the size of a texton. The ambiguous correspondence problem is a challenge for NRT tracking because neither motion continuity nor appearance difference is helpful.

\subsubsection{Temporal Variations}

Although textons of an NRT have an underlying appearance and geometry regularity due to its regular texture origin, their appearance and geometry may vary dramatically when the texture undergos different types of motions. For example, if a texton is on a $3 \mathrm{D}$ surface, its image intensities change because the lighting condition varies as the surface geometry deforms, such as shading variations or shadowing effects. The shape of a texton also varies when a surface is deforming. An extreme case of geometry and appearance variations happen in the dynamic underwater texture, where textons are seen through disturbed water (Figure 3.5). Surface refraction and reflection cause the shape and appearance of a texton vary dramatically. Moreover, motion blur occurs when the water surface is disturbed. These cause additional challenges for the NRT tracking problem.

\subsubsection{Occlusions}

Occlusion is a common problem in all dynamic texture tracking. There are two types of occlusions: self occlusion and external. Figure 3.5 (bottom right) illustrates examples of both external and self occlusion problem. External occlusion happens when a texture is occluded by another object. It is easier to overcome the external occlusion problem if the appearance of an external object is substantially different from that of textons. The most difficult case occurs when an NRT has a self-occlusion. Simply relying on the appearance difference cannot resolve the foreground background separation problem because occluded textons have similar appearance. We need additional information, such as global structure, local geometric relation, or tracking history to solve this problem.

Another problem of NRT tracking is that textons might leave/enter the scene from boundary regions, it is important to detect these changes and disconnect/connect these textons to the current lattice topology accordingly. 


\subsubsection{Temporal Coherence}

Maintaining temporal coherence is very important for dynamic texture synthesis and manipulation because it is the principal criterion that the human uses to judge the quality of a synthesized or manipulated dynamic texture. In fact, it has been shown in psychophysical findings that the human visual system assumes objects move in consistent trajectories rather than abruptly changing their direction [Ramachandran and Anstis, 1983; Watamaniuk et al., 1995; Burgi et al., 2000]. Other studies of human visual system also show that human brains combine local estimation of velocity across space [Watamaniuk and Sekuler, 1992] and time [McKee and Welch, 1985] to predict and estimate motion through temporal coherence.

Maintaining temporal coherence in dynamic NRT replacement poses additional difficulties compared to dynamic texture synthesis since humans are especially sensitive to NRT consistency [Rao and Lohse, 1993]. Capturing the coherent geometric and lighting deformations temporally is required because the goal of replacement is to preserve the geometric and lighting deformation fields in the original texture. This means that we need to track the motion of individual textons so that a sequence of geometric and lighting DFs from an input dynamic NRT can be extracted. Indeed, dynamic texture replacement in videos has not been attempted previously because there is neither an effective algorithms to track textons of a dynamic texture nor an appropriate existing algorithm to handle the temporal coherence problem.

\subsubsection{Unknown Camera Parameters}

Real-world videos are usually recorded using an uncalibrated camera in an uncontrolled environments. This introduces additional challenges to texture tracking and manipulation. First, it is more difficult to deal with the occlusion problem caused by an oblique viewing angle since only a monocular video is available. Second, it is difficult to reconstruct the 3D geometry of a deforming surface in a single view video. Without knowing the surface geometry, simply texture mapping will not achieve realistic texture replacement. Third, uncontrolled environment causes unknown and unexpected illumination variation in tracking and also increases difficulties for capturing the lighting effects in a video. 


\subsection{Mathematical Model of Dynamic NRTs}

An effective computational model that respects and exploits the properties of the NRT is the key to the problems of dynamic NRT tracking and manipulation. We develop a lattice-based MRF model that integrates a high-level topological structure model and a low-level registration-based texton geometry and appearance model. Under such formalization, the proposed model characterizes the lattice topology and the geometry and appearance of individual textons of a dynamic NRT. Based on our lattice-based MRF model, we are able to solve the dynamic tracking problem under a statistical inference formulation and develop a dynamic NRT manipulation system to change or superimpose an augmented image on a dynamic NRT. NRT's unique properties create new challenges (section 3.3) for dynamic NRT tracking and at the same time provide important cues for solving the problem.

but they provide important cues for handling a dynamic NRT at the same time. In this section, we propose a lattice-based MRF model for dynamic NRT. Topology invariance and geometry regularity are exploited in the lattice structure model to resolve ambiguous correspondences and the occlusion problem. Geometry and appearance regularity are used to detect textons and deal with temporal variations.

\subsubsection{Texton Geometry Model}

A texton of a dynamic NRT is a tile that deforms in geometry and photometry with time (section 1.1). The texton geometry model defines the local appearance of an NRT. We build a statistical local transformation model for each texton. Specifically, the geometry of a quadrilateral texton is represented by two non-independent affine transformations (Figure 3.6). These two affine transformations map image pixels from a rectangular domain $[1, w] \times[1, h]$ to the quadrilateral region of the texton where $h=$ length $\left(\mathbf{t}_{\mathbf{2}}\right)$ and $w=$ length $\left(\mathbf{t}_{\mathbf{1}}\right)$. We call the image of a texton in its rectangular domain an aligned texton. Each of the two transformations is parameterized by the positions of three vertices of the quadrilateral as a $2 \mathrm{D}$ affine transformation has 6 free parameters. Let $\left(c^{i k x}, c^{i k y}\right)$ be the image coordinates of the $k$ th vertex of texton $i(k=1,2,3,4)$, the 


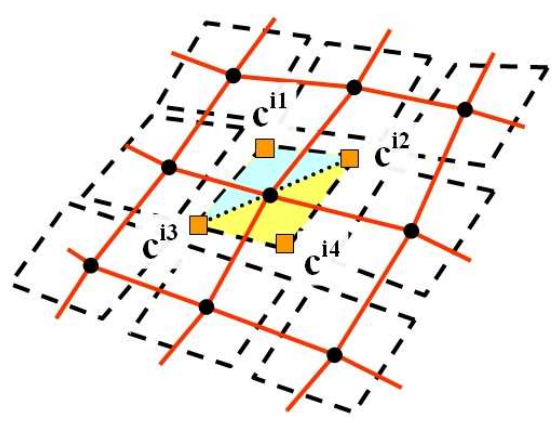

(a) loosely coupled textons

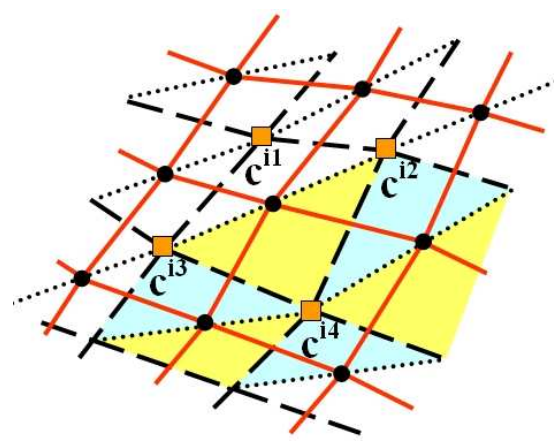

(b) tightly coupled textons

Figure 3.6. Texton geometric model. Red lines form the quadrilateral lattice structure of a dynamic NRT. $\mathbf{c}^{i 1}, \mathbf{c}^{i 2}, \mathbf{c}^{i 3}, \mathbf{c}^{i 4}$ denote image coordinates of the four vertices of the texton $i$. A texton is divided into two triangles (blue and yellow shaded), and the vertex coordinates of each triangle parameterize an affine transformation. (a) loosely coupled textons: the vertex positions of a texton are determined independently. (b) tightly coupled textons: the vertex positions of a texton are jointly determined by its neighboring textons (e.g., $\mathbf{c}^{i 4}$ is involved in six affine transformations).

two affine transformations representing the geometry of texton $i$ are parameterized as

$$
\begin{aligned}
\mathbf{A}^{\mathbf{i} 1} & =\left[\begin{array}{ccc}
c^{i 1 x} & c^{i 2 x} & c^{i 3 x} \\
c^{i 1 y} & c^{i 2 y} & c^{i 3 y} \\
1 & 1 & 1
\end{array}\right]\left[\begin{array}{ccc}
1 & w & 1 \\
1 & 1 & h \\
1 & 1 & 1
\end{array}\right]^{-1} \\
\mathbf{A}^{\mathbf{i} 2} & =\left[\begin{array}{ccc}
c^{i 2 x} & c^{i 3 x} & c^{i 4 x} \\
c^{i 2 y} & c^{i 3 y} & c^{i 4 y} \\
1 & 1 & 1
\end{array}\right]\left[\begin{array}{ccc}
w & 1 & w \\
1 & h & h \\
1 & 1 & 1
\end{array}\right]^{-1}
\end{aligned}
$$

These affine parameters (i.e., vertex positions) are determined through an image alignment process where each texton is registered to a texton template. A texton template is an aligned texton obtained in the first frame of the input video (section 3.5.1). Figure 3.7(b) shows an example of aligned textons in the first frame of a video. Each aligned texton in (b) serves as a texton template for the corresponding texton.

For loosely coupled textons, we compute the affine transformations of different textons independently. For tightly coupled textons, a constraint between neighboring textons need be enforced. This is done in a piecewise affine model where a texton vertex is used to parameterize multiple texton affine transformations who share the same texton (Appendix A.2). Figure 3.6 illustrates the geometric model of loosely and tightly coupled textons. 


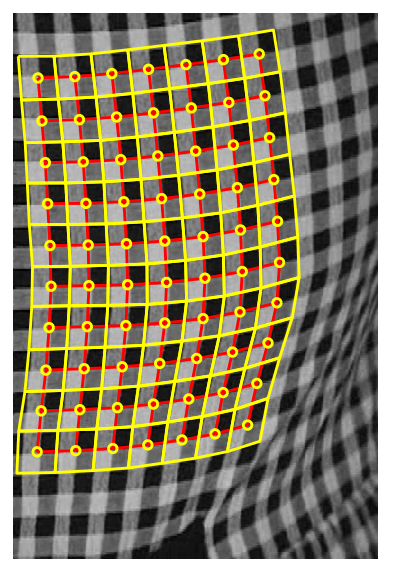

(a)

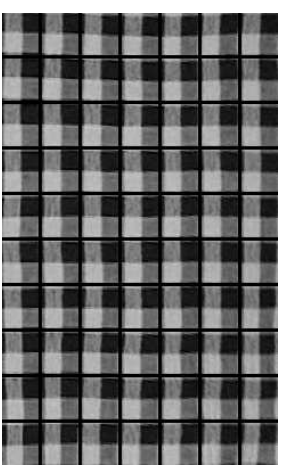

(b)

Figure 3.7. (a) The first frame of an dynamic NRT where a yellow quadrilateral represents a texton and red lines form the lattice. (b) Aligned textons based on textons in (a). These aligned textons serve as initial texton templates and are updated in successive frames.

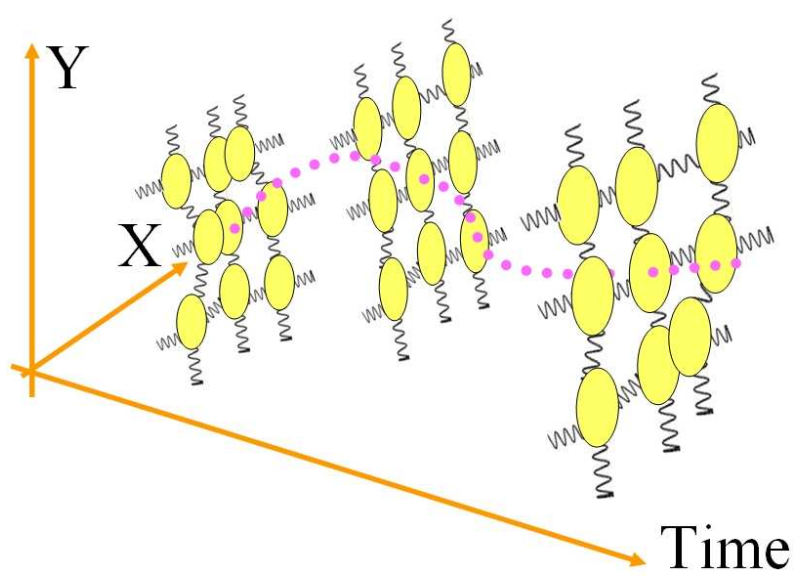

Figure 3.8. We view the lattice of a dynamic NRT as a network of springs that connect individual textons of a dynamic NRT. Yellow ovals represent textons.

\subsubsection{Lattice Structure Model of a Dynamic NRT}

The lattice $L$ of a dynamic NRT functions as the skeleton of a texture topologically. $L$ is a graph of degree 4 connecting all textons of an NRT. The root of a degree- 4 graph lattice comes from crystallographic group theory that all wallpaper patterns have a quadrilateral lattice characterizing its fundamental generating region. When an NRT moves, its lattice may deform accordingly, but its topology remains invariant. These properties resemble the behavior of a network of springs in which each spring controls the mutual distance between two textons locally. The network combines the forces from 
individual springs to maintain a global spatiotemporal structure (Figure 3.8). Our observation of the similarity between a lattice structure and a spring network is inspired by the physics-based cloth motion simulation where a spring-damper network is used to model the dynamics of cloth motion. The specific topology of our network, however, is soundly based on the mathematical theory of wallpaper groups [Fedorov, 1885; Grünbaum and Shephard, 1987][Liu et al., 2004 PAMI].

We can also view the lattice structure and the relation (or interaction) between textons from a statistical viewpoint that the probabilities of the states of textons are locally dependent. The lattice structure provides a well-defined neighborhood structure. The probability of the position of a texton is influenced more by those of neighboring textons than those of distant textons. The probability of the shape of a texton has similar properties to its neighbors. These Markov properties make Markov Random Field (MRF) a natural candidate to embed the global lattice structure of a dynamic NRT under a statistical framework.

The MRF model provides a systematic way to model context dependent entities such as image pixels and other spatially correlated features. The MRF model characterizes mutual influences among such entities using Markovian probabilities. The MRF model has been widely used in many applications [Li, 2001], from low-level processing, such as image segmentation and restoration, surface reconstruction, and edge detection to high-level vision, such as object matching and recognition. Although the MRF model has been applied to texture analysis and texture synthesis in the past, they are usually used for low-level processing, such as modeling the probabilities of pixel intensities. The effective combination of a global lattice structure with MRF enables us to capture the innate property of a dynamic NRT: globally and topologically regular while locally and appearance-wise (geometric and photometric) randomness.

An MRF is an undirected graph $(\mathcal{V}, \mathcal{E})$ where $\mathcal{V}$ and $\mathcal{E}$ denote the set of vertexes and edges in the graph respectively. Each vertex in the graph corresponds to a random variable. The joint probability of all random variables is factored as a product of local potential functions at each node, and the interactions are defined on neighborhood cliques represented by the connected edges in the graph. The most common form of 
MRF is a pairwise MRF in which the cliques are a pair of connected nodes in the undirected graph:

$$
p\left(x^{1}, x^{2}, \ldots, x^{N}\right) \propto \prod_{(i, j) \in \mathcal{E}} \varphi\left(x^{i}, x^{j}\right)
$$

where $x^{i}$ is a random variable representing the probability of node $i . \varphi\left(x^{i}, x^{j}\right)$ is the potential function between $x^{i}$ and $x^{j} . N$ is the total number of nodes in the graph. An intuitive way to understand the properties of an MRF is to consider it as a network of springs. The potential function is like a measurement of the spring energy between two connected nodes $x^{i}$ and $x^{j}$. If the potential function value is large, that means there is a strong bonding between $x^{i}$ and $x^{j}$ since their probabilities are highly dependent.

Following Equation (3.3), we can model the probability of a lattice configuration of an NRT using the following MRF,

$$
p\left(x^{1}, x^{2}, \ldots, x^{N}, z^{1}, z^{2}, \ldots, z^{N}\right) \propto \prod_{(i, j) \in \mathcal{E}} \varphi\left(x^{i}, x^{j}\right) \prod_{i=1}^{N} \phi\left(x^{i}, z^{i}\right)
$$

where $x^{i}$ and $z^{i}$ are random variables representing the state and image observation of texton $i$, respectively. The state of a texton is defined as $x^{i}=\left(c^{i 1 x}, c^{i 1 y}, c^{i 2 x}, c^{i 2 y}\right.$, $\left.c^{i 3 x}, c^{i 3 y}, c^{i 4 x}, c^{i 4 y}, v^{i}\right)$. The pair $\left(c^{i k x}, c^{i k y}\right)$ denotes the image coordinates of the $k$ th vertex $(k=1,2,3,4)$ of texton $i$ and $v^{i} \in(0,1)$ represents the visibility of texton $i$. Equation (3.4) is essentially a directly application of Equation (3.3) in that we introduce the image likelihood function $\phi\left(x^{i}, z^{i}\right)$ to associate the probabilistic relation between the state of textons and their image observations. The definitions of $\varphi\left(x^{i}, x^{j}\right)$ and $\phi\left(x^{i}, z^{i}\right)$ will be clear shortly.

The MRF defined in Equation (3.4) is also called Markov network ${ }^{2}$ [Freeman et al., 2000]. Let $X=\left(x^{1}, x^{2}, \ldots, x^{N}\right)$ and $Z=\left(z^{1}, z^{2}, \ldots, z^{N}\right)$ be the state of lattice configuration and the image observations of all textons, Equation (3.4) can be represented as a posterior probability ${ }^{3}$ :

$$
p(X \mid Z) \propto P(X, Z) \propto \prod_{(i, j) \in \mathcal{E}} \varphi\left(x^{i}, x^{j}\right) \prod_{i=1}^{N} \phi\left(x^{i}, z^{i}\right)
$$

where $\prod \varphi\left(x^{i}, x^{j}\right)$ is a lattice structure model and $\prod \phi\left(x^{i}, z^{i}\right)$ an image observation model. The lattice structure model captures the global structure of a lattice and resolves

${ }^{2}$ MRF and Markov network will be interchangeably used in this thesis.

${ }^{3} \mathrm{p}(\mathrm{Z})$ is a constant over $X$ 
ambiguous correspondences using the topological relation between neighboring textons. The image observation model integrates a texton geometry model (section 3.4.1) which aligns textons by minimizing image differences to handle local deformation of individual textons. In the following paragraphs, we will describe the potential function and the neighborhood configuration in our lattice structure model and define the image likelihood function in our image observation model.

Lattice Structure Model. The potential function in our MRF is defined as follows:

$$
\begin{gathered}
\varphi\left(x^{i}, x^{j}\right)=e^{-\beta \cdot d_{g}\left(x^{i}, x^{j}\right)} \\
d_{g}\left(x^{i}, x^{j}\right)=\left(\left\|\mathbf{c}_{m}^{i}-\mathbf{c}_{m}^{j}\right\|-l^{i j}\right)^{2} \cdot v^{i} v^{j}
\end{gathered}
$$

where $\beta$ is a global weighting scalar that is applied to all springs. $\beta$ weights the influence of the lattice model versus the observation model in the Markov network. $d_{g}$ is a function that measures the geometric deformation (spring energy function). $\mathbf{c}_{m}^{i} \in \mathbb{R}^{2 \times 1}$ is the mean position of four vertices of the texton $i$. This potential function acts like a spring that adjusts the position of textons based on their mutual distance. The rest length $l^{i j}$ of the spring is spatially dependent. To handle occlusion, $v^{i}$ and $v^{j}$ in Equation (3.7) are used to weigh the influence of a node by their visibility status.

The topology of the graph $\mathcal{E}$ defines how textons are related to each other in the lattice structure model. To model the global constraints and local variations of the probabilities of the states of textons properly, we use an analogy between an MRF and a network of springs. If graph $\mathcal{E}$ is a complete graph, each texton is connected with all other textons. Thus, the motion of a texton would be directly affected by all textons leading to overly constrained. On the other hand, if there are no edges in the graph, the global structure among textons reduces to isolated unconstraint textons.

We adopt a topology similar to the spring connection configuration used in [Provot, 1995; Choi and Ko, 2002] in the lattice structure model. It has been shown in cloth simulation that this kind of configuration provides a good balance between structural constraint and local deformations. Figure 3.9(a) shows this adopted spring configurations. We can convert this spring configuration into the topology of a graph. According to this graph, we can therefore define the neighborhood configuration of the MRF where the state of a node depends on the states of its twelve neighbors. Figure 3.9(b) shows 


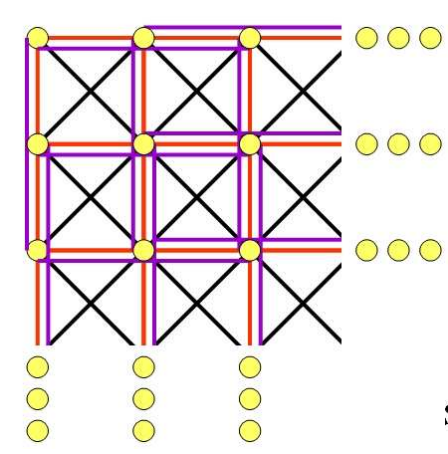

(a)

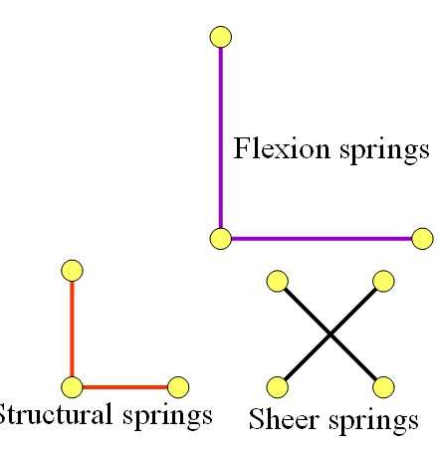

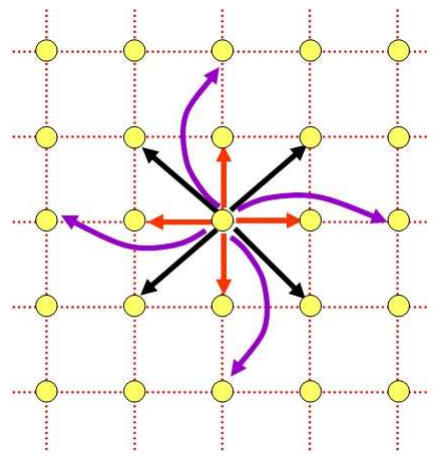

(b)

Figure 3.9. (a) The cloth spring configurations in [Provot, 1995]. The yellow circles and lines represent mass particles and and springs respectively. There are 1) structural springs (red lines) connecting nearest-neighbor particle along vertical and horizontal lines, 2) shear springs (black lines) connecting a particle's nearest-neighbor particles along diagonals, and 3) flexion springs (purple lines) connecting a particle with its second neighbor along vertical and horizontal lines. (b) neighborhood configuration of our MRF model: a node is connected to twelve neighbors.

the neighborhood configuration of our MRF. We also did an experiment to validate the 12-neighbor configuration is appropriate for our application (see section 3.5.6).

Image Observation Model. We define the image likelihood as follows:

$$
\phi\left(x^{i}, z^{i}\right) \propto e^{-\frac{1}{v^{i}} d_{a}\left(x^{i}, z^{i}, T^{i}\right)}
$$

where the appearance difference $d_{a}=\sum_{r=1}^{2} \sum_{\mathbf{p}}\left\|z^{i r}(\mathbf{p})-T^{i}(\mathbf{p})\right\|^{2}$ is the sum of squared differences (SSD) between a texton template $T^{i}$ and the observed texton. $\mathbf{p}$ denotes a pixel location in the coordinate frame of the template. $d_{a}$ is weighted by the visibility of a texton so that visible textons contribute more in the likelihood function. $z^{i r}=I\left(\mathbf{W}\left(\mathbf{p} ; \tilde{\mathbf{a}}^{i r}\right)\right)$ is an aligned texton obtained from the affine warp $\mathbf{W}$ whose parameter $\tilde{\mathbf{a}}^{i r}$ is computed by the Lucas-Kanade algorithm using the texton vertex coordinates $\left(c^{i k x}, c^{i k y}\right)$ as the initial values. Note that a quadrilateral texton is divided into two triangles and the vertex coordinates of each triangle are used to parameterize $\mathbf{a}^{i 1}$ and $\mathbf{a}^{i 2}$ respectively. If textons are tightly coupled, the textured region is modeled as a piecewise affine warp and the position of each texton vertex is affected by four neighboring textons. This enforces hard connected constraints among textons when computing $\tilde{\mathbf{a}}^{i r}$. If the textons are loosely coupled, $\tilde{\mathbf{a}}^{i r}$ of each texton is computed independently. This 
allows the observation model to handle more flexible motion, such as underwater texture, or people in a crowd.

Visibility Computation. The visibility of a texton is determined by constraints and measurements related to geometry and appearance of a texton. The constraints, which include topology, side length and area difference with neighboring textons, are used to decide whether a texton is valid and can be included in the tracking process. The measurements define the visibility score $v^{i}$ of a valid texton $i$ :

$$
v^{i}=\frac{1}{1+\rho}\left(\frac{s^{i}}{s^{*}}+\frac{\rho}{4} \sum_{k=1}^{4} \frac{\left|b_{k}^{i}-b_{k}^{*}\right|}{b_{k}^{*}}\right)
$$

where $s^{i}$ and $s^{*}$ are the area of texton $i$ and the seed texton. $b_{k}^{i}$ and $b_{k}^{*}$ are the $k$ th side length of texton $i$ and the seed texton. $\rho$ is a constant to weight the influence of area and side length variations in the visibility measurement. A visibility map $V$ is constructed based on the visibility scores of all textons:

$$
V=\left\{M^{i} \mid M^{i} \in(0,1), i=\ldots N\right\}
$$

where $M^{i}=1$ if $v^{i} \geq 0.5 ; M^{i}=0$ if $v^{i}<0.5$.

\subsubsection{Spatial Tracking-Texton Detection}

The appearance and geometry regularity of an NRT can be exploited by detecting all textons spatially. We consider an NRT as being formed by translating a texton on a plane where the shape and image intensities of the texton may vary across the plane. The texton detection and connection can be viewed as a tracking problem, more precisely, a spatial tracking problem. That is, each texton can be treated as a target to be tracked, and the trajectories of all texton centers form a lattice.

A difference between the spatial tracking and the temporal tracking is that spatial tracking can proceed in any order since all information is present simultaneously. Therefore, we can "grow" the lattice from regions where textons are more regular and reliable by propagating the lattice spatially to regions where textons are distorted or occluded.

The varying geometry regularity of an NRT can be used to predict the shape and position of a new texton. As described in section 3.4.1, the shape and position of a texton is represented by a pair of affine transformations. Assuming the geometric variations of textons are smooth locally, the shape and position of a new texton can be estimated from 
those neighboring textons. We can compute the affine transformation matrix of a new texton by linearly extrapolating from those of the neighboring textons. We can further refine affine parameters through an image alignment process. The texton geometry model (section 3.4.1) is used to confine the transformation space in the image alignment process by computing the affine parameters of all detected textons simultaneously. This makes the image alignment process more robust since it is a global adjustment.

\subsubsection{Temporal Lattice-based MRF model}

So far, the lattice-based MRF model provides the probabilities of lattice configurations at a single time instance. To incorporate the temporal variations of the MRF model for dynamic NRTs, these probabilities need to be propagated temporally. We formulate the temporal lattice-based MRF model of a dynamic NRT at frame $t$ as:

$$
p\left(X_{t} \mid Z_{t}\right) \propto \prod_{(i, j) \in \mathcal{E}} \varphi\left(x_{t}^{i}, x_{t}^{j}\right) \prod_{i=1}^{N} \phi\left(x_{t}^{i}, z_{t}^{i}\right)
$$

where $X_{t}=\left(x_{t}^{1}, x_{t}^{2}, \ldots, x_{t}^{N}\right)$ is the configuration of the lattice at frame $\mathrm{t}$ and $Z_{t}=$ $\left(z_{t}^{1}, z_{t}^{2}, \ldots, z_{t}^{N}\right)$ is the image observation at frame $t . z_{t}^{i}$ represent the state and the image intensities of texton $i$ at frame $t . x_{t}^{i}=\left(c_{t}^{i 1 x}, c_{t}^{i 1 y}, c_{t}^{i 2 x}, c_{t}^{i 2 y}, c_{t}^{i 3 x}, c_{t}^{i 3 y}, c_{t}^{i 4 x}, c_{t}^{i 4 y}, v_{t}^{i}\right)$ is the state of a texton. The pair $\left(c_{t}^{i k x}, c_{t}^{i k y}\right)$ denotes the image coordinates of the $k$ th vertex of the texton $(k=1,2,3,4)$ and $v_{t}^{i} \in(0,1)$ represents visibility of texton $i$ at frame $t$. Figure 3.10 illustrates our temporal lattice-based MRF model.

The potential function $\varphi\left(x_{t}^{i}, x_{t}^{j}\right)$ and image likelihood function $\phi\left(x_{t}^{i}, z_{t}^{i}\right)$ in Equation (3.11) are defined respectively,

$$
\begin{gathered}
\varphi\left(x_{t}^{i}, x_{t}^{j}\right)=e^{-\beta \cdot d_{g}\left(x_{t}^{i}, x_{t}^{j}\right)} \\
d_{g}\left(x_{t}^{i}, x_{t}^{j}\right)=\left(\left\|\mathbf{c}_{m}^{i}-\mathbf{c}_{m}^{j}\right\|-l_{t}^{i j}\right)^{2} \cdot v_{t}^{i} v_{t}^{j} \\
\phi\left(x_{t}^{i}, z_{t}^{i}\right) \propto e^{-\frac{1}{v_{t}^{i}} d_{a}\left(x_{t}^{i}, z_{t}^{i}, T_{t}^{i}\right)}
\end{gathered}
$$

where all notations follow the same definition in Equation (3.6) - Equation (3.14) but with additional time index.

To handle the temporal variation of lattice structure, the rest length $l_{t}^{i j}$ of the spring is not only spatially dependent but also temporally adaptive. We use an exponentially 


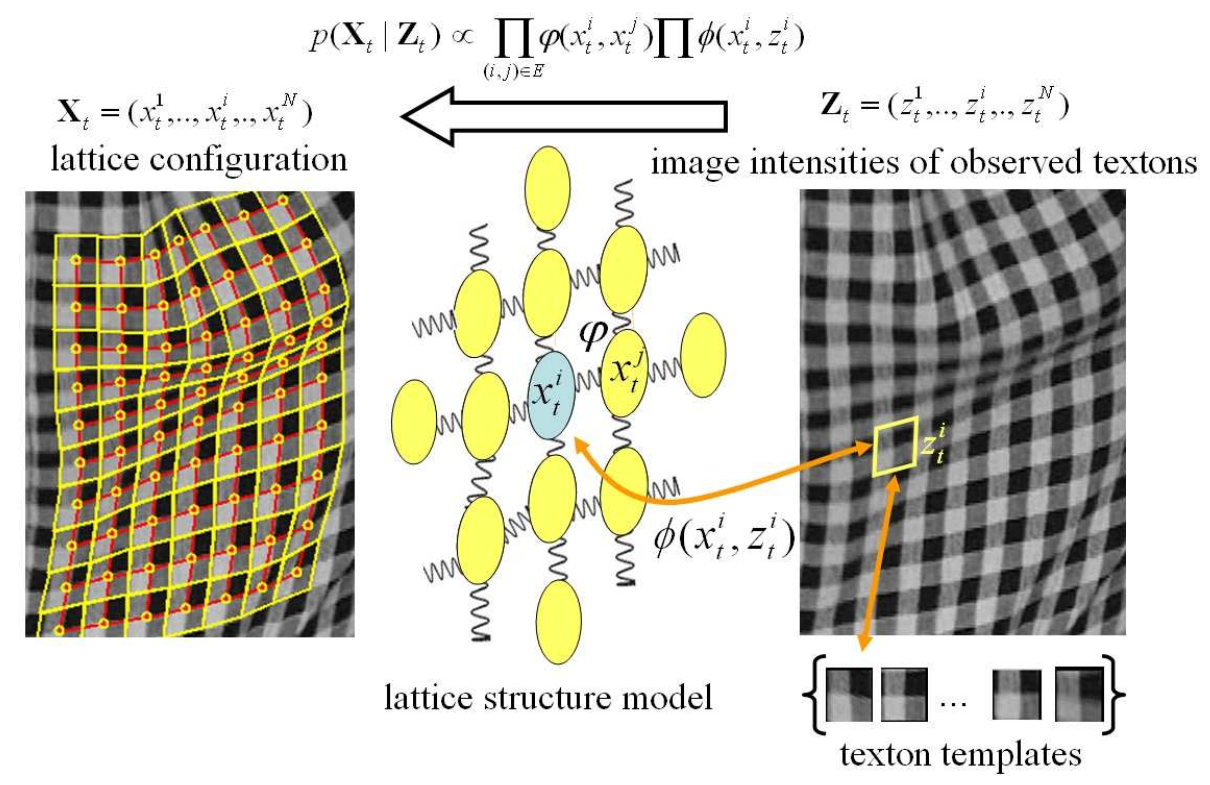

Figure 3.10. Illustration of our temporal lattice-based MRF model

decay function to model the temporal variation of the rest length of springs.

$$
l_{t}^{i j}=\frac{\sum_{f=1}^{\infty} l_{t-f}^{i j} e^{-\gamma f}}{\sum_{f=1}^{\infty} e^{-\gamma f}}
$$

where $\gamma$ is a parameter that controls how fast the exponential function decays.

\subsection{Dynamic NRT Tracking}

We develop a dynamic NRT tracking algorithm based on the proposed lattice-based MRF model. Our tracking algorithm consists of four components: 1) texton detection, 2) spatial inference, 3) temporal tracking, and 4) template update. An overview of our algorithm is shown in Figure 3.11.

In the initialization stage, the texton detection algorithm finds all textons in the first frame based on a single texton. All detected textons are then geometrically aligned. We call these aligned textons texton templates. A quadrilateral lattice is constructed by connecting the centers of detected textons.

In the tracking stage, texton detection is performed at each frame to include any additional texton entering the scene and removing the ones leaving the scene. We handle the texton tracking problem through a statistical inference process consisting of spatial inference and temporal tracking. The states of a texton (position, shape, and visibility) are sampled and its distribution is modeled by a particle filter in the tracking process. 
Initialization

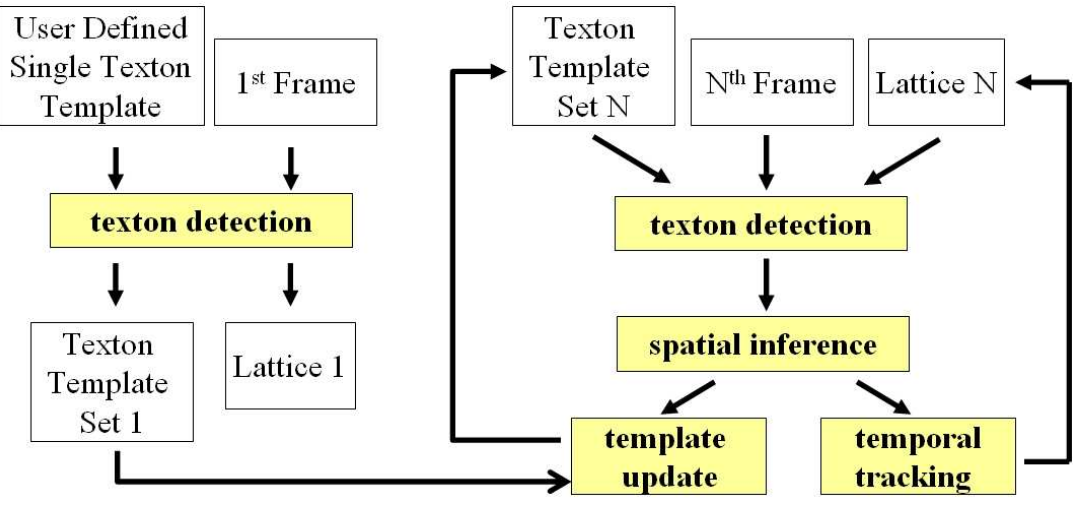

Figure 3.11. Approach overview

In each frame, a set of sampled states is drawn and a dynamic model generates the predicted states for the next frame. Belief propagation (BP) [Pearl, 1998; Yedidia et al., 2001; Freeman et al., 2000] is then applied to these predicted states to find the most likely lattice configuration based on the lattice structure model and image data. BP also provides the probability of each texton state, which is used to refine the approximation of the distribution of texton states through particle filtering. The above process iterates until the whole image sequence is tracked. In addition, the texton template set is updated to handle the variation of image intensities of textons in the tracking process.

\subsubsection{Tracking Initialization and Texton Detection}

In the initialization stage, the user identifies a single texton in the first image frame by specifying two vectors $\mathbf{t}_{1}$ and $\mathbf{t}_{\mathbf{2}}$ (three points) that form a parallelogram (Figure 3.12(a)). A texton template $T$ is constructed by transforming the parallelogram region in the image to a rectangular region $[1, w] \times[1, h]$, where $w=\operatorname{length}\left(\mathbf{t}_{\mathbf{1}}\right), h=\operatorname{length}\left(\mathbf{t}_{\mathbf{2}}\right)$, and the affine transformation matrix $\mathbf{A}_{1}$ is parameterized by the image coordinates of texton vertices $\left(c^{1 x}, c^{1 y}\right),\left(c^{2 x}, c^{2 y}\right),\left(c^{3 x}, c^{3 y}\right),\left(c^{4 x}, c^{4 y}\right)$,

$$
\mathbf{A}_{\mathbf{1}}=\left[\begin{array}{ccc}
c^{1 x} & c^{3 x} & \frac{c^{2 x}+c^{4 x}}{2} \\
c^{1 y} & c^{3 y} & \frac{c^{2 y}+c^{4 y}}{2} \\
1 & 1 & 1
\end{array}\right]\left[\begin{array}{ccc}
w & 1 & w \\
1 & h & \frac{h}{2} \\
1 & 1 & 1
\end{array}\right]^{-1}
$$

Once the first texton is identified, the second, third and fourth texton are obtained by translating the first texton by $\mathbf{t}_{1},-\mathbf{t}_{1}$ and $\mathbf{t}_{2}$. Using the first four textons as the basis for the initial lattice, the lattice grows by repeating the spatial prediction and the 


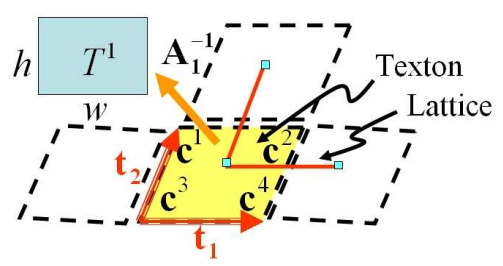

(a)

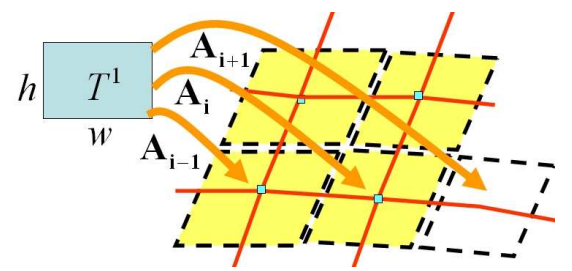

(b)

Figure 3.12. (a) Initial texton (yellow parallelogram formed by $\mathbf{t}_{\mathbf{1}}$ and $\mathbf{t}_{\mathbf{2}}$ ) and lattice (red lines). The neighboring textons are estimated by translating the first texton by $\mathbf{t}_{1},-\mathbf{t}_{\mathbf{1}}$, and $\mathbf{t}_{\mathbf{2}}$. (b) Spatial prediction of the position of a new texton.

validation steps. In the spatial prediction step, the vertices of a texton are estimated from existing textons. Let $\mathbf{A}_{i}$ be the affine transformation matrix that maps pixels of the texton template $T^{i}$ to the texton $i$ in the image. Suppose texton $i-1, i$, and $i+1$ are on the same lattice row or column, and $\mathbf{A}_{i-1}$ and $\mathbf{A}_{i}$ are known, $\mathbf{A}_{i+1}$ is predicted by $\mathbf{A}_{i+1}=\left(\mathbf{A}_{i} \cdot \mathbf{A}_{i-1}^{-1}\right) \cdot \mathbf{A}_{i}$. This equation can be interpreted as first mapping the texton template from the origin to the image region of the texton $i$, and then mapping the pixels from texton $i$ to $i+1$ by $\mathbf{A}_{i} \mathbf{A}_{i-1}^{-1}$. Here we assume the relative transformation from texton $i$ to $i+1$ can be approximated by the relative transformation from texton $i-1$ to $i$, i.e., $\mathbf{A}_{i} \mathbf{A}_{i-1}^{-1}$.

In the validation step, we verify if the predicted texton is valid by checking its associative topology constraints, and its area and side length difference with the neighboring textons. Additionally, the vertex positions of all valid textons are refined through an image alignment process where a global optimization that involves the whole lattice is performed (see Appendix A.2). The spatial prediction and validation steps are repeated until no new texton is detected. A texton template set $\mathcal{T}_{1}=\left\{T_{1}^{i}\right\}_{i=1}^{N}$ is constructed by collecting all valid texton template $T_{t}^{i}$, where $\mathcal{T}_{t}$ denotes the template set at frame $t$. The initial configuration of lattice is obtained by connecting all the centers of textons.

\subsubsection{Spatial Inference}

The temporal lattice-based MRF model (Equation (3.11)) describes the posterior probabilities of the lattice configuration of a dynamic NRT given an image observation of the NRT at frame $t$. Solving Equation (3.11) can be considered as a spatial inference problem where the most likely configuration of the lattice is inferred from the image 
observation of an NRT and the lattice structure model. We can apply the belief propagation algorithm (BP) [Pearl, 1998; Yedidia et al., 2001; Freeman et al., 2000] to solve this inference problem.

$\mathrm{BP}$ is an iterative algorithm for computing marginal distributions of random variables on a graphical model, such as MRF, Bayesian network, and factor graph. The BP algorithm introduces variables such as $m_{i j}\left(x_{t}^{j}\right)$ to propagate the marginal distribution among nodes. $m_{i j}\left(x_{t}^{j}\right)$ is a vector of the same dimensionality as $x_{t}^{j}$. Intuitively, $m_{i j}\left(x_{t}^{j}\right)$ can be interpreted as a messages passing from hidden node $i$ to hidden node $j$ about what state node $j$ should be. Messages are computed iteratively using the following update rule:

$$
m_{i j}\left(x_{t}^{j}\right) \leftarrow \sum_{x_{t}^{i}} \phi\left(x_{t}^{i}, z_{t}^{i}\right) \varphi\left(x_{t}^{i}, x_{t}^{j}\right) \prod_{k \in \mathcal{N}(i) \backslash j} m_{k i}\left(x_{t}^{i}\right)
$$

where $\mathcal{N}(i)$ denotes the neighbors of nodes $i$.

The marginal distribution of $x_{t}^{i}$, which is called the belief at node $i$, is proportional to the product of the local evidence at that node $\left(\phi\left(x_{t}^{i}, z_{t}^{i}\right)\right)$, and all messages coming into node $i$. By iteratively computing Equation (3.17), the marginal distribution at each node can be obtained using

$$
p\left(x_{t}^{i}\right)=\frac{1}{Q} \phi\left(x_{t}^{i}, z_{t}^{i}\right) \prod_{j \in \mathcal{N}(i) \backslash j} m_{j i}\left(x_{t}^{i}\right)
$$

where $Q$ is a constant for normalization. It has been shown that BP converges to an exact inference solution if the graph is a tree structure [Pearl, 1998] and an approximated inference solution if the graph contains loops [Weiss and Freeman, 2001]. For more details about belief propagation, please see [Yedidia et al., 2001].

Since the conventional BP algorithm works on discrete variables while the configuration of a lattice is described by continuous variables, we need to either discretize the state variables or apply continuous BP algorithms [Sudderth et al., 2003; Isard, 2003]. For computational efficiency, we choose to use the discrete BP and adopt the samplebased statistics to represent the continuous state variables for each texton. Particle filtering [Isard and Blake, 1998; Doucet et al., 2001] is applied to update the particle set for each texton in the temporal tracking process. 


\subsubsection{Temporal Tracking}

The spatial inference results provide the probabilities of lattice configurations at a single time instance. To track a lattice, these probabilities need be propagated temporally. This poses the temporal tracking problem as a sequential inference problem. Kalman filter [Kalman, 1960] is one of the common methods for this purpose; however, Kalman filter is not appropriate for our application since it does not deal with a nonGaussian or nonlinear random process. Although there are other variants of Kalman filter, such as extended Kalman filters [Anderson and Moore, 1979] and mixture of Gaussian approximations, they do not take into account all the salient statistical characteristics of random processes under consideration. Therefore, these methods usually lead to poor results for a non-Gaussian or nonlinear random process.

A general approach to handle non-Gaussian and nonlinear probability distributions in sequential inference is particle filters [Doucet et al., 2001]. Particle filtering is flexible in that it does not require any assumptions about the probability distributions of the data. It approximates the posteriori distribution by a set of particles where each particle is weighted by an observation likelihood and is propagated according to a dynamic model. We therefore apply particle filtering to solve the temporal tracking problem due to its flexibility and easiness in implementation.

The distribution of the lattice configurations in 3D spatiotemporal space are represented and maintained by particle filtering in temporal tracking. The belief distribution computed by the BP is used in importance sampling to draw new samples. The dynamic model is then applied to predict a set of states for each texton, and the discrete $\mathrm{BP}$ is applied to infer the most likely configuration based on these predicted states.

We use a second-order dynamic model, i.e., the current state of the lattice depends on the previous two states:

$$
p\left(X_{t} \mid X_{t-1}, X_{t-2}\right) \propto \prod p\left(x_{t}^{i} \mid x_{t-1}^{i}, x_{t-2}^{i}\right)
$$

where a constant velocity model with gaussian noise is used for the dynamic model for each texton:

$$
p\left(x_{t}^{i} \mid x_{t-1}^{i}, x_{t-2}^{i}\right)=\mathcal{N}\left(x_{t}^{i}-2 x_{t-1}^{i}+x_{t-2}^{i} ; 0, \Lambda_{i}\right)
$$

$\Lambda_{i}$ is a diagonal matrix whose diagonal terms correspond to the variance of the state at different dimensions. 
Our approach of combining BP and particle filter is similar to PAMPAS [Isard, 2003] in spirit, however, PAMPAS incorporates particle filter in the message propagation process within BP while we only use particle filter to carry the texton states between image frames. Note that Guskov et al. [Guskov et al., 2003] also used the Markov network to associate color-coded quadrilaterals in an image with the quadrilaterals of the surface model. They did not use the Markov network to infer the position and the shape of the textons.

\subsubsection{Template Update}

Since the appearance of textons vary during tracking process, it is necessary to update the texton template set. We use the template updating algorithm in [Matthews et al., 2004] where the basic idea is to correct the drift in each frame by additionally aligning the current image with the template at the first frame using the Lucas-Kanade algorithm. After aligning the current image with the previous frame, the computed warping parameters are used as the initial values in the additional alignment process. If the warping parameters obtained from the second image alignment process is close to the first one, the template is updated; otherwise, the template remains unchanged.

\subsubsection{Results}

Spatial Tracking Results. Our texton detection algorithm (section 3.5.1) can be used to initialize tracking as well as to extract the lattice of a static NRT [Liu et al., 2004] with minimal user input. Our algorithm greatly reduces the required user intervention in lattice extraction compared to our previous approach in section 2.2. This will facilitate the static NRT analysis process.

Figure 3.13 shows three texton detection results on two flat carpets and a fabric with perspective distortion and minor geometric deformation. Figure 3.14 shows another texton detection result where large deformations on the left and right boundaries of the dress. These results show that our detection algorithm can detect textons that are on flat and deformed surfaces with perspective distortions.

Tracking Dynamic NRTs without Occlusion. We tested our algorithm on several dynamic NRTs (Figure 3.15-3.18) under different types of motions. We also compared our algorithm against the robust optical flow algorithm [Black and Anandan, 1996] and 

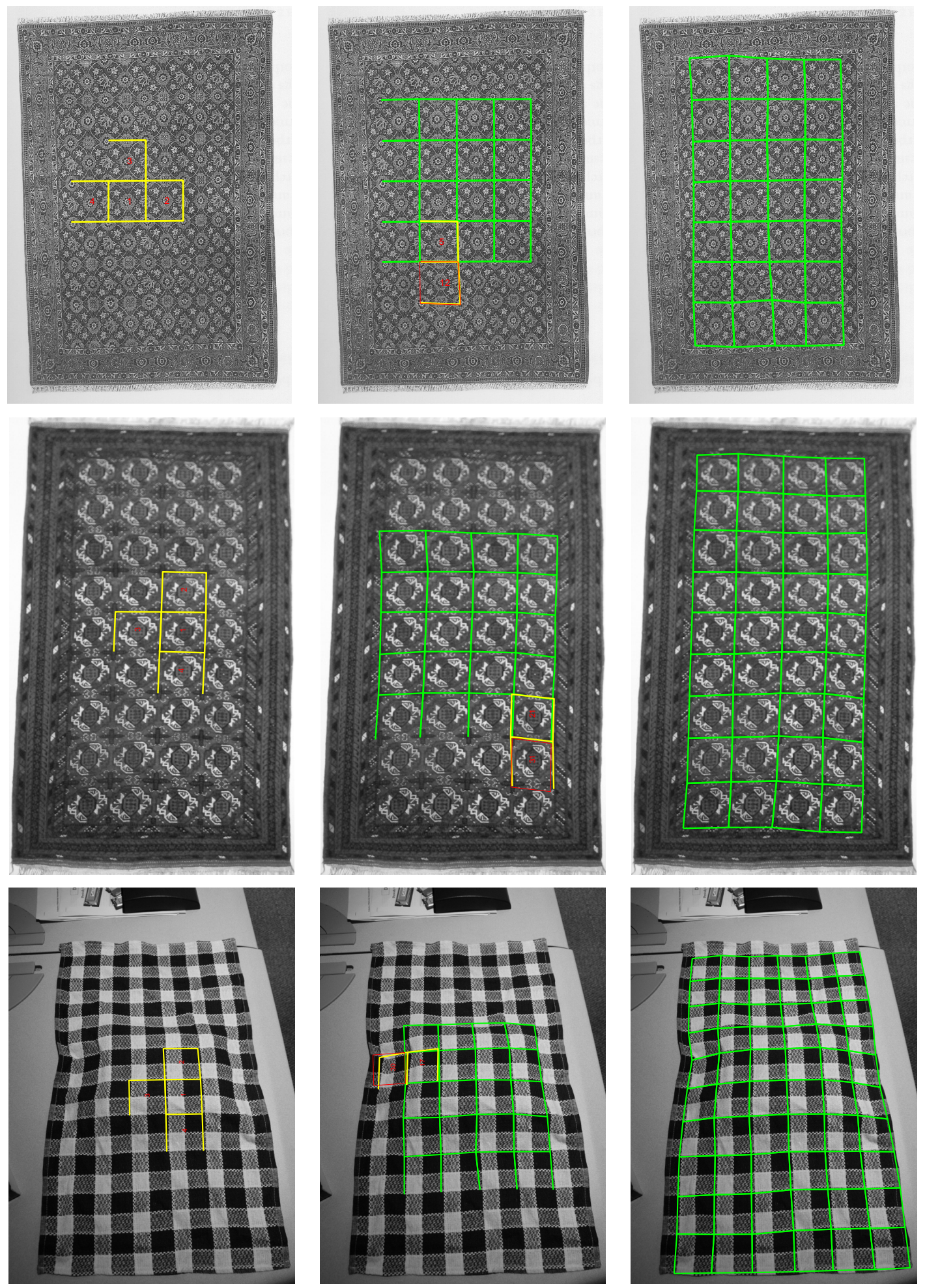

Figure 3.13. Texton detection results by spatial tracking. Left: initial lattice. Center: intermediate result. Right: final result. 


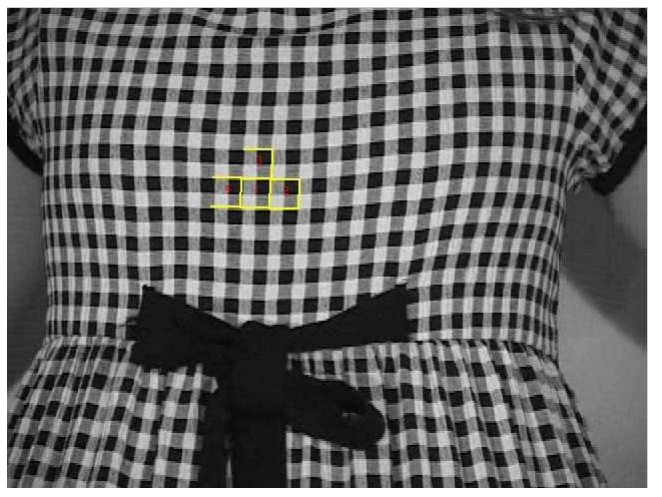

(a)

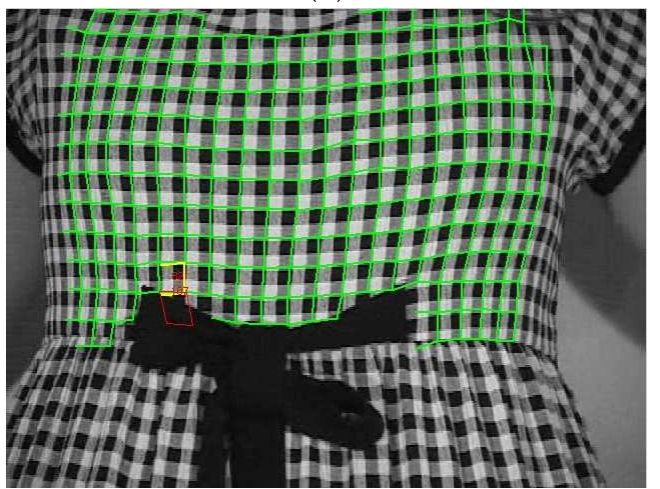

(c)

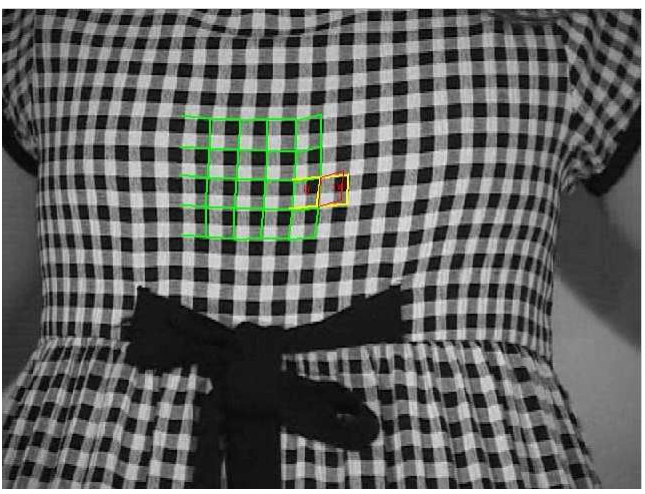

(b)

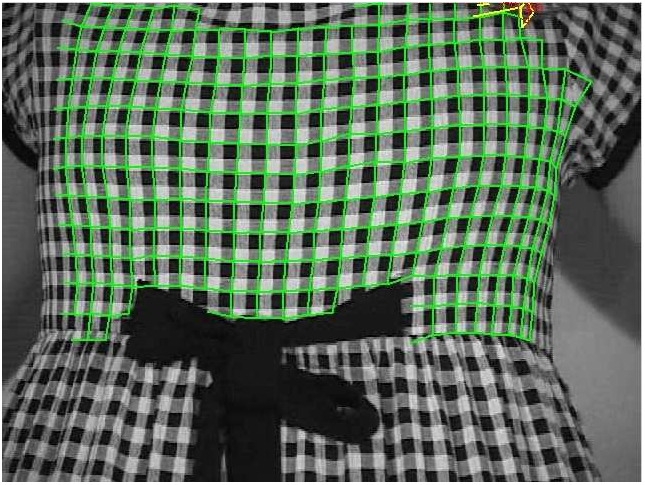

(d)

Figure 3.14. Texton detection results by spatial tracking (a) Initial lattice. (b)(c) Intermediate results. (d) Final result.

the Lucas-Kanade algorithm [Lucas and Kanade, 1981; Baker and Matthews, 2004]. We chose these two algorithms as base-line comparison because they are popular general purpose tracking algorithms. We tried different values of regularization term in the robust optical flow algorithm, and found that the value of 2.5 achieves the best tracking performance in our experiments. For Lucas-Kanade algorithm, 2D affine transformation is used in the tracking process.

Figure 3.15 shows tracking results of an NRT on slowly varying cloth. Due to the ambiguous correspondence challenge of NRT textons, both the robust optical flow algorithm and the Lucas-Kanade algorithm were distracted by neighboring textons (Figure 3.15 (a)(b)). We also tested our algorithm on dynamic NRTs with highly dynamic motion where a pattern was viewed through disturbed water. Figures 3.16 and 3.17 show the tracking results on these two dynamic NRTs. Note that four textons are specified to initialize tracking in Figure 3.17 as each of these textons has different appearance. The 
appearance of textons vary rapidly in these two videos because of surface refraction and motion blur. Despite these difficulties, our algorithm is able to track these highly dynamic and varied textons successfully. These two experiments demonstrate that, even without occlusion, dynamic NRT tracking poses challenging issues to the robust optical flow algorithm and the Lucas-Kanade algorithm.

The textons of the underwater texture are modeled as a loosely coupled MRF allowing flexible motion of textons. Figure 3.18 shows an example of tracking loosely coupled textons. In this example, a texton is defined as a local patch around the head region of a person. The marching motion presents a relatively large global motion and small local deformation of individual textons compared to the motion of tightly coupled textons. Also, the appearance of textons varies more due to shadows. The robust optical flow tracking results and the Lucas-Kanade results are shown in Figure 3.18(a)(b). The underwater texture and crowd marching examples show that our algorithm is able to handle large illumination changes, rapid geometric deformation, and intensity variations in the tracking process.

Tracking Dynamic NRTs with Occlusion. Occlusion is one of the major challenges in dynamic texture tracking. Textons may leave/enter the scene, or be occluded by other objects or other textons on a folded surface. We tested our tracking algorithm on different cloth motion under different degrees of occlusions.

Figure 3.19 is a comparison of our tracking results with Guskov's [2002]. Guskov proposed a tracking algorithm that is specially designed for tracking black-white square patterns (section 3.1.2). This example shows that our tracking algorithm achieves similar performance with Guskov's algorithm. While our algorithm can handle general NRT, Guskov's algorithm is specially designed for real-time tracking of black-white checkerboard patterns. We also compared tracking results with a result by manually tracking (ground truth). The root mean square error of Guskov's result and ours against hand-labeled ground truth are 2.94 and 2.57 pixels respectively.

Figure 3.20(a) shows our tracking result on a fabric pattern under self-occlusion and textons leaving/entering the scene during tracking. The lattice, visible textons, and occluded textons are shown in red, yellow, and cyan colors respectively. Figure 3.20(b) shows the visibility map of textons where blackened regions correspond to detected occluded textons and all visible textons are geometrically aligned. 

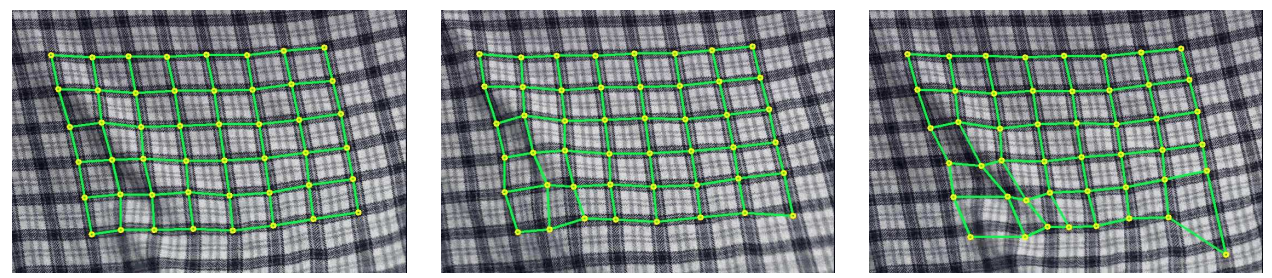

(a) Robust optical flow tracking results at frame 1, 25, and 86 (slow.avi)
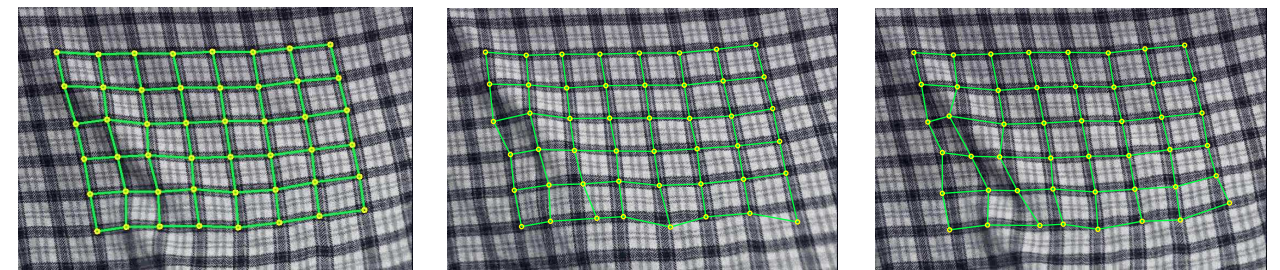

(b) Lucas-Kanade tracking results using affine transformation parameters
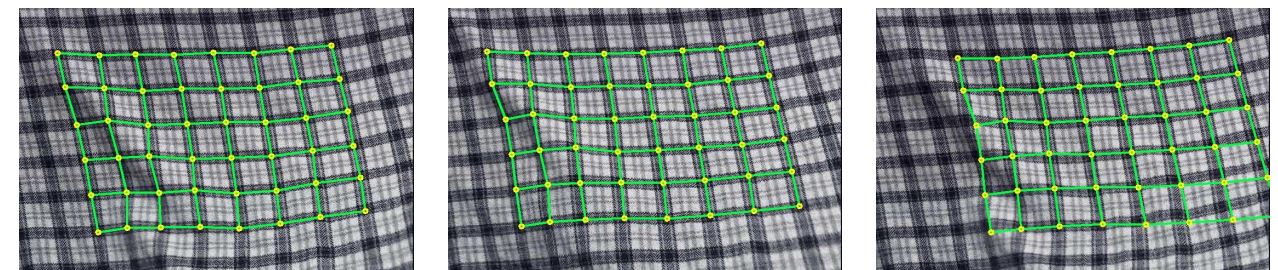

(c) Active appearance model tracking results
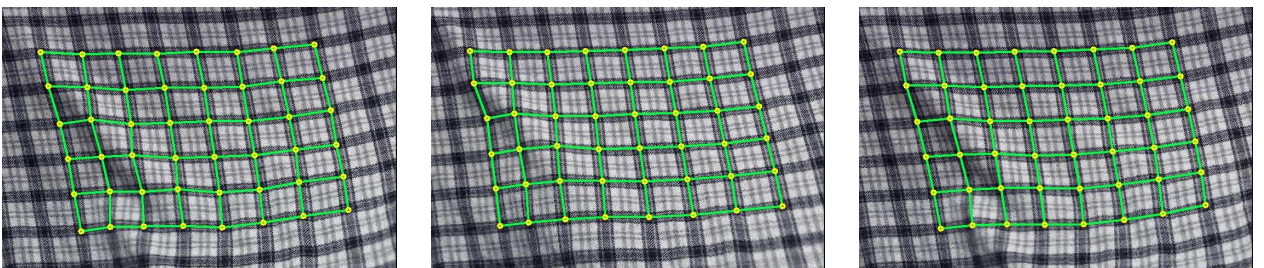

(d) Our tracking results (slow.avi)

Figure 3.15. Lattice tracking results of a dynamic near-regular texture on waving cloth. One can observe that the tracking error accumulates quickly in the optical flow results.

Figure 3.21 shows another tracking result where a fabric pattern is being folded. There are a few textons totally occluded in the middle and two occluded by a finger in the bottom-right region (Figure 3.21(b)). Also, there are new textons added to the lattice during the tracking process (compare the bottom right corner of two visibility maps in Figure 3.21(a) and (b)). When the texton is at the boundary of a lattice, the BP inference result for the texton is less reliable since it receives messages from fewer neighboring nodes. This is the reason why there are some tracking errors in the cyan lattice at boundary, e.g, top-middle in Figure 3.21(c). Although our algorithm can successfully track textons through occlusion, there is another interesting research problem: 

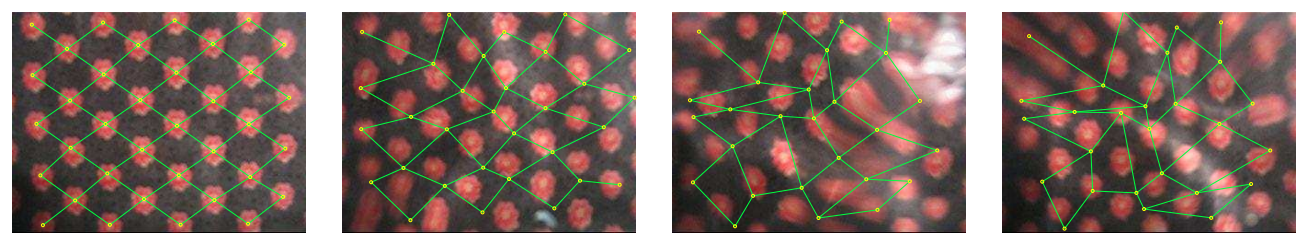

(a) Robust optical flow tracking results
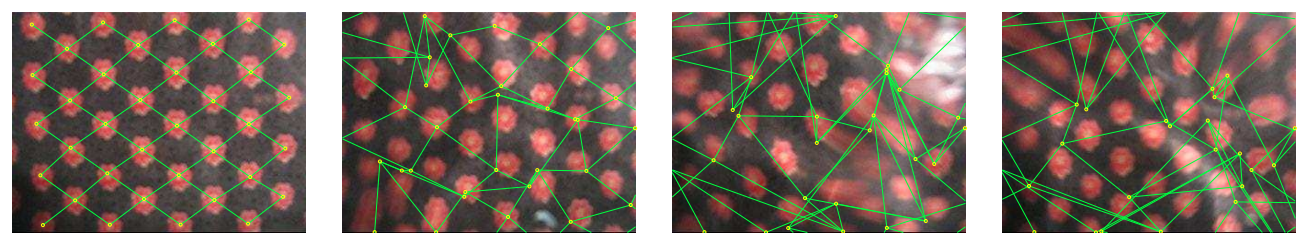

(b) Lucas-Kanade tracking results using affine transformation parameters
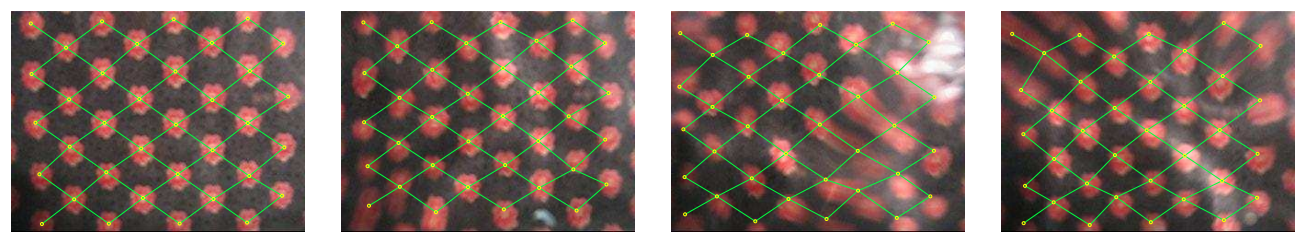

(c) Our tracking results

Figure 3.16. Tracking results of an underwater texture at frame 1, 50, 91, and 100 (water_tracked.mov). Note that there are serious motion blurs and large lighting variations due to highlights in the video. Texture replacement results can be seen in water_replaced.mov.

can we infer the positions of textons when they are occluded. One way to solve this problem is to modify the MRF model such that it can represent a folded topology under occlusion. We would like to explore this problem in the future.

\subsubsection{Validation and Comparison}

In this section, we conduct several experiments to validate our MRF model and compare our tracking algorithm with mesh-based tracking and multi-target tracking algorithms. We first verify if the 12-neighbor configuration is the best setting for dynamic NRTs by testing our tracking algorithm with 8- and 16-neighbor configuration on underwater texture motion. We test our tracking algorithm with multiple texton templates and single texton template settings and texton detection algorithm initialized at different positions. Finally, we compare the performance of our tracking algorithm with deformable object tracking and multi-target tracking algorithms.

Validation of 12-neighbor configuration. The 12-neighbor configuration in our MRF model is adopted from the spring configuration in physics-based cloth simulation. 

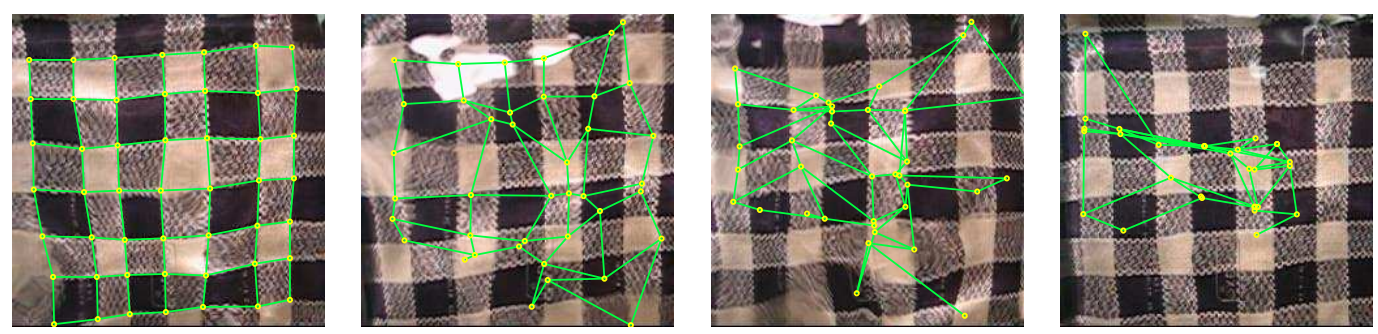

(a) Robust optical flow tracking results
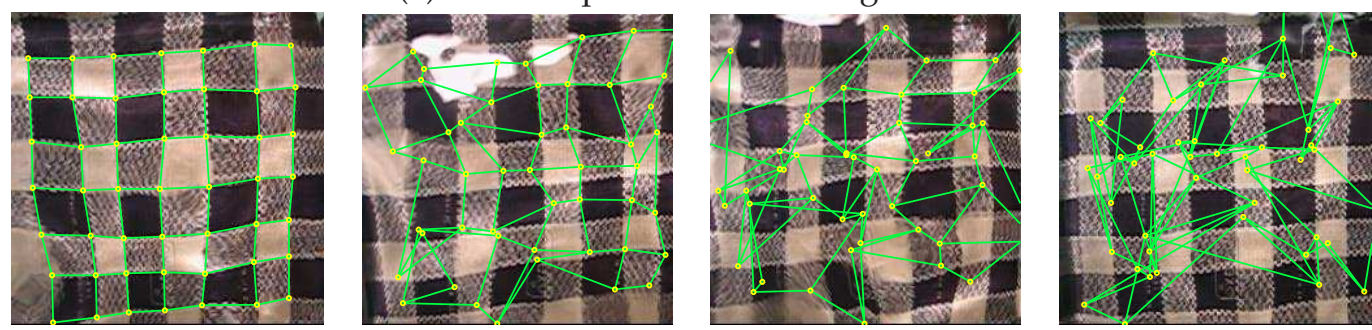

(b) Lucas-Kanade tracking results using affine transformation parameters
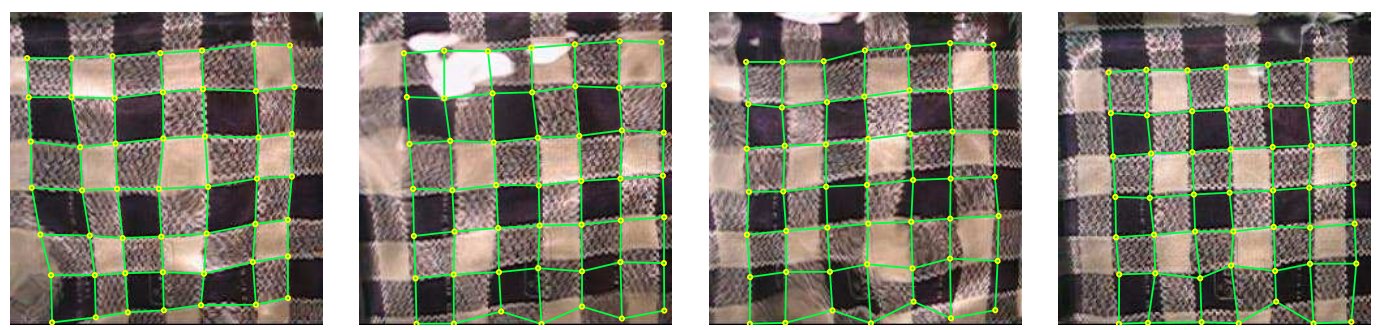

(c) Our tracking results

Figure 3.17. Tracking result of another underwater texture at frame 1, 70, 100 and 200. There are serious motion blurs and large lighting variations due to highlights in the video.

Our tracking results show that this 12-neighbor configuration works well for different types of dynamic NRTs, from highly dynamic underwater textures to slowly varying fabric textures. To further validate that the 12-neighbor MRF model is appropriate for dynamic NRTs, we tested our tracking algorithm with different neighborhood configurations. Figure 3.22 shows several static frames of tracking results with different number of neighbors used in the MRF model. From this experiment, we found that an 8-neighbor configuration cannot provide sufficient constraints to maintain the lattice structure while a 12-neighbor configuration introduces too strong structural constraints. This makes the tracking algorithm less adaptive to highly dynamic motion. Once the algorithm loses tracking a texton, the algorithm cannot catch the texton again (Figure 3.22). 

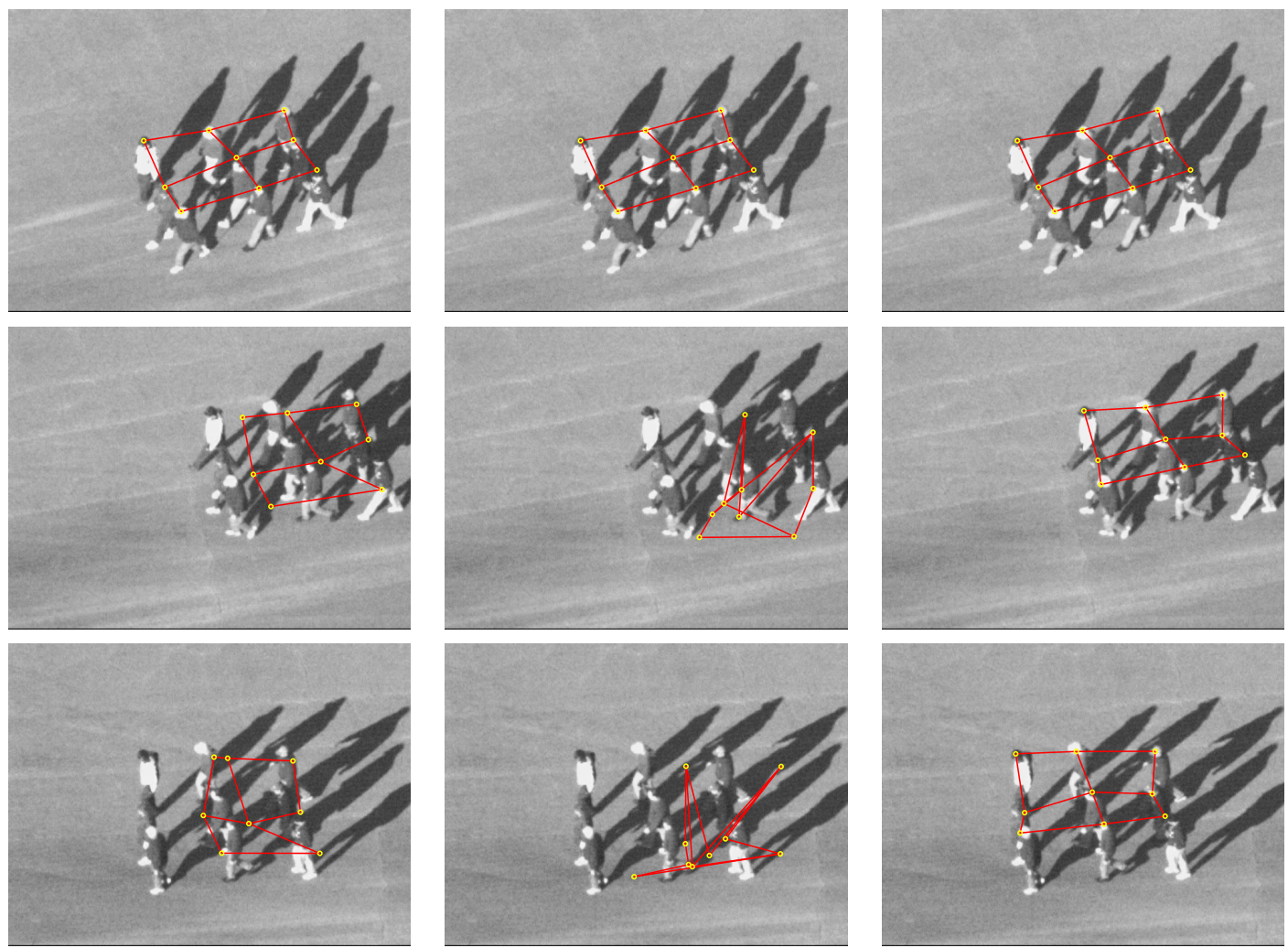

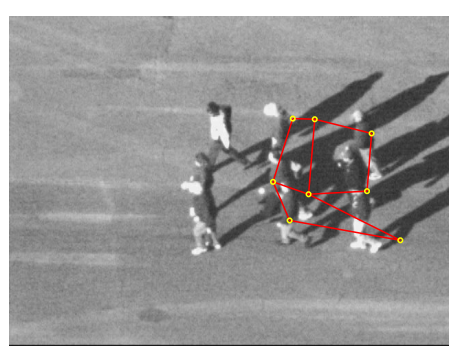

(a)

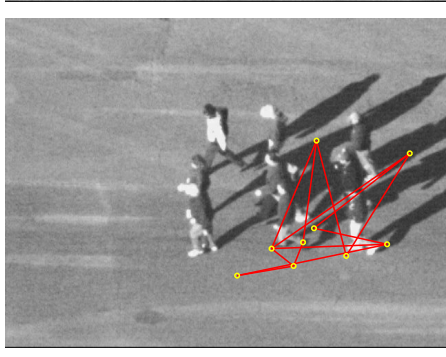

(b)

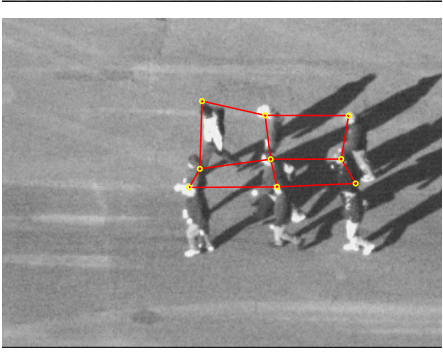

(c)

Figure 3.18. Tracking results at frame 10,110, 210, and 310 (crowd.avi). (a) Robust optical flow tracking results. (b) Lucas-Kanade tracking results using affine transformation parameters. (c) Our tracking results.

Multiple Texton Templates vs. Single Texton Template. We explored if PCA can be used to represent multiple texton templates. This would allow a more compact representation of texton templates. Figure 3.23 plots the number of PCA bases ( $95 \%$ energy) used during tracking (see Figure 3.24 for tracking results). At the first frame, only one basis is needed since a single texton is used. As the tracking proceeds, the number of PCA bases increases and reaches its maximum of 34 at frame 127. If no PCA is applied, 70 texton templates are used during tracking process. Figure 3.24 compares the tracking results of multiple and single texton templates at several frames. The tracking 


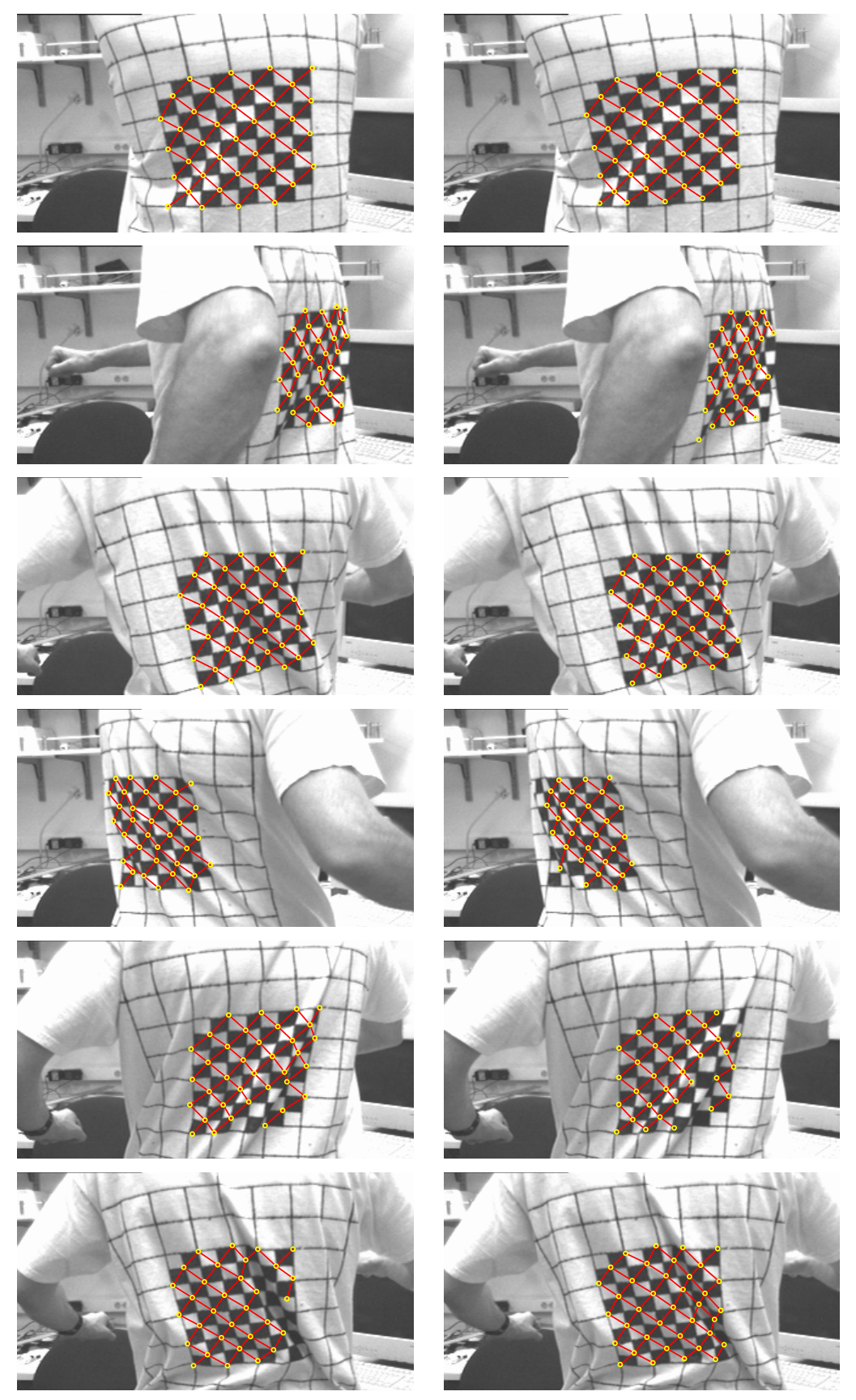

Figure 3.19. Left column: tracking results of [Guskov, 2002] (Courtesy of Igor Guskov). Right column: our tracking results. This example shows that our tracking algorithm achieves similar performance with Guskov's algorithm that is specially designed for black-white-square patterns. The root mean square error of Guskov's results and ours against hand-labeled ground truth are 2.94 and 2.57 pixels respectively. 

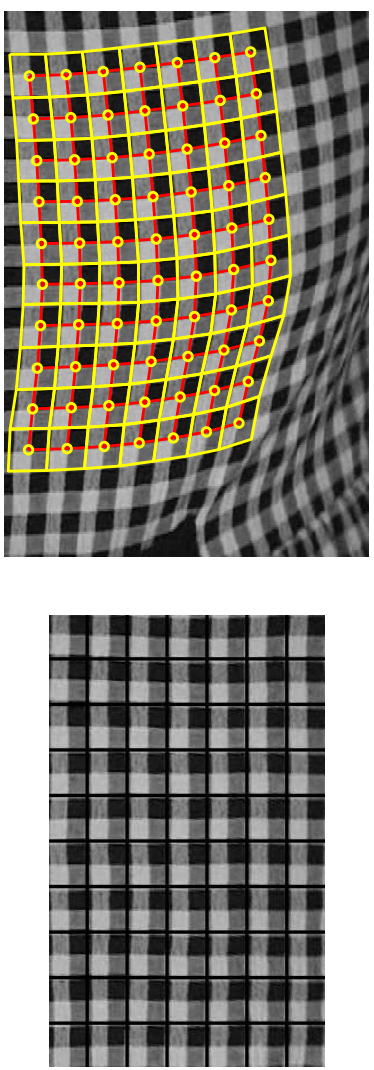

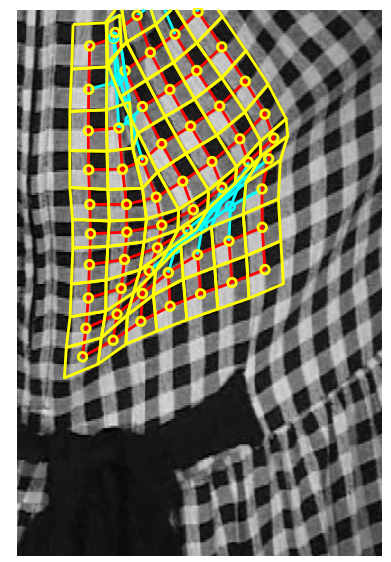

(a)

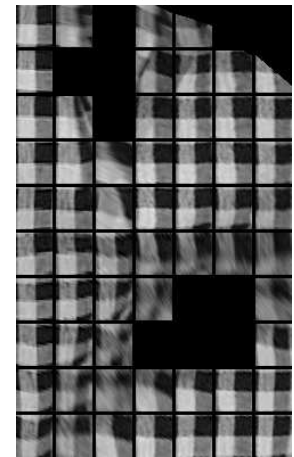

(b)
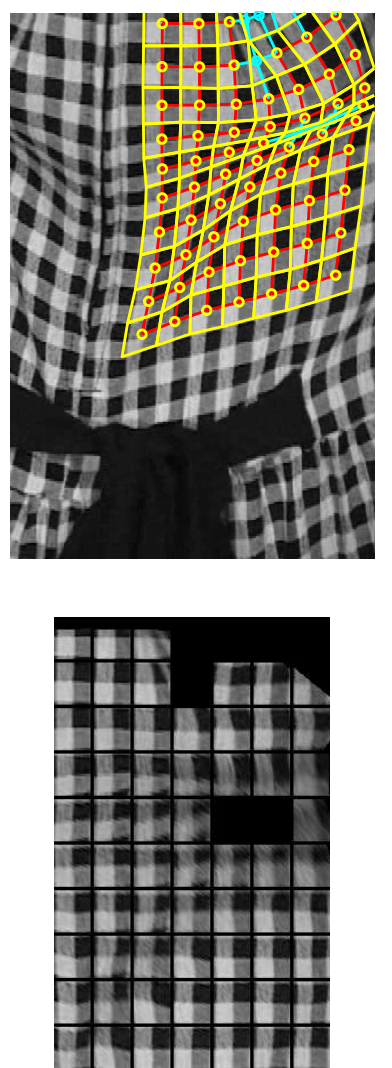

Figure 3.20. Tracking results of a fabric pattern under occlusion (dress.avi). The visible lattice, occluded lattice, visible textons, and occluded textons are shown in red, cyan, yellow, and cyan color. The visibility map shows visible aligned textons.
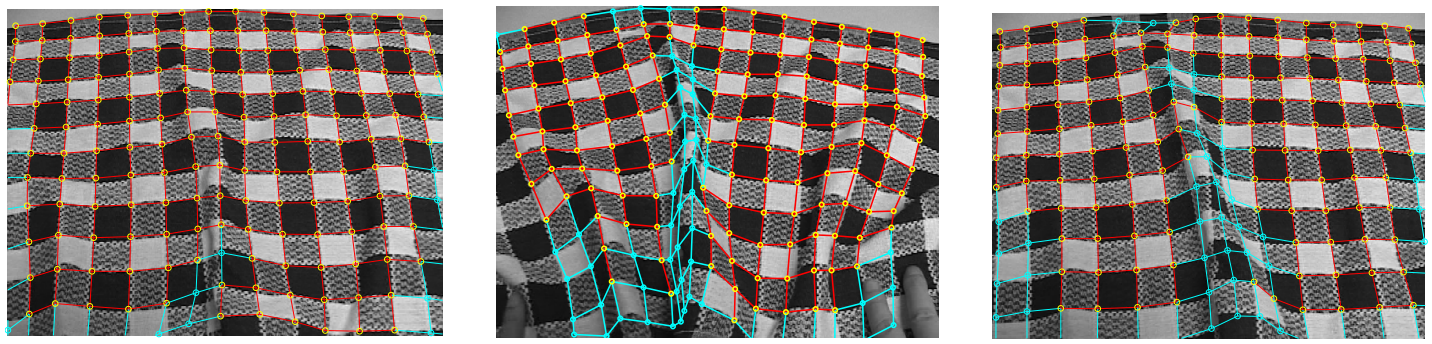

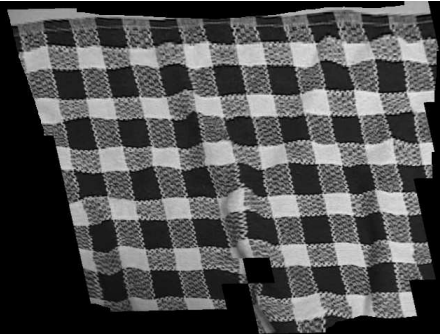

(a) frame 25

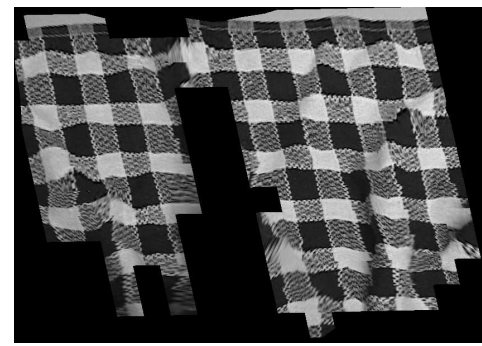

(b) frame 50

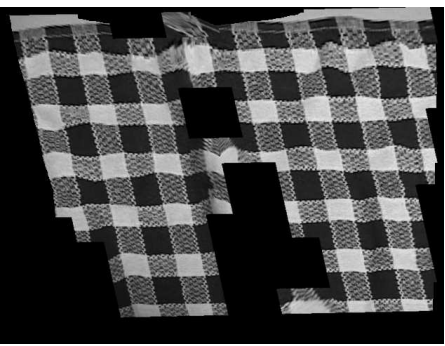

(c) frame 100

Figure 3.21. Top row: tracking results of a folding fabric pattern (towel.mov). There are a few textons totally occluded in the middle and two textons are occluded by a finger in the lower-right region. Bottom row: visibility map 

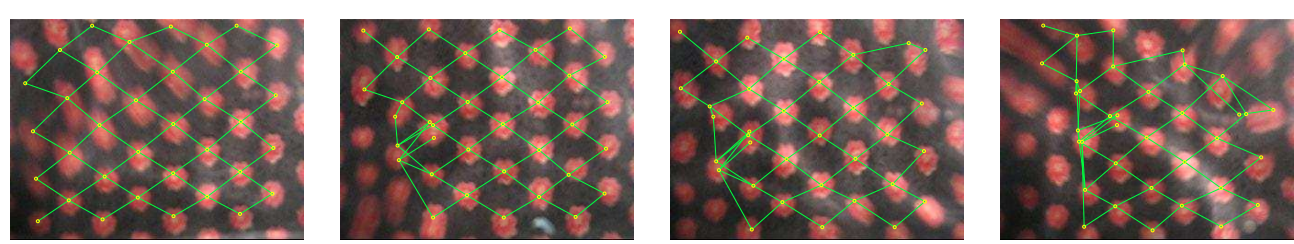

(a) Tracking results with the 8-neighbor configuration
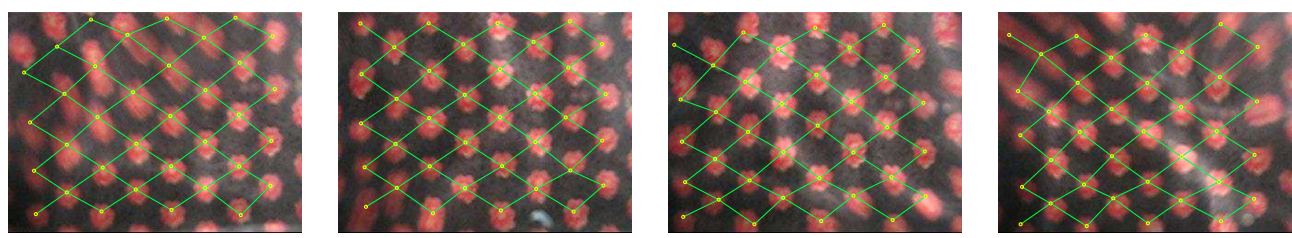

(b) Tracking results with the 12-neighbor configuration
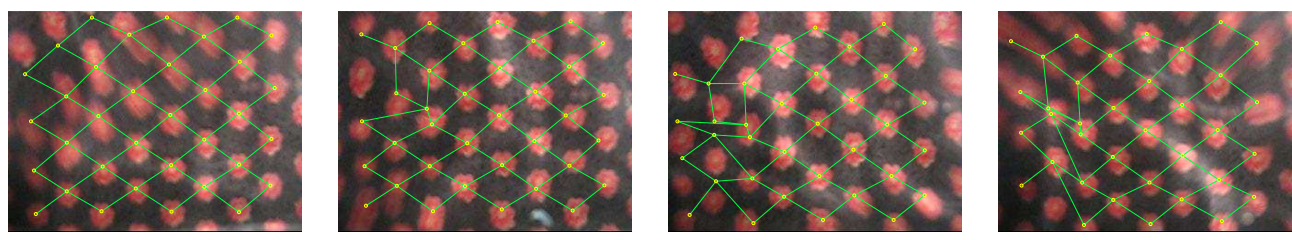

(c) Tracking results with the 16-neighbor configuration

Figure 3.22. Tracking results of an underwater texture at frame 25, 50, 75, and 100 (numNbrsExp.avi).

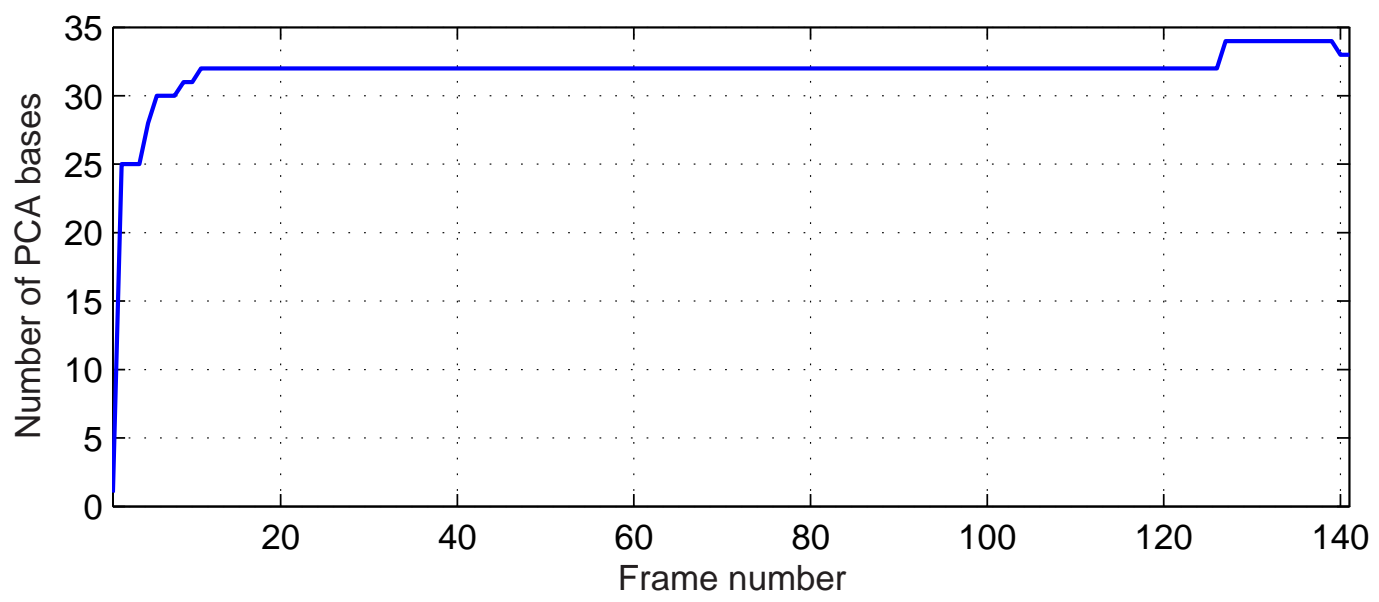

Figure 3.23. Plot of number of PCA bases (95\% energy) used to represent texton templates in the tracking process. At the first frame, only one basis is used since a single texton template is used. As tracking procee

results show that the PCA texton template model, although providing more compact representation, has slightly larger tracking error than using multiple texton templates. 

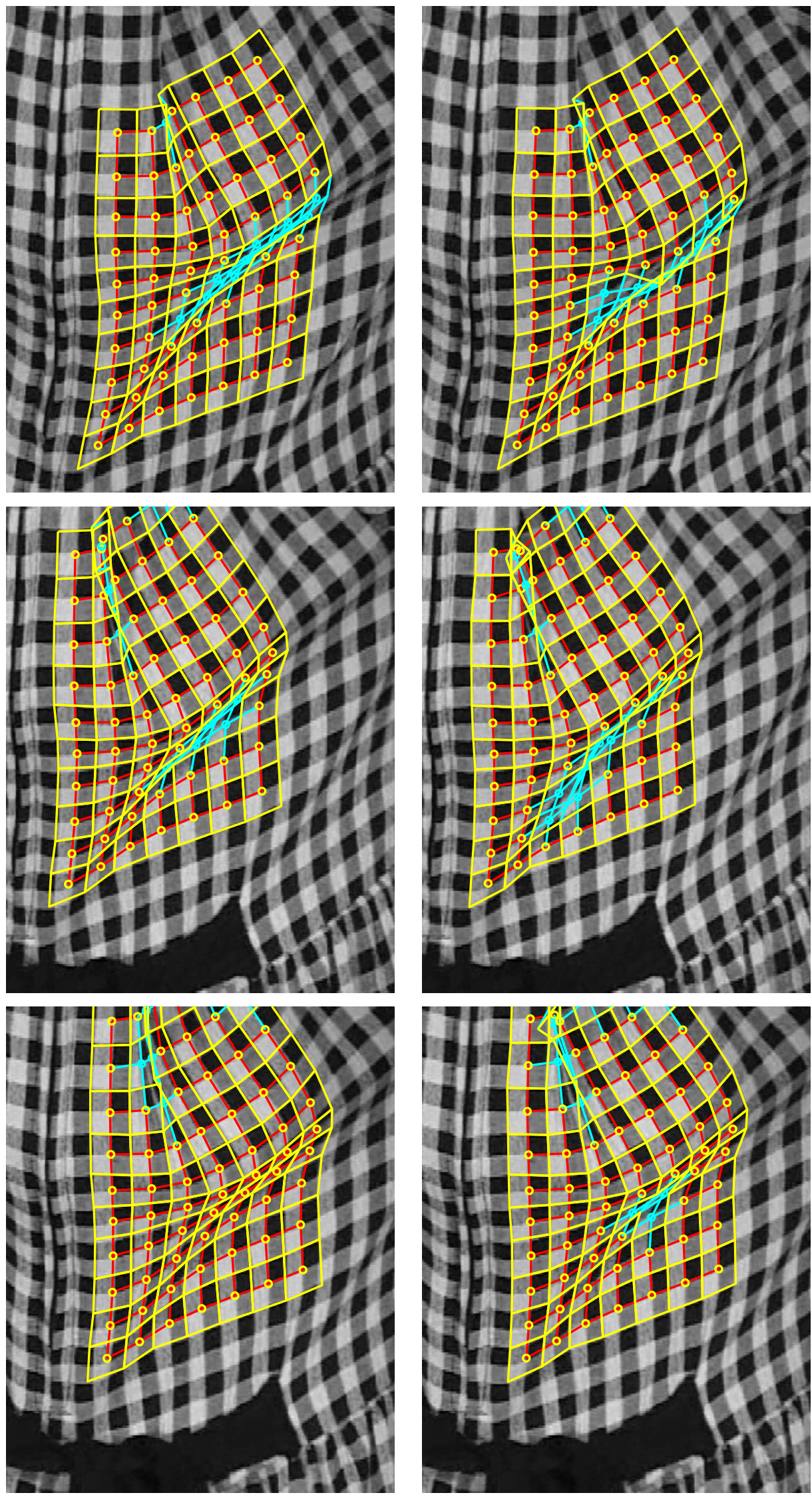

Figure 3.24. Comparison of tracking results using multiple texton templates (left column) and single texton template (right column). One can observe that the tracking results of multiple texton templates are more accurate. 
Comparison of Texton Detection with Different Initial Positions. The purpose of this comparison is to see how the position of the initial texton (the first texton specified by the user) affects texton detection result. We start the texton detection algorithm (section 3.5.1) at different initial positions. Figure 3.25 and 3.26 show the texton detection results of fabric textures on a towel and a dress. In Figure 3.25(d), two textons at the top-left corner are not detected successfully because the image intensities of these textons are much darker than those of the initial texton. Also, there are minor detection errors at the bottom-left corner. These errors are mainly attributed to the perspective distortion and nonlinear deformation in the middle part of the towel. Similar to temporal tracking, the spatial tracking error accumulates when the lattice grows from the bottom-right corner to the bottom-left corner. The texton detection results in Figure 3.26 do not differ significantly despite different initial positions. The only texton detection error appears in Figure 3.26(d) where the textons in the top-middle row are not precisely detected.

In general, both examples in Figures 3.25 and 3.26 show that the spatial detection algorithm is not very sensitive to different initial positions although the spatial detection algorithm may work better on NRTs that have denser texton densities and are on surfaces with smooth deformations (comparing NRTs in Figures 3.25 and 3.26).

Comparison with Deformable Object Tracking and Multi-target Tracking. Dynamic NRTs exhibit wide range of motion characteristics, from slowly periodic motion to rapidly moving motion, and from surface deformation to loosely coupled crowd motion. If we arrange different dynamic NRTs based on structural constraints among individual textons, these dynamic NRTs form a spectrum. It appears that on one end of the spectrum, dynamic NRTs may be considered as a deformable object tracking problem while on the other end, dynamic NRTs can be treated as a multi-target tracking problem. It would be interesting to compare the performance of our tracking algorithm against deformable tracking and multi-target tracking algorithms.

Among deformable object tracking algorithms, we chose Active Appearance Model (AAM) [Cootes et al., 1998] for comparison since most deformable object tracking algorithms are developed based on AAM or similar concept [Matthews and Baker, 2004]. These algorithms use a mesh to represent the shape of a deformable object and apply an image alignment algorithm to fit the appearance of an input image sequence with 


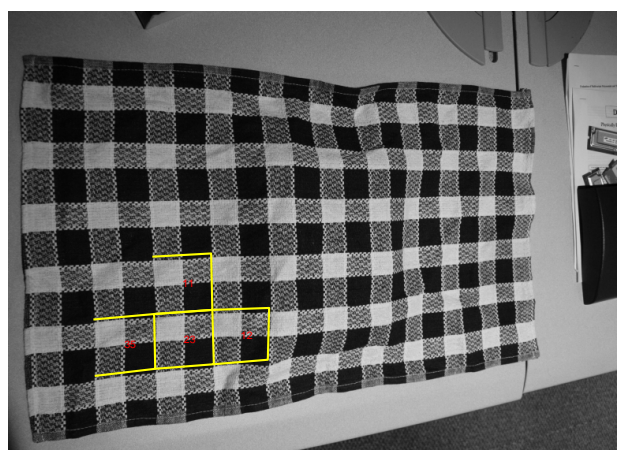

(a)

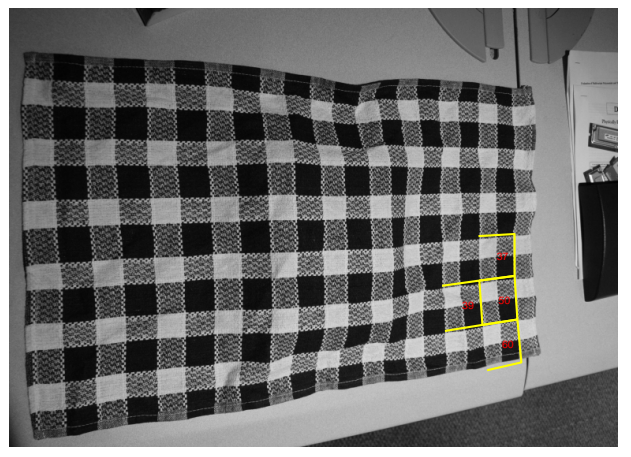

(c)

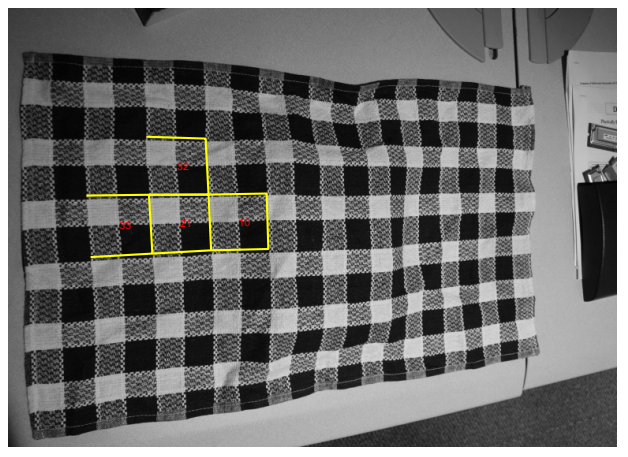

(e)

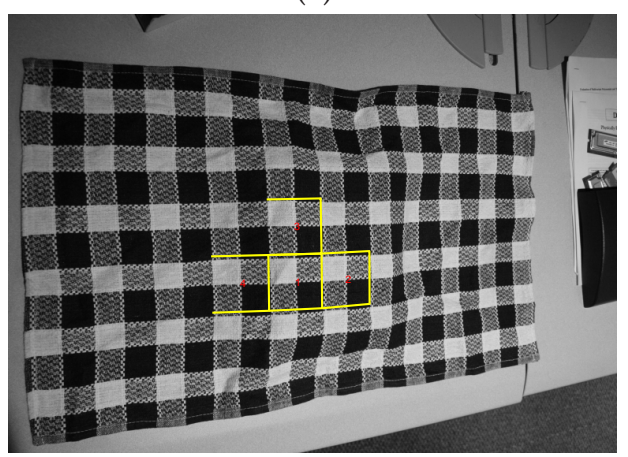

(g)

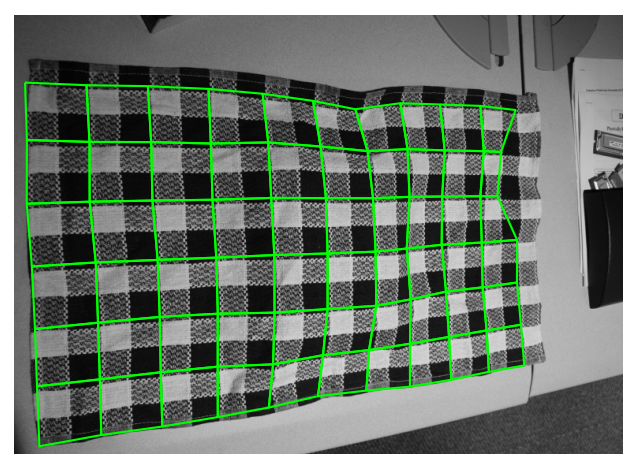

(b)

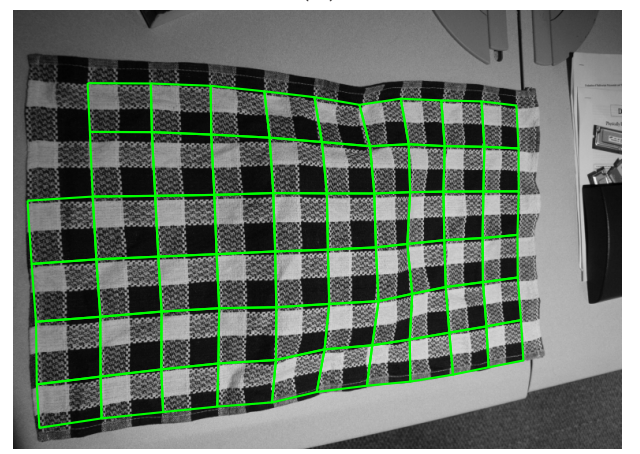

(d)

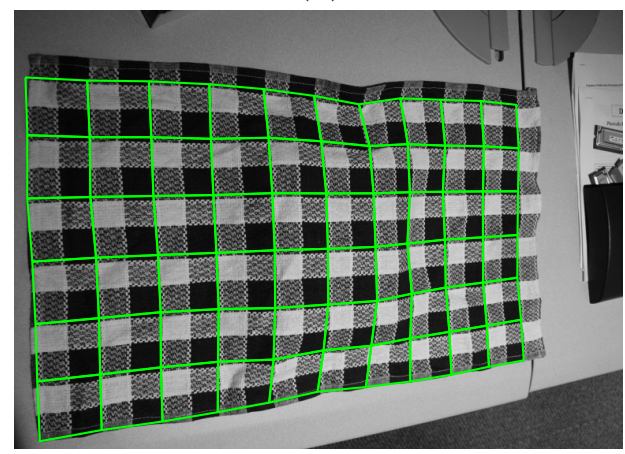

(f)

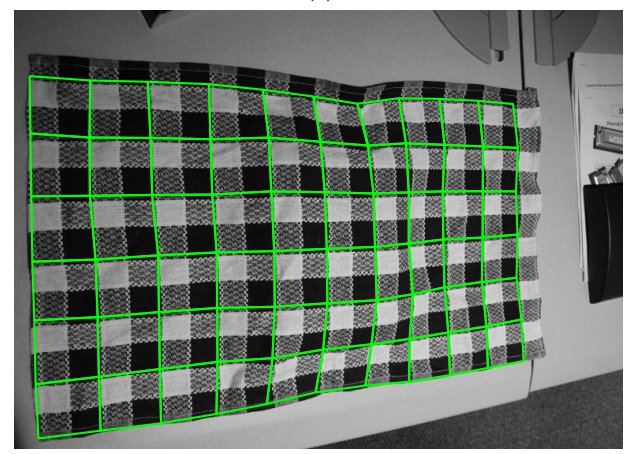

(h)

Figure 3.25. Texton detection results starting at different initial positions. $(\mathrm{a})(\mathrm{c})(\mathrm{e})(\mathrm{g})$ are initial textons and $(\mathrm{b})(\mathrm{d})(\mathrm{f})(\mathrm{h})$ are texton detection results correspondingly. Two textons at the top-left corner in (d) are not detected successfully because the image intensities of these textons are much darker than those of the initial texton. 


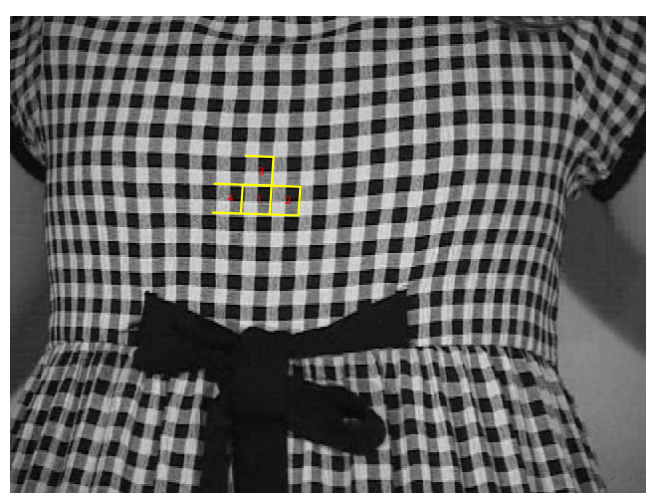

(a)

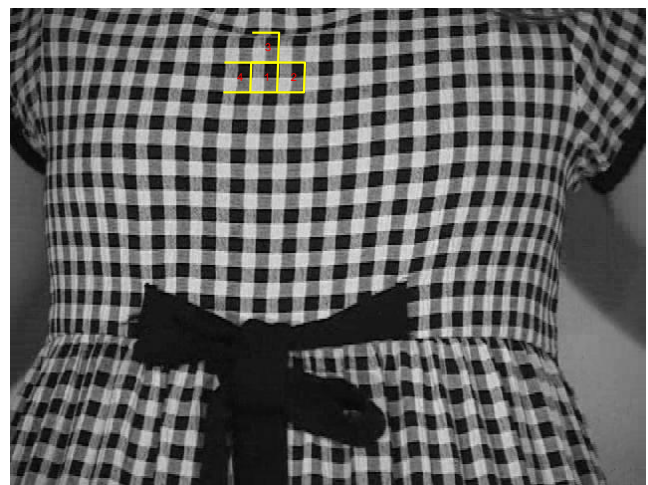

(c)

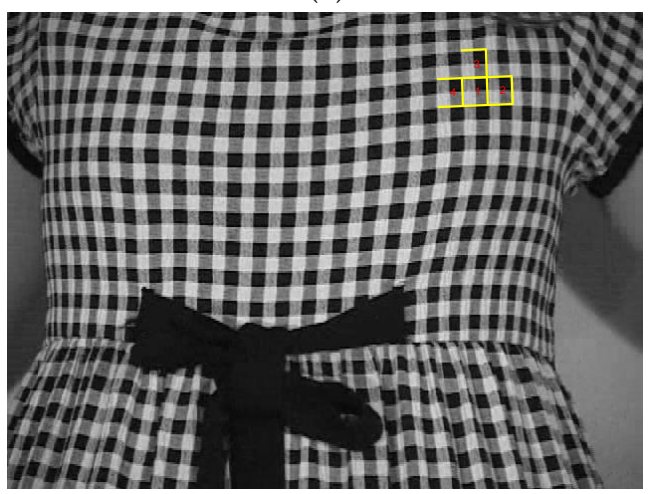

(e)

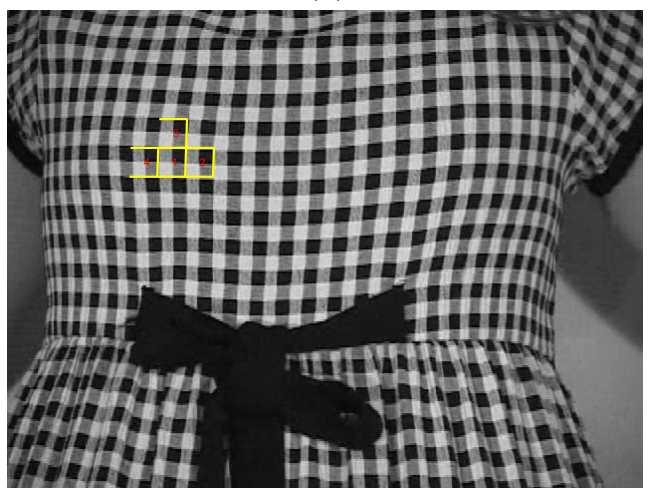

(g)

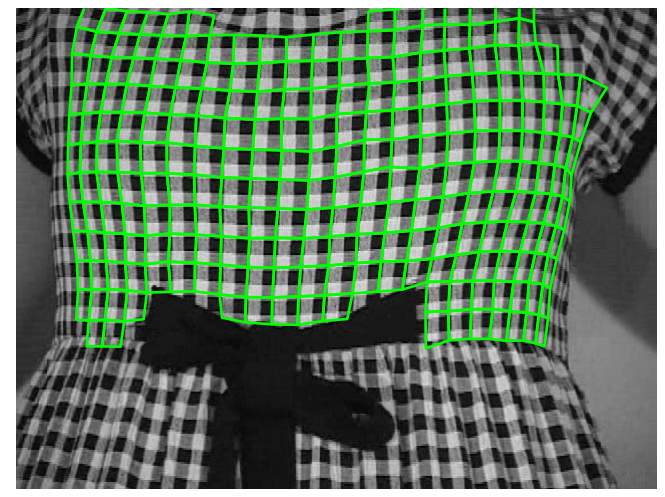

(b)

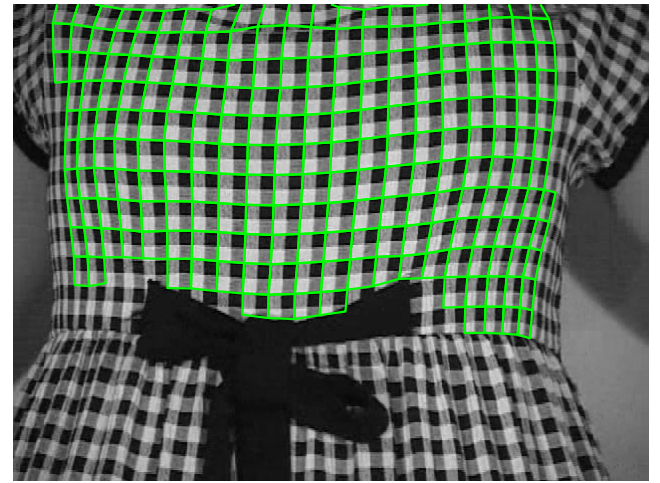

(d)

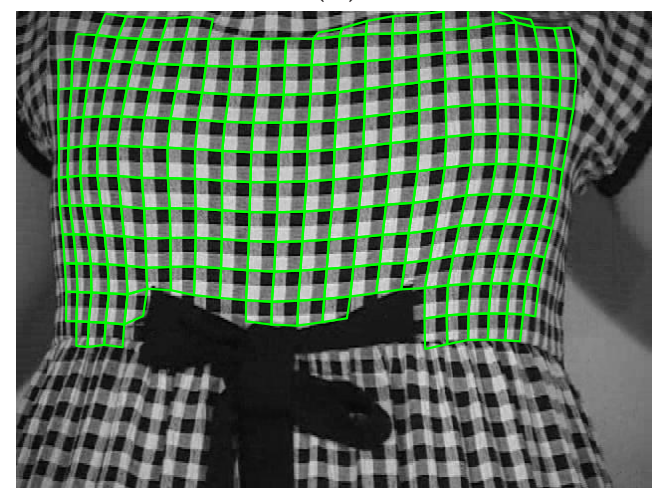

(f)

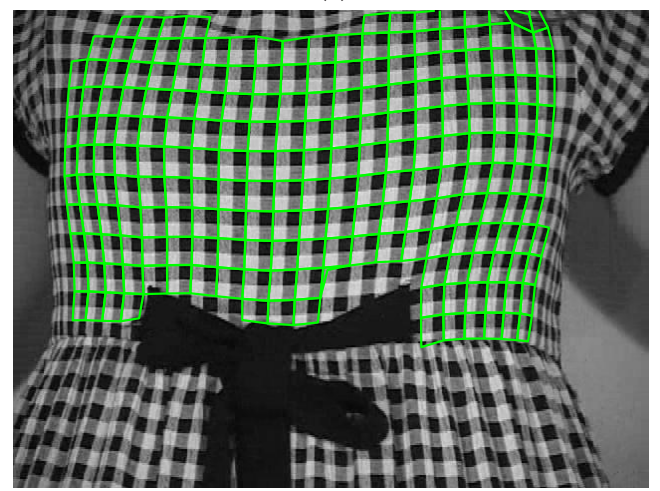

(h)

Figure 3.26. Texton detection results starting at different initial positions. (a) (c)(e)(g) are initial textons and $(b)(d)(f)(h)$ are texton detection results correspondingly. 
a modal image or an appearance model constructed by a set of training images. Figure 3.15(c) shows the AAM tracking result of a slowly varying dynamic NRT using the AAM code implemented by Matthews and Baker [2004]. In this experiment, we selected 10 frames (uniformly sampled) from the input image sequence to construct an AAM model. The 2D meshes used for constructing the AAM model were manually labeled. The lattice drifts due to ambiguous correspondences although the lattice structure is maintained in the AAM tracking result. Figure 3.27 shows image frames where drifting occurs in the AAM tracking result.

The reason that AAM-based approaches cannot track dynamic NRTs can be further analyzed by comparing the differences between AAM and our tracking algorithm. There are two important differences. First, our MRF model integrates a spring-networklike statistical lattice structure model and a registration-based image observation model. The role of image observation model is similar to image alignment algorithm in AAMbased approaches; however, the additional lattice structure model makes a crucial difference. It allows local nonlinear deformation while AAM only models global linear deformation. If a surface deformation cannot be represented by an AAM, AAM may lose tracking of textons and the whole lattice is attracted by neighboring textons with similar appearance (Figure 3.27). Second, we use a linear dynamic model (Equation (3.20)) to predict the position of lattice in the next frame. The predicted lattice position provides a better initial condition for the image alignment algorithm such that the image alignment process is more likely to converge to the correct solution.

We also compared the performance of our tracking algorithm against multi-target tracking algorithms. In particular, we chose $\mathrm{Yu}$ and $\mathrm{Wu}$ 's tracking algorithm [ $\mathrm{Yu}$ and $\mathrm{Wu}, 2005]$ as they also use an MRF to represent the spatial constraints between targets. Specifically, each target is tracked by a tracker, and these trackers collaborate with each other under a MRF model to resolve ambiguous correspondences among multiple identical targets.

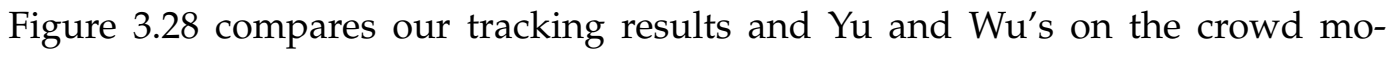
tion. This comparison shows that our tracking algorithm can keep tracking all targets through the entire sequence while $\mathrm{Yu}$ and $\mathrm{Wu}$ 's algorithm may lose tracking of targets at short periods. To quantitatively compare their results and ours, we manually track the lattice every 5 frames in the video. The total root mean square errors (RMSE) of Yu 


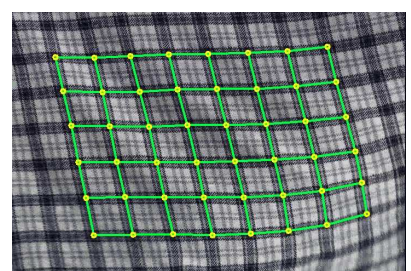

frame 41

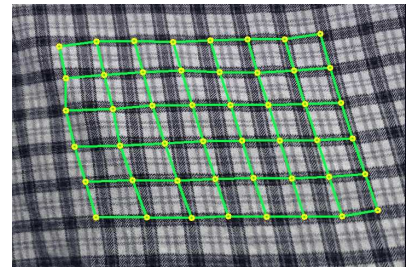

frame 50

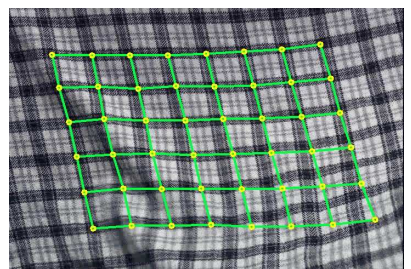

frame 44

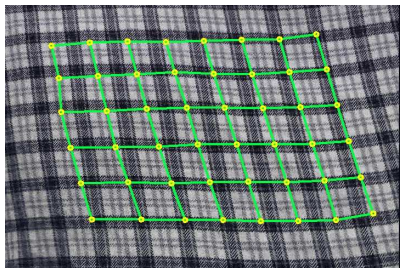

frame 53

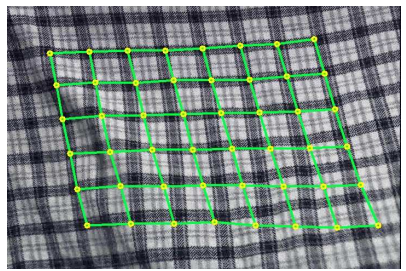

frame 47

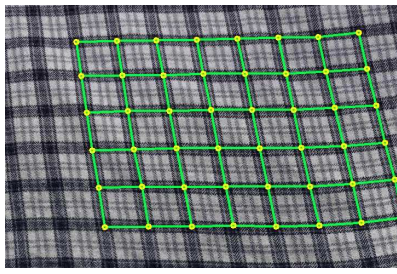

frame 56

Figure 3.27. A short image sequence showing the drifting of lattice in the AAM tracking result. The lattice drifts because the AAM is not able to local nonlinear deformation and tracking can not be recovered once the lattice drifts to other texton locations.

and Wu's results and ours are 25.9 and 20.2 pixels respectively. Figure 3.29 is an RMSE plot of tracking results.

The comparison with deformable object tracking and multi-target tracking algorithms demonstrate the effectiveness of our lattice-based MRF model on dynamic NRT tracking. The comparison also shows that our tracking algorithm not only provides a unified framework for tracking dynamic NRT under a wide range of motion but also outperforms algorithms that are specialized at certain type of motion-deformable objects and multi-targets.

\subsection{Dynamic NRT Manipulation}

An immediate benefit of tracking textons is that a more precise appearance model of a dynamic texture can be obtained. The appearance model combined with the geometric model can be used to generate dynamic textures in different manners other than synthesis. Specifically, dynamic NRT manipulation can be achieved by manipulating the sequence of geometric and lighting deformation fields of a dynamic texture. Three types of manipulations are addressed in this thesis: texture replacement, superimposing, and video synthesis. Maintaining temporal coherence is an important issue in synthesizing and manipulating dynamic textures. It is especially a challenge for handling dynamic NRTs because the textons of an NRT provide strong cues for human eyes to 

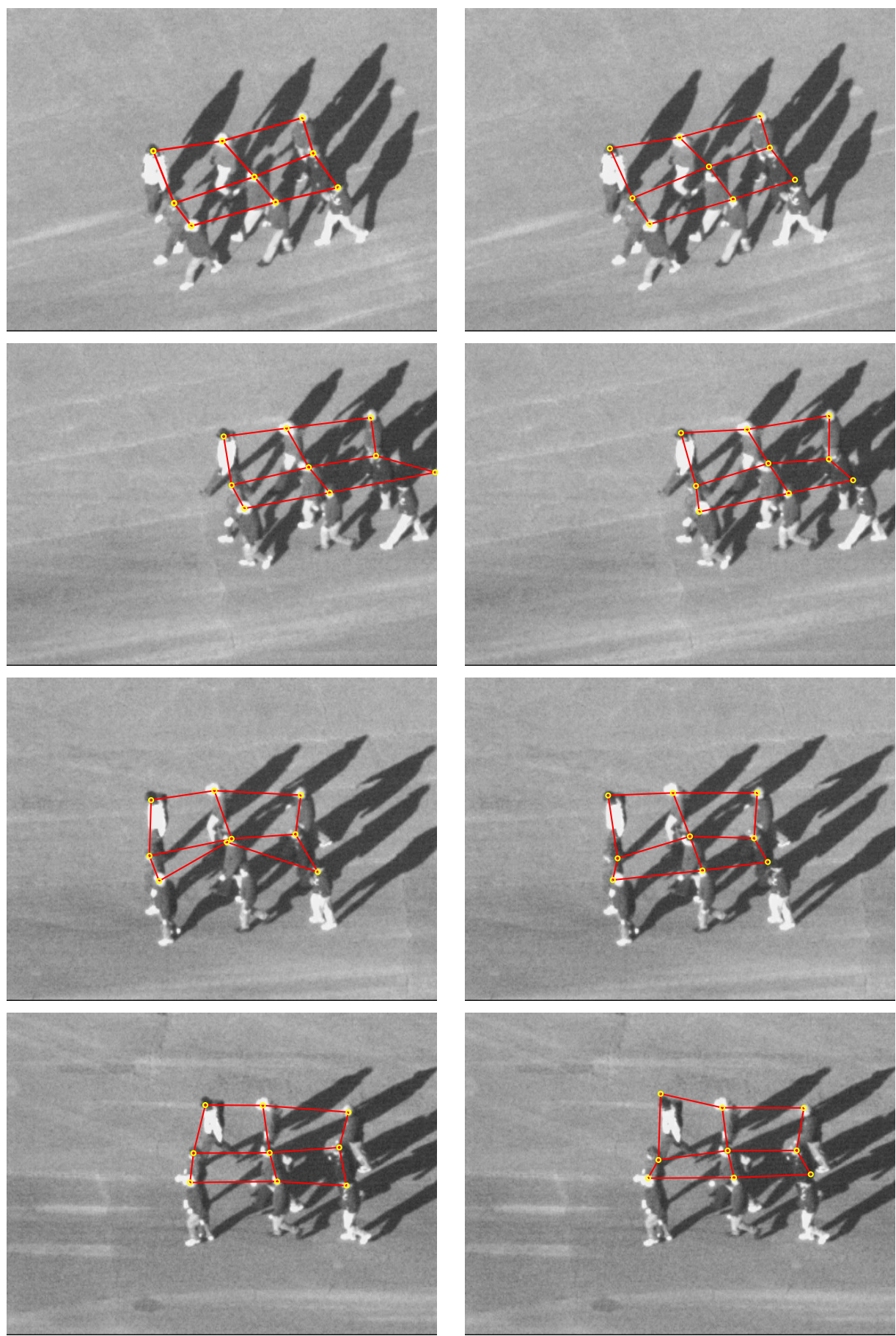

(a) Yu and Wu's tracking result [2005]

(b) Our tracking result

Figure 3.28. Comparison of multi-target tracking result (courtesy of Ting Yu and Ying $\mathrm{Wu}$ [2005]) and our tracking result at frame 10, 110, 210, and 310. Our tracking algorithm is able to keep tracking all targets through the entire sequence while $\mathrm{Yu}$ and $\mathrm{Wu}$ 's algorithm may lose tracking of targets at short periods. 


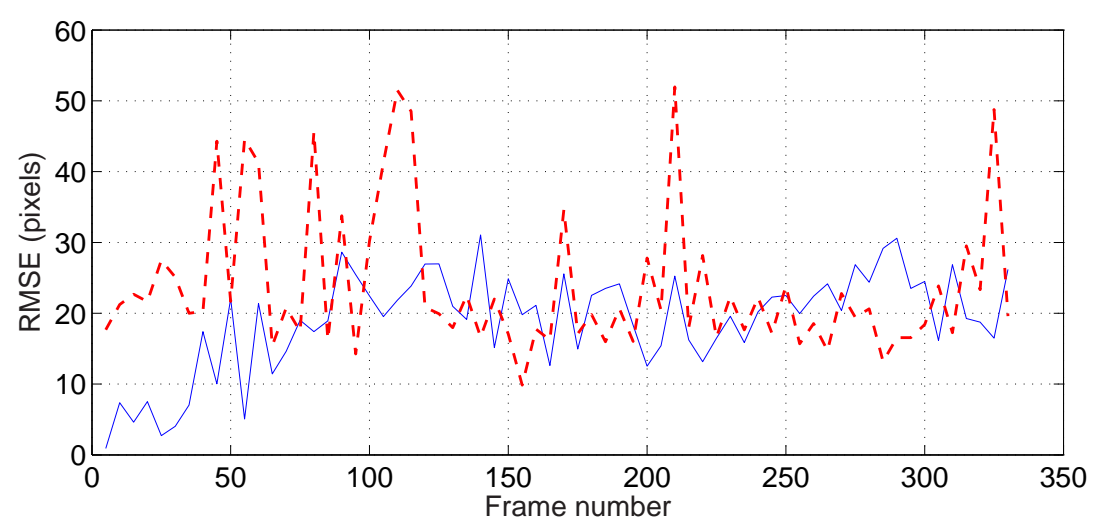

Figure 3.29. Comparison of root mean square error against the hand-labeled ground truth. The red dash line and blue line corresponds to the RMSE curve of $\mathrm{Yu}$ and $\mathrm{Wu}$ 's and our tracking result. The total RMSE of $\mathrm{Yu}$ and Wu's results and ours are 25.9 and 20.2 pixels respectively.

associate temporal correspondences, even a small discontinuity in the motion of textons would be noticeable.

\subsubsection{Dynamic NRT replacement}

Aiming to achieve photorealistic and appealing texture replacement, we develop an effective framework to preserve the geometric and lighting effects while maintaining the temporal coherence in the replacement results. Temporal coherence is addressed by smoothing the geometric and lighting DFs spatiotemporally. The purpose of smoothing is analogous to one the functions of human vision system in which the velocity information is integrated spatiotemporally to obtain unambiguous and coherent perception of motion [McKee and Welch, 1985; Watamaniuk and Sekuler, 1992]. The smoothed geometric and lighting DFs can then be applied to any texture to achieve realistic and coherent video texture replacement. When there is more than one NRT patch in an image frame, spatiotemporal stitching is applied to combine replacement results from different patches. Figure 3.30 shows an overview of dynamic NRT manipulation.

Geometric and Lighting Deformation Field Extraction. The appearance variations of a dynamic near-regular texture is modeled as a regular texture under the effect of a sequence of geometric DFs and lighting DFs. An important step of the deformation field extraction algorithm (section 2.2) is to identify the lattice structure of the nearregular texture. Once the lattice of a dynamic NRT is obtained, a sequence of DFs can 


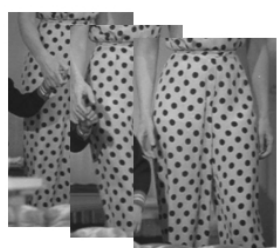

Input video

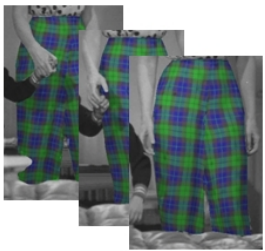

Output video
Lattice Tracking

Spatiotemporal Stitching

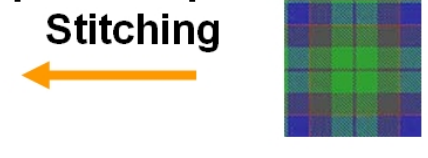

New texture
Deformation

Field

Extraction

Geometric DF's

Deformation

Field

Smoothing

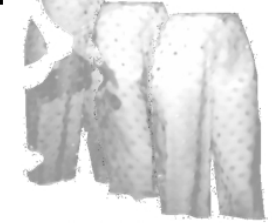

Lighting DF's

Figure 3.30. Overview of dynamic NRT replacement.

be computed accordingly. Figure 3.35(b) and 3.33(c) show examples of extracted lighting deformation fields.

When dealing with dynamic NRTs in videos, the geometric and lighting deformation fields are first computed frame by frame. Since the texture in each frame is warped to the same geometrically-regular state (same lattice), geometric deformation fields can be considered as a texture mapping function that directly maps pixels from texture coordinate to image space coordinate. When an NRT's lattice has occluded nodes, these nodes are not used in geometric deformation field computation as it does not need to map pixels to occluded regions.

Geometric Deformation Field Smoothing. The trajectories of lattice points from tracking is usually noisy and the geometric deformation field computed from noisy trajectories would not be temporally smooth. If this geometric deformation field is directly applied to a texture, the texture would pop around in the generated video. To maintain the temporal coherence, we smooth the lattice motion through an optimization process. The goal of the optimization is to find a smooth trajectory $p_{i}$ with minimum deviation from $q_{i}$ where $q_{i}(t) \in \mathbb{R}^{2}$ is the initially tracked position of lattice point $i$ at frame $t$ and $p_{i}(t) \in \mathbb{R}^{2}$ is the optimization variables. The energy function, $E_{i}=E_{d}+\beta \cdot E_{s}$, is defined 
as follows,

$$
\begin{aligned}
E_{d} & =\sum_{t=1}^{T}\left\|p_{i}(t)-q_{i}(t)\right\|^{2} \\
E_{s} & =\sum_{t=1}^{T-1}\left\|p_{i}(t)-p_{i}(t+1)\right\|^{2}+\sum_{t=2}^{T}\left\|p_{i}(t)-p_{i}(t-1)\right\|^{2} \\
& +\sum_{t=2}^{T} \sum_{j \in N_{i}(t)}\left(\left\|p_{i}(t)-p_{j}(t)\right\|-\left\|p_{i}(t-1)-p_{j}(t-1)\right\|\right)^{2} \\
& +\sum_{t=1}^{T-1} \sum_{j \in N_{i}(t)}\left(\left\|p_{i}(t)-p_{j}(t)\right\|-\left\|p_{i}(t+1)-p_{j}(t+1)\right\|\right)^{2}
\end{aligned}
$$

where $E_{d}$ is a data term that measures the closeness of the optimizing trajectory to the initially tracked trajectory. $E_{s}$ is a regularization term that measures smoothness of the trajectory of a node and its relative velocity to its neighbors. $\beta$ is a scalar for weighting different energy terms. We set $\beta=2$ in our fabric texture example (Figure 3.33-3.34). Note that $N_{i}(t)$ is the set of the four neighbors of node $i$; when a neighboring node is occluded, this neighbor is excluded in $N_{i}(t)$. We use the gradient-descent method to solve our optimization problem. Despite the large number of variables, the optimization process converges quickly since the initial condition $\left(p_{i}^{o}(t)=q_{i}(t) \forall i, t\right)$ is often close to the optimum. The video trousers_lattice_both.mp4 shows the lattice motion before and after smoothing.

Lighting Deformation Field Smoothing. The extracted lighting deformation field may be noisy at regions where the geometric distortion of textons are large or part of the textons are occluded [Liu et al., 2004]. Since we deal with video data, all lighting deformation fields in an image sequence can be utilized to repair the regions where the lighting deformation field is noisy or empty. We experimented with different 3D filtering approaches on a spatiotemporal volume formed by stacking all lighting deformation fields. We found that the median filtering is good enough for our application. Figure 3.31 shows an example of a lighting deformation field before and after smoothing. One can observe that there are some 'holes' in the lower right region of the un-smoothed lighting deformation fields (top row). The missing lighting information at hole regions is filled using the information from neighboring image frames in the temporal smoothing process. 

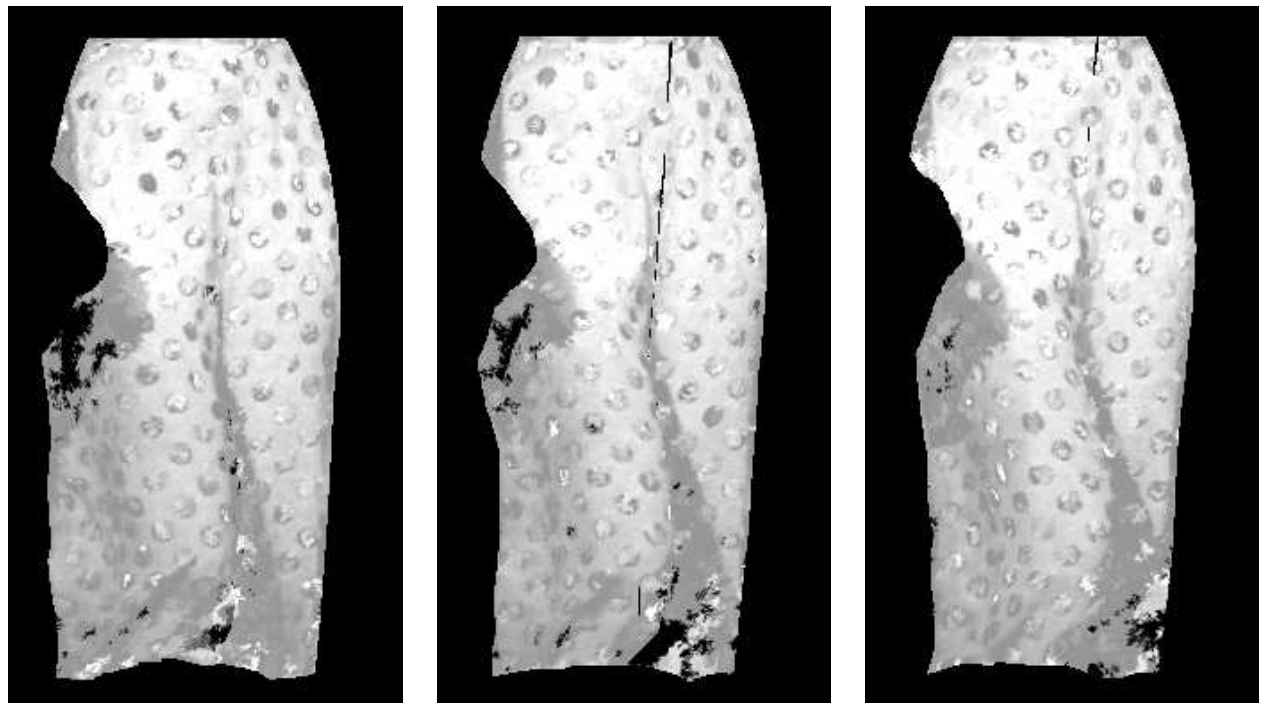

(a) Lighting DF without smoothing at frame 14, 15 and 16
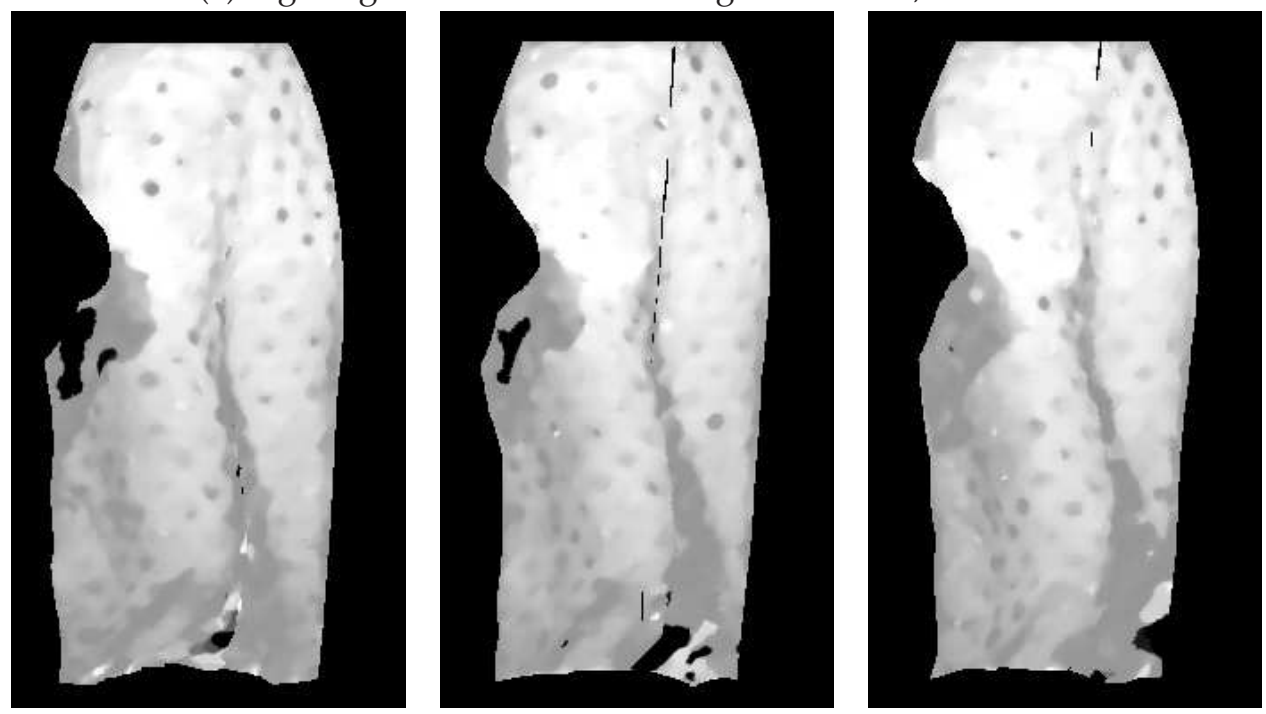

(b) Lighting DF with spatial smoothing only
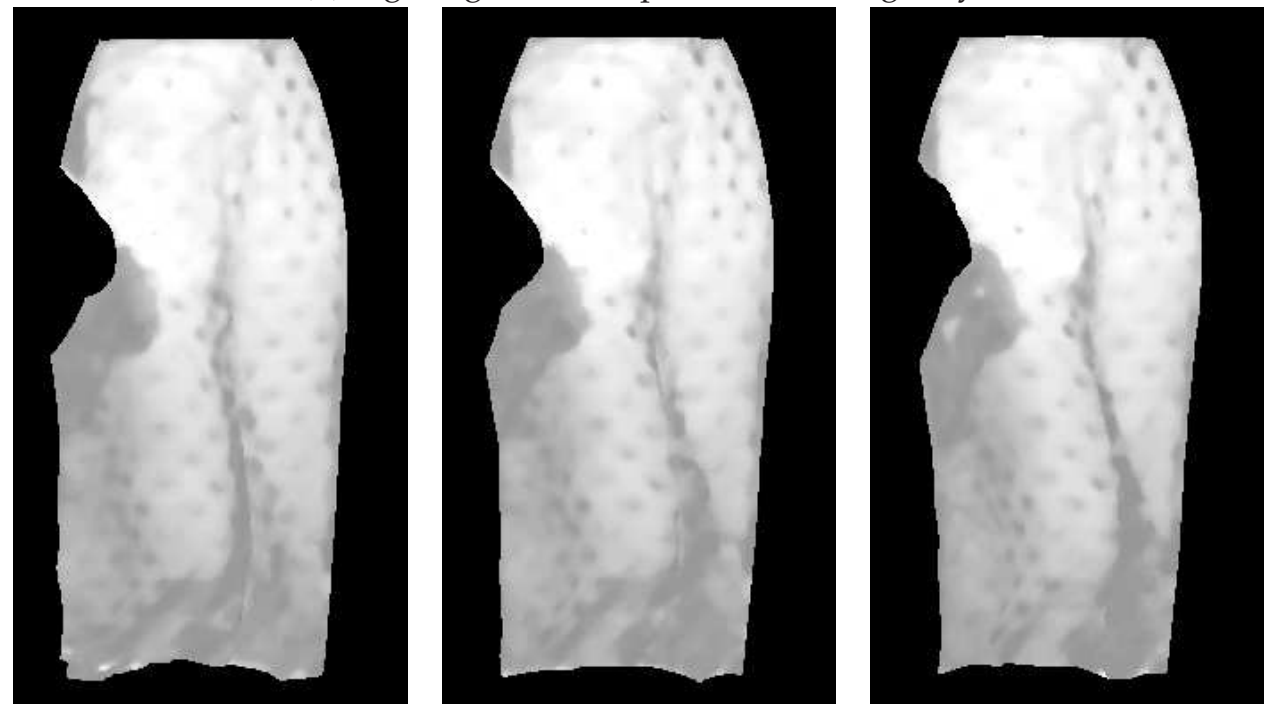

(c) Lighting DF with spatiotemporal smoothing

Figure 3.31. Comparison of lighting DF without and with smoothing. Note that only smoothing a light DF spatially cannot totally remove "holes" in the original DFs. 
Spatiotemporal Stitching. The geometric structure of an NRT on a complex surface usually cannot be represented by a single lattice. For instance, a fabric texture on clothes may be separated by seams between sleeve and shoulder or between left and right trousers. In these cases, it is necessary to connect two lattice structures at different regions. The boundary between two lattices, however, may not be obvious. It is not easy to track this boundary accurately in a video.

Instead of stitching two lattices directly, we stitch two image patches whose regions are defined by their respective lattices. We then apply dynamic programming in the spatiotemporal space to stitch two image patches of NRTs. Suppose $I_{1}(t)$ and $I_{2}(t)$ are two overlapping image patches of the textures we want to stitch at frame $t$, and $I(t)$ is the image patch after stitching, the cost function for dynamic programming to generate a path along $y$ direction at frame $t$ is defined as follows:

$$
\begin{aligned}
& c(t, x, y)=\alpha \cdot\left\|I_{1}(t, x, y)-I_{2}(t, x, y)\right\|+ \\
& \sum_{i=0}^{x-1}\left\|I_{1}(t, x-i, y)-I(t-1, x-i, y)\right\|+ \\
& w(t)-1 \\
& \sum_{i=1}^{\infty(t)-1}\left\|I_{2}(t, x+i, y)-I(t-1, x+i, y)\right\|
\end{aligned}
$$

where $(x, y)$ is the location of a pixel, and $\|\cdot\|$ is defined as the sum of intensity differences of all color channels. $w(t)$ is the width of the image patch $I_{1}(t)$ and $I_{2}(t)$. The first term in (3.23) penalizes the image difference spatially, and the second and third terms penalize the image difference temporally. $\alpha$ is a scalar to weight the influence of the spatial and temporal terms. In the trousers example (Figure 3.34), $\alpha=0.02$ is used. The temporal matching cost plays an important role in maintaining temporal coherence. If the temporal matching cost is not considered in dynamic programming, the generated stitching path could change abruptly between frames such that the temporal coherence is not maintained. See the video trousers_add_stitch.mp4 for a comparison for with and without spatiotemporal stitching.

Results. We tested our texture replacement approach on a underwater texture (Figure 3.32) and a fabric textures on trousers (Figure 3.33-3.34). In the video of underwater texture, although there are minor tracking errors at some frames, the computed geometric deformation field was able to capture most of the motion characteristics of the texture in the input video. The replacement results reflect the visual effects of a texture 
being seen through water. To extract the reflection highlights in the replacement results, we allow a larger range of lighting variations when computing the lighting distribution [Tsin et al., 2001]. That is, lighting samples that are normally considered as outliers are included to estimate the lighting distribution. Additionally, we apply a Gaussian filter to smooth the boundary of highlighted region to improve the replacement results. In the trousers video, we use an interactive rotoscoping program [Agarwala et al., 2004] to segment textured regions in the image sequence. To evaluate the effectiveness of our algorithm, we show the replacement results in different processing stages in the video trousers_processing.mp4.

\subsubsection{Video Superimposing}

Video superimposing puts augmented images on a moving object in a video. To obtain realistic visual effect, the augmented image should move or deform with the surface of the object. We can achieve superimposition by applying the captured geometric and lighting DFs to the augmented image. The process is the same as texture replacement except that we also need to apply the geometric DF to a segmentation map which defines the region of the augmented object, such as text region or shape of an object. The warped segmentation map is then applied to the warped augmented image to obtain the superimposed image. Figure 3.35(c) shows the results of superimposing text on a dress. Note that the letter ' $U$ ' preserves the self-occlusion effect in the output video. It is worth mentioning that superimposing in video was also demonstrated in [Bartoli et al., 2004; Pilet et al., 2005]. However, they do not handle occlusion nor preserve the photometric effects in the superimposed results.

\subsubsection{NRT Video Synthesis}

We develop a video synthesis algorithm that generates a video by image retrieval. The basic idea is to treat the tracked lattice motion in an input video as a motion field, where the motion field describes the displacements of the lattice points between two consecutive frames. This motion field characterizes the NRT at each image frame of the input video. We can then synthesize a new motion field and use the the synthesized motion field to retrieve images from the input video. The retrieving criterion is based on the similarity of motion. 

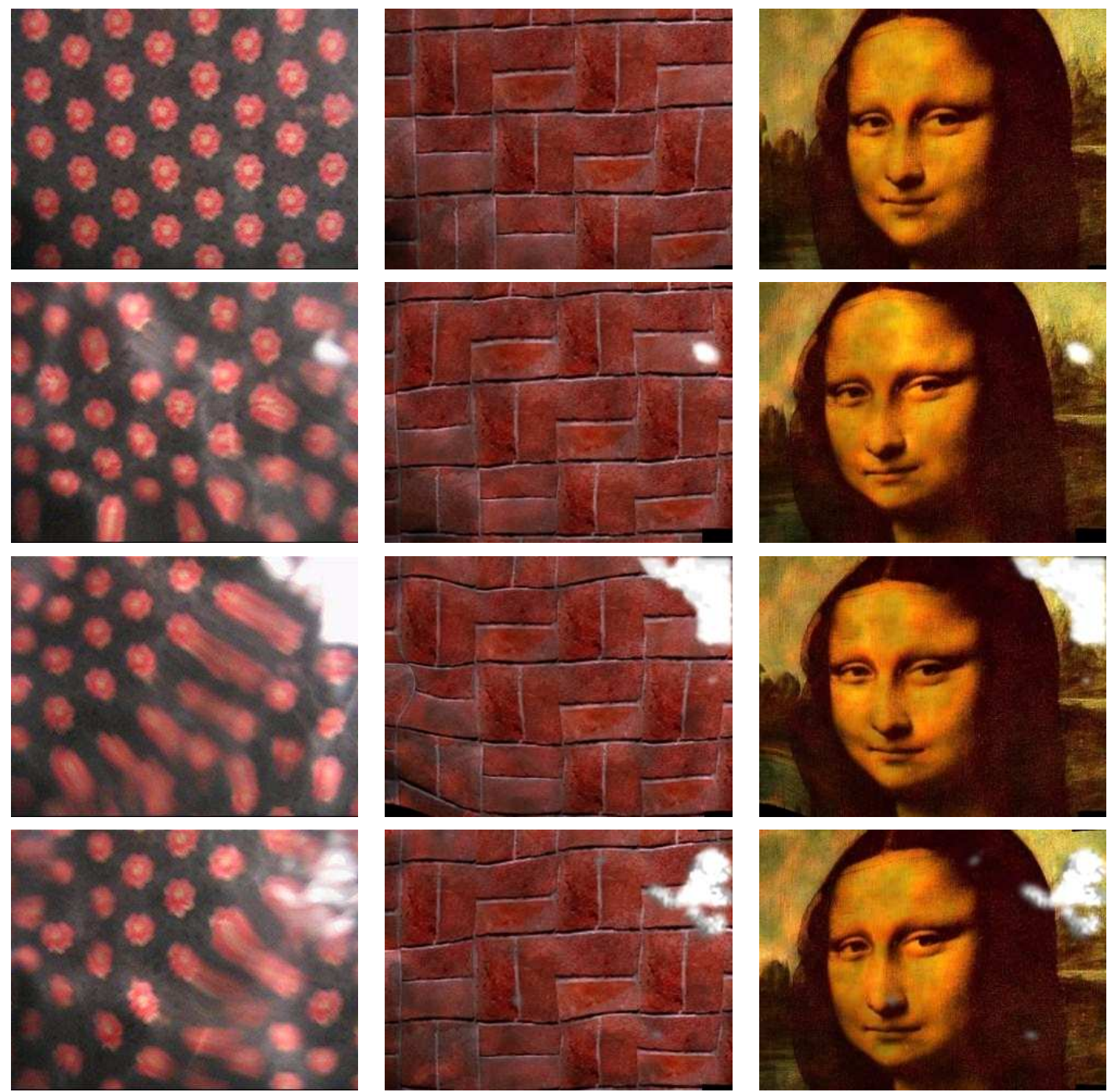

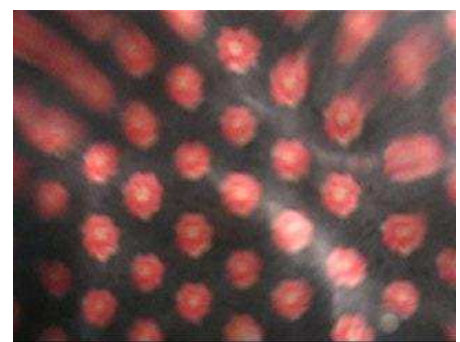

(a)

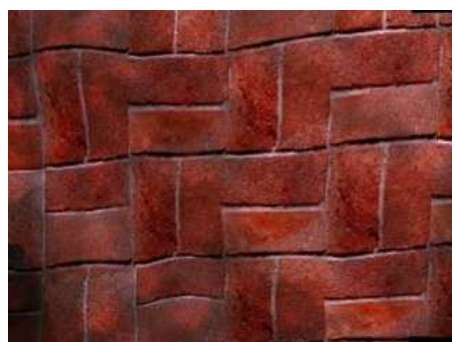

(b)

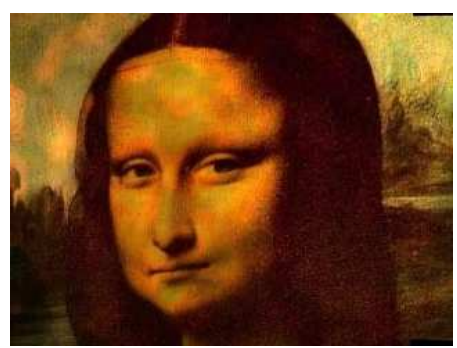

(c)

Figure 3.32. Texture replacement result of a pattern seen through disturbed water (water_replaced.mov). (a) Input video at frame 1,79, 82, 91 and 100. (b) Texture replacement with a brick texture. (c) Texture replacement with a Monalisa's image. 

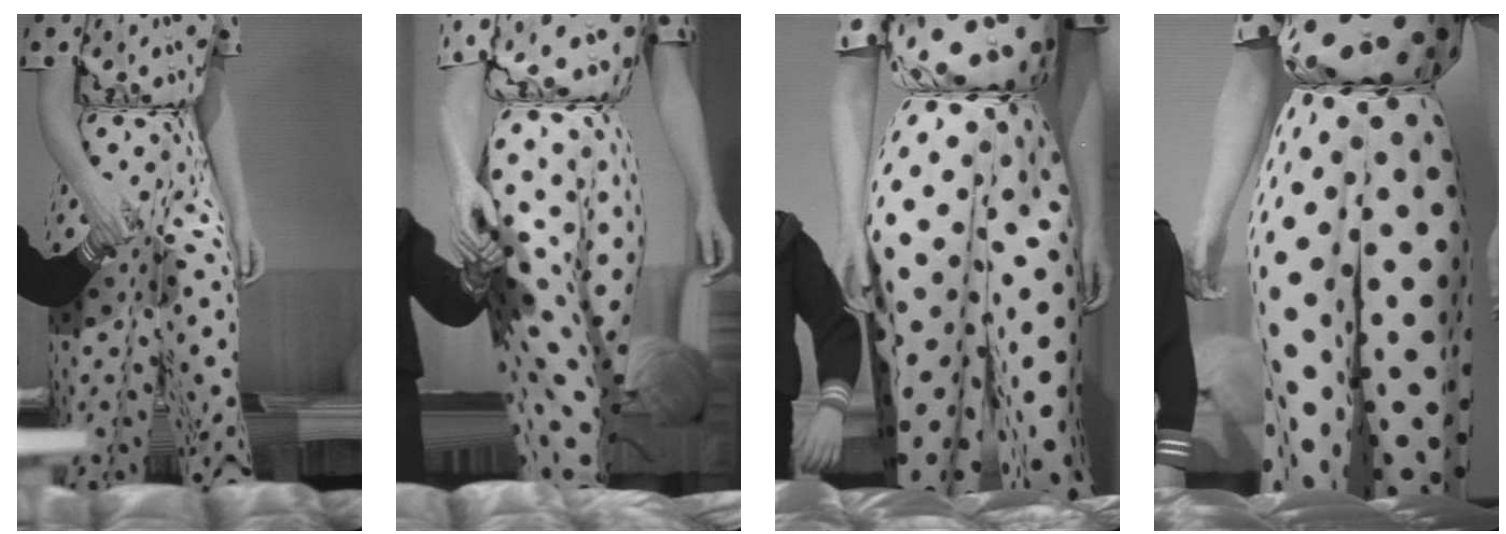

(a) Frame 1, 21, 41, and 61 of the input video (trousers_tracked.mov).
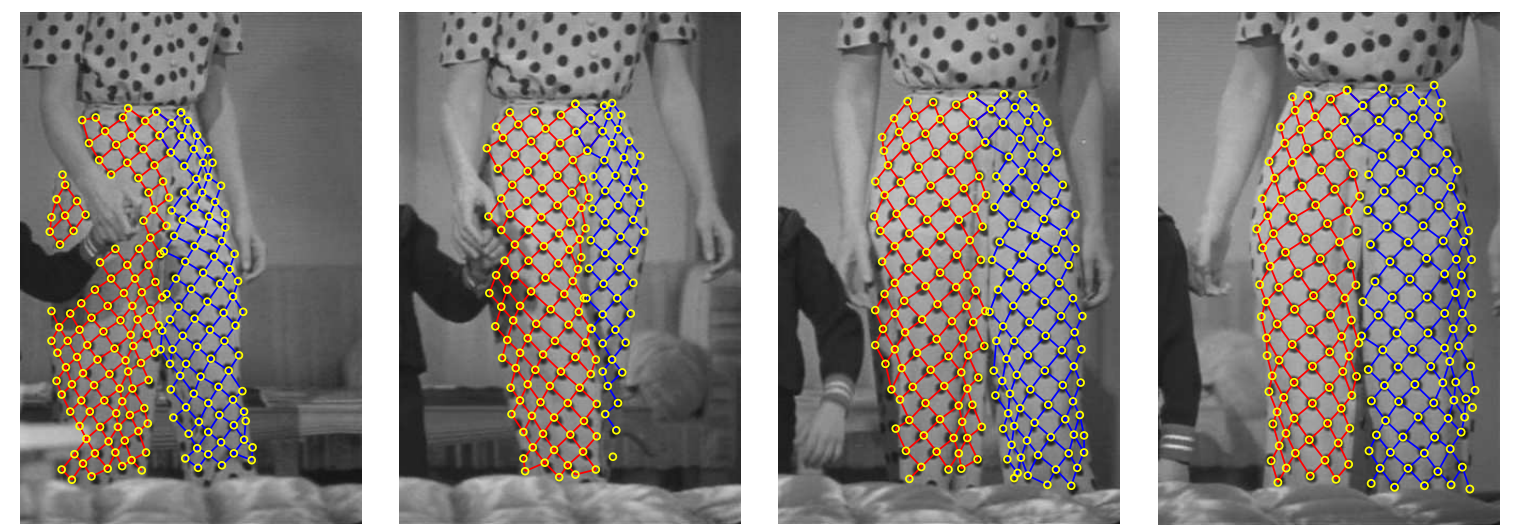

(b) Tracked lattices (trousers_tracked.mov).
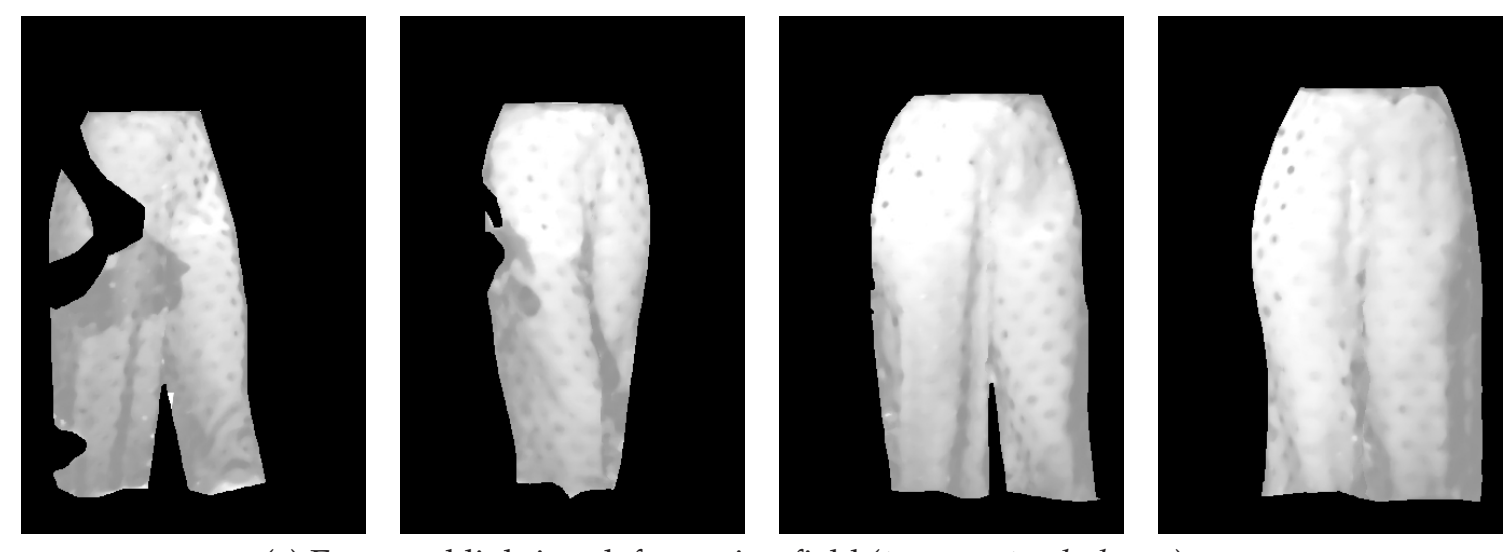

(c) Extracted lighting deformation field (trousers_tracked.mov).

Figure 3.33. Tracked lattices and extracted lighting DFs from a fabric texture.

Our video texture synthesis algorithm is basically an extension of the 2D geometric DF synthesis. Figure 3.36 shows the major components of our video texture synthesis algorithm: (1) dynamic NRT tracking, (2) 3D deformation field synthesis, and (3) video composing by image retrieval. The motion field captured by dynamic NRT tracking is a 

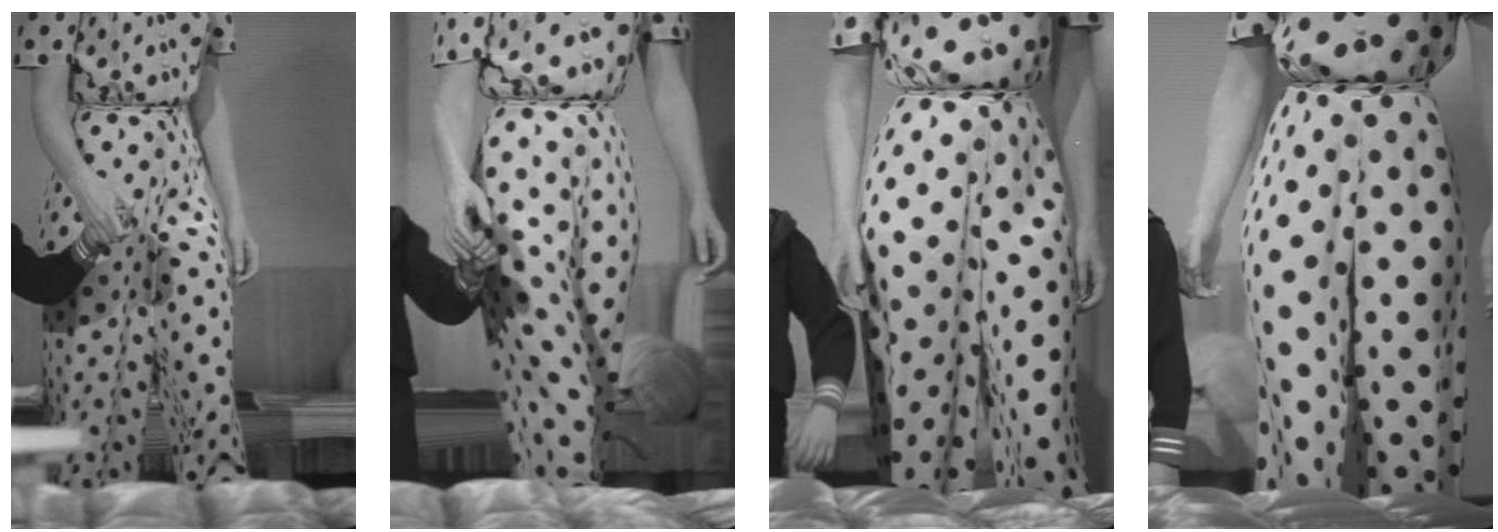

(a) Frame 1, 21, 41, and 61 of the input video (trousers_tracked.mov).
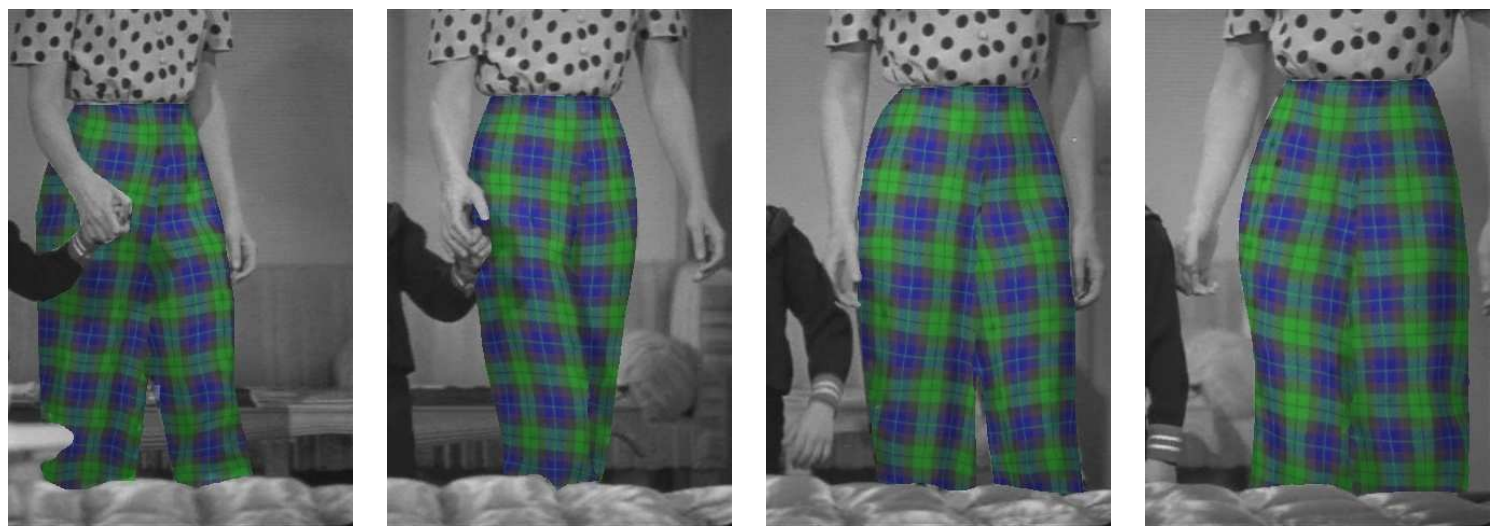

(b) Replaced by a tartan texture (trousers_replaced.mov).
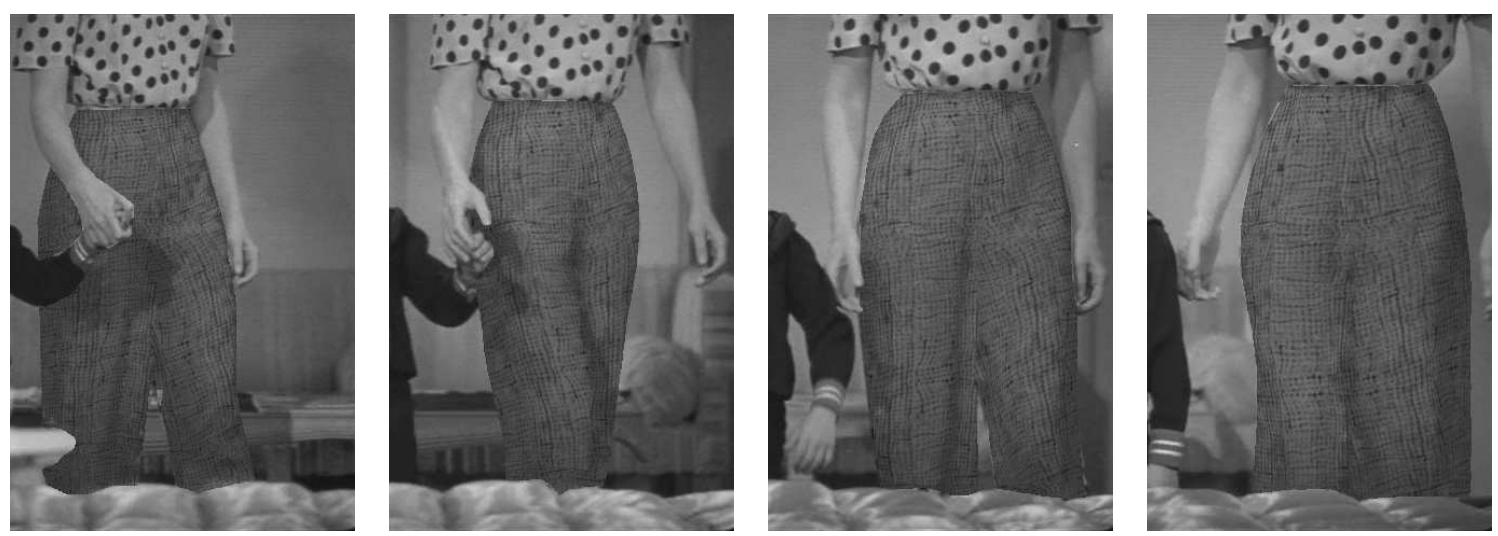

(c) Replaced by a gray-level texture (trousers_replaced.mov).

Figure 3.34. Texture replacement results of a fabric texture on trousers.

3D deformation field $d_{g e o}:(x, y, t) \rightarrow(d x, d y)$, which can be treated as a 3D texture. To synthesize this 3D deformation field, we modified the pixel-based texture synthesis algorithm [Efros and Leung, 1999] to handle 3D textures. We use a $5 \times 5 \times 5$ neighborhood and traverse in the scanline order in this $3 \mathrm{D}$ texture synthesis process. The position of 

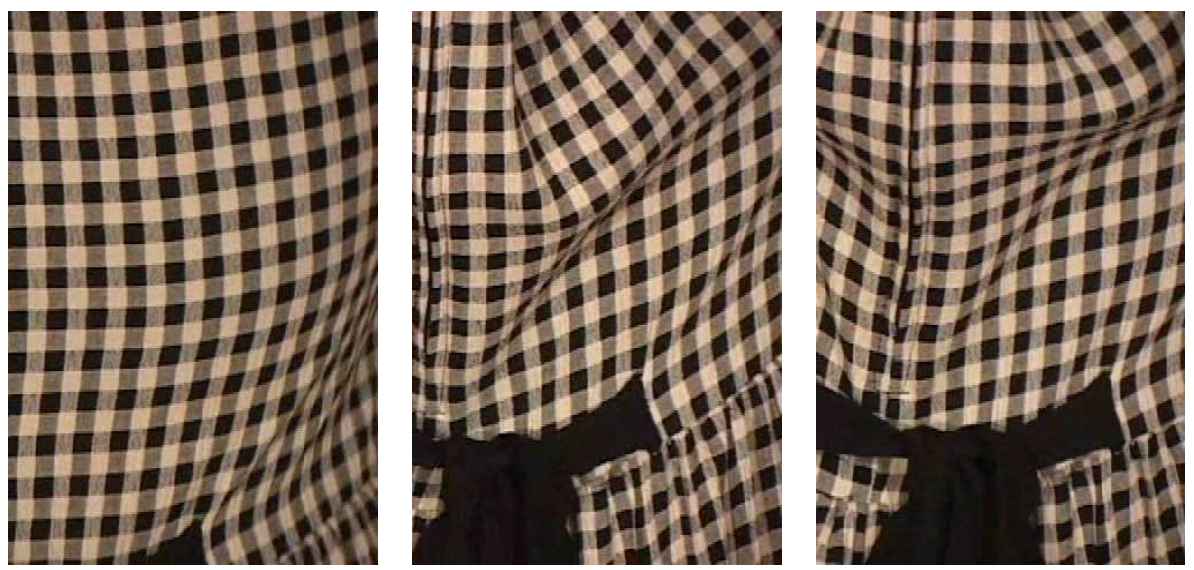

(a) Input video at frame 110, 180, and 230.
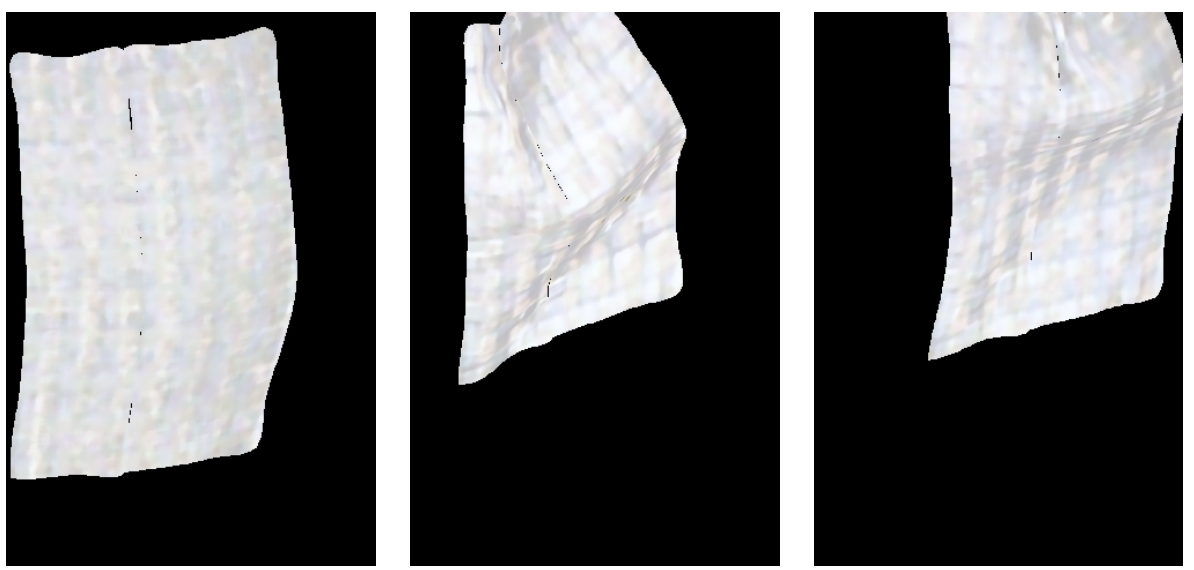

(b) Extracted lighting deformation field.
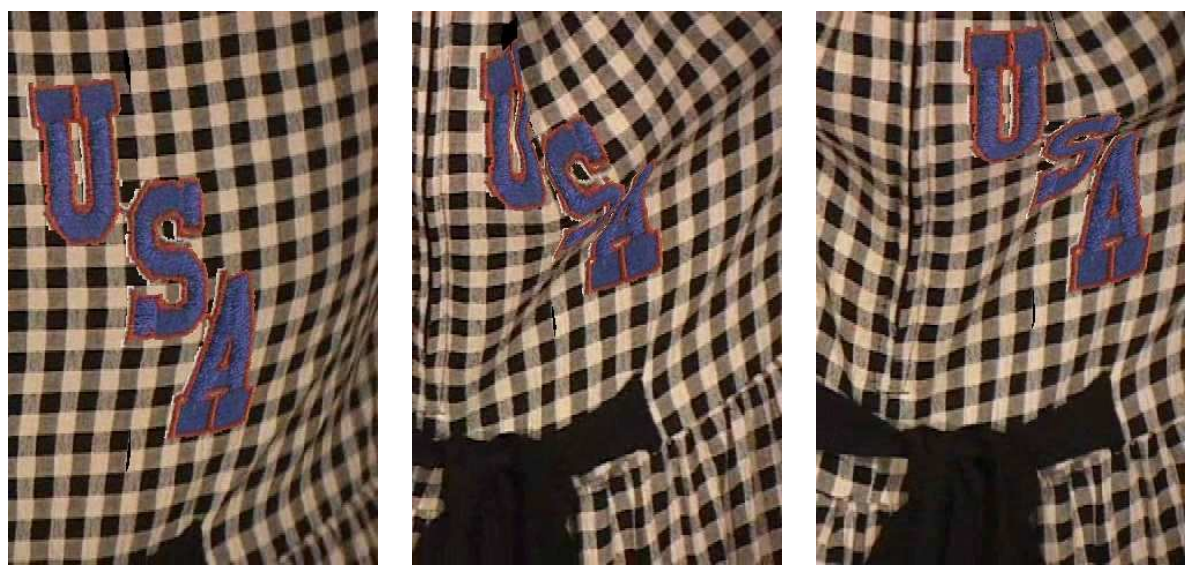

(c) Text superimposed video.

Figure 3.35. Video superimposing result under self-occlusion (superimpoing.avi). One can observe that the top-right corner of the letter ' $U$ ' preserves the occlusion effect in the input video. 


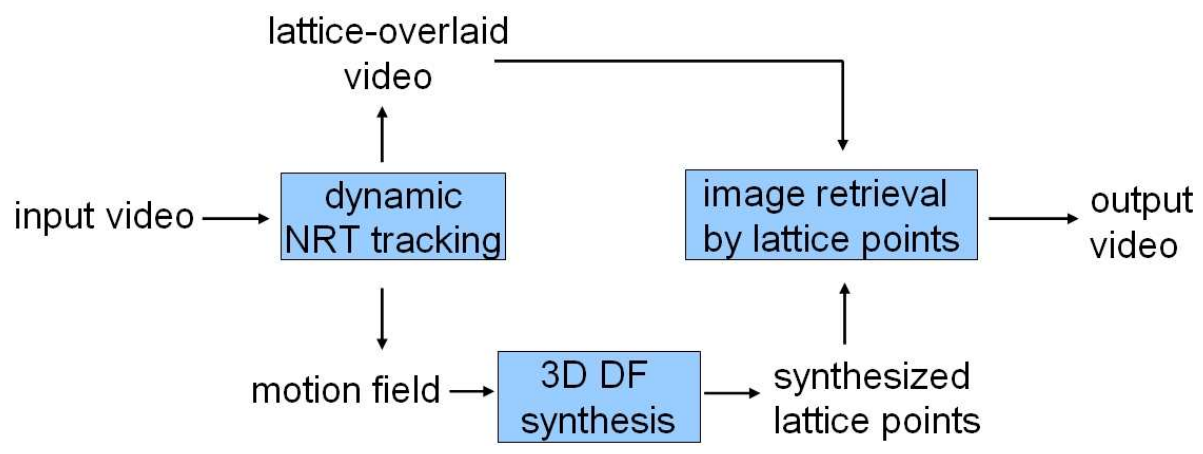

Figure 3.36. This figure shows an overview of video texture synthesis algorithm. The motion field of an input video is obtained by the dynamic NRT tracking algorithm. A new motion of lattice points is then generated by the 3D DF synthesis algorithm and is used to retrieve images from the input video. The retrieved images are warped and combined to generate the output video.

lattice points in each frame can be obtained by accumulating the displacements in the synthesized 3D deformation field.

The final step is to generate the video according to the positions of the lattice points in each frame. We treat the input image sequence as a rendering database. For each synthesized frame, we use the positions of lattice points to retrieve an image from the database. As the position data are represented in the global coordinate, we apply the rigid registration to adjust the difference of global position and orientation between the synthesized lattice points and those in the database. This will provide better image retrieval results. There are still residual errors between the positions of the synthesized (desired) lattice points and those in the retrieved images. We apply the MFFD algorithm to warp the retrieved images to correct these residual errors.

We used our algorithm to generate two synthesis results: (1) slow_syn.avi is directly synthesized from a video of slowly waving cloth, slow.avi(Figure 3.37); (2) mixed.avi is synthesized from a summed motion field of two videos, slow.avi and fast.avi(Figure 3.38). Although these two examples do not directly synthesize the image pixels at the bottom level, synthesizing videos in a higher level, like motion domain, has the advantage to manipulate data more flexibly and efficiently.

Our approach differs from other temporal texture synthesis algorithms [Wei and Levoy, 2000; Kwatra et al., 2003; Doretto et al., 2003] in that we do not directly synthesize image pixels or image patches; instead, we synthesize the motion of the lattice points in a 3D spatiotemporal space. Our approach is close to the video texture [Schödl 


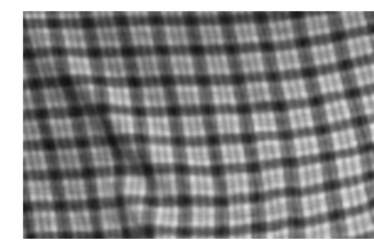

input video: slow.avi

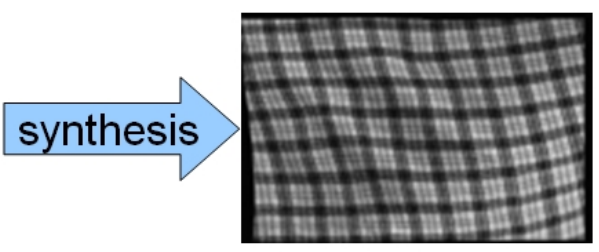

output video: slow_syn.avi

Figure 3.37. Dynamic NRT synthesis example 1: synthesizing from a single motion field.

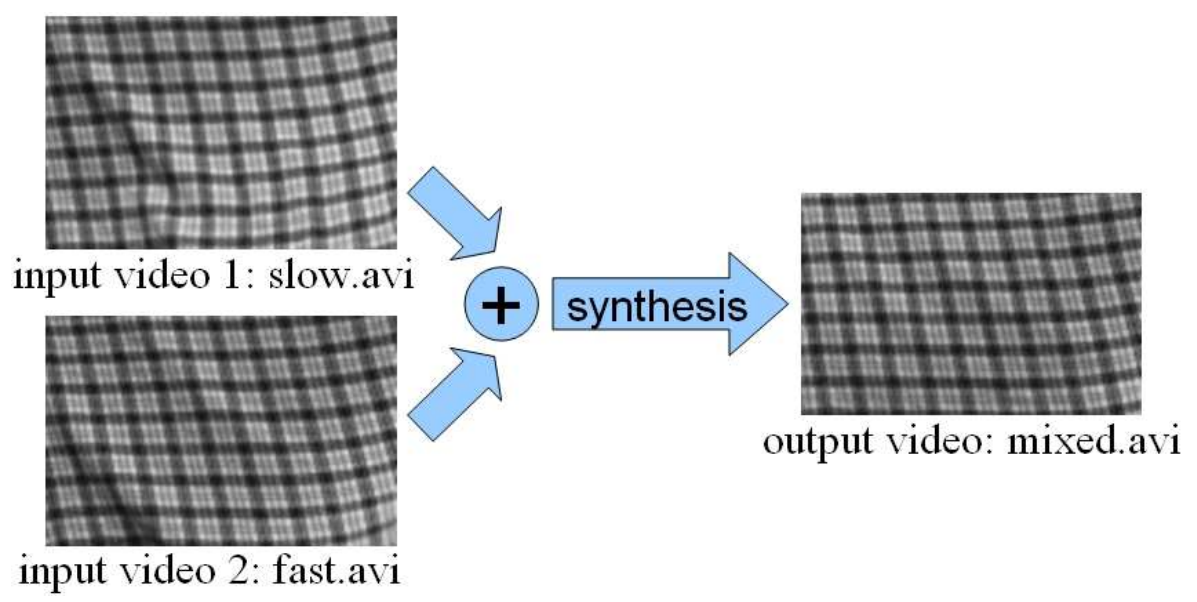

Figure 3.38. Dynamic NRT synthesis example 2: synthesizing from two motion fields by summation.

et al., 2000] in that both approaches retrieve whole images from the input video; however, we use motion similarity to retrieve images while the video texture approach uses similarity of image pixels to retrieve images. 


\section{CHAPTER 4}

\section{Conclusion}

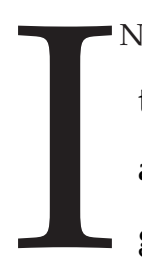

$\mathrm{N}$ this thesis, we propose a lattice-based MRF model for dynamic near-regular textures. We treat textons of a dynamic NRT as separate moving objects with a topological constraint while allowing individual textons to vary flexibly in geometry and appearance. Our lattice-based MRF model consists of a lattice structure model that characterizes the topological constraint of a dynamic NRT and a registration-based image observation model that handles the geometry and appearance variations of individual textons. Under such a computational modeling of the topology and appearance of a dynamic NRT, we treat dynamic NRT tracking as a spatiotemporal inference problem and apply the belief propagation and the particle filtering algorithms to solve it. We demonstrate the effectiveness of our algorithm on tracking dynamic NRTs under rapid movements, occlusions or illumination changes through different mediums. Our manipulation algorithm handles the temporal coherence problem by spatiotemporal deformation field smoothing and spatiotemporal stitching. We demonstrate applications of our algorithm in texture replacement and superimposition on real videos.

\subsection{Contributions}

This thesis has three major contributions. First, the major contribution is a latticebased MRF formalization for dynamic NRT modeling. Our lattice-based MRF model integrates a high-level topological structure model and a low-level registration-based texton geometry and appearance model. Under such formalization, we are able to characterize the lattice topology and the geometry and appearance of individual textons of a 
dynamic NRT. Secondly, we implement a dynamic NRT tracking algorithm that can effectively handle ambiguous correspondences, occlusions, illumination variations, and appearance variations. Thirdly, the proposed dynamic NRT manipulation framework makes it possible to replace a dynamic NRT or superimpose on a dynamic NRT in real videos. Our contribution in dynamic NRT opens up many novel applications in computer vision and computer graphics.

\subsection{Limitations}

\subsubsection{Static NRT analysis and manipulation}

The synthesis and manipulation results, and the NRT synthesis comparison study demonstrate the effectiveness of the algorithm. However, there are still some limitations of the algorithm. First, user intervention is needed for identifying the underlying lattice of an NRT. An automatic lattice extraction algorithm will greatly facilitate the process of NRT analysis and manipulation. Second, the lighting deformation field cannot capture lighting effects on a non-lambertian surface. Third, only attached shadows that are directly caused by surface geometry can be captured and synthesized using image analogies. Cast shadows from non-local regions may cause un-reasonable (unexplainable) synthesized results.

The user evaluation conducted in this comparison study is still preliminary in terms of evaluation criteria. In our future study, we would like to design more detailed evaluation criteria so that we can understand which factor affects the human perception more on synthetic textures. For example, we can divide the evaluation criteria into several items, such as the faithfulness of the color/intensity of a synthetic texture, and the faithfulness of the geometric structure of a synthetic texture. We can also apply user evaluation results to verify or adjust the definition of ga-scores. We believe that a thorough comparison study can lead to better understanding of NRTs and benefit the development of NRT synthesis algorithms.

\subsubsection{Dynamic NRT Modeling, Tracking and Manipulation}

The proposed lattice-based MRF model makes our tracking algorithm capable of tracking varied types of dynamic textures under rapid motion, folding motion or occlusion. There are several issues that remain to be studied in the future. First, we currently 
assume that the topology of a texture does not change; however, in crowd motion, for example, the connection topology among individuals may actually change from time to time. It would be better to allow the topology to adapt during the tracking process. A possible direction is to adopt a dynamic Bayesian network [Murphy, 2002] to model a varying topology. Second, we would like to extend our MRF to model a folding topology. Third, it is possible to combine a shape-from-texture algorithm to obtain the 3D geometry, which will further expand the applications of NRT manipulation, such as lighting and viewing angle variation.

\subsection{Potential Applications and Future Extensions}

There are several potential applications of this work. One of them is a virtual fitting room. A customer can upload his/her photo or video wearing any near-regular textured cloth, and the virtual fitting room can generate an image or video in which the customer wears the cloth of his/her choice. Another application may be fashion design preview. A designer can preview the visual effects of different fabric textures on the same dress using a prerecorded footage of a model.

For dynamic NRT manipulation, more applications can be explored by synthesizing or manipulating a sequence of DFs. For example, it is possible to extend the size and length of videos by synthesizing geometric and lighting deformation fields in the temporal and spatial domains. We can treat the geometric deformation field as a 3D texture and synthesize it. The synthesized 3D deformation field then can be used to synthesize associated lighting deformation field using deformation field analogy (section 2.3.3).

Several research issues can be further explored in the future. First, how do we infer the folding topology of a lattice in occluded regions? This will provide a better model for dynamic NRTs. Second, how do we synthesize a dynamic NRT under occlusion? The visibility map can be considered as an additional deformation field that is associated with the geometric and lighting DFs. A synthesis scheme like deformation field analogy needs to be developed to assure that the association among geometry, lighting and occlusion is maintained in the synthesized result. Also, the temporal coherence needs to be handled in the synthesized videos. Third, can we reconstruct the surface geometry using the tracking result from a single-view video? It is possible to apply a shape from texture algorithm to achieve this goal; however, when the textured surface 


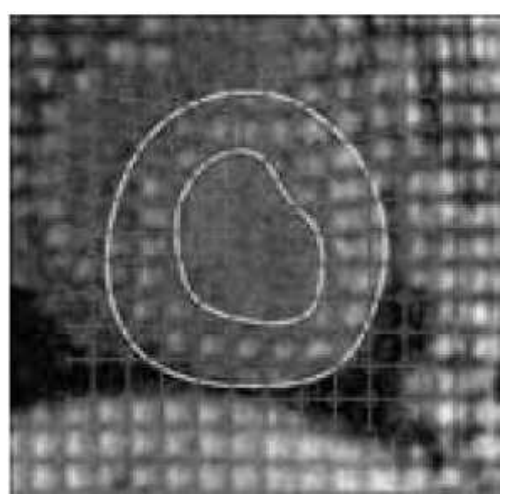

Figure 4.1. It is possible to apply our tracking algorithm to other types of data that demonstrate lattice property. This figure shows a tagged MRI image of a heart. Those tagged lines in the image form a lattice structure.

is folded or the texton density on the surface is sparse, how do we obtain a reasonable result? Fourth, although we only deal with image textures in this thesis, is it possible to extend this work to handle other types of data? Or can we transform the data into a near-regular form so that our algorithm can be used? Figure 4.1 shows an MRI image of a heart where an MRI imaging technique called "tagging" is used to generate tagged lines in the image. These tagged lines can be used to track and measure heart motion. It is possible to apply our tracking algorithm to tagged MRI data for heart motion tracking.

\subsection{Summary}

Near-regular textures play an important role on the texture spectrum and our daily life, but they have rarely been addressed in computer vision and computer graphics in the past. This thesis extends our previous work on static NRT analysis and manipulation to dynamic NRT modeling, tracking and manipulation. The proposed framework provides a feasible solution to this problem. This is just the starting point of the research on dynamic NRTs. By introducing dynamic NRT modeling and tracking to the computer vision community and dynamic NRT manipulation to the computer graphics community, we hope our work will draw more research interests on NRTs in the near future. 


\section{BIBLIOGRAPHY}

Agarwala, A., Hertzmann, A., Salesin, D. H., and Seitz, S. M. [2004]. Keyframe-based tracking for rotoscoping and animation. In ACM SIGGRAPH, pages 584-591.

Anderson, B. and Moore, J. [1979]. Optimal Filtering. New Jersey: Prentice Hall.

Ashikhmin, M. [2001]. Synthesizing natural textures. In Symposium on Interactive 3D Graphics, pages 217-226.

Baker, S. and Matthews, I. [2004]. Lucas-kanade 20 years on: A unifying framework. International Journal of Computer Vision, 56(3), 221-255.

Bar-Joseph, Z., El-Yaniv, R., Lischinski, D., and Werman, M. [2001]. Texture mixing and texture movie synthesis using statistical learning. IEEE Transactions on Visualization and Computer Graphics, 7(2), 120-135.

Bar-Shalom, Y. [1987]. Tracking and data association. Academic Press Professional, Inc.

Bartoli, A., von Tunzelmann, E., and Zisserman, A. [2004]. Augmenting images of nonrigid scenes using point and curve correspondences. In CVPR, pages 699-706.

Bhat, K., Seitz, S., Hodgins, J., and Khosla, P. [2004]. Flow-based video synthesis and editing. ACM Transactions on Graphics, 23(3), 360-363.

Black, M. J. and Anandan, P. [1996]. The robust estimation of multiple motions: Parametric and piecewise-smooth flow fields. Computer Vision and Image Understanding, 63(1), 75-104.

Blackman, S. and Popoli, R. [1999]. Design and analysis of modern tracking system. Artech House.

Bonet, J. S. D. [1997]. Multiresolution sampling procedure for analysis and synthesis of texture images. In ACM SIGGRAPH, pages 361-368.

Burgi, P.-Y., Yuille, A. L., and Grzywacz, N. M. [2000]. Probabilistic motion estimation based on temporal coherence. Neural Computation, 12(8), 1839-1867. 
Chantler, M. and Gool, L. V. (Eds.). [2005]. International Journal of Computer Vision: Special Issue on Texture Analysis and Synthesis, volume 62. Springer.

Chetverikov, D. and Péteri, R. [2005]. A brief survey of dynamic texture description and recognition. In International Conference on Computer Recognition Systems, Advances in Soft Computing, pages 17-26, Rydzyna, Poland. Springer-Verlag.

Choi, K.-J. and Ko, H.-S. [2002]. Stable but responsive cloth. In SIGGRAPH 2002, pages 604-611, New York, NY, USA. ACM Press.

Cootes, T. F., Edwards, G. J., and Taylor, C. J. [1998]. Active appearance models. In ECCV, pages 484-498.

Cox, I. [1993]. A review of statistical data association techniques for motion correspondence. International Journal of Computer Vision, 10(1), 53-66.

Coxeter, H. S. M. and Moser, W. O. J. [1979]. Generators and relations for discrete groups (4 ed.). New York: Springer-Verlag.

Doretto, G., Chiuso, A., Wu, Y. N., and Soatto, S. [2003]. Dynamic textures. International Journal of Computer Vision, 51(2), 91-109.

Dorsey, J., Edelman, A., Jensen, H. W., Legakis, J., and Pedersen, H. K. [1999]. Modeling and rendering of weathered stone. In ACM SIGGRAPH, pages 225-234.

Doucet, A., Freitas, N. D., and Gordon, N. [2001]. Sequential Monte Carlo Methods in Practice. Springer-Verlag.

Ebert, D. S., Musgrave, F. K., Peachey, D., Perlin, K., and Worley, S. [2002]. Texturing and Modeling: A Procedural Approach (3rd ed.). Morgan Kaufmann.

Efros, A. A. and Freeman, W. T. [2001]. Image quilting for texture synthesis and transfer. In ACM SIGGRAPH, pages 341-346.

Efros, A. A. and Leung, T. K. [1999]. Texture synthesis by non-parametric sampling. In $\operatorname{ICCV}(2)$, pages 1033-1038.

Fang, H. and Hart, J. C. [2004]. Textureshop: Texture synthesis as a photograph editing tool. In ACM SIGGRAPH, pages 354-359.

Fedorov, E. S. [1885]. The elements of the study of figures. In Zapiski Imperatorskogo S. Peterburgskogo Mineralogichesgo Obshchestva [Russian][Proc. S. Peterb. Mineral. Soc.], volume 21, pages 1-289.

Forsyth, D. A. [2002]. Shape from texture without boundaries. In ECCV, pages 225-239. 
Freeman, W. T., Pasztor, E. C., and Carmichael, O. T. [2000]. Learning low-level vision. Int. J. Comput. Vision, 40(1), 25-47.

Grünbaum, B. and Shephard, G. C. [1987]. Tilings and Patterns. W. H. Freeman and Company, New York.

Guskov, I. [2002]. Efficient tracking of regular patterns on non-rigid geometry. In Proceedings of ICPR.

Guskov, I. [2004]. Multiscale inverse compositional alignment for subdivision surface maps. In ECCV (1), pages 133-145.

Guskov, I., Klibanov, S., and Bryant, B. [2003]. Trackable surfaces. In ACM Symposium on Computer Animation, pages 251-257.

Heeger, D. J. and Bergen, J. R. [1995]. Pyramid-based texture analysis/synthesis. In ACM SIGGRAPH, pages 229-238.

Hertzmann, A., Jacobs, C. E., Oliver, N., Curless, B., and Salesin, D. H. [2001]. Image analogies. In ACM SIGGRAPH, pages 327-340.

House, D. H. and Breen, D. E. (Eds.). [2000]. Cloth Modeling and Animation. Natick, Massachusetts: A.K. Peters, Ltd.

Isard, M. [2003]. Pampas: real-valued graphical models for computer vision. In CVPR, pages 613-620.

Isard, M. and Blake, A. [1998]. Condensation - conditional density propagation for visual tracking. Int. J. Comput. Vision, 29(1), 5-28.

Julesz, B. [1962]. Visual pattern discrimination. IRE Trans. on Information Theory, 8(2), 84-92.

Julesz, B. [1981]. Textons, the elements of texture perception and their interactions. Nature, 290, 91-97.

Kalman, R. E. [1960]. A new approach to linear filtering and prediction problems. Transactions of the ASME-Journal of Basic Engineering, 82(Series D), 35-45.

Khan, Z., Balch, T., and Dellaert, F. [2004]. An mcmc-based particle filter for tracking multiple interacting targets. In ECCV, pages 279-290.

Kwatra, V., Essa, I., Bobick, A., and Kwatra, N. [2005]. Texture optimization for example-based synthesis. ACM Transactions on Graphics, SIGGRAPH 2005, 24(3), 795802. 
Kwatra, V., Schödl, A., Essa, I., Turk, G., and Bobick, A. [2003]. Graphcut textures: Image and video synthesis using graph cuts. In ACM SIGGRAPH, pages 277-286.

Lee, S.-Y., Chwa, K.-Y., and Shin, S. Y. [1995]. Image metamorphosis using snakes and free-form deformations. In ACM SIGGRAPH, pages 439-448.

Lefebvre, S. and Hoppe, H. [2005]. Parallel controllable texture synthesis. ACM Transactions on Graphics (SIGGRAPH 2005), 24(3), 777-786.

Li, S. Z. [2001]. Markov Random Field Modeling in Image Analysis (2 ed.). Springer.

Liang, L., Liu, C., Xu, Y., Guo, B., and Shum, H. [2001a]. Real-time texture synthesis by patch-based sampling. ACM Transactions on Graphics, 20(3), 127-150.

Liang, L., Liu, C., Xu, Y., Guo, B., and Shum, H. [2001b]. Real-time texture synthesis by patch-based sampling. Technical Report MSR-TR-2001-40, Microsoft Research.

Lin, W.-C., Hays, J., Wu, C., Kwatra, V., and Liu, Y. [2004]. A comparison study of four texture synthesis algorithms on regular and near-regular textures. Technical Report CMU-RI-TR-04-01, Robotics Institute, Carnegie Mellon University.

Liu, Y. and Lin, W.-C. [2003]. Deformable texture: the irregular-regular-irregular cycle. In The 3rd International Workshop on Texture Analysis and Synthesis (Texture 2003), pages $65-70$.

Liu, Y., Lin, W.-C., and Hays, J. [2004]. Near-regular texture analysis and manipulation. In ACM SIGGRAPH, pages 368-376.

Liu, Y. and Tsin, Y. [2002]. The promise and perils of near-regular texture. In The 2nd International Workshop on Texture Analysis and Synthesis (Texture 2002), pages 77-81.

Liu, Y., Tsin, Y., and Lin, W.-C. [2005]. The promise and perils of near-regular texture. International Journal of Computer Vision, 62(1-2), 145-159.

Lobay, A. and Forsyth, D. A. [2004]. Recovering shape and irridiance maps from rich dense texton fields. In CVPR, pages 400-406.

Lucas, B. and Kanade, T. [1981]. An iterative image registration technique with an application to stereo vision. In IJCAI81, pages 674-679.

Matthews, I. and Baker, S. [2004]. Active appearance models revisited. International Journal of Computer Vision, 60(2), 135 - 164.

Matthews, I., Ishikawa, T., and Baker, S. [2004]. The template update problem. IEEE Transactions on Pattern Analysis and Machine Intelligence, 26(6), 810 - 815. 
Matusik, W., Zwicker, M., and Durand, F. [2005]. Texture design using a simplicial complex of morphable textures. ACM Transactions on Graphics (SIGGRAPH 2005), 24(3), 787-794.

Maurer, T. and von der Malsburg, C. [1996]. Tracking and learning graphs and pose on image sequences of faces. In 2nd International Conference on Automatic Face and Gesture Recognition (FG'96), pages 176-181.

McKee, S. P. and Welch, L. [1985]. Sequential recruitment in the discrimination of velocity. Journal of the Optical Society of America A, 2(2), 243-251.

Murphy, K. [2002]. Dynamic Bayesian Networks: Representation, Inference and Learning. $\mathrm{PhD}$ thesis, UC Berkeley.

Nealen, A. and Alexa, M. [2003]. Hybrid texture synthesis. In Eurographics Symposium on Rendering, pages 97-105.

Nelson, R. C. and Polana, R. [1992]. Qualitative recognition of motion using temporal texture. CVGIP Image Understanding, 56(1), 78-89.

Neter, J., Kutner, M. H., Wasserman, W., and Nachtsheim, C. J. [1996]. Applied Linear Statistical Models (4th ed.). McGraw-Hill.

Oh, B. M., Chen, M., Dorsey, J., and Durand, F. [2001]. Image-based modeling and photo editing. In ACM SIGGRAPH, pages 433-442.

Papoulis, A. and Pillai, S. U. [2002]. Probability, random variables, and stochastic processes (4 ed.). McGraw-Hill.

Pearl, J. [1998]. Probabilistic Reasoning in Intelligent Systems: Networks of Plausible Inference (2nd ed.). Morgan Kaufmann.

Perlin, K. [1985]. An image synthesizer. In ACM SIGGRAPH, pages 287-296.

Pilet, J., Lepetit, V., and Fua, P. [2005]. Real-time non-rigid surface detection. In CVPR, pages $822-828$.

Portilla, J. and Simoncelli, E. P. [2000]. A parametric texture model based on joint statistics of complex wavelet coefficients. International Journal of Computer Vision, 40(1), $49-71$.

Pritchard, D. and Heidrich, W. [2003]. Cloth motion capture. In Eurographics.

Provot, X. [1995]. Deformation constraints in a mass-spring model to describe rigid cloth behavior. In Graphics Interface '95, pages 147-154. 
Ramachandran, V. S. and Anstis, S. [1983]. Extrapolation of motion path in human visual perception. Vision Research, 23(1), 83-85.

Rao, A. and Lohse, G. [1993]. Identifying high level features of texture perception. CVGIP: Image Processing, 55, 218-233.

Saisan, P., Doretto, G., Wu, Y. N., and Soatto, S. [2001]. Dynamic texture recognition. In CVPR, volume 2, pages 58-63, Kauai, Hawaii.

Schattschneider, D. [1978]. The plane symmetry groups: their recognition and notation. American Mathematical Monthly, 85, 439-450.

Schödl, A. and Essa, I. [2002]. Controlled animation of video sprites. In ACM Symposium on Computer animation, pages 121-127.

Schödl, A., Szeliski, R., Salesin, D. H., and Essa, I. [2000]. Video textures. In ACM SIGGRAPH, pages 489-498.

Scholz, V. and Magnor, M. [2004]. Cloth motion from optical flow. In Proceedings of 9th International Fall Workshop on Vision, Modeling and Visualization.

Scholz, V., Stich, T., Keckeisen, M., Wacker, M., and Magnor, M. [2005]. Garment motion capture using color-coded patterns. In Eurographics.

Sclaroff, S. and Isidoro, J. [1998]. Active blobs. In ICCV, pages 1146-1153.

Sudderth, E., Ihler, A., Freeman, W., and Willsky, A. [2003]. Nonparametric belief propagation. In CVPR, pages 605-612.

Sudderth, E. B., Mandel, M. I., Freeman, W. T., and Willsky, A. S. [2004]. Distributed occlusion reasoning for tracking with nonparametric belief propagation. In Neural Information Processing Systems, pages 1369-1376.

Szummer, M. and Picard, R. W. [1996]. Temporal texture modeling. In IEEE Int. Conf. On Image Processing, volume 3, pages 823-826.

Tomasi, C. and Manduchi, R. [1998]. Bilateral filtering for gray and color images. In Proceedings of the Sixth International Conference on Computer Vision, page 839.

Tsin, Y., Liu, Y., and Ramesh, V. [2001]. Texture replacement in real images. In IEEE Conference on Computer Vision and Pattern Recognition, pages 539-544.

Turk, G. [1991]. Generating textures for arbitrary surfaces using reaction-diffusion. In ACM SIGGRAPH, pages 289-298.

Turk, G. [1994]. Texturing Surfaces Using Reaction-Diffusion. PhD thesis, University of North Carolina at Chapel Hill. 
Vidal, R. and Ravichandran, A. [2005]. Optical flow estimation and segmentation of multiple moving dynamic textures. In CVPR, pages 516-521.

Wang, Y. and Zhu, S. C. [2004]. Analysis and synthesis of textured motion: Particles and waves. IEEE Trans. Pattern Anal. Mach. Intell., 26(10), 1348-1363.

Watamaniuk, S. N., McKee, S. P., and Grzywacz, N. M. [1995]. Detecting a trajectory embedded in random-direction motion noise. Vision Research, 35(1), 65-77.

Watamaniuk, S. N. and Sekuler, R. [1992]. Temporal and spatial integration in dynamic random-dot stimuli. Vision Research, 32(12), 2341-2347.

Wei, L.-Y. and Levoy, M. [2000]. Fast texture synthesis using tree-structured vector quantization. In ACM SIGGRAPH, pages 479-488.

Wei, L.-Y. and Levoy, M. [2001]. Texture synthesis over arbitrary manifold surfaces. In ACM SIGGRAPH, pages 355-360.

Weiss, Y. and Freeman, W. [2001]. Correctness of belief propagation in gaussian graphical models of arbitrary topology. Neural Computation, 13(10), 2173-2200.

Wiskott, L., Fellous, J.-M., Krüger, N., and von der Malsburg, C. [1997]. Face recognition by elastic bunch graph matching. IEEE Trans. Pattern Anal. Mach. Intell., 19(7), 775779.

Witkin, A. and Kass, M. [1991]. Reaction-diffusion textures. In ACM SIGGRAPH, pages 299-308.

$\mathrm{Wu}, \mathrm{Q}$. and $\mathrm{Yu}, \mathrm{Y}$. [2004]. Feature matching and deformation for texture synthesis. ACM Transactions on Graphics (SIGGRAPH 2004), 23(3), 364-367.

Yedidia, J., Freeman, W., and Weiss, Y. [2001]. Understanding belief propagation and its generalizations. In International Joint Conference on Artificial Intelligence.

Yokoyama, R. and Haralick, R. [1979]. Texture pattern image generation by regular markov chain. Pattern Recognition, 11(4), 225-233.

$\mathrm{Yu}, \mathrm{T}$. and $\mathrm{Wu}, \mathrm{Y}$. [2005]. Decentralized multiple target tracking using netted collaborative autonomous trackers. In CVPR, pages 939-946.

Zhang, J., Zhou, K., Velho, L., Guo, B., and Shum, H.-Y. [2003]. Synthesis of progressively-variant textures on arbitrary surfaces. In ACM SIGGRAPH, pages 295302.

Zhu, S., Wu, Y., and Mumford, D. [1998]. Filters random fields and maximum entropy (frame): To a unified theory for texture modeling. International Journal of Computer 
BIBLIOGRAPHY

Vision, 27(2), 107-126.

Zhu, S. C., Guo, C., Wang, Y., and Xu, Z. [2005]. What are textons? International Journal of Computer Vision, 62(1-2), 121-143.

Zucker, S. W. [1976]. Toward a model of texture. Computer Graphics and Image Processing, $5,190-202$. 


\section{APPENDIX A}

\section{Piecewise-affine Alignment of Textons}

\section{A.1. Review of Lucas-Kanade Algorithm}

Since we develop our piecewise affine image alignment algorithm based on the Lucas-Kanade algorithm [Lucas and Kanade, 1981], we briefly review the algorithm here. An excellent review of the Lucas-Kanade algorithm can be found in [Baker and Matthews, 2004]. Let $I(\mathbf{p})$ be an input image and $T(\mathbf{p})$ a template image where $\mathbf{p}=$ $(x, y)^{T}$ is the pixel coordinates. $\mathbf{W}(\mathbf{p} ; \mathbf{a})$ denotes a warp that map the pixel $\mathbf{x}$ in the coordinate frame of the template $T$ to the subpixel location $\mathbf{W}(\mathbf{p} ; \mathbf{a})$ in the coordinate frame of the image. $\mathbf{a}=\left(a_{1}, a_{2}, \ldots, a_{n}\right)^{T}$ is a vector of the warp parameters. The Lucas-Kanade algorithm aligns a template image to an input image by minimizing the following error function:

$$
\sum_{\mathbf{p}}\left[T(\mathbf{p})-I(\mathbf{W}(\mathbf{p} ; \mathbf{a})]^{2}\right.
$$

Instead of solving the nonlinear optimization directly, the Lucas-Kanade algorithm linearizes Equation (A.1) by performing a first order Taylor expansion on $I(\mathbf{W}(\mathbf{p} ; \mathbf{a}+\triangle \mathbf{a})$ to give:

$$
\sum_{\mathbf{p}}\left[T(\mathbf{p})-I(\mathbf{W}(\mathbf{p} ; \mathbf{a}))-\nabla I \frac{\partial \mathbf{W}}{\partial \mathbf{a}} \triangle \mathbf{a}\right]^{2}
$$

and incrementally minimizes the error by iteratively updating the parameters:

$$
\mathbf{a} \leftarrow \mathbf{a}+\triangle \mathbf{a}
$$

$\triangle \mathbf{p}$ is obtained by taking the partial derivative of Equation (A.2) with respect to $\triangle \mathbf{p}$, and equating the partial derivative to zero.

$$
\triangle \mathbf{a}=\mathbf{H}^{-1} \sum_{\mathbf{p}}\left[\nabla I \frac{\partial \mathbf{W}}{\partial \mathbf{a}}\right]^{T}[T(\mathbf{p})-I(\mathbf{W}(\mathbf{p} ; \mathbf{a}))]
$$


where $\mathbf{H}$ is the $n \times n$ Hessian matrix:

$$
\mathbf{H}=\sum_{\mathbf{p}}\left[\nabla I \frac{\partial \mathbf{W}}{\partial \mathbf{a}}\right]^{T}\left[\nabla I \frac{\partial \mathbf{W}}{\partial \mathbf{a}}\right]
$$

\section{A.2. Piecewise Affine Alignment for Tightly Coupled Textons}

For simplicity, we skip the time index of all variables in the following derivations. Let $\mathbf{C}=\left(c^{1 x}, c^{1 y}, c^{2 x}, c^{2 y}, \ldots, c^{m x}, c^{m y}, \ldots, c^{M x}, c^{M y}\right)$ be a vector formed by collecting the coordinates of all texton vertexes. For each quadrilateral texton, we divide it into two triangles and the vertices of each triangle are used to parameterize an affine transformation. The goal of the piecewise affine alignment is to find an optimal $\widetilde{\mathbf{C}}$ that minimizes the sum of SSD of all textons,

$$
\widetilde{\mathbf{C}}=\operatorname{argmin} \sum_{i} \sum_{\mathbf{p}} v^{i} \cdot\left[T^{i}(\mathbf{p})-I\left(\mathbf{W}\left(\mathbf{p} ; \mathbf{a}^{\mathbf{i} 1}\right)\right)\right]^{2}+\sum_{i} \sum_{\mathbf{p}} v^{i} \cdot\left[T^{i}(\mathbf{p})-I\left(\mathbf{W}\left(\mathbf{p} ; \mathbf{a}^{\mathbf{i} \mathbf{2}}\right)\right)\right]^{2}
$$

where $\mathbf{a}^{i 1}=\left(c^{i 1 x}, c^{i 1 y}, c^{i 2 x}, c^{i 2 y}, c^{i 3 x}, c^{i 3 y}\right)$ and $\mathbf{a}^{i 2}=\left(c^{i 2 x}, c^{i 2 y}, c^{i 3 x}, c^{i 3 y}, c^{i 4 x} c^{i 4 y}\right)$. Because textons are tightly coupled, a texton vertex can be shared by multiple textons (at most 4). It is possible that $\mathbf{c}^{i k}=\mathbf{c}^{j l}$ when $i \neq j$. A unique index $m$ is assigned to each texton vertex. For the $j t h$ vertex of the $i t h$ texton, its index is denoted as $m=i d x(i, j)$.

The error function related to the $\mathbf{a}^{\mathbf{i} 1}$ terms in Equation (A.6) is linearized as follows:

$$
\sum_{i} \sum_{\mathbf{p}} v^{i} \cdot\left[T^{i}(\mathbf{p})-I\left(\mathbf{W}\left(\mathbf{p} ; \mathbf{a}^{\mathbf{i} 1}\right)\right)-\nabla I \frac{\partial \mathbf{W}}{\partial \mathbf{a}^{\mathbf{i} 1}} \triangle \mathbf{a}^{\mathbf{i} 2}\right]^{2}
$$

where $\mathbf{W}\left(\mathbf{p} ; \mathbf{a}^{\mathbf{i} 1}\right)=\left[W_{x}\left(\mathbf{p} ; \mathbf{a}^{\mathbf{i} 1}\right), W_{y}\left(\mathbf{p} ; \mathbf{a}^{\mathbf{i} 1}\right)\right]^{T}$ is an affine warp whose partial derivative is

$$
\frac{\partial \mathbf{W}}{\partial \mathbf{a}^{\mathbf{i} 1}}=\left[\begin{array}{cccccc}
\frac{-x}{w-1}+\frac{-y}{h-1} & 0 & \frac{x-1}{w-1} & 0 & \frac{y-1}{h-1} & 0 \\
0 & \frac{-x}{w-1}+\frac{-y}{h-1} & 0 & \frac{x-1}{w-1} & 0 & \frac{y-1}{h-1}
\end{array}\right]
$$

Since we want to compute the partial derivative of (A.7) with respect to the position adjustments of all texton vertexes,

$$
\triangle \mathbf{C}=\left[\delta c_{1 x}, \delta c_{1 y}, \ldots, \delta c_{m x}, \delta c_{m y}, \ldots, \delta c_{M x}, \delta c_{M y}\right]^{T}
$$

we represent $\triangle \mathbf{a}^{\mathrm{i} 1}$ by

$$
\triangle \mathbf{a}^{\mathrm{i} 1}=\mathbf{B}^{\mathrm{i} 1} \triangle \mathbf{C}
$$

where

$$
B^{i 1}(l, m)= \begin{cases}1 & \text { if } l=2 l^{\prime}-1 \text { and } m=2 \cdot i d x\left(i, l^{\prime}\right)-1, l^{\prime}=1,2,3 \\ 1 & \text { if } l=2 l^{\prime} \text { and } m=2 \cdot i d x\left(i, l^{\prime}\right), l^{\prime}=1,2,3 \\ 0 & \text { otherwise }\end{cases}
$$


is a $6 \times 2 M$ matrix that picks the coordinates of the vertexes for the associated affine transformations of the texton $i$. Inserting Equation (A.10) into (A.7) and taking the partial derivative, we obtain

$$
2 \sum_{i} \sum_{\mathbf{p}} v^{i}\left[\nabla I \frac{\partial \mathbf{W}}{\partial \mathbf{a}^{\mathbf{i} 1}} \mathbf{B}^{\mathbf{i} \mathbf{1}}\right]^{T}\left[T^{i}(\mathbf{p})-I\left(\mathbf{W}\left(\mathbf{p} ; \mathbf{a}^{\mathbf{i} 1}\right)\right)-\nabla I \frac{\partial \mathbf{W}}{\partial \mathbf{a}^{\mathbf{i} 1}} \mathbf{B}^{\mathbf{i} \mathbf{1}} \triangle \mathbf{C}\right],
$$

which can be simplified to

$$
2\left(\sum_{i} v^{i} \mathbf{E}^{\mathbf{i} \mathbf{1}}-\sum_{i} v^{i} \mathbf{H}^{\mathbf{i} 1} \triangle \mathbf{C}\right)
$$

where

$$
\begin{gathered}
\mathbf{E}^{\mathbf{i} \mathbf{1}}=\sum_{\mathbf{p}}\left[\nabla I \frac{\partial \mathbf{W}}{\partial \mathbf{a}^{\mathbf{i}}} \mathbf{B}^{\mathbf{i} \mathbf{1}}\right]^{T}\left[T^{i}(\mathbf{p})-I\left(\mathbf{W}\left(\mathbf{p} ; \mathbf{a}^{\mathbf{i} \mathbf{1}}\right)\right)\right] \\
\mathbf{H}^{\mathbf{i} \mathbf{1}}=\sum_{\mathbf{p}}\left[\nabla I \frac{\partial \mathbf{W}}{\partial \mathbf{a}^{\mathbf{i} 1}} \mathbf{B}^{\mathbf{i} \mathbf{1}}\right]^{T} \nabla I \frac{\partial \mathbf{W}}{\partial \mathbf{a}^{\mathbf{i} 1}} \mathbf{B}^{\mathbf{i} \mathbf{1}}
\end{gathered}
$$

Similar to the derivation of Equation (A.13), we can obtain the partial derivative related to the $\mathbf{a}^{\mathbf{i} 2}$ terms:

$$
2\left(\sum_{i} v^{i} \mathbf{E}^{\mathbf{i} 2}-\sum_{i} v^{i} \mathbf{H}^{\mathbf{i} 2} \triangle \mathbf{C}\right)
$$

by replacing Equation (A.8) with

$$
\frac{\partial \mathbf{W}}{\partial \mathbf{a}^{\mathbf{i} 2}}=\left[\begin{array}{cccccc}
\frac{-y+h}{h-1} & 0 & \frac{-x+w}{w-1} & 0 & \frac{x}{w-1}+\frac{y}{h-1}+\frac{1-h w}{(h-1)(w-1)} & 0 \\
0 & \frac{-y+h}{h-1} & 0 & \frac{-x+w}{w-1} & 0 & \frac{x}{w-1}+\frac{y}{h-1}+\frac{1-h w}{(h-1)(w-1)}
\end{array}\right]
$$

and Equation (A.11) with

$$
B^{i 2}(l, m)= \begin{cases}1 & \text { if } l=2 l^{\prime}-1 \text { and } m=2 \cdot i d x\left(i, l^{\prime}+1\right)-1, l^{\prime}=1,2,3 \\ 1 & \text { if } l=2 l^{\prime} \text { and } m=2 \cdot i d x\left(i, l^{\prime}+1\right), l^{\prime}=1,2,3 \\ 0 & \text { otherwise }\end{cases}
$$

Finally, summing Equations (A.13) and (A.16) and equating it to zero, we can obtain the parameter updating equation:

$$
\mathbf{C}=\mathbf{C}+\left[\sum_{i} v^{i}\left(\mathbf{H}^{\mathbf{i} 1}+\mathbf{H}^{\mathbf{i} \mathbf{2}}\right)\right]^{-1} \sum_{i} v^{i}\left(\mathbf{E}^{\mathbf{i} 1}+\mathbf{E}^{\mathbf{i} \mathbf{2}}\right)
$$

\section{A.3. Affine Alignment for Loosely Coupled Textons}

For loosely coupled textons, each texton vertex only associates with one texton. Therefore, $\mathbf{a}^{i k}$ and $\mathbf{a}^{j k}$ have no shared variables when $i \neq j$. The affine alignment can be done independently for different textons, 


$$
\tilde{\mathbf{a}}^{i}=\operatorname{argmin}_{\mathbf{a}^{i}} \sum_{\mathbf{p}} v^{i} \cdot\left[T^{i}(\mathbf{p})-I\left(\mathbf{W}\left(\mathbf{p} ; \mathbf{a}^{\mathbf{i} \mathbf{1}}\right)\right)\right]^{2}+\sum_{\mathbf{p}} v^{i} \cdot\left[T^{i}(\mathbf{p})-I\left(\mathbf{W}\left(\mathbf{p} ; \mathbf{a}^{\mathbf{i} 2}\right)\right)\right]^{2} \text { (A.20) }
$$

where $\mathbf{a}^{i 1}=\left(c^{i 1 x}, c^{i 1 y}, c^{i 2 x}, c^{i 2 y}, c^{i 3 x}, c^{i 3 y}\right), \mathbf{a}^{i 2}=\left(c^{i 2 x}, c^{i 2 y}, c^{i 3 x}, c^{i 3 y}, c^{i 4 x}, c^{i 4 y}\right)$, and $\mathbf{a}^{i}=$ $\mathbf{a}^{i 1} \cup \mathbf{a}^{i 2}$. Since Equation (A.20) can be treated as a special case of Equation (A.6), the derivation is omitted here. 


\section{APPENDIX B}

\section{NRT Synthesis Comparison Results}

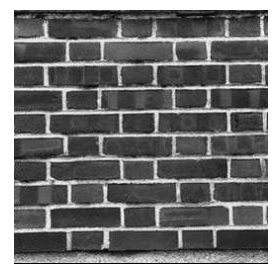

(a)input

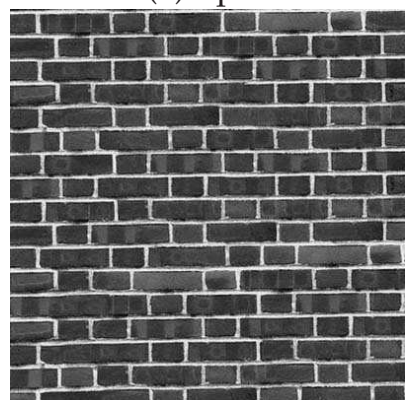

(c)pasting at local minima

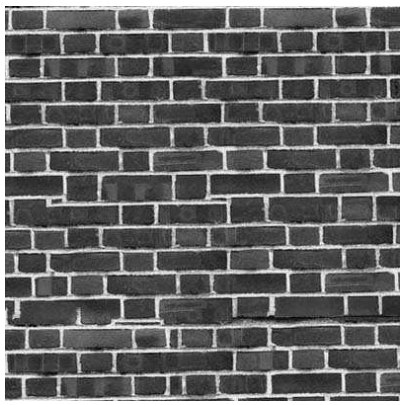

(b)pasting randomly

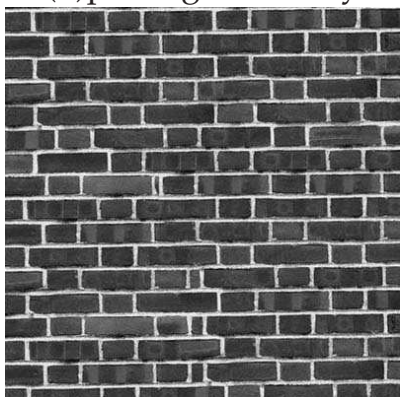

(d)pasting at local minima and maxima

Figure B.1. This figure shows the results due to different settings for patch placement in the graph cut approach. In the synthesis process, an error map is computed using seam cost around each pixel and then the location around which we want to paste the new patch is picked by sampling from this error map. The results shown are obtained respectively by (b)picking the pasting location randomly, (c)computing a local minima for these error values and picking a location from one of these, (d)computing both local minima and maxima and picking a location from one of these. The synthesis result of the same texture by the near-regular synthesis approach is shown in Figure B.12 (Texture 34).

In the following figures, those textures in the leftmost column are input textures and their ga-scores. Textures in the central and rightmost column show the synthesis results of different algorithms, where GC, NRTS, RPB, and PB corresponds to the graph cut approach, the near-regular texture synthesis approach, the regularized patch-based approach, and the patch-based approach. Those numbers in the parentheses, from left 
APPENDIX B. APPENDIX

to right, denote g-score, a-score, regularity preservation test $(\checkmark$ for preserved, $\times$ for not preserved), mean user score (best to worst: 4 to 1 ), and the standard deviation of user scores. 


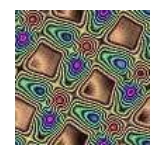

texture $1(0.00,0.00)$

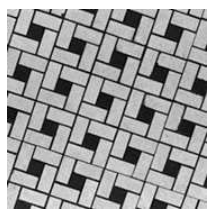

texture $2(0.00,0.00)$

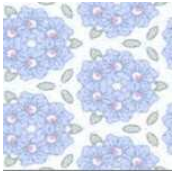

texture $3(0.00,0.00)$

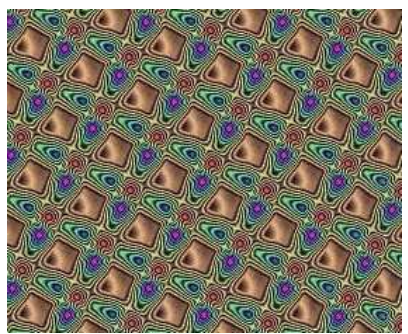

GC $(0.00,0.00, \checkmark, 3.5,0.71)$

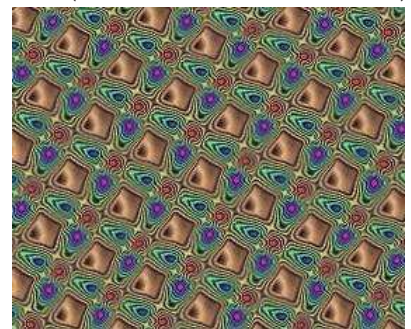

$\operatorname{RPB}(0.00,0.00, \checkmark, 3.3,0.95)$

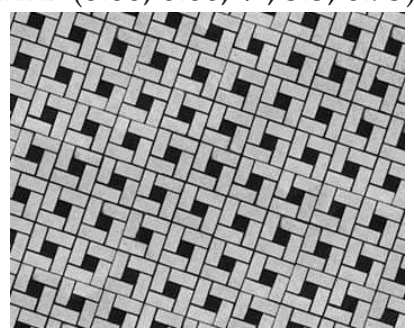

GC $(0.00,0.00, \checkmark, 3.6,0.70)$

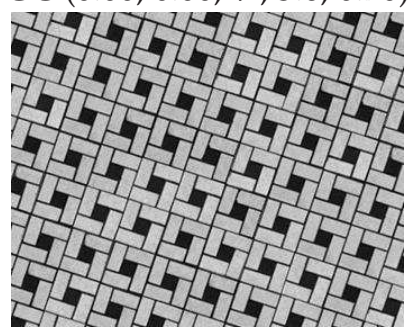

$\operatorname{RPB}(0.00,0.00, \checkmark, 3.5,0.71)$

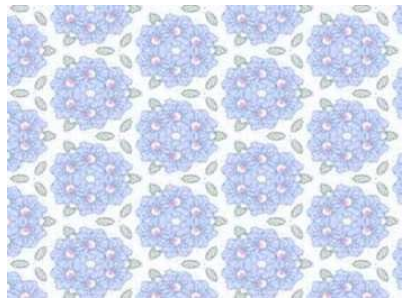

GC $(0.00,0.00, \checkmark, 3.8,0.42)$

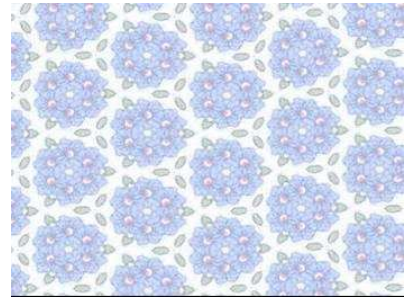

$\operatorname{RPB}(0.00,0.00, \checkmark, 3.8,0.42)$

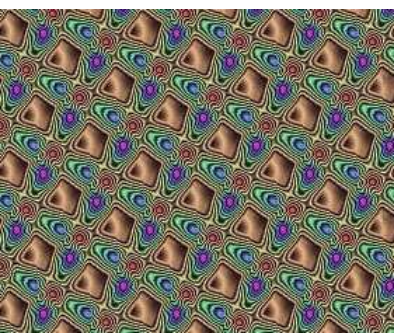

NRTS $(0.00,0.00, \checkmark, 3.6,0.70)$

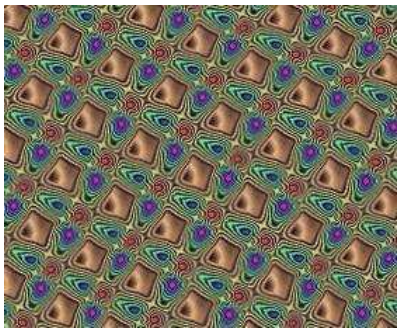

PB $(0.00,0.00, \checkmark, 3.4,0.84)$

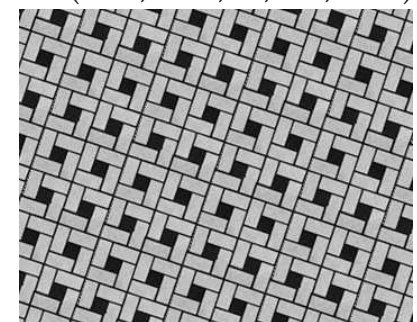

NRTS $(0.00,0.00, \checkmark, 3.6,0.84)$

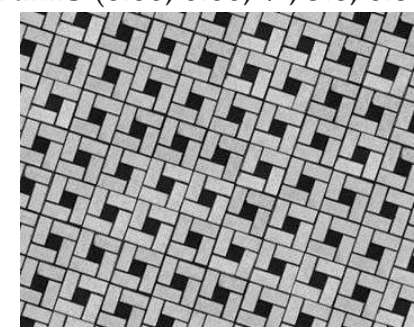

PB $(0.00,0.00, \checkmark, 3.5,0.71)$

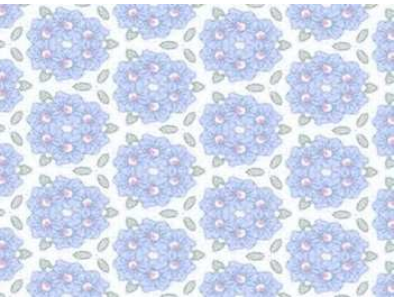

NRTS $(0.00,0.00, \checkmark, 3.8,0.42)$

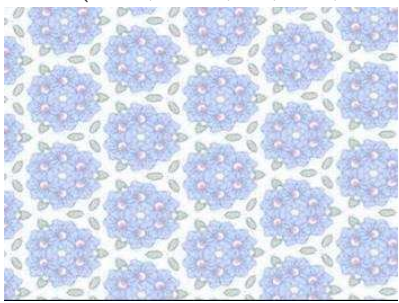

PB $(0.00,0.00, \checkmark, 3.8,0.42)$

Figure B.2 


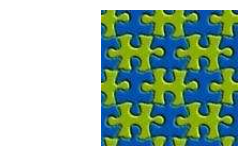

texture $4(0.00,0.06)$

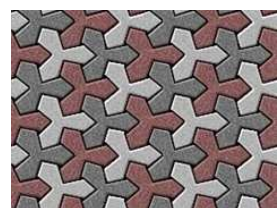

texture $5(0.00,0.10)$

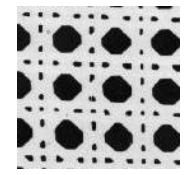

texture $6(0.46,0.11)$

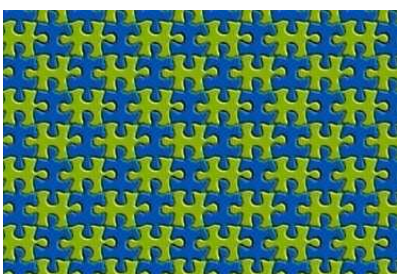

GC $(0.29,0.09, \checkmark, 3.9,0.32)$

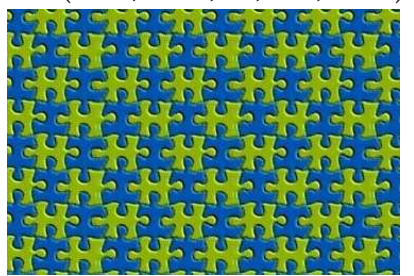

$\operatorname{RPB}(0.00,0.11, \checkmark, 3.6,0.70)$

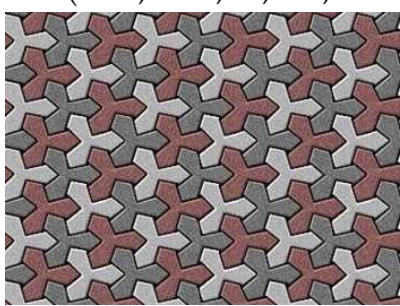

GC $(0.00,0.13, \checkmark, 3.9,0.32)$

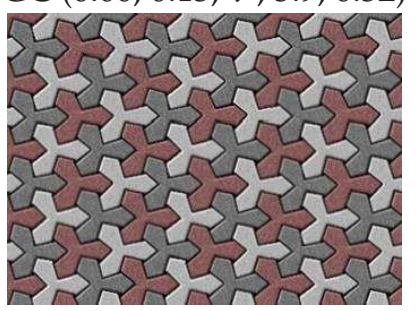

$\operatorname{RPB}(0.00,0.10, \checkmark, 3.7,0.48)$
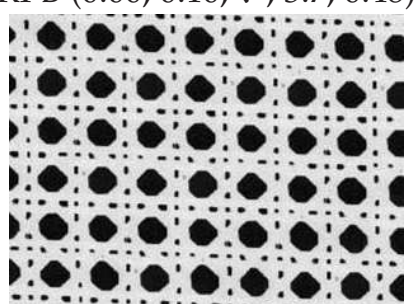

GC $(0.89,0.17, \checkmark, 3.8,0.63)$

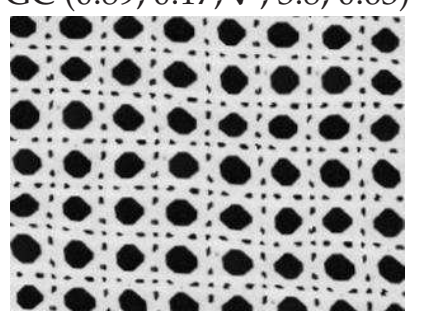

$\operatorname{RPB}(0.88,0.17, \checkmark, 2.6,1.07)$

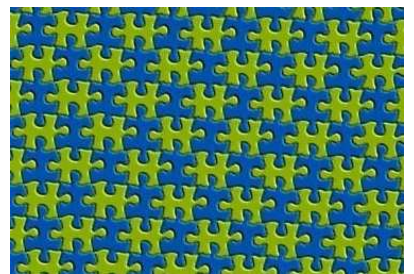

NRTS $(0.21,0.08, \checkmark, 3.8,0.42)$

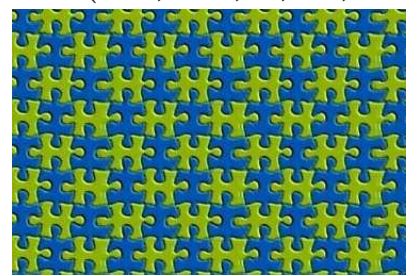

PB $(0.24,0.10, \checkmark, 3.6,0.70)$

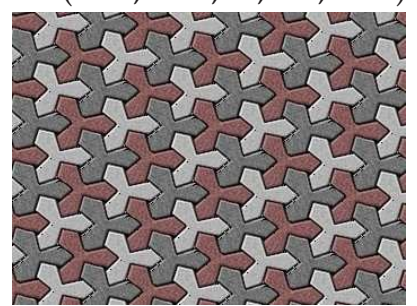

NRTS $(0.00,0.14, \checkmark, 3.6,0.52)$

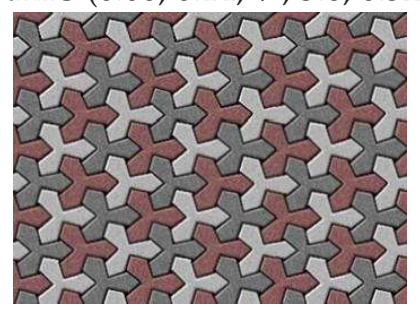

PB (0.00, 0.09, $\checkmark, 3.7,0.48)$

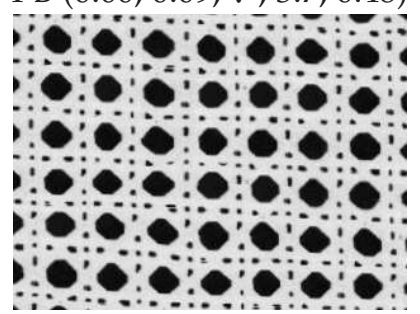

NRTS $(0.49,0.13, \checkmark, 3.1,0.88)$

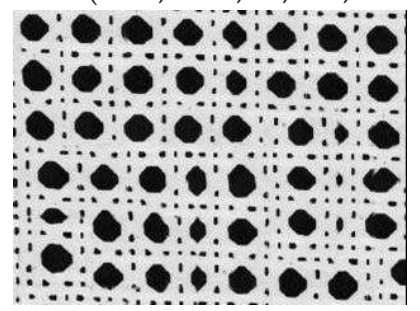

PB (1.53, 0.23, × , 1.5, 0.85)

Figure B.3 


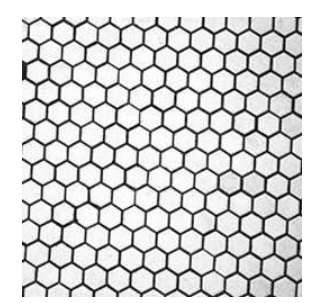

texture $7(0.00,0.16)$

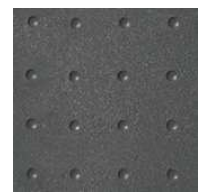

texture $8(0.00,0.03)$

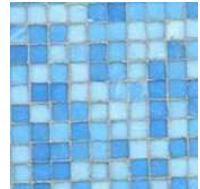

texture $9(0.17,0.09)$

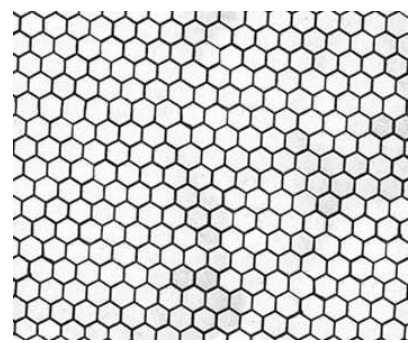

GC $(0.00,0.19, \checkmark, 3.7,0.48)$
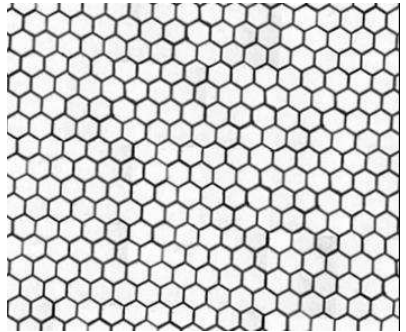

RPB $(0.00,0.26, \checkmark, 3.4,0.84)$

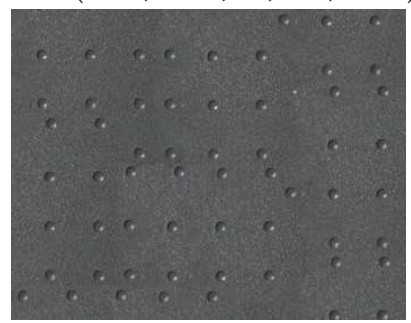

GC $(9.01,0.03, \times, 1.5,0.97)$

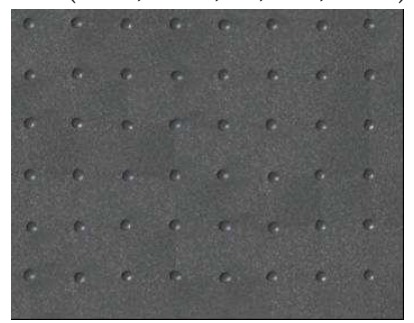

$\mathrm{RPB}(0.00,0.02, \checkmark, 3.4,0.70)$

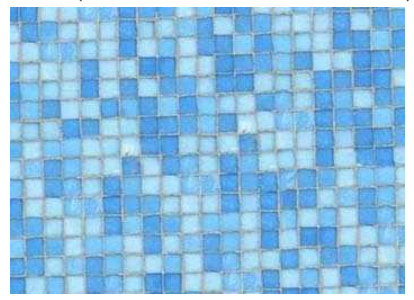

GC $(0.51,0.09, \checkmark, 3.5,0.71)$

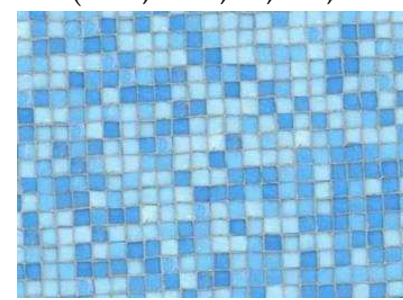

$\operatorname{RPB}(1.71,0.09, \checkmark, 2.8,0.79)$

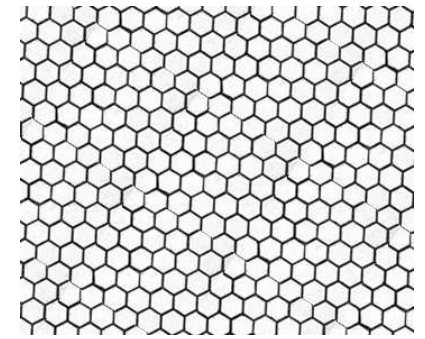

NRTS $(0.00,0.16, \checkmark, 3.6,0.70)$
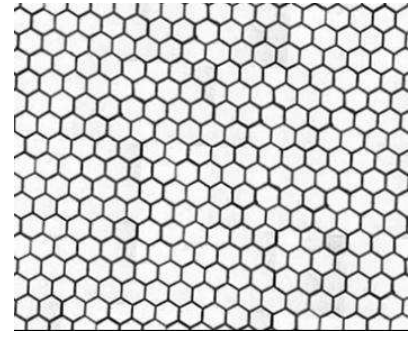

PB $(0.00,0.21, \checkmark, 3.5,0.71)$

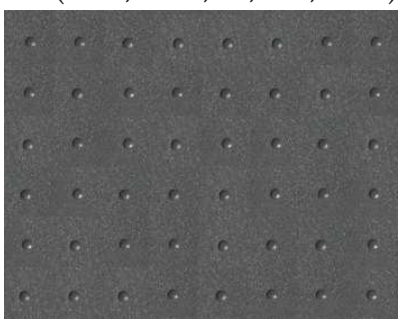

NRTS $(0.00,0.03, \checkmark, 3.3,0.82)$

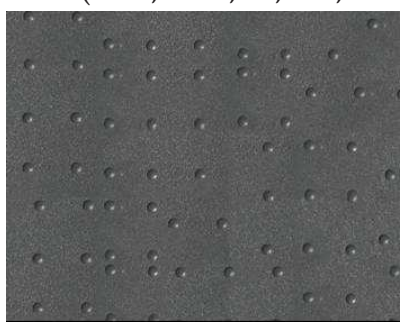

PB (10.93, 0.03, × , 1.3, 0.67)

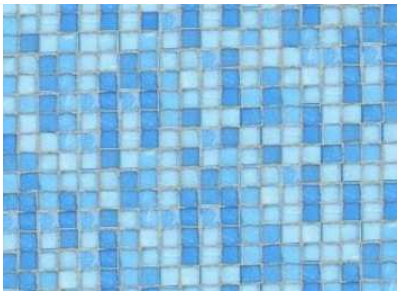

NRTS $(0.52,0.09, \checkmark, 3.1,0.88)$

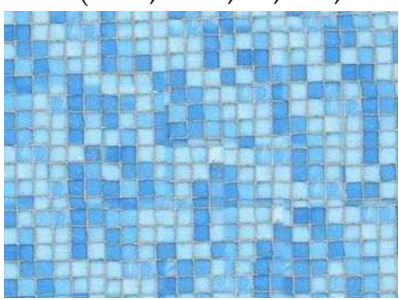

PB $(2.57,0.09, \times, 2.4,0.97)$

Figure B.4 


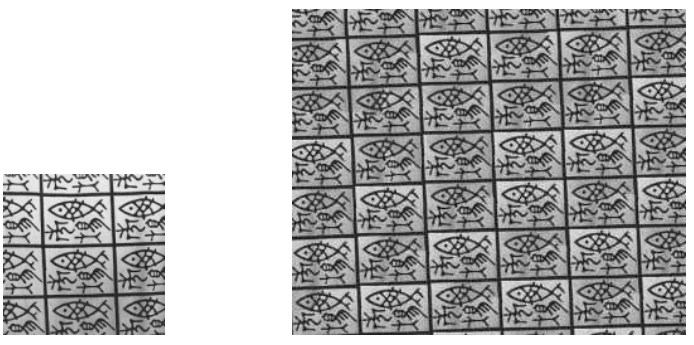

texture $10(0.07,0.11) \quad \mathrm{GC}(0.15,0.12, \checkmark, 3.7,0.48)$
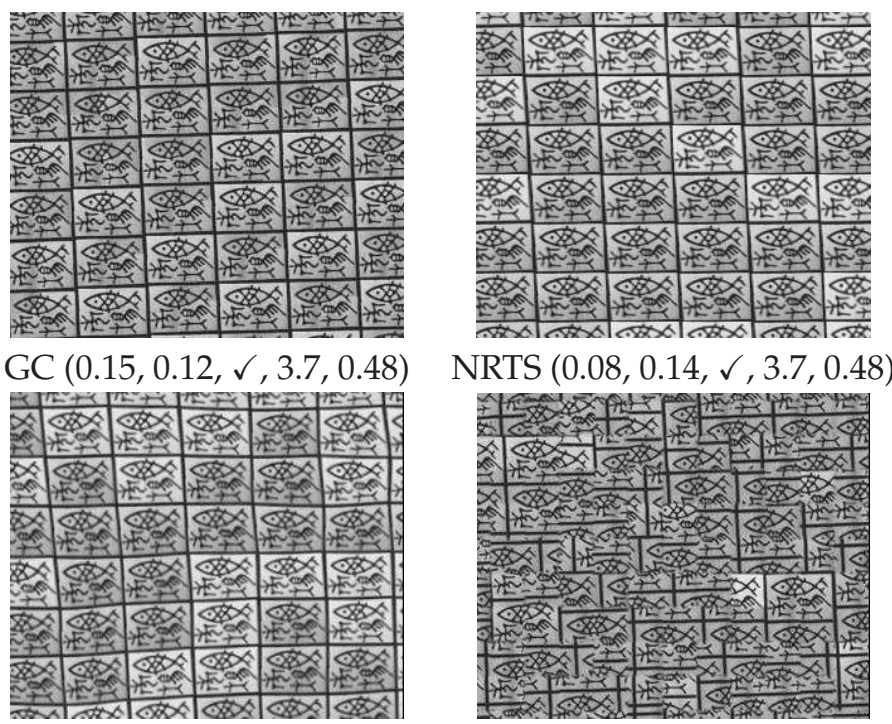

NRTS $(0.08,0.14, \checkmark, 3.7,0.48)$

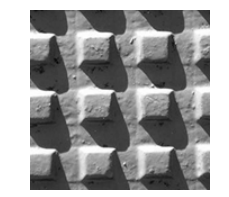

texture $11(0.00,0.04)$

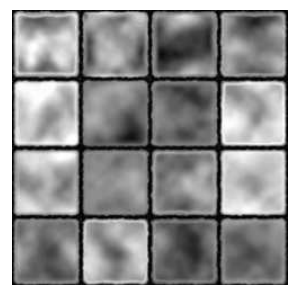

texture $12(0.24,0.15)$
RPB $(0.76,0.14, \checkmark, 2.6,0.84)$

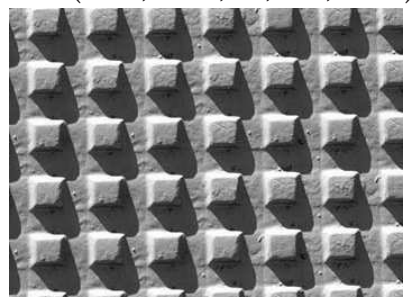

GC $(0.00,0.10, \checkmark, 3.9,0.32)$

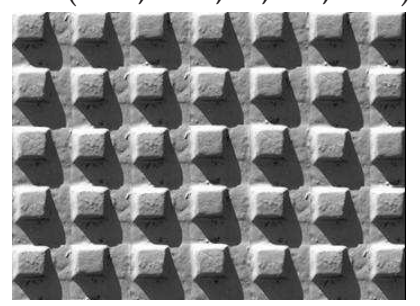

$\operatorname{RPB}(0.00,0.12, \checkmark, 3.7,0.48)$

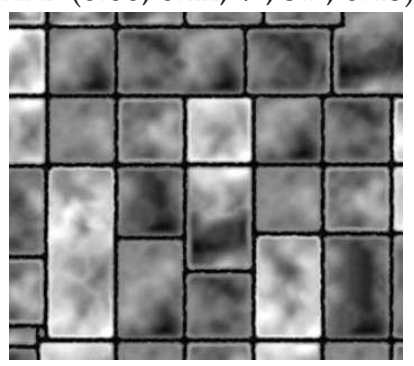

$\mathrm{GC}(6.68,0.19, \times, 1.1,0.32)$

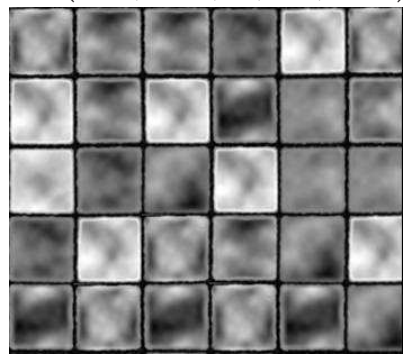

$\operatorname{RPB}(0.30,0.17, \checkmark, 3.5,0.71)$

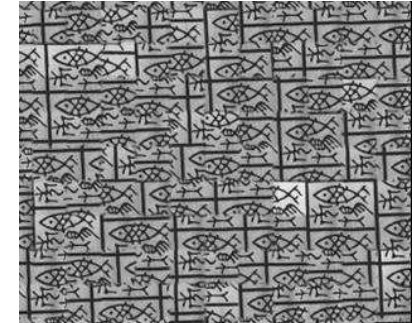

PB $(9.21,0.18, \times, 1.1,0.32)$

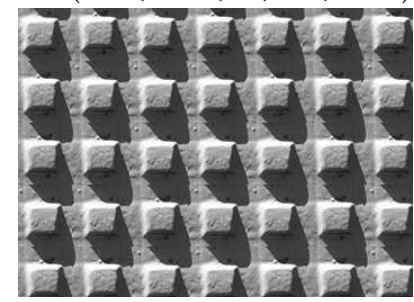

$\operatorname{NRTS}(0.00,0.10, \checkmark, 3.3,0.82)$

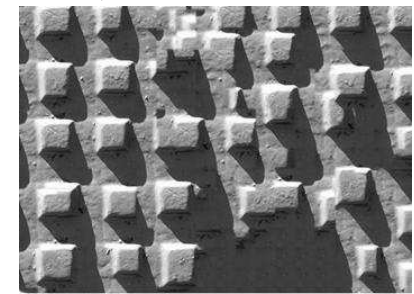

PB $(4.66,0.18, \times, 1.0,0.00)$

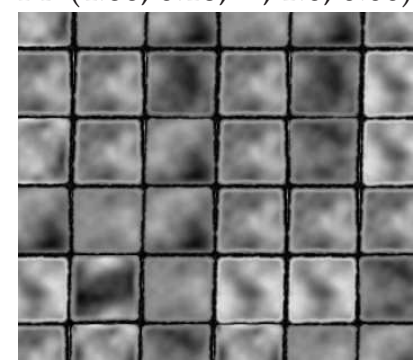

NRTS $(0.12,0.14, \checkmark, 3.4,0.52)$

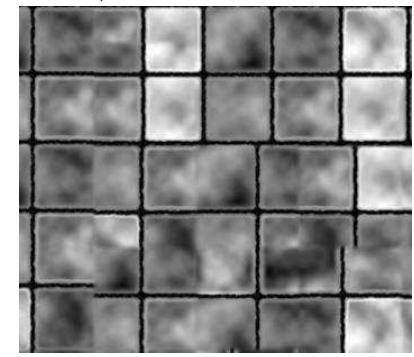

PB (11.31, 0.19, × 1.1, 0.32)

Figure B.5 


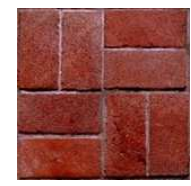

texture $13(0.00,0.00)$

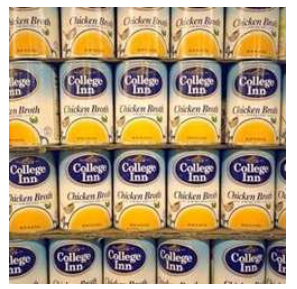

texture $14(0.00,0.14)$

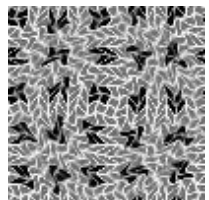

texture $15(0.37,0.17)$

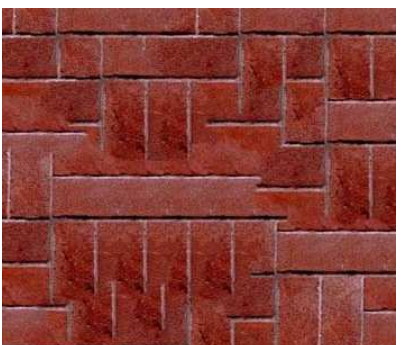

GC $(27.00,0.08, \times, 1.4,0.52)$

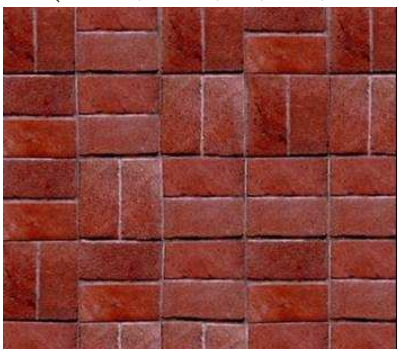

$\operatorname{RPB}(27.00,0.05, \times, 3.2,0.63)$

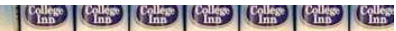

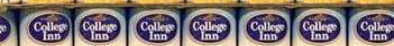
50 - $=0$

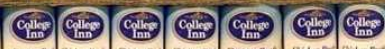

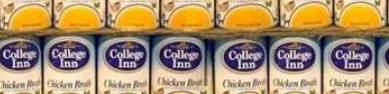

GC $(0.57,0.16, \checkmark, 3.0,0.82)$

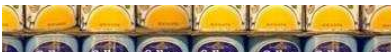

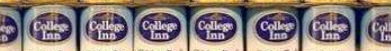
(1)

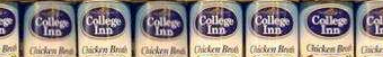
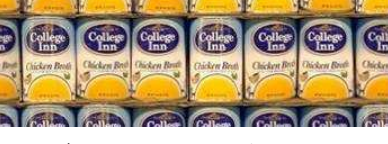

$\operatorname{RPB}(0.13,0.13, \checkmark, 3.5,0.53)$

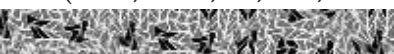

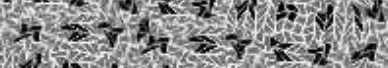

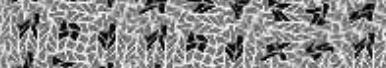
$y=2, x-2,1$

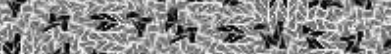

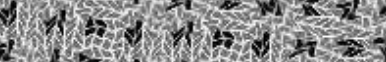

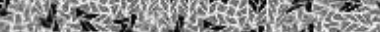
2.

GC $(1.35,0.21, \checkmark, 3.4,0.84)$

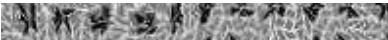

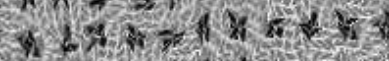

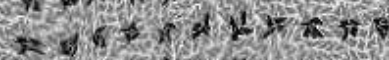

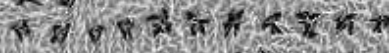

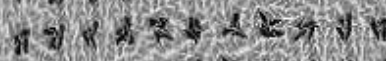

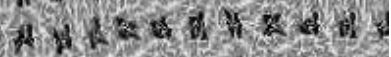

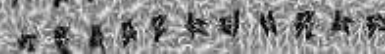

RPB $(1.64,0.14, \checkmark, 2.6,1.07)$

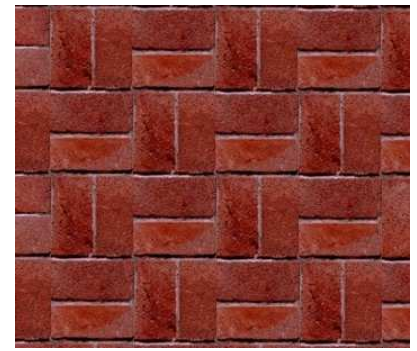

$\operatorname{NRTS}(0.00,0.08, \checkmark, 3.6,0.52)$

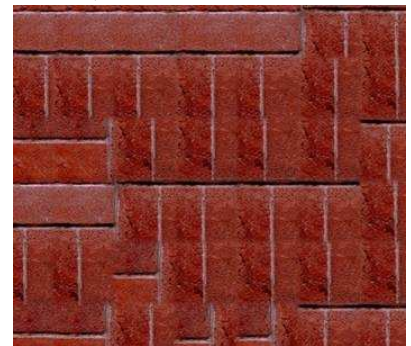

PB $(27.00,0.07, \times, 1.1,0.32)$

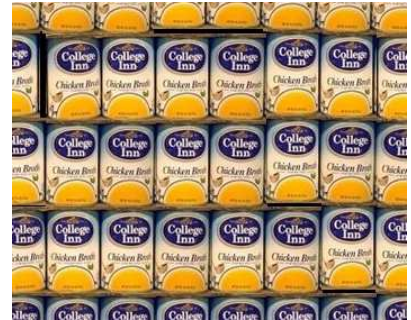

NRTS $(0.14,0.16, \checkmark, 3.0,1.05)$

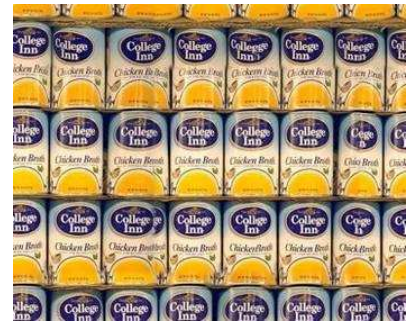

PB $(0.66,0.16, \times, 2.5,0.97)$

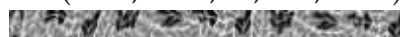

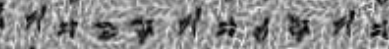
$4 x+24 x+24 x$

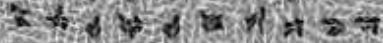
$4 x+2$ क्ष $3 x$, 1 है

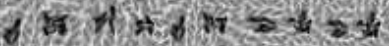

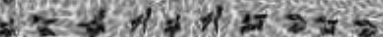

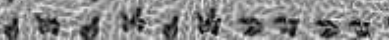

NRTS $(0.96,0.15, \checkmark, 3.4,0.84)$

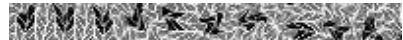

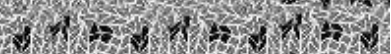
$2 x+1,7,2,4=2$

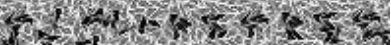
$4322,1982-2=2$

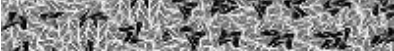

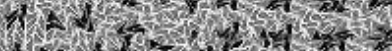

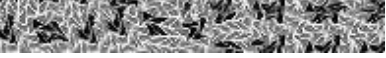
PB (2.19, 0.19, × 2.6, 1.17)

Figure B.6 


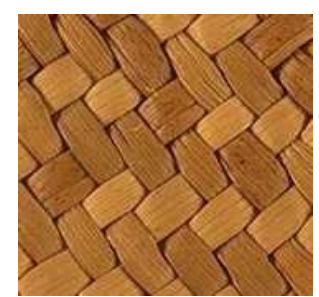

texture $16(0.25,0.07)$

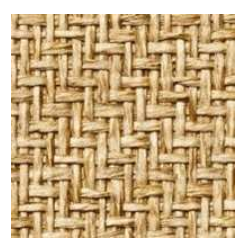

texture $17(0.00,0.13)$

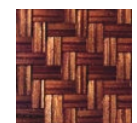

texture $18(0.00,0.09)$
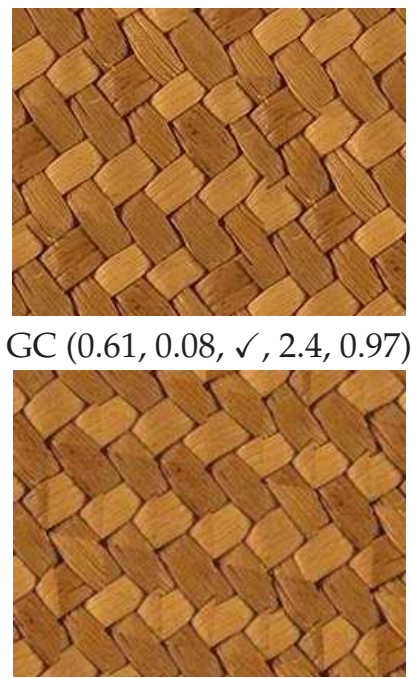

$\operatorname{RPB}(0.73,0.06, \checkmark, 2.9,0.57)$
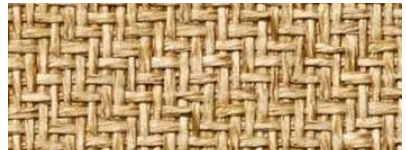

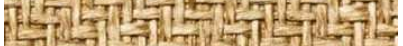

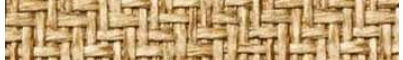

If

जा

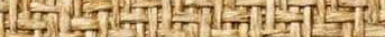

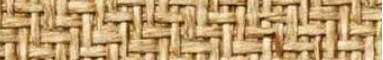

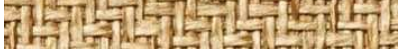

GC $(0.00,0.16, \checkmark, 3.9,0.32)$
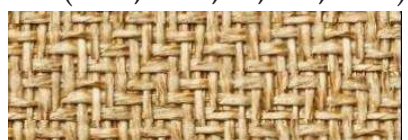

$\rightarrow \rightarrow 4$ a

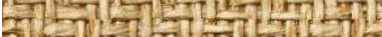

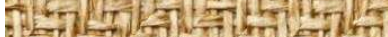

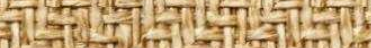

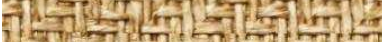
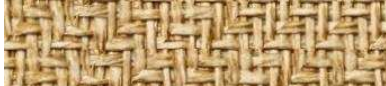

$\operatorname{RPB}(0.00,0.16, \checkmark, 3.5,0.71)$

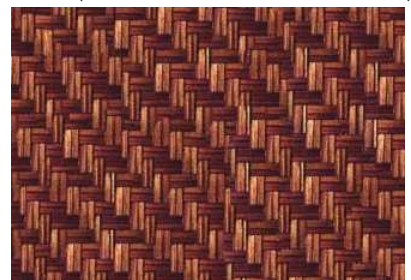

GC $(0.00,0.13, \checkmark, 3.7,0.48)$

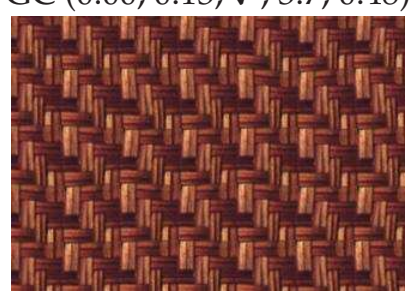

$\operatorname{RPB}(0.00,0.12, \checkmark, 2.8,0.92)$

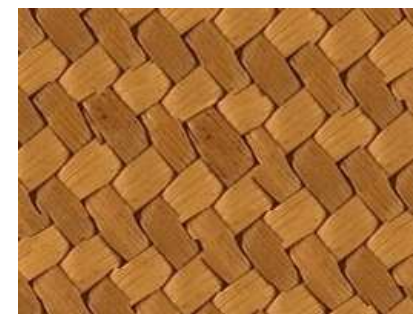

NRTS $(0.25,0.06, \checkmark, 3.3,0.82)$

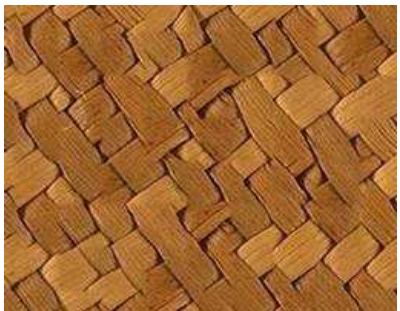

PB $(27.00,0.09, \times, 1.6,1.07)$

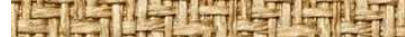

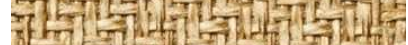

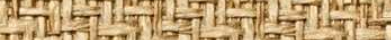

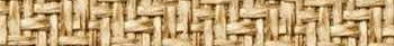

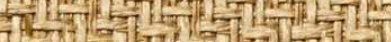

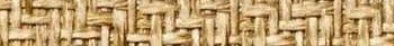

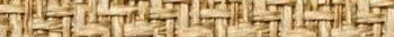

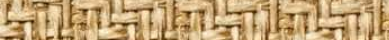

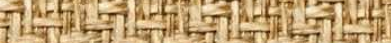

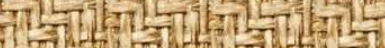

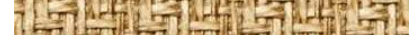

NRTS $(0.00,0.17, \checkmark, 3.7,0.48)$

7ifm

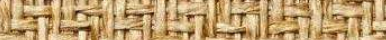

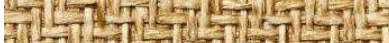

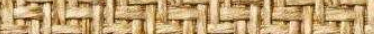

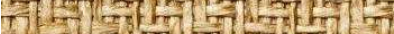

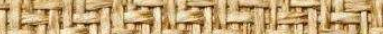

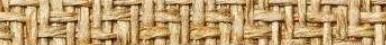

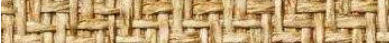

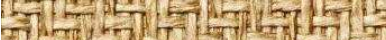

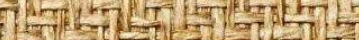

दा!

PB $(0.00,0.17, \checkmark, 3.6,0.70)$

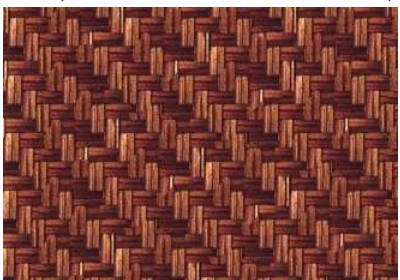

NRTS $(0.00,0.13, \checkmark, 3.4,0.84)$

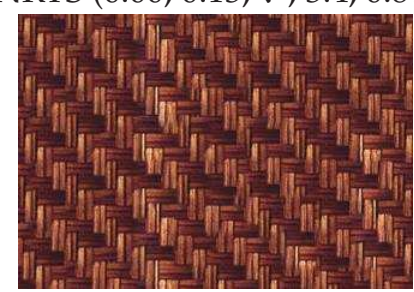

PB $(0.00,0.12, \checkmark, 2.9,0.88)$ 


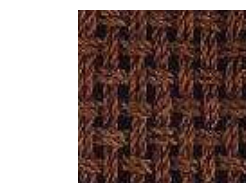

texture $19(0.00,0.08)$

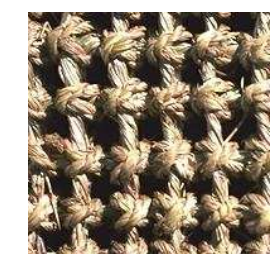

texture $20(0.40,0.17)$

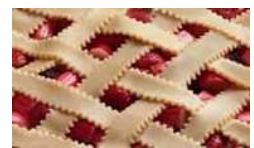

texture $21(0.39,0.00)$

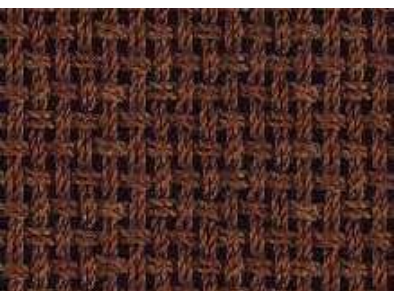

$\mathrm{GC}(0.00,0.10, \checkmark, 3.8,0.42)$

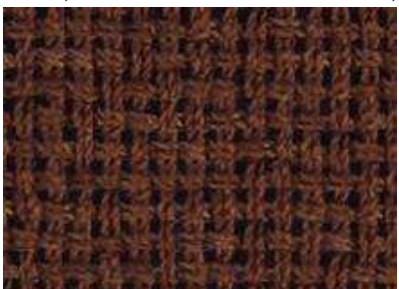

$\operatorname{RPB}(0.00,0.07, \checkmark, 3.0,0.94)$
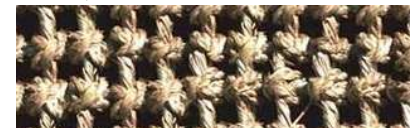

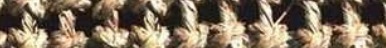

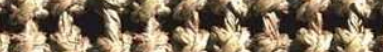

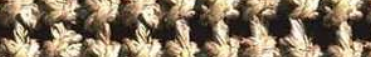

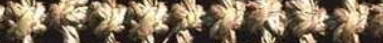

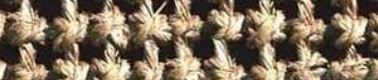

GC $(4.17,0.19, \checkmark, 3.8,0.42)$

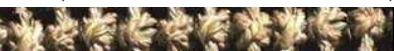

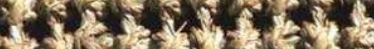

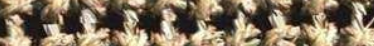

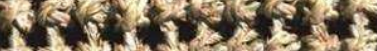

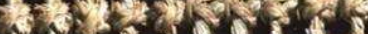

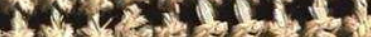

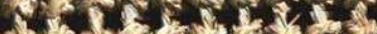
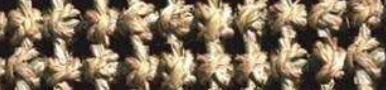

$\operatorname{RPB}(5.78,0.17, \checkmark, 3.4,0.84)$

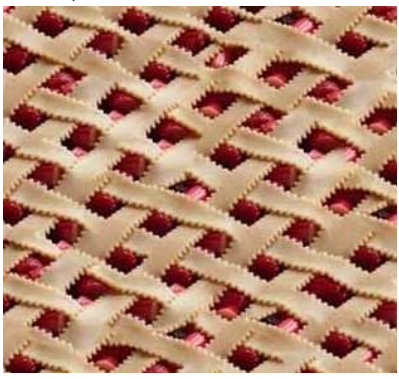

GC $(3.33,0.17, \times, 2.9,0.99)$

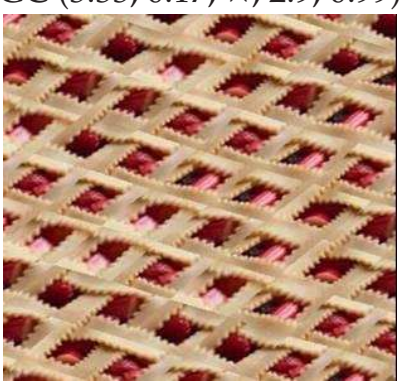

RPB $(7.06,0.13, \times, 2.3,0.48)$

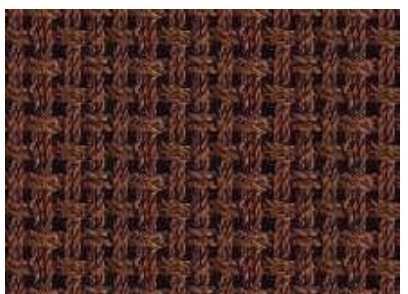

NRTS $(0.00,0.10, \checkmark, 3.4,0.97)$

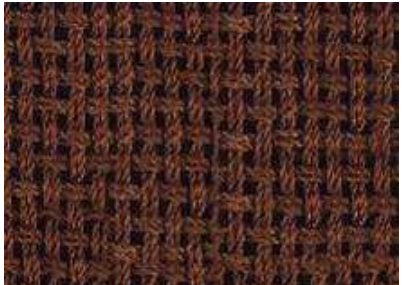

PB $(0.00,0.09, \checkmark, 2.9,1.20)$
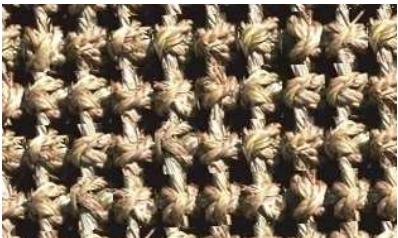

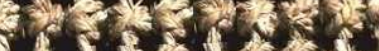

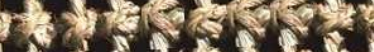

NRTS $(1.29,0.19, \checkmark, 3.5,0.71)$

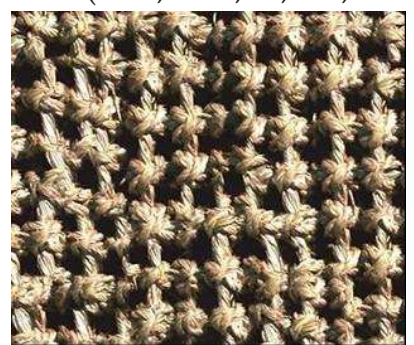

PB $(6.72,0.26, \times, 1.3,0.48)$

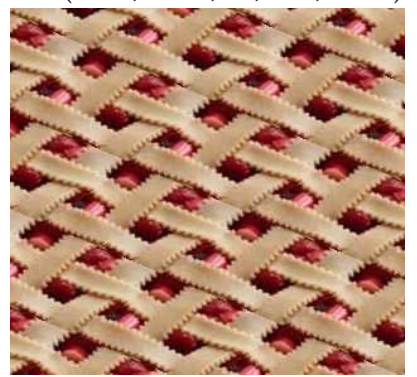

$\operatorname{NRTS}(2.66,0.07, \checkmark, 3.6,0.70)$

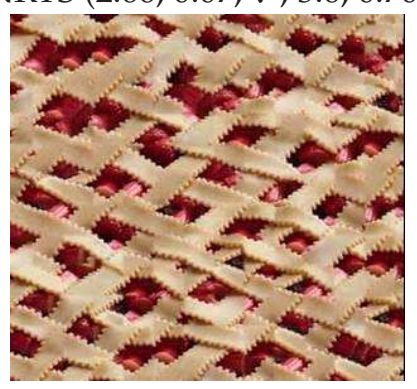

PB (27.00, 0.21, × , 1.1, 0.32)

Figure B.8 
|몰 I国回国|

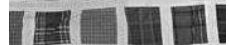

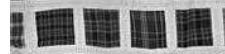

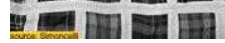

texture $22(0.50,0.09)$

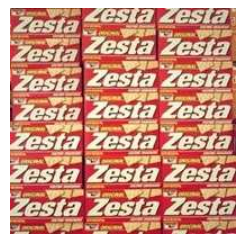

texture $23(0.00,0.17)$

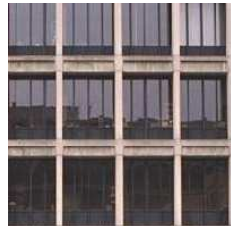

texture $24(0.00,0.05)$
F-闻的国|

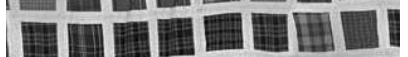

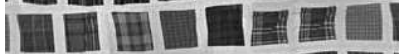

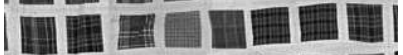

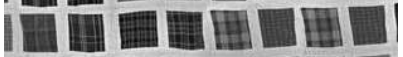

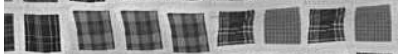

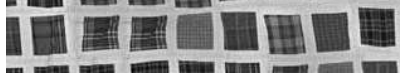

GC $(0.55,0.12, \checkmark, 3.0,0.94)$

III

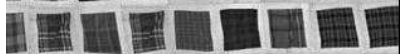

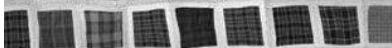

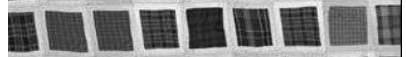

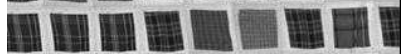

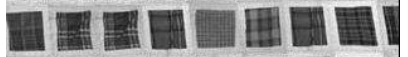

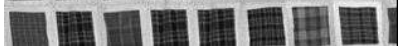

$\operatorname{RPB}(1.04,0.11, \checkmark, 3.2,0.63)$

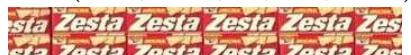

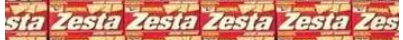

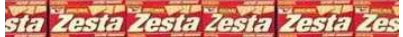

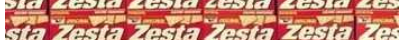

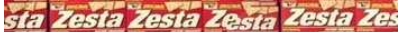

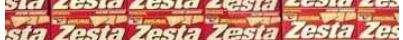

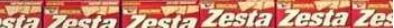

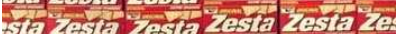

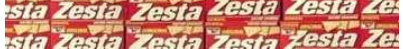
GC $(0.00,0.22, \checkmark, 3.2,0.92)$

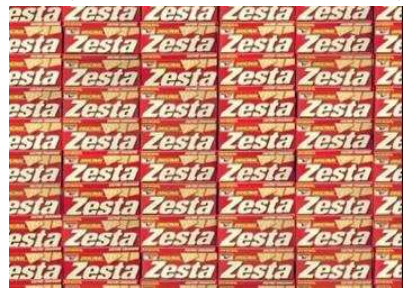

$\operatorname{RPB}(0.00,0.17, \checkmark, 3.5,0.71)$

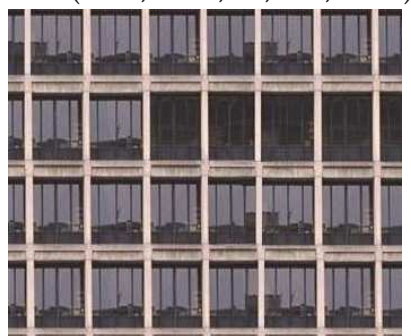

GC $(0.00,0.13, \checkmark, 3.4,0.97)$

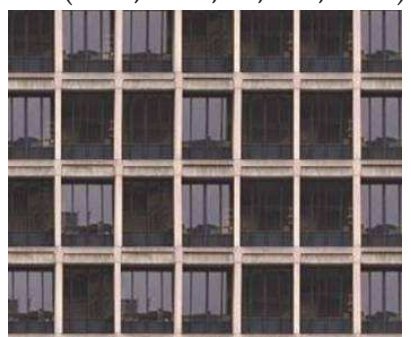

RPB $(0.00,0.08, \checkmark, 2.4,1.07)$

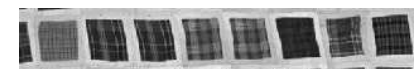

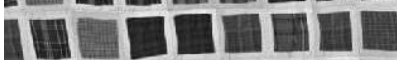

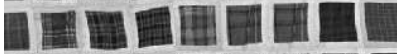

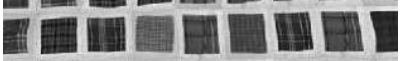

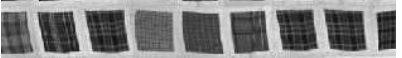

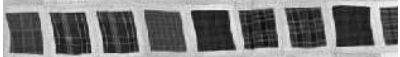

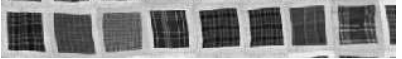

NRTS $(1.13,0.12, \checkmark, 3.7,0.48)$

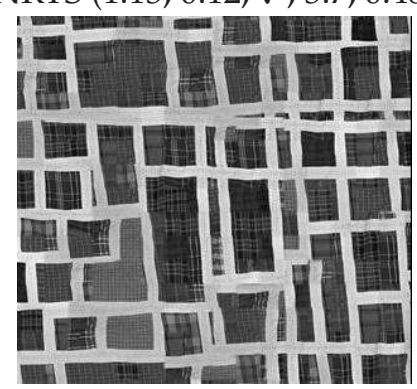

PB $(16.31,0.21, \times, 1.4,0.97)$

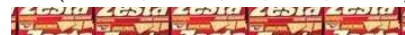

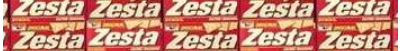

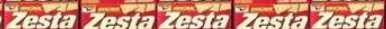

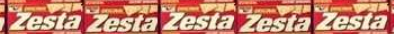

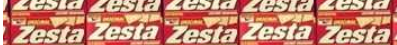

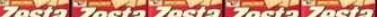
KOST ZESTA LEST ZEST LOST:

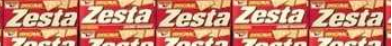

NRTS $(0.00,0.24, \checkmark, 3.8,0.42)$ zeranyary

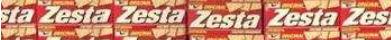

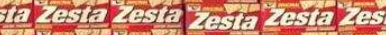

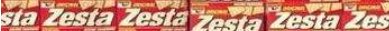

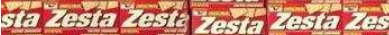

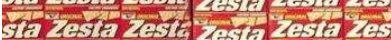

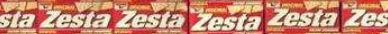

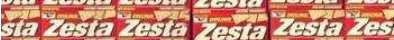

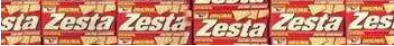

PB $(0.00,0.19, \checkmark, 2.8,1.03)$

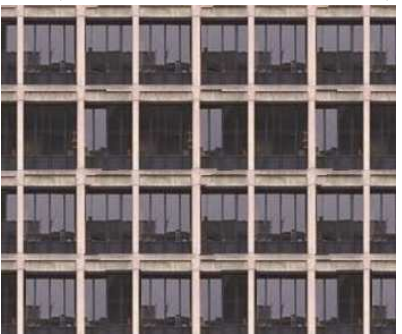

NRTS $(0.00,0.10, \checkmark, 2.6,0.97)$

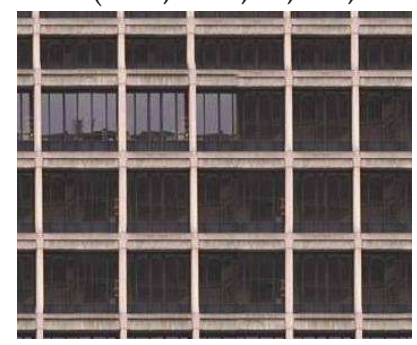

PB (15.34, 0.11, × , 1.3, 0.48)

Figure B.9 


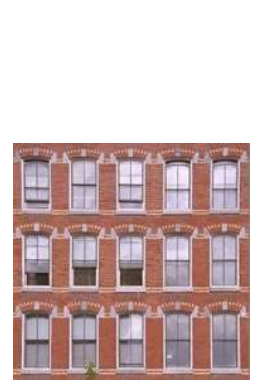

texture $25(0.00,0.08)$

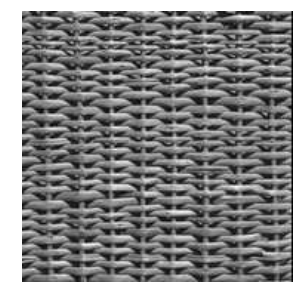

texture $26(0.33,0.16)$

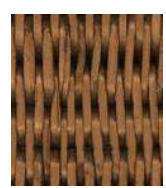

texture $27(0.07,0.06)$

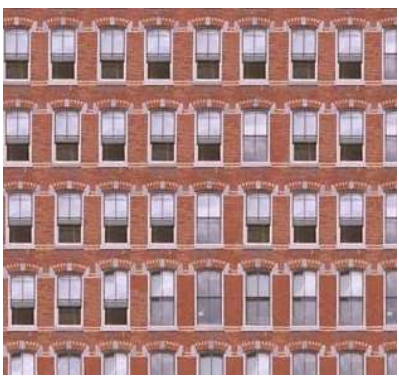

GC $(0.00,0.12, \checkmark, 3.3,0.82)$

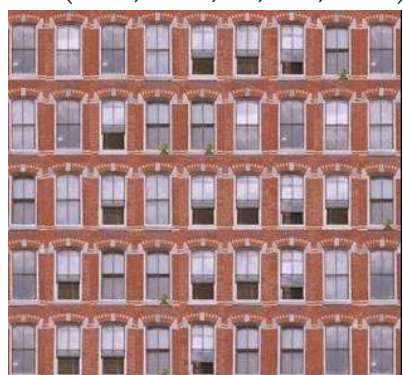

$\operatorname{RPB}(0.00,0.08, \checkmark, 3.5,0.71)$

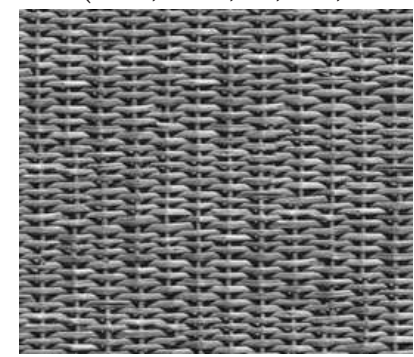

GC $(0.63,0.16, \times, 3.4,0.70)$

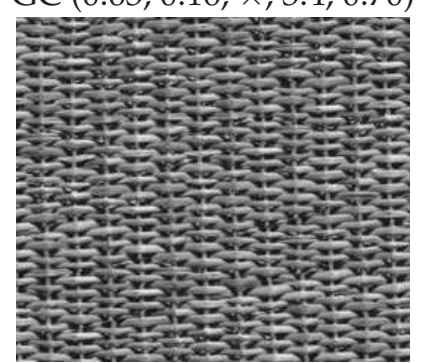

RPB $(1.01,0.14, \checkmark, 3.0,0.94)$

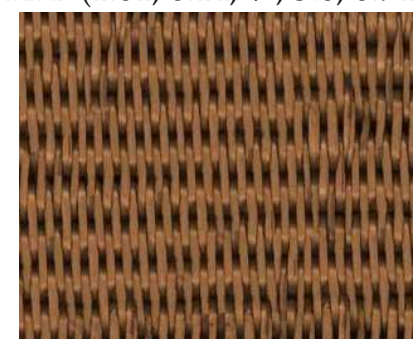

$\operatorname{GC}(0.55,0.09, \checkmark, 3.2,0.63)$

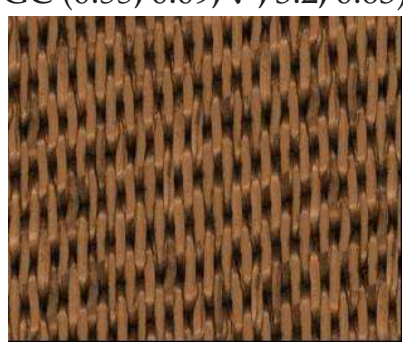

$\operatorname{RPB}(2.59,0.07, \times, 2.2,0.63)$

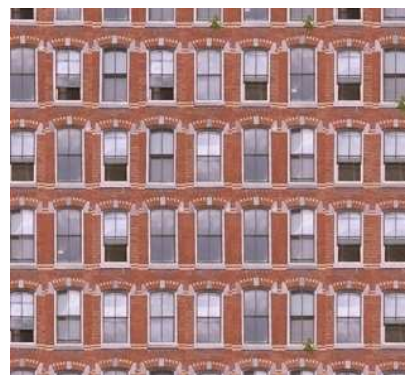

NRTS $(0.00,0.12, \checkmark, 3.7,0.48)$

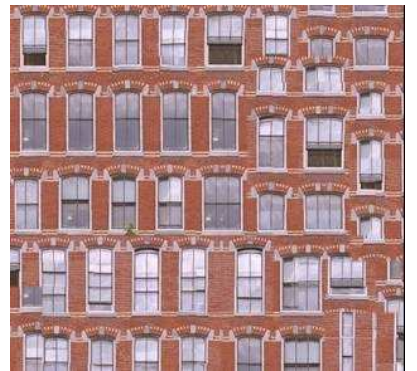

PB $(27.06,0.14, \times, 1.2,0.63)$

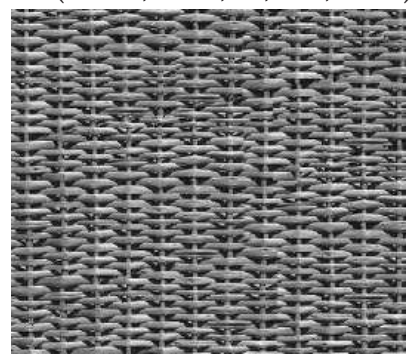

NRTS $(0.50,0.17, \checkmark, 3.2,0.79)$

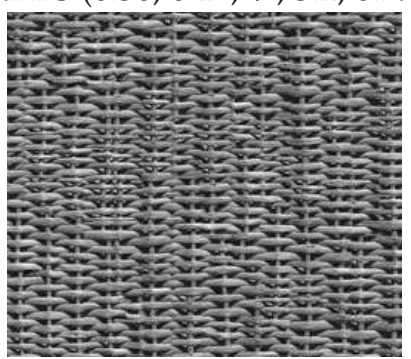

PB (3.20, 0.18, $\times, 1.4,0.52)$

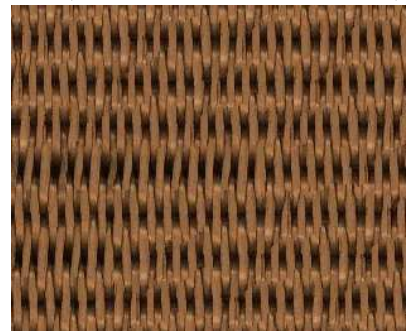

$\operatorname{NRTS}(0.36,0.08, \checkmark, 3.5,0.71)$

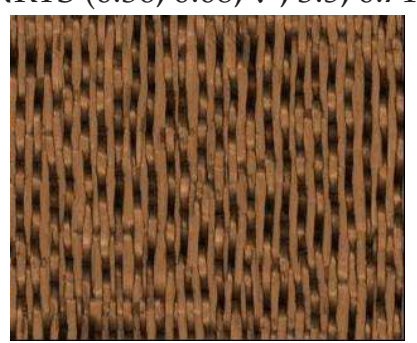

PB $(27.00,0.13, \times, 1.1,0.32)$ 

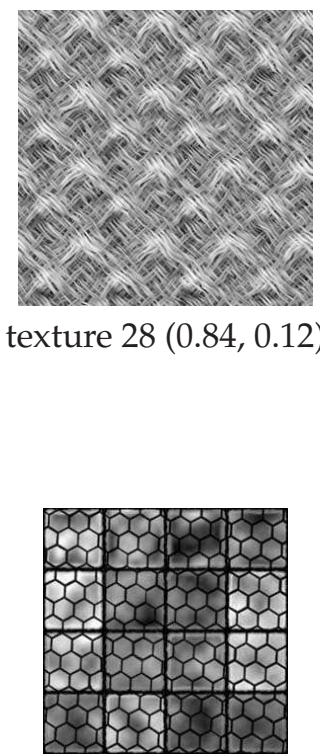

texture $29(0.00,0.17)$

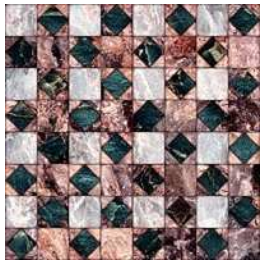

texture $30(0.18,0.14)$

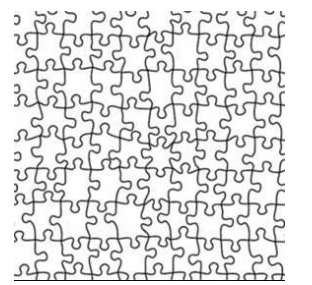

texture $31(1.76,0.14)$

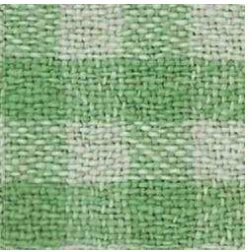

texture $32(0.32,0.08)$ texture $28(0.84,0.12)$

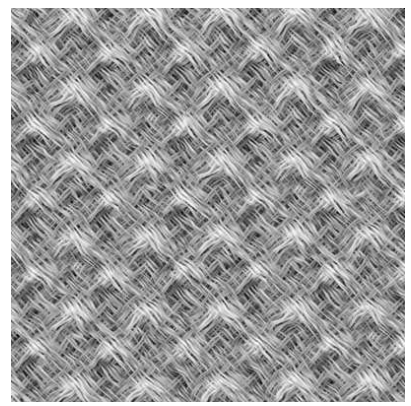

GC $(1.00,0.12, \checkmark, 3.9,0.32)$

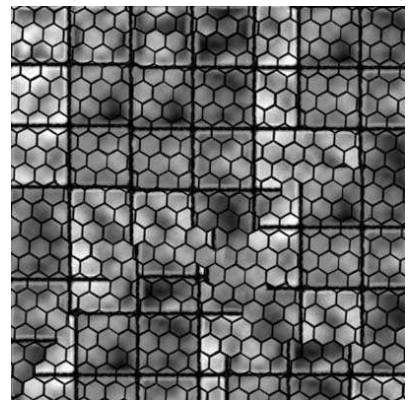

GC $(13.65,0.21, \times 1.5,0.97)$

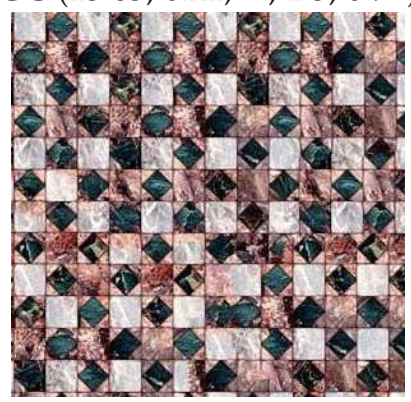

GC $(0.39,0.22, \times, 2.2,1.03)$

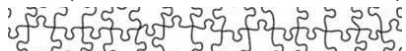

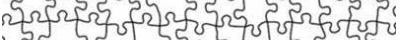

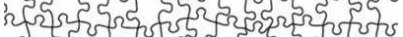

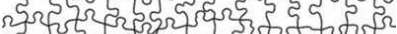

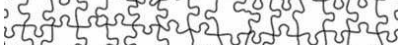

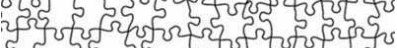

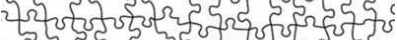

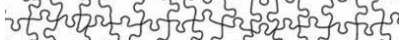

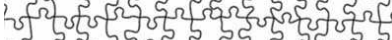

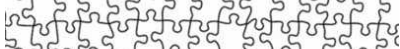

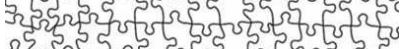
GC $(0.86,0.17, \checkmark, 3.5,0.53)$

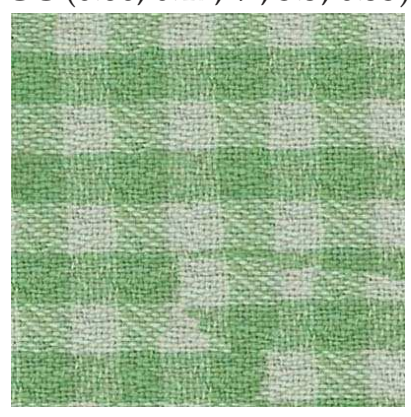

GC $(24.68,0.09, \times, 1.3,0.48)$

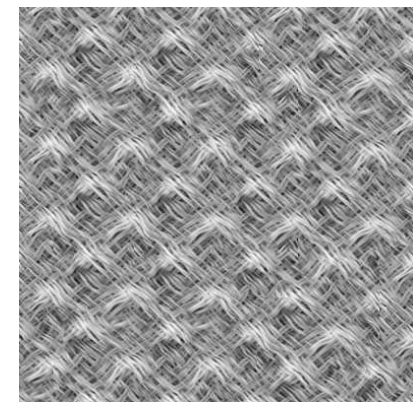

NRTS $(0.65,0.12, \checkmark, 3.6,0.52)$

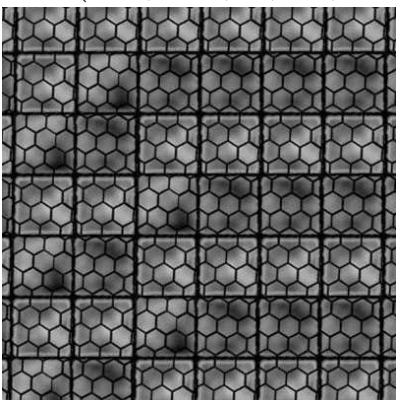

NRTS $(0.00,0.17, \times, 3.1,0.74)$

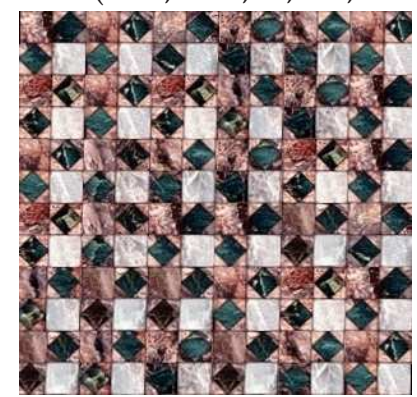

NRTS $(0.08,0.15, \checkmark, 3.6,0.70)$

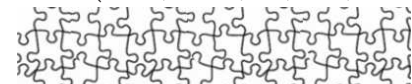

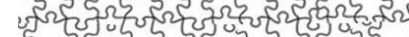

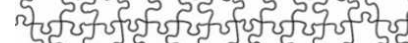

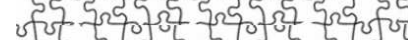
c5r-

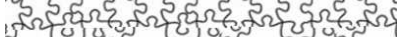
पर

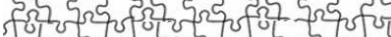
5r

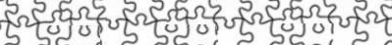

NRTS $(0.00,0.13, \times, 2.4,0.97)$

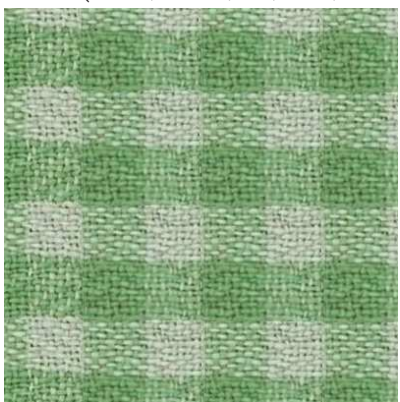

NRTS $(0.13,0.06, \checkmark, 3.8,0.42)$

Figure B.11 


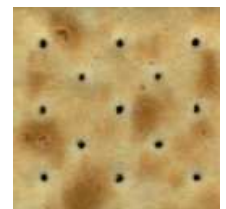

texture $33(0.14,0.06)$

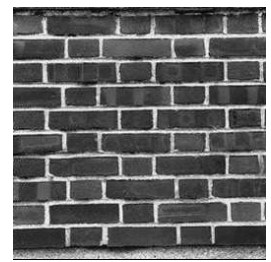

texture $34(0.20,0.12)$

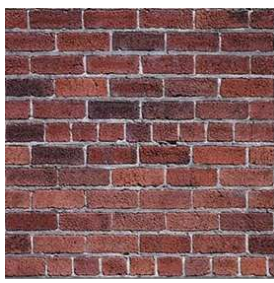

texture $35(0.00,0.10)$

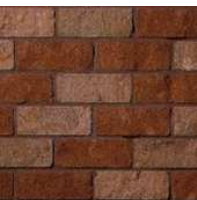

texture $36(0.58,0.07)$

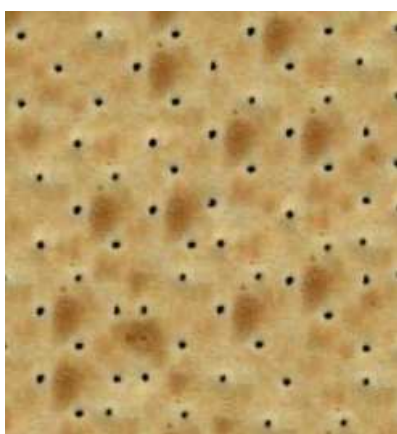

GC $(4.46,0.07, \times, 2.2,0.92)$

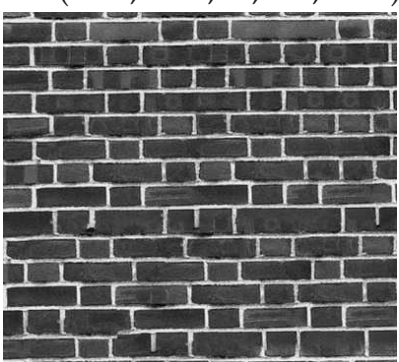

GC $(0.94,0.15, \times, 2.6,1.17)$

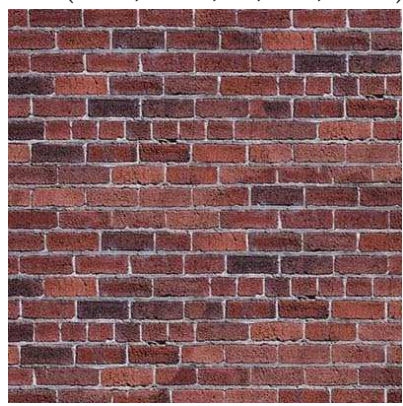

$\operatorname{GC}(0.57,0.10, \times, 3.4,0.70)$

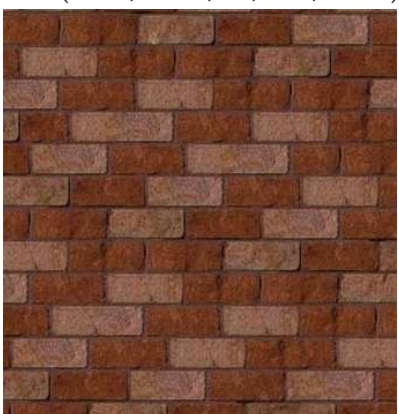

GC $(2.90,0.08, \times, 2.9,0.57)$

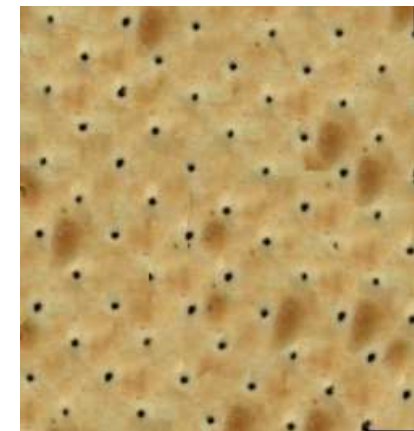

NRTS $(0.56,0.05, \checkmark, 3.7,0.48)$

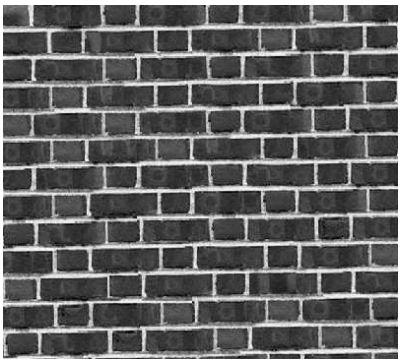

NRTS $(0.40,0.15, \checkmark, 3.8,0.42)$

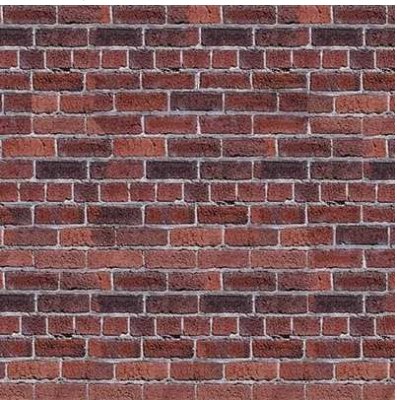

NRTS $(0.04,0.11, \checkmark, 3.4,0.70)$

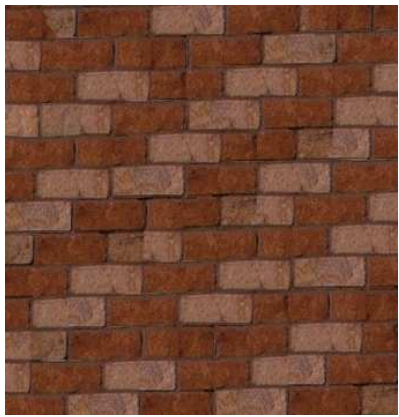

NRTS $(0.31,0.07, \checkmark, 3.3,0.48)$

Figure B.12 


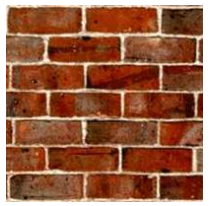

texture $37(0.20,0.12)$

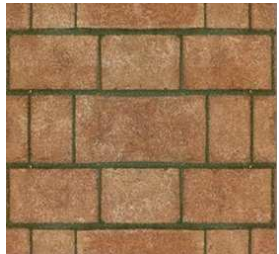

texture $38(0.00,0.00)$

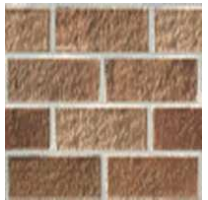

texture $39(0.01,0.09)$

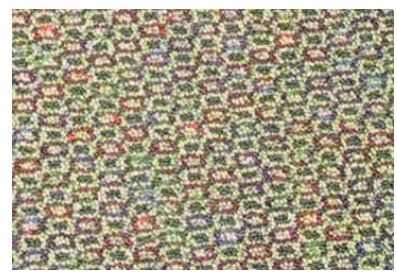

texture $40(0.00,0.18)$
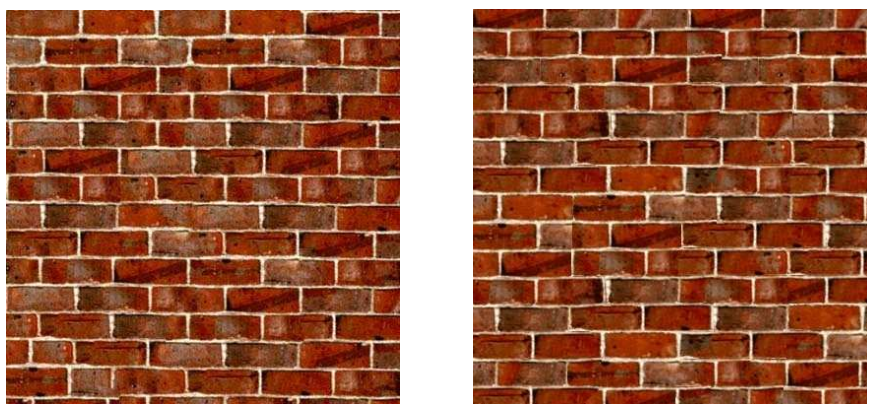

GC $(0.30,0.13, \checkmark, 3.2,0.79)$

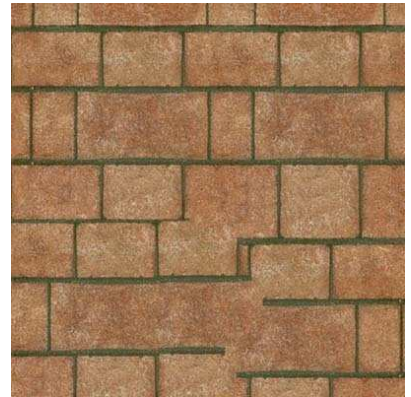

GC $(27.00,0.06, \times, 1.1,0.32)$

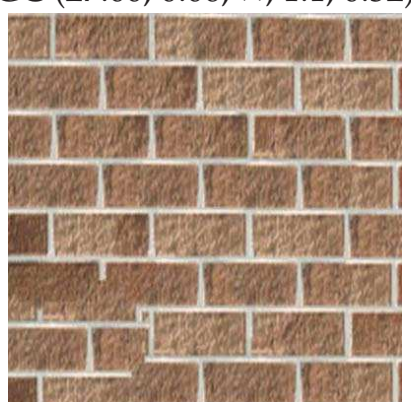

GC $(1.17,0.08, \times, 12,0.42)$

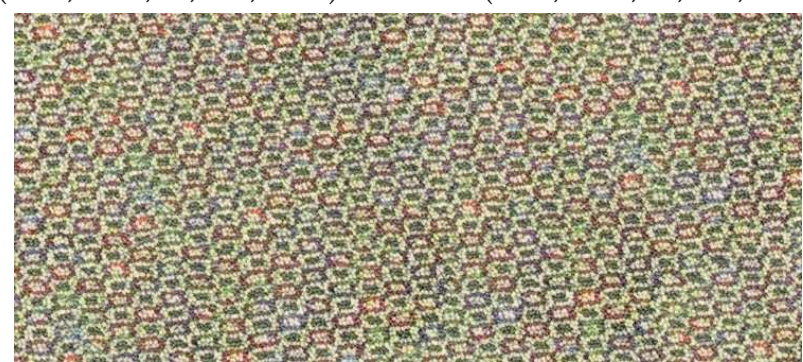

GC $(0.00,0.18, \checkmark, 3.8,0.42)$

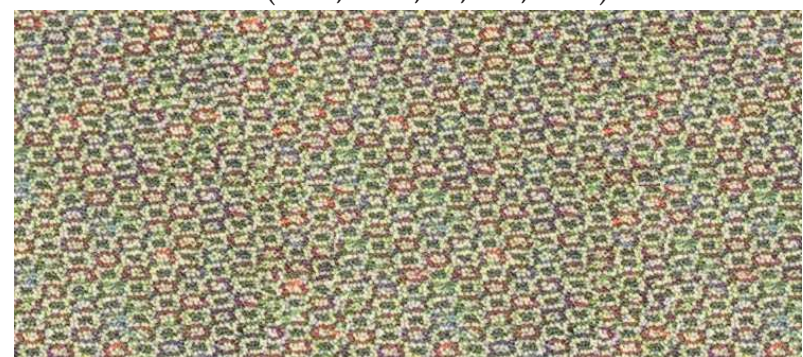

NRTS $(0.00,0.18, \checkmark, 3.7,0.67)$

Figure B.13 


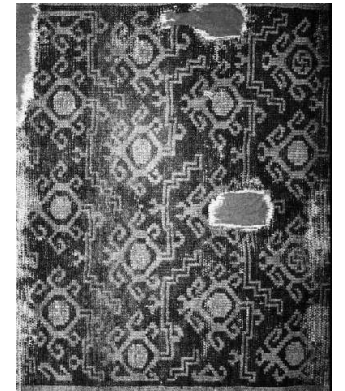

texture $41(2.03,0.17)$

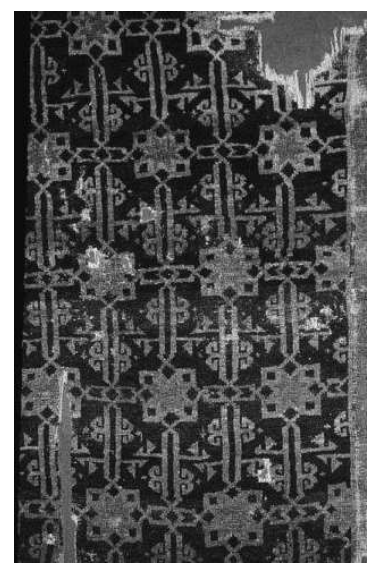

texture $42(0.87,0.11)$

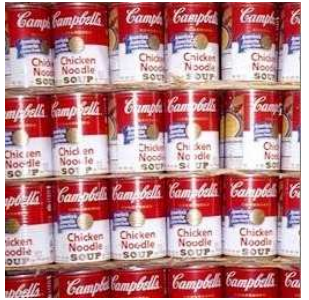

texture $43(0.00,0.15)$

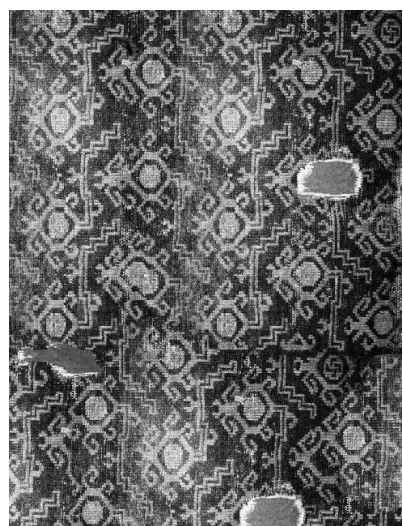

GC $(9.63,0.18, \times 2.3,0.67)$

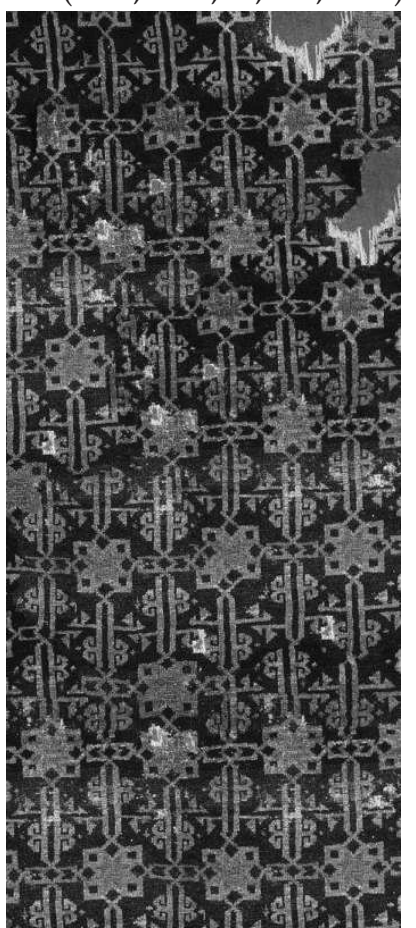

GC $(8.02,0.15, \times, 2.4,0.84)$ F्व

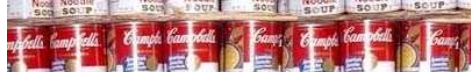

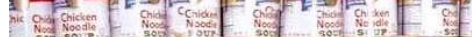

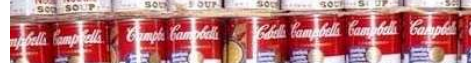
In - 대

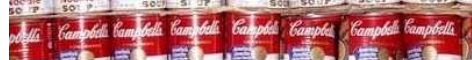

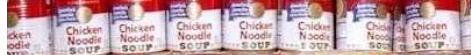

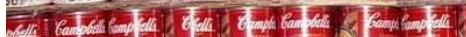

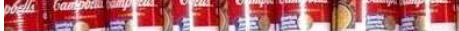

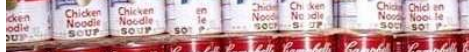

GC $(0.19,0.14, \times, 2.7,1.16)$

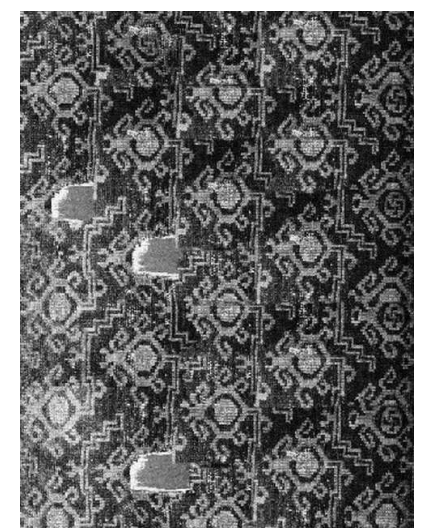

NRTS $(0.96,0.16, \checkmark, 2.7,1.25)$

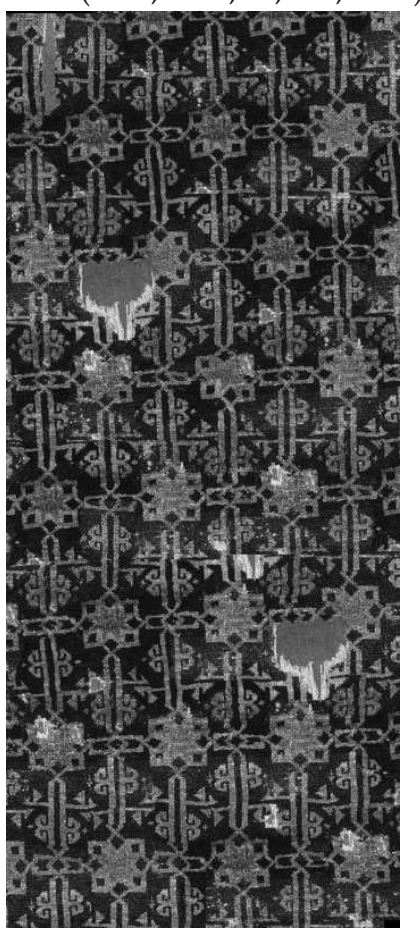

NRTS $(1.17,0.13, \checkmark, 3.3,0.48)$

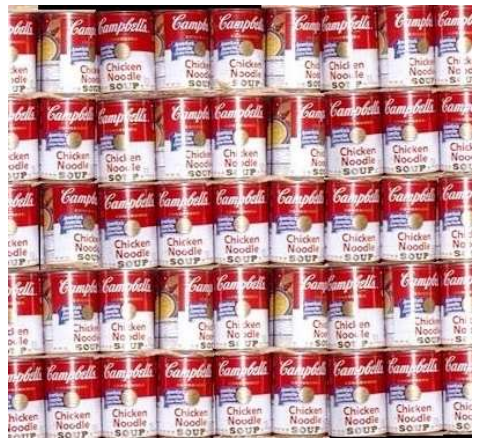

NRTS $(0.20,0.16, \checkmark, 3.4,0.70)$

Figure B.14 


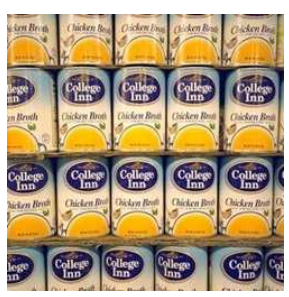

(a) texture 14

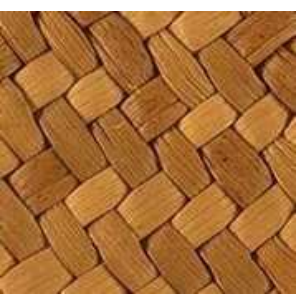

(c) texture 16

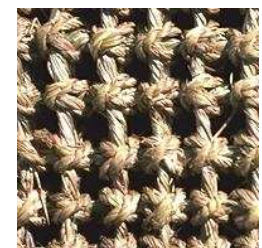

(e) texture 20

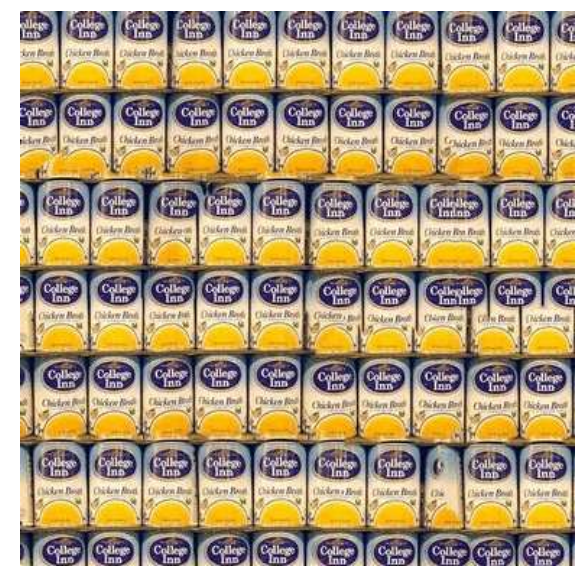

(b) synthesis result

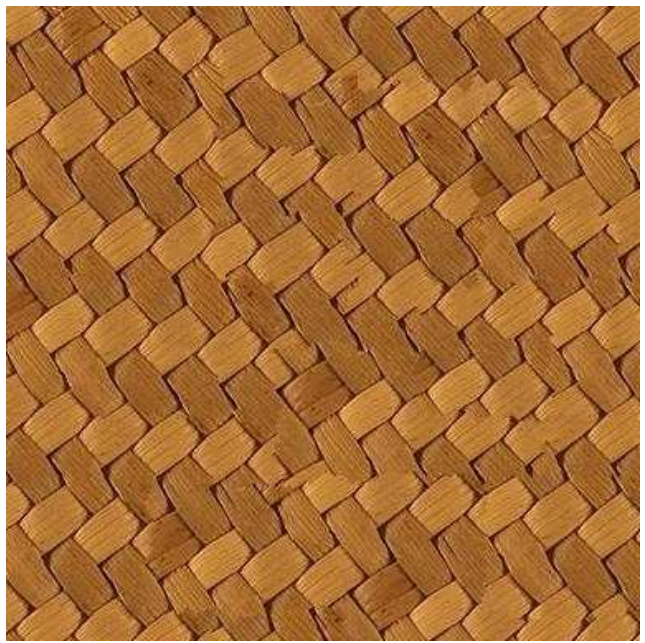

(d) synthesis result

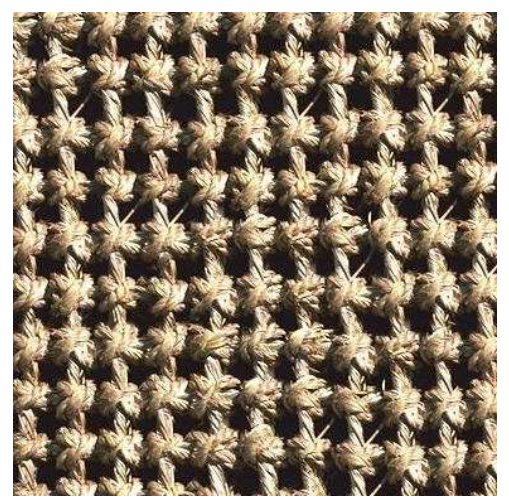

(f) synthesis result

Figure B.15. Texture synthesis results of image quilting [Efros and Freeman, 2001]. 


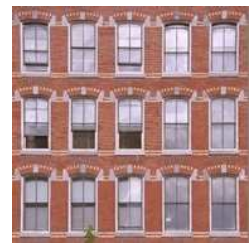

(a) texture 25

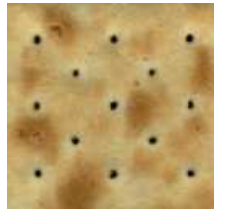

(c) texture 33

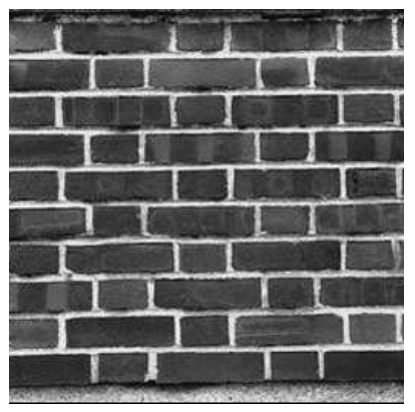

(e) texture 34

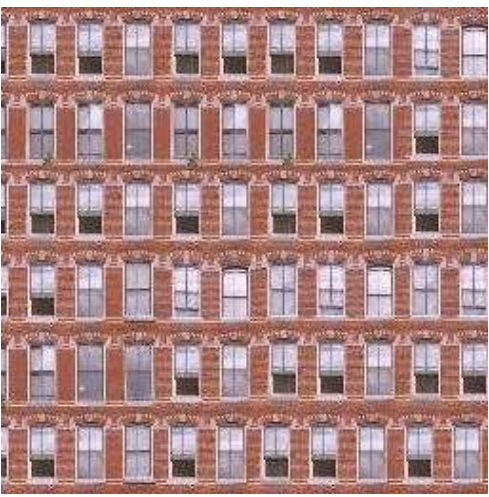

(b) synthesis result

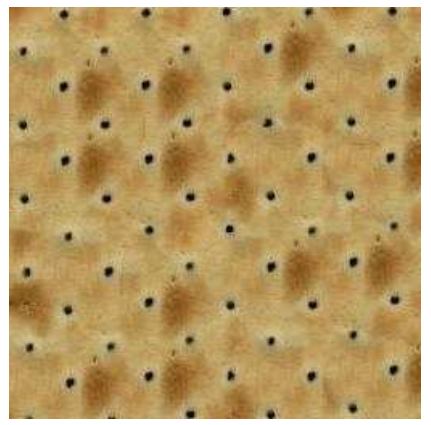

(d) synthesis result

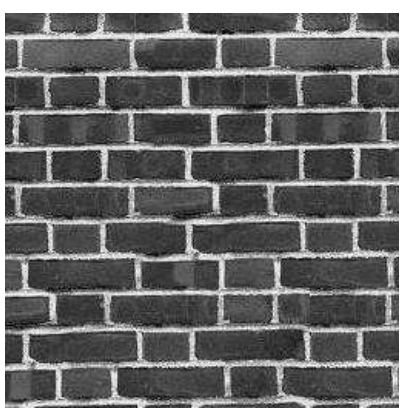

(f) synthesis result

Figure B.16. Texture synthesis results of image quilting [Efros and Freeman, 2001]. 
Document Log:

Manuscript Version 1-August 23, 2005

Typeset by $\mathcal{A}_{\mathcal{M}} \mathcal{S}$-LATEX - 13 January 2006

WEN-CHIEH LIN

The Robotics Institute, Carnegie Mellon University, 5000 Forbes Ave., Pittsburgh, PA 15213, USA, Tel. : (412) 268-8461

E-mail address: wclinecs. cmu.edu 